\title{
ABSTRACTS OF THE 29th ANNUAL MEETING OF THE ITALIAN SOCIETY OF URO-ONCOLOGY (SIURO)
}

11-13 April, 2019, Bologna, Italy

Royal Hotel Carlton, Via Montebello, 8, 40121 Bologna, Italy

Honorary Chair: Enrico Bollito, Eugenio Brunocilla

Italian Society of Uro-Oncology 


\title{
Italian Society of Uro-Oncology (SIUrO)
}

President: Alberto Lapini, Firenze, Italy

\section{Board}

PRESIDENT: Alberto Lapini

VICE PRESIDENT: Renzo Colombo

PAST PRESIDENT, SECRETARY GENERAL \& TREASURER: Giario N. Conti

\section{Advisors}

Alberto Lapini
Riccardo Valdagni
Sergio Bracarda
Orazio Caffo
Renzo Colombo
Giario N. Conti
Rolando M. D'Angelillo

Luigi F. Da Pozzo

Andera B. Galosi

Carlo Patriarca

Alessandro Volpe

Vittorio Vavassori

Nadia Zaffaroni

\section{Elected Scientific Committee \\ Enrico Bollito, Vincenzo Altieri \\ Congress Scientific Committee}

\author{
Enrico Bollito \\ Eugenio Brunocilla \\ Gaetano Facchini \\ Michelangelo Fiorentino
}
Tommaso Prayer Galetti
Roberto lacovelli
Giuseppe Sanguineti
Vincenzo Scattoni

\begin{abstract}
Scientific Secretariat
Società Italiana di Urologia Oncologica (SIUrO)

Via Dante 17 - 40126 Bologna, Italy

Tel: +39051349224 - Fax: +39051349224

e-mail: congresso@siuro.it - web: www.siuro.it

\section{Organizing Secretariat \\ Overgroup}

Via Pagliari, 4-26100 Cremona, Italy

Tel: +39037223310 - Fax: +390372569605

e-mail: info@overgroup.eu
\end{abstract}




\section{Referees of Abstracts}

E. BOLLITO, Orbassano, Italy

R. BORTOLUS, Aviano, Italy

A. BRUNI, Modena, Italy

A. CONTI, Milan, Italy

G. FACCHINI, Naples, Italy

M. FIORENTINO, Bologna, Italy
C. MACCAGNANO, Como, Italy

G. SANGUINETI, Roma, Italy

V. SCATTONI, Milan, Italy

S. SERNI, Florence, Italy

M. TUCCI, Rome, Italy 


\section{1}

\section{SCHISTOSOMIASIS INFECTION MIMICKING A BLADDER CANCER}

Valerio Olivieri ${ }^{1}$, Valentina Fortunati ${ }^{2}$, Luca Bellei ${ }^{1}$,

Massimo Ollino ${ }^{1}$, Massimo Massarelli ${ }^{1}$,

Sandro Guglielmetti ${ }^{1}$, Emanuele Corongiu ${ }^{3}$,

Flavio Forte ${ }^{3}$ and Scipio Annoscia ${ }^{1}$

${ }^{1}$ Division of Urology, Ivrea Hospital, Ivrea, Italy;

${ }^{2}$ Division of Pathology, Policlinico

Tor Vergata, Rome, Italy;

${ }^{3}$ Department of Urology, Hospital M.G.

Vannini, Rome, Italy

Background: Schistosomiasis is an acute and chronic disease caused by parasitic worms: it is transmitted by contact with water contaminated with parasites. Farmers, fishermen and people using unclean water, especially in endemic areas, can be easily infected. Urogenital, intestinal and hepatic localization are common. Furthermore, it is estimated that at least $92 \%$ of patients requiring treatment for schistosomiasis live in Africa. It is a very uncommon disease in Italy, but immigration, especially from North Africa, is changing its prevalence in our country. We describe a case report of a schistosomiasis infection mimicking a bladder cancer in a young black male coming from Africa. Patients and Methods: A 22-year-old man of black ethnicity from Mali presented to the Urology Department for gross hematuria and irritative urinary symptoms. His past medical history was uneventful but he reported a three-month history of lower urinary tract symptoms as urgency and painful micturition associated with recurrent hematuria. All symptoms worsened in the last four weeks. No familiarity for urolithiasis or urothelial cancer was referred during anamnesis. On physical examination he was apyretic and in a good general status. The abdomen was soft and mildly distended: also, male genital inspection was negative and no abnormalities were found on digital-rectal examination. Blood examinations did not show any signs of anaemia or renal impaired function but a high eosinophilia was reported. Moreover, urinalysis showed 1+ haemoglobin (reference range $=0.0$ ): no bacteria, leucocytes, parasitic ova or adult parasites were identified. Furthermore, an abdominal ultrasound showed normal renal parenchyma without hydroneprosis while multifocal and asymmetric bladder wall thickening was detected. Cystoscopy was subsequently performed. The examination revealed multifocal bladder masses highly suspicious for solid urothelial carcinomas and mainly located at different bladder wall. No urinary cytology was performed, but a transurethral resection of bladder (TURB) was offered to patient. His hospital course was regular and he was discharged the next day in a good condition after bladder catheter removal. Results: Pathological analysis of the surgical specimen put in evidence chronic inflammation associated with high eosinophilia and areas of cystitis cystica and cystitis glandularis. The main lesion located at left bladder wall showed chronic inflammation and cystitis glandularis also developing schistosomiasis oviposition (Figure 1). No urothelial cancer specimen was found at pathological analysis totally excluding bladder carcinoma. Discussion and Conclusion: Bladder schistosomiasis, also known as bilharziasis of the bladder, is a parasitic disease. Five species are described, but Schistosoma haematobium is the one mostly linked to human disease. Travellers, immigrants from Africa and people using unclean water in endemic areas are mostly involved. Larvae may easily penetrate the skin of people exposed to the infected water and frequently travel to lung or liver where they reside until maturation: human bladder can be involved too. It represents a major health problem in developing parts of the world predisposing individuals to squamous cell carcinomas because of urothelium changeing through inflammatory insult. Increasing immigratory flow and the variable ethnicity may improve the risk of this parasitic

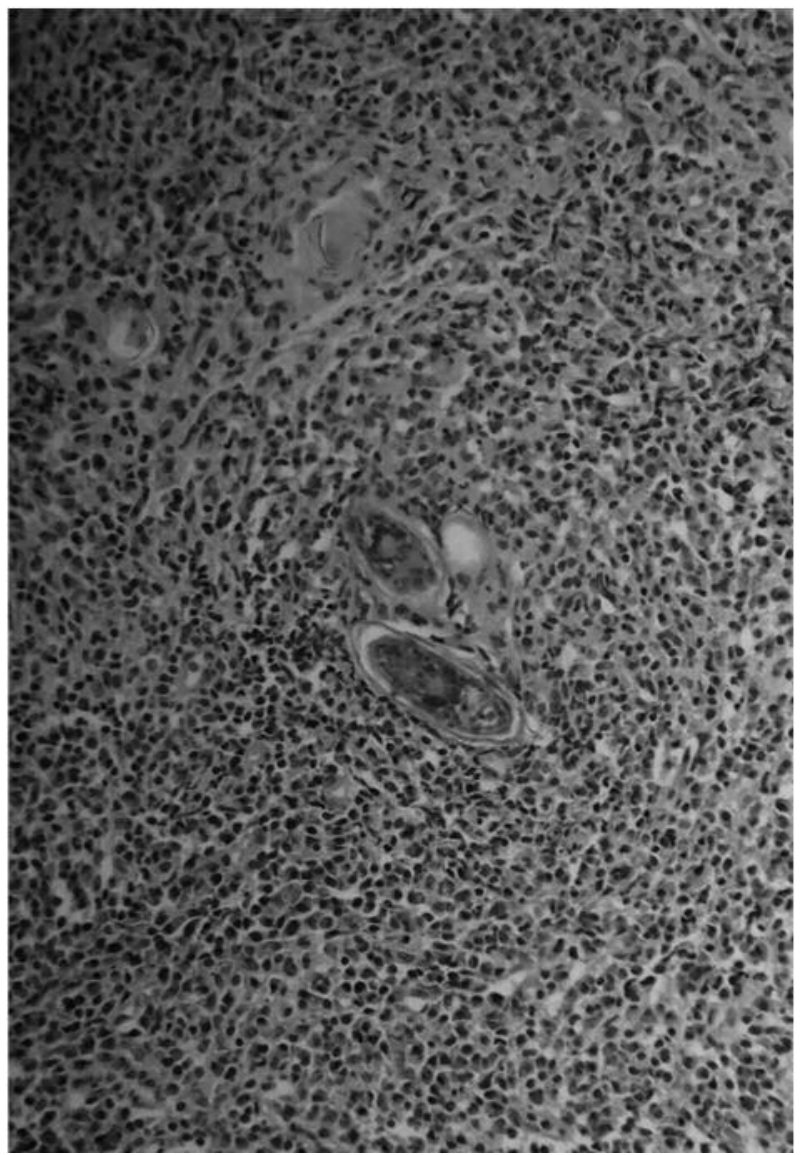

Figure 1. Histopathological section of the left bladder wall main lesion revealing Schistosoma oviposition and tissue inflammation. 
infection also in our country. In the literature few cases of schistosomiasis mimicking bladder cancer are reported and all belong to patients coming from Africa (1). The clinical presentation is similar to bladder cancer often presenting with gross hematuria or bladder thickening during ambulatory ultrasound (2): high eosinophilia is often common but, although indicative, it remains an insufficient screening tool. Screening by serology is easy and reliable, actually representing the gold standard in suspected cases. However, only the histological examinations clarify the presence of infection totally excluding bladder cancer. Clinicians should remember that schistosomiasis is a part of the differential diagnosis of bladder masses especially in patients coming from endemic regions (3).

1 Khaled H: Schistosomiasis and cancer in Egypt: review. J Adv Res 4: 461-466, 2013. PMID: 25685453. DOI: 10.1016/j.jare.2013.06.007

2 Pereira J, Calleja E, Marne C and Borque A: Vesical schistosomiasis with terminal hematuria in sub-Saharan patients. Actas Urol Esp 38: 133-137, 2014. PMID: 24099825. DOI: 10.1016/j.acuro.2013.04.009

3 Zarzour AH, Selim M, Abd-Elsayed AA, Hameed DA and Abdelaziz MA: Muscle invasive bladder cancer in Upper Egypt: the shift in risk factors and tumor characteristics. BMC Cancer 8: 250. PMID: 18759990. DOI: 10.1186/ 1471-2407-8-250

\section{2}

\section{SIGNET RING CELL PROSTATE CARCINOMA: AN AGGRESSIVE HISTOLOGIC VARIANT}

Valerio Olivieri ${ }^{1}$, Valentina Fortunati ${ }^{2}$, Valter Pezzuto ${ }^{3}$, Francesco De Luca ${ }^{4}$, Angela Maurizi ${ }^{4}$, Emanuele Corongiu ${ }^{5}$, Flavio Forte ${ }^{5}$ and Scipio Annoscia ${ }^{1}$

${ }^{1}$ Division of Urology, ASL TO4-Hospital of Ivrea-Chivasso-Ciriè, Ivrea, Italy;

${ }^{2}$ Division of Pathology, Policlinico

Tor Vergata, Rome, Italy;

${ }^{3}$ Division of Pathology, ASL TO4-Hospital

of Ivrea-Chivasso-Ciriè, Ivrea, Italy;

${ }^{4}$ Department of Gynaecological and Urological Sciences,

La Sapienza University of Rome, Policlinico

Umberto I, Rome, Italy;

${ }^{5}$ Division of Urology, Ospedale "Madre

Giuseppina Vannini", Rome, Italy

Background: Signet ring cell prostate cancer (SRCC) is a rare histotype of neoplasm occurring in less than $2.5 \%$ of all prostatic tumors. It is actually considered an aggressive variant of high-grade prostatic adenocarcinoma with poor prognosis and limited life expectancy. Because of its rarity, data in the literature are limited and no recommended treatments have been established to date. We report the case of a signet-ring cell prostate cancer treated with a combination of external beam radiotherapy and total androgen blockade. Patients and Methods: A 78-year-old Caucasian male presented to our facility for PSA raising. On past anamnesis he suffered from myocardial infarction actually on medication with acetilsalicilate; he was also affected by arterial hypertension and mild chronic renal failure with creatinine $1.6 \mathrm{mg} / \mathrm{dl}$. Furthermore, he was on medication with adrenergic a1antagonist for a benign prostatic hyperplasia condition with a post residual void volume of 60 centiliters and International Prostatic Symptoms Score 10. Since many years he has been followed for a PSA raising history with a PSA value $21 \mathrm{ng} / \mathrm{ml}$ in 2016: hence, he previously underwent two prostatic biopsies which resulted both negative for prostate carcinoma. Moreover, no familiarity for prostate cancer was referred by the patient. On blood examination, his last PSA resulted further increased showing a value of $67.5 \mathrm{ng} / \mathrm{ml}$. General and systemic examination was unremarkable: no bone pain, asthenia or weight loss were present. Digito-rectal exploration revealed an enlarged and stony hard prostate expecially on the right lobe, highly suspicious for carcinoma. Thus, the patient underwent a further transperineal prostate biopsy in order to confirm the diagnosis. Results: Pathology demonstrated a Gleason score $10(5+5)$ adenocarcinoma with signet ring cell features affecting $25 \%$ of specimens (Figure 1). The immunophenotypic study showed that the tumor was negative for synaptophysine which frequently results positive in neuroendocrine tumours. Subsequently the patient underwent an abdominopelvic contrast enhanced computed tomography (CT) and total body bone scan in order to exclude metastases. Surprisingly no visceral secundarisms nor bone metastases or enlarged lymph nodes were identified. He elected to undergo a combination of radiotherapy and long term hormonotherapy. Discussion and Conclusion: Signet ring cell cancer is an aggressive histological variant neoplasm. The term "signet ring" describes the histological appearance of a tumor cell characterized by compression of the nucleus into the form of a crescent by a large cytoplasmic vacuole. Although it is primarily found in the stomach and colon, it can less frequently be found also in the pancreas, breast and bladder: primary signet ring cell prostate carcinoma is extremely rare and to date approximately 60 cases have been reported (1). It is actually considered a variant of high-grade prostatic adenocarcinoma and Gleason scoring system should be used while classifying this cancer: regardless of histological classification few data are available in the literature and further investigation is needed. Moreover, it is highly aggressive being usually diagnosed in an advanced stage of disease and with a mean survival of 29 months (2). Gleason score sum of 9 or 10 is typical and it is associated to visceral or bone spread risk. PSA has historically been used as a marker of pathology but some 


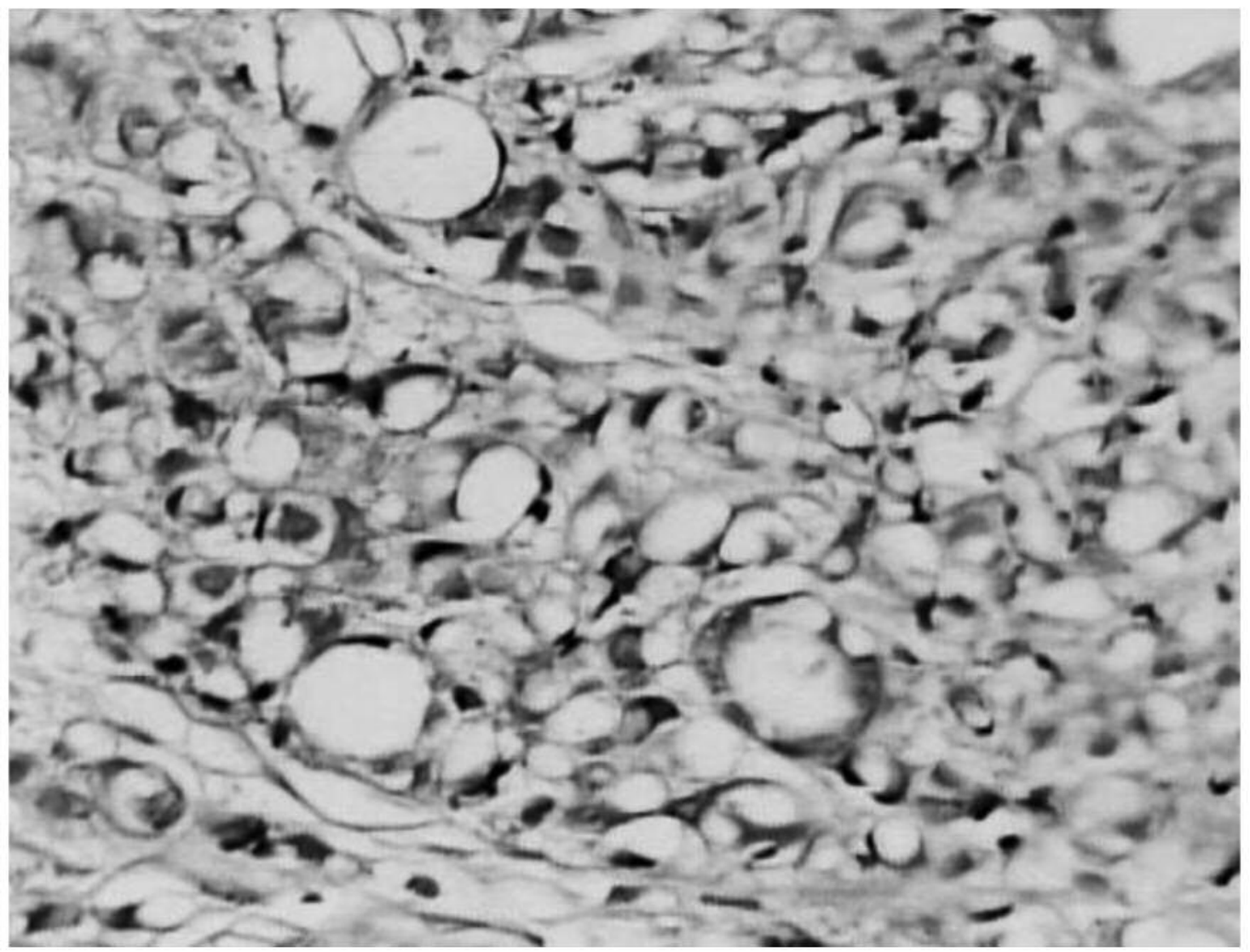

Figure 1. The microscopic finding of prostatic specimen showing the histological variant of signet ring cell prostate carcinoma (original magnification $\times 400$ ).

authors reported how it may be not elevated (3): in our case, serum PSA values and digito-rectal exploration were both higly suspected for neoplasm. The treatment approach for this cancer is similiar to traditional prostatic adenocarcinoma involving different options: radical prostatectomy, radiotherapy, hormonal therapy or variable combinations. Since some authors noted that androgen deprivation therapy alone may be ineffective in some patients affected by this rare histotype, hormonotherapy alone should be avoided while surgery, irradiation or systemic chemotherapy should be first offered. According to the patient's age, performance status and comorbidities, we suggested a combination of radiotherapy and hormonoterapy: precisely he received 35 sessions of external-beam radiation therapy (78 Gy) and total androgens blockade by daily antiandrogens and GnRH-agonist injection every three months. He was initially followed by dosing PSA every month: serum levels of PSA rapidly decreased showing a value of $0.01 \mathrm{ng} / \mathrm{ml}$ at three months. Furthermore, according to histologic variant and the risk of radiological progression despite a biochemical progression, he newly underwent an abdominopelvic CT and bone scan six month later: both of them were negative for metastases. In conclusion, signet ring cell prostate cancer is a rare entity. Approximately 60 cases have been reported to date and limited information is available in the literature. It may be considered as an aggressive high-grade variant of prostatic carcinoma: it usually has a poor prognosis and limited life expectancy. Close follow-up is mandatory. PSA values may be helpful both in making diagnosis and evaluate therapeutic response: many cases without PSA rising have been described. Digito-rectal exploration is frequently suspicious but only histology makes diagnosis. Since many patients are refractory to androgen deprivation therapy, it should be not offered alone while aggressive therapies should be preferred as radical surgery, systemic chemotherapy, irradiation or combined approaches.

$1 \mathrm{Kim}$ SW, Kim W and Sohn DW: Primary ring cell carcinoma of the prostate treated by radical cystoprostatectomy and chemoradiotherapy. Can Urol Assoc J 10(5-6): E204-206, 2016. PMID: 27790307. DOI: 10.5489/cuaj.3122

2 Warner JN, Nakamura LY, Pacelli A, Humphreys MR and Catle EP: Primary signet ring cell carcinoma of the prostate. Mayo Clin Proc 85: 1130-1136, 2010. PMID: 21123640. DOI: $10.4065 / \mathrm{mcp} .2010 .0463$ 
3 Yamamoto S, Ito T, Akiyama A, Aizawa T, Miki M and Tachibana M: M1 prostate cancer with a serum level of prostate-specific antigen less than $10 \mathrm{ng} / \mathrm{ml}$. Int J Urol 8: 374-379. PMID: 21123640. DOI: $10.4065 / \mathrm{mcp} .2010 .0463$

\section{STEREOTACTIC BODY RADIOTHERAPY (SBRT) IN OLIGOMETASTATIC PATIENTS WITH CANCER OF THE PROSTATE}

Barbara Bortolato $^{1}$, Francesco Bracco ${ }^{2}$, Daniela Sibio ${ }^{2}$, Claudia Carbonini ${ }^{3}$, Maria Grazia Brambilla ${ }^{3}$ and Mauro Filippo Palazzi ${ }^{2}$

${ }^{1}$ Department of Radiation Oncology, Niguarda Cancer Center, ASST Ospedale Niguarda, Milan, Italy;

${ }^{2}$ Department of Radiation Oncology, ASST

Ospedale Niguarda, Milan, Italy;

${ }^{3}$ Department of Medical Physics, ASST

Ospedale Niguarda, Milan, Italy

Aim: To evaluate the outcome of patients (pts) treated with SBRT with or without androgen deprivation therapy (ADT) for nodal or bone recurrence after primary local treatment with External Beam Radiotherapy (EBRT) or surgery. Patients and Methods: We retrospectively analyzed data of patients treated with SBRT for oligometastases at the Niguarda Cancer Center between 2010 and 2017. All patients showed less than 3 lesions detected with choline PET-CT or MRI. Biochemical response was evaluated with PSA level variation between pre-RT and post-RT. Biochemical failure was defined as a PSA rising beyond pre-RT levels. PET imaging was performed only in case of biochemical failure to differentiate local from systemic failures. Results: Thirty-six pts were treated on 45 metastatic sites: 27 pts $(60 \%)$ on nodal metastases (NM) and 18 pts $(40 \%)$ on bone metastases (BM). Median age was 76 years. Median PSA before SBRT was $2.48 \mathrm{ng} / \mathrm{ml}$. Initial NCCN class risk was intermediate in 7 pts (19\%) and high in $29(81 \%)$. Androgen deprivation was added in 8 pts (22\%) before SBRT, 18 pts $(50 \%)$ were already receiving hormonal therapy and ten pts $(28 \%)$ were without systemic treatment. Median dose was $30 \mathrm{~Gy} / 3-5$ fractions for BM and 36 Gy/3-4 fractions for NM, lower doses being delivered in previously irradiated volumes. All treatments were image guided (IGRT). Median follow-up was 44 months (range $=4$ 86 months), with two pts (5\%) lost to follow-up. No significant acute or late toxicity was reported. A complete PET response was observed in 39 treated sites $(87 \%)$, while in-field progression or no response occurred at 6 sites (13\%). Twenty pts $(51 \%)$ showed biochemical progression of disease, confirmed by PET imaging in all cases. Median time to biochemical progression was 12 months (range $=3-85$ months). Three pts (8\%) died: two (5\%) for systemic disease progression and one (3\%) for other causes. Conclusion: SBRT for oligometastatic disease due to prostate cancer is a safe treatment modality associated with a high local control rate ( $88 \%$ in our series). Further data are needed to identify patients who could benefit most from this treatment.

\section{4 PURPLE BAG URINE SYNDROME: AN UNCOMMON HUE}

Valerio Olivieri $^{1}$, Valentina Fortunati ${ }^{2}$, Massimo Ollino ${ }^{1}$, Massimo Massarelli ${ }^{1}$, Luca Bellei ${ }^{1}$, Sandro Guglielmetti ${ }^{1}$, Emanuele Corongiu ${ }^{3}$, Flavio Forte $^{3}$ and Scipio Annoscia ${ }^{1}$

${ }^{1}$ Division of Urology, ASL TO4 - Hospital

of Ivrea-Chivasso-Ciriè, Ivrea, Italy;

${ }^{2}$ Division of Pathology, Policlinico

Tor Vergata, Rome, Italy;

${ }^{3}$ Division of Urology, Ospedale "Madre

Giuseppina Vannini", Rome, Italy

Background: Purple urine bag syndrome (PUBS) is an uncommon clinical condition in Urology frequently linked to catheter associated infections (1). It is characterized by a bright purple discoloration of the urine usually affecting patients with limited mobility, long-term catheter and multiple comorbidities. Although considered as a benign phenomenon, it is often associated with increasing anxiety in patient, families and clinicians due to the uncommon color of the urine which is caused by specific bacteria. There are debates regarding how aggressively to treat this condition, but no official guidelines are actually available: reassurance of the patient, early antibiotic regimen and changing of chateter are suggested. We report the case of a palliative patient presenting with this rare condition. Case Report: A 80-year-old caucasian female with a past history of breast and renal cancer was hospitalized in an Oncology facility for a strong back pain. On anamnesis she was affected by ageassociated comorbidities such as osteoporosis, diabetes, arterial hypertension and chronic obstructive pulmonary disease; she also underwent a left radical nephrectomy for oncocytoma. Moreover, two years earlier she underwent a right quadrantectomy with extended lymph nodes dissection due to a breast cancer: histological report revealed an invasive ductal carcinoma pT2 pN0 cM0 Ki67 28\% Erb $>95 \%$ and she underwent adjuvant radiotherapy and chemotherapy with tamoxifen. She reported always good conditions so disappointed the last oncological follow up: she recently developed a back pain associated to legs hyposthenia, highly suspected for vertebral fracture. According to her past anamnesis total body contrast enhanced computerized tomography was performed. The 
examination showed multiple vertebral column metastases and pain was automatically referred to bone pathologic fracture. During hospitalization she received best supportive care and an in-dewelling catheter in order to guarantee immobility leaving her in bed: a progressive purple discolouration of urine was observed during the following days (Figure 1). Results: Urinalysis, culture and blood examination were performed. Blood examination was negative showing normal values of WBC, PCR and creatinine. Urine cultures yielded Pseudomonas aeruginosa as the key pathogen and growth was greater than $10^{5} / \mathrm{ml}$ : moreover, urinalysis revealed an alkaline $\mathrm{pH}$ of 7.5 . Since purple urine bag syndrome may be linked to renal disease, an abdominal ultrasound was performed too: both the kidneys were normal and no stones or hydronephrosis were described. Patient received empirical antibiotic therapy with gentamicin $80 \mathrm{mg}$ intravenous injection 12 hourly and levofloxacin $500 \mathrm{mg}$ daily according to antibiotic sensitivity test of the urine cultures. Furthermore D-mannose and methionine was given in order to correct alkaline ph and speed up the resolution of infection: ten days later urine turned again into normal colour, but therapy had fifteen days of duration. Discussion and Conclusion: Purple urine bag syndrome (PUBS) is a clinical condition that occurs in the presence of urinary catheters and co-existent infection. Risk factors include female gender, chronic catheterizarion and renal disease: moreover, chronic debilitating conditions such as neoplasms or systemic illness may improve the incidence and worse the prognosis. Clinically it is characterized by a purple discolouration of urine (Figure 2) which is usually linked to anxiety in patients and clinicians. The colour is caused as a result of specific bacteria producing sulphatase and phosphatase: this leads tryptophan metabolism to produce indigo and indirubin pigments, a mixture of which becomes purple in alkaline urine (2). Escherichia coli, Pseudomonas aeruginosa, Klebsiella and Proteus are frequently involved (1); alkaline urine is near always reported. Blood examination, urinalysis and culture are mandatory: abdominal ultrasound may be useful in order to exclude hydronephrosis or urinay tract stones. Regarding its management, there are debates on how aggressively to treat and to date no guidelines are available: early antibiotic therapy and changing of catheter are highly suggested. Although considered as a benign condition with a good prognosis, some authors report how it may develop as a complication of urinary tract infection (3): hence, it might be aggressively treated due to the risk of mortality in consideration of patient's background. In our case, patient has been treated with a combination of levofloxacin and gentamicin, methionine, D-mannose and hydration until the purple colour has normalized. In conclusion, purple urine bag syndrome is a rare condition occurring in debilitating patients with long term catheterization and multiple

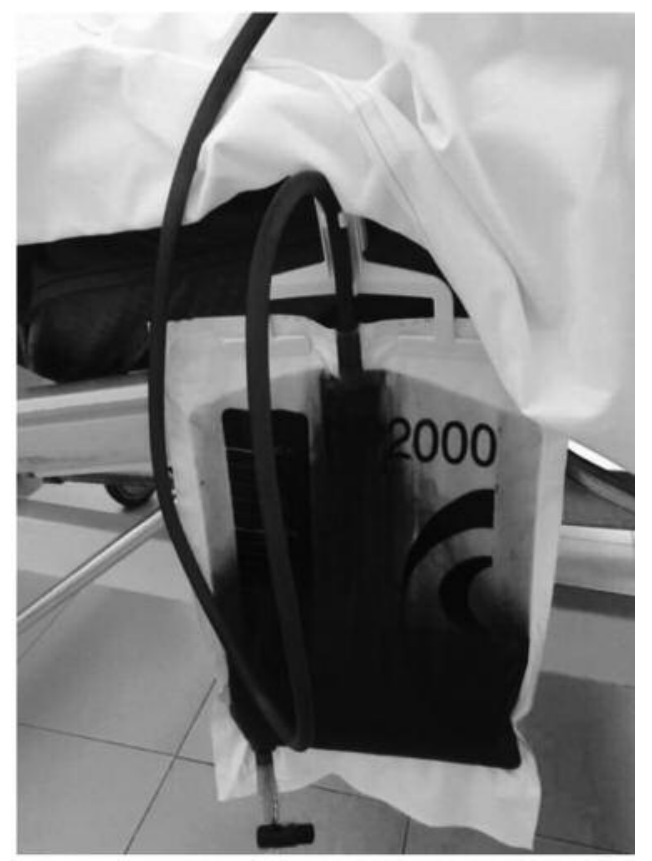

Figure 1. The characteristic purple discolouration of urine observed in PUBS.

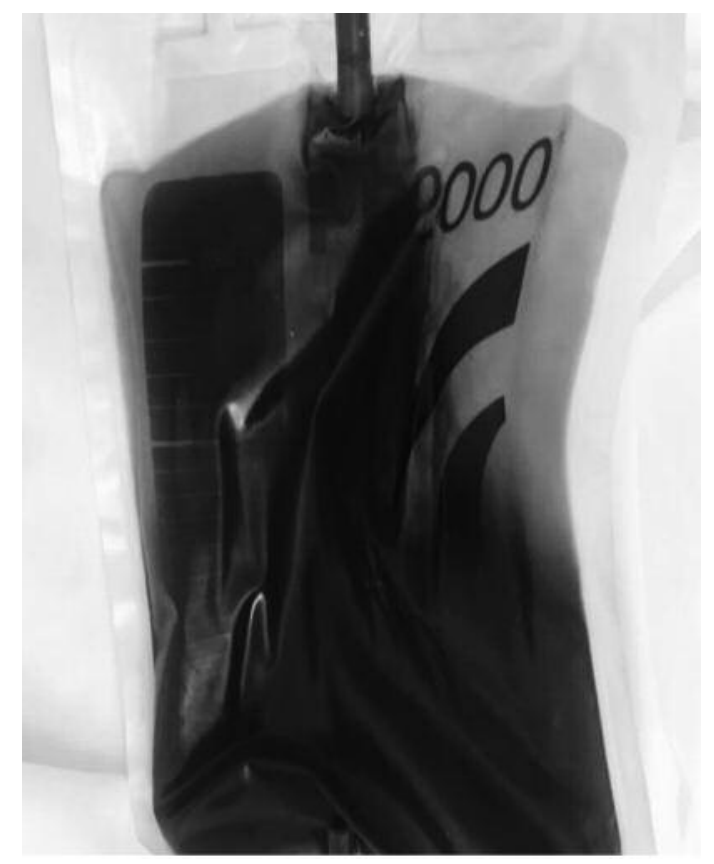

Figure 2. Purple urine as a result of indigo and indirubin pigments mix.

comorbidities. Actually, there are no specific guidelines on how exactly to manage this condition but in general it might be treated aggressively since patient's background may 
influence the risk of mortality: changing drainage bag and indwelling long-term chateter, reassuring patient and starting with early antibiotics therapy as soon as possible is mandatory. Empyrical antibiotic regimen may be offered but since some patients present antibiotic-resistant bacteria, it might be adapted to antibiogram. Futhermore, urine acidification, D-mannose and improvement of hydration may be useful as coadjuvant therapies

1 Lee J: Images in clinical medicine. Purple urine. N Engl J Med 357(13): e14, 2007. PMID: 17898093. DOI: 10.1056/ NEJMicm061573

2 Umeki S: Purple urine bag syndrome (PUBS) associated with strong alkaline urine. Kansenshogaku Zasshi 67(12): 1172-1177, 1993. PMID:8294766.

3 Khan F, Chaudhry MA and Qureshi N: Purple urine bag syndrome: an alarming hue? A brief review of the literature. Int J Nephrol 2011: 419213, 2011. PMID: 21977321. DOI: $10.4061 / 2011 / 419213$

\section{NEPHRON-SPARING ROBOT-ASSISTED \\ PARTIAL NEPHRECTOMY (RAPN) AFTER \\ SUPERSELECTIVE EMBOLIZATION WITH \\ ONYX OF THE RENAL TUMOR: DESCRIPTION \\ OF THE CLAMPLESS TECHNIQUE \\ IN OUR INITIAL EXPERIENCE}

$\underline{\text { Nicolo Fiorello }}^{1}$, Giovanni Luca Giliberto ${ }^{1}$,

Quaretti Pietro $^{2}$ and Lorenzo Paolo Moramarco ${ }^{2}$

${ }^{1}$ Division of Urology, IRCSS Policlinic

Hospital S. Matteo, Pavia, Italy;

${ }^{2}$ Division of Interventional Radiology, IRCSS

Policlinic Hospital S. Matteo, Pavia, Italy

Introduction: Robot-assisted partial nephrectomy (RAPN) is considered the gold standard to treat localized renal tumors, through the use of the PADUA and RENAL score to evaluate and compare the correlations between preoperative and the anatomical aspects. Patients and Methods: In our team we have performed RAPN after superselective embolization with Onyx of the renal tumors with the clampless technique, obtaining a shorter intraoperative resection time of tumor, no time of ischaemia, and reduction of bleeding and intraoperative complicances. This study describes this new approach on 3 patients. The first patient is a 46-year-old woman, with a tumor of $6.2 \mathrm{~cm}$, entirely exophytic, localized on the lower pole of the left kidney with a renal score 5a. Figures 1, 2 and 3 show imaging TC and angiography of renal tumor. The second patient is a 64-year-old woman, with a tumor of $4.1 \mathrm{~cm}$, partially exophytic, localized on the superior pole of the right kidney and a renal score $6 \mathrm{a}$. The

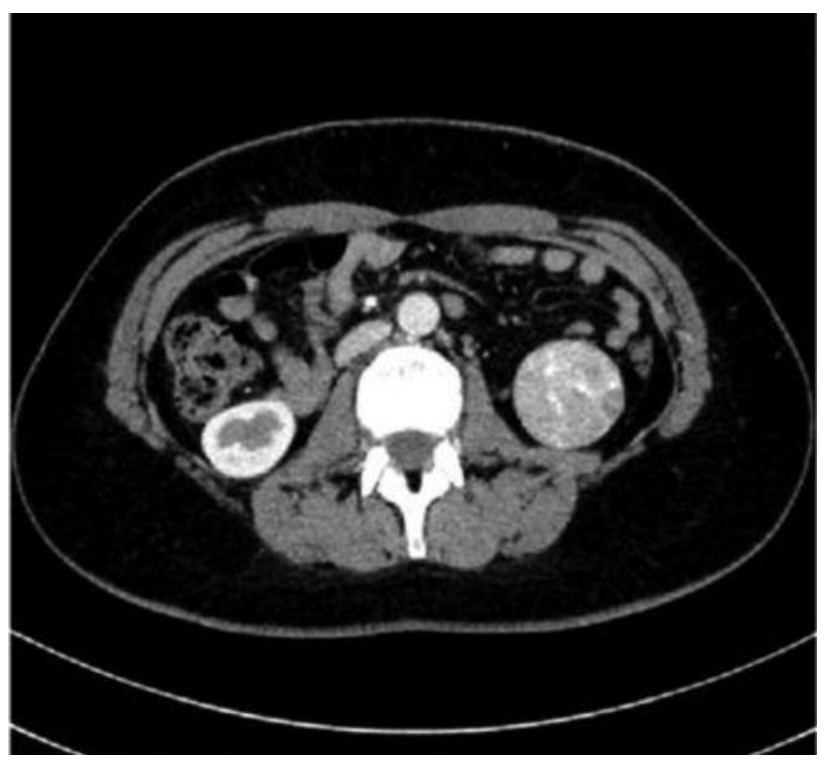

Figure 1. Axial CT of renal tumor of patient no. 1.

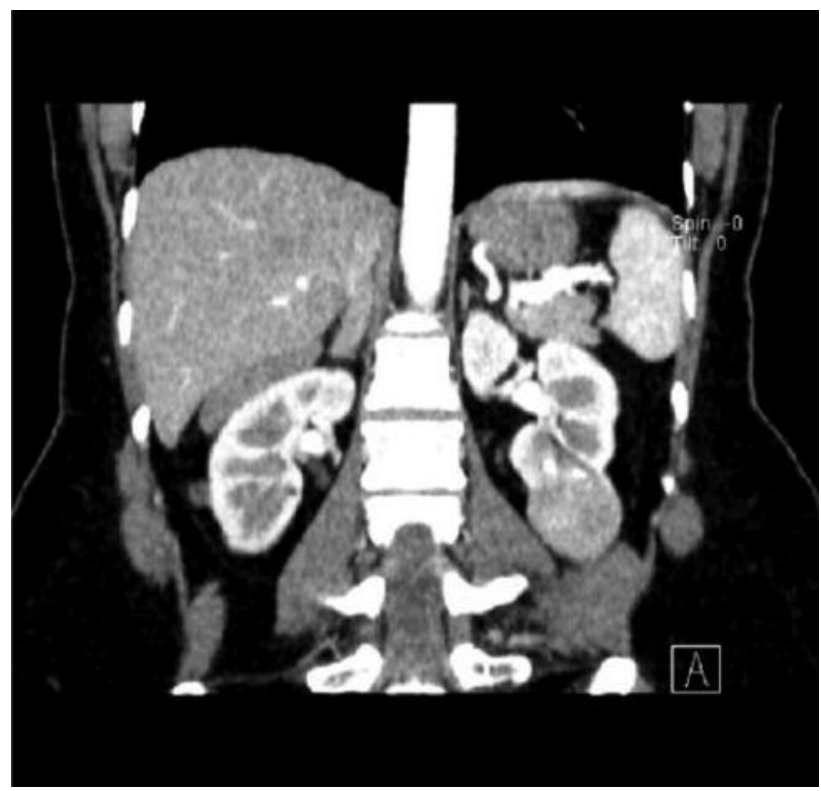

Figure 2. Coronal CT of renal tumor of patient no. 1.

third patient is a 55-year-old man, with a tumor of $4.8 \mathrm{~cm}$, almost entirely exophytic and near to collecting system ( 5 $\mathrm{mm}$ ), of the superior pole of the right kidney and a renal score 6a. Results: In all three patients, the time of hospitalization is reduced, with regular post-operative course and preserved renal functional at 6 months after surgery. Conclusion: Identifying the vascular pedicle in RAPN can 


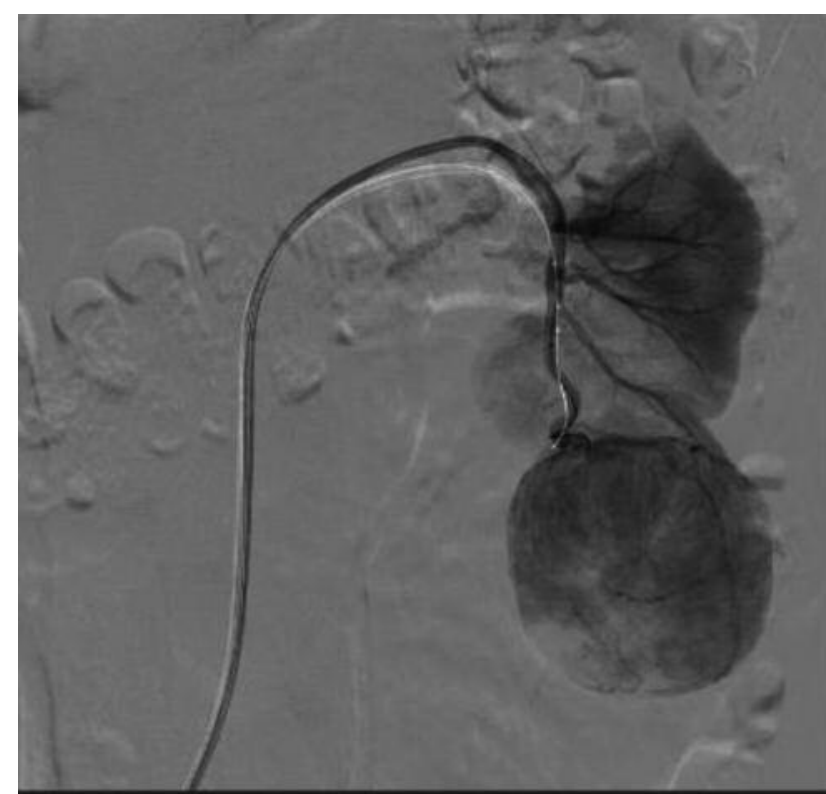

Figure 3. Selective angiography of renal tumor of patient no. 1.

be hard, increasing the risks of intraoperative bleeding and increased surgical resection times. The superselective embolization with Onyx can help the urologist to perform the resection of tumor, with less intraoperative bleeding, shorter resection tumor time.

\section{7}

\section{PREDICTORS OF BIOCHEMICAL RECURRENCE IN PATIENTS TREATED WITH PARTIAL AND WHOLE PROSTATE GLAND CRYOABLATION}

Giovanni Silecchia, Luigi Cormio, Ugo Giovanni Falagario, Oscar Selvaggio and Giuseppe Carrieri

Division of Urology, University of Foggia, Foggia, Italy

Background/Aim: Partial and whole gland cryoablation is a valid alternative to radical treatment for a subgroup of patients at low- to intermediate-risk disease with less sideeffects (1). However, lacking long-term data, there is no consensus regarding the best strategies to follow-up patients after the treatment. The aim of this study was to determine which are the predictive variables of biochemical recurrence after this treatment. Materials and Methods: From our institutional database, we identified 128 patients who underwent partial $(56,43 \%)$ and whole gland $(72,57 \%)$ cryoablation for systematic TRUS biopsy proven unilateral or bilateral prostate cancer respectively. Partial and wholegland cryoablation was performed by a free-hand technique under TRUS guidance using an argon/helium gas- based system (Endocare, HealthTonics Inc., Austin, TX, USA), with a double freeze-thaw cycle (2, 3). All patients maintained an ${ }^{18} \mathrm{~F}$ Foley catheter for 1 week and 10 days respectively after partial and whole cryoablation that was discontinued after a voiding trial. Follow up was scheduled with a serum PSA every three months and a mpMRI 6 month after partial gland procedure. Of each patient, we recorded data about age, clinical stage, preoperative PSA, ISUP, prostate density, prostate volume, PSA nadir and cryoprobe number. We analyzed factors associated with biochemical recurrence defined according to the Phoenix criteria as a PSA rising of more than $2 \mathrm{ng} / \mathrm{ml}$ after PSA nadir. Cox regression univariate and multivariate analysis was done to determine the clinical indicators that predict for PSA failure. Kaplan Meier analysis was run to estimates overall progression free survival after partial and whole gland treatment (Figure 1). Results: Patients's baseline characteristics and predictive variables are reported in Table I. Using EAU risk stratification criteria based on D'Amico (4), 49 had low risk disease (ISUP 1 with PSA $<10$ and cT1ccT2a), 31 had Intermediate Risk disease (ISUP 2 or PSA 10$<20$ or cT2b) and 48 patients had high Risk disease (ISUP $>3$ or PSA $>20$ or cT2c). Biochemical recurrence rate for partial and whole gland treatment was $9(5 / 56)$ and $13 \%(9 / 72)$ of patients respectively until to 55 months of follow-up. Local recurrence was defined as prostate cancer in treated lobe or

Table I. Demographics characteristics.

\begin{tabular}{lcc}
\hline Variable & $\begin{array}{c}\text { Partial gland } \\
\text { No. of } \\
\text { patients=56 }\end{array}$ & $\begin{array}{c}\text { Whole gland } \\
\text { No. of } \\
\text { patients=72 }\end{array}$ \\
\hline Age & $78(71,80)$ & $77(73,79)$ \\
Clinical stage, N (\%) & $38(68 \%)$ & $46(64 \%)$ \\
1 & $8(14 \%)$ & $0(0 \%)$ \\
2 & $8(14 \%)$ & $1(1 \%)$ \\
3 & $2(4 \%)$ & $24(33 \%)$ \\
4 & $0(0 \%)$ & $1(1 \%)$ \\
5 & $6.11(4.70,8.19)$ & $5.82(4.84,9.20)$ \\
PSA & & \\
ISUP, N (\%) & $33(59 \%)$ & $16(22 \%)$ \\
1 & $13(23 \%)$ & $18(25 \%)$ \\
2 & $4(7 \%)$ & $14(19 \%)$ \\
3 & $6(11 \%)$ & $20(28 \%)$ \\
4 & $0(0 \%)$ & $4(6 \%)$ \\
5 & $0.13(0.10,0.20)$ & $0.15(0.10,0.25)$ \\
PSA density & $48.00(37.50,62.75)$ & $42.00(30.00,56.00)$ \\
Prostate volume & $1.12(0.50,2.72)$ & $0.20(0.09,0.65)$ \\
PSA nadir & $4.00(3.00,4.00)$ & $6.00(6.00,6.00)$ \\
$N^{\circ}$ AGHI & $51(91 \%)$ & $62(87 \%)$ \\
Recurrence, N $(\%)$ & $5(9 \%)$ & $9(13 \%)$ \\
0 & & \\
1 & & \\
\hline
\end{tabular}


Table II. Univariate and multivariate analysis.

\begin{tabular}{|c|c|c|c|c|c|c|c|c|}
\hline & \multicolumn{4}{|c|}{ Univariate } & \multicolumn{4}{|c|}{ Multivariate } \\
\hline & Haz. ref. & \multicolumn{2}{|c|}{$95 \%$ C.I. } & $\mathrm{P}>|\mathrm{z}|$ & Haz ref. & \multicolumn{2}{|c|}{$95 \%$ C.I. } & $\mathrm{P}>|\mathrm{z}|$ \\
\hline \multicolumn{9}{|l|}{ ISUP } \\
\hline \multicolumn{9}{|l|}{1} \\
\hline 2 & 3.023 & 0.273 & 33.362 & 0.366 & 5.975 & 0.457 & 78.110 & 0.173 \\
\hline 3 & 14.807 & 1.726 & 127.013 & 0.014 & 26.943 & 2.178 & 333.294 & 0.01 \\
\hline 4 & 7.346 & 0.856 & 63.019 & 0.069 & 15.922 & 1.240 & 204.396 & 0.034 \\
\hline 5 & 12.201 & 0.755 & 197.028 & 0.078 & 27.506 & 1.262 & 599.151 & 0.035 \\
\hline Pre op PSA & 1.027 & 1.001 & 1.053 & 0.04 & 0.929 & 0.870 & 0.992 & 0.029 \\
\hline PSA nadir & 1.145 & 1.026 & 1.278 & 0.016 & 1.521 & 1.152 & 2.009 & 0.003 \\
\hline Partial gland & ref. & & & & ref. & & & \\
\hline Whole gland & 1.199 & 0.401 & 3.584 & 0.744 & 2.035 & 0.397 & 10.433 & 0.394 \\
\hline
\end{tabular}

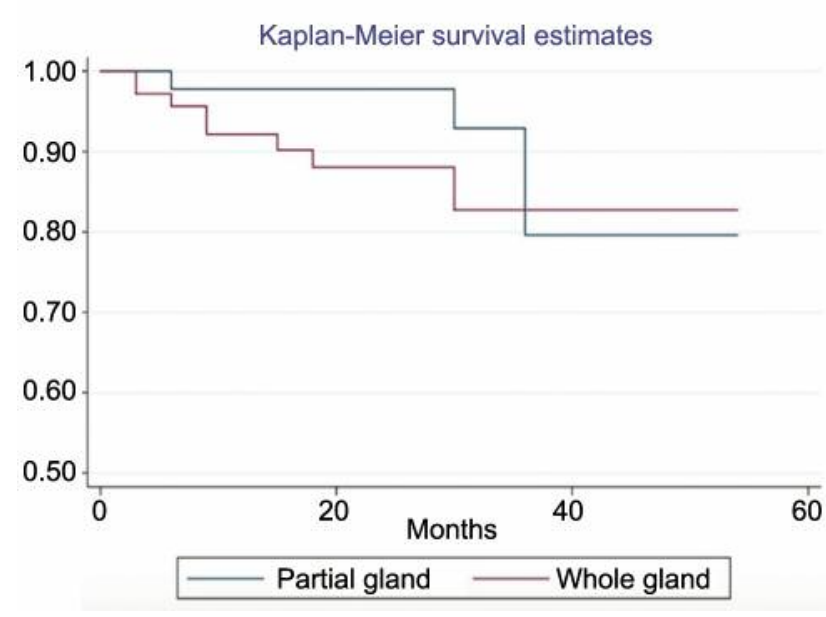

Figure 1. Blue line: biochemical recurrence free survival in partial gland cryoablation; Red line: biochemical recurrence free survival in whole gland cryoablation.

both lobes respectively in partial and whole gland cryoablation; tumor progression was defined as prostate cancer in non-treated lobe in partial gland cryoablation. In the last three years, follow-up is completed with mpMRI in partial treatment. All of them underwent MRI/target biopsy with diagnosis of cancer progression or local recurrence and then underwent to a radical cryoablation of prostate in previous treated partial ablations while to androgen deprivation therapy and re-cryoablation respectively in 7 and 6 patients previously treated whole gland ablation with, actually, undosable PSA. At 55 months of follow-up no patients showed distant metastases. Univariate and multivariate Cox regression analysis showed that preoperative PSA, PSA nadir, ISUP 3-4-5 are associated with a higher risk of biochemical recurrence (Table II). Discussion:
This study is limited by its retrospective design. Patients were selected by TRUS biopsy rather than with MRI and consequent MRI/TRUS fusion biopsy, which could add accuracy to staging and potentially provide better outcomes. However, in the last three years, MRI is part of the followup protocol. So, this new imaging technique may have identified recurrences earlier. Cryoablation is a safe, minimally-invasive procedure that uses cold temperatures delivered via probes through the skin to kill prostate cancer (PCa) cells. Partial and whole gland cryoablation may offer an alternative treatment option to surgery and radiotherapy. We found that patients had good cancer outcomes to 55 months after partial and whole gland cryoablation. Conclusion: In patients suitable for partial and whole gland, on the basis of parameters suggested by EAU guidelines, around 91 and $87 \%$ respectively are biochemical recurrence free to 55 months. We identified higher PSA nadir, preoperative PSA and ISUP particularly grade 3, 4 and 5 as predictive factors of biochemical recurrence after cryoblation therapy for clinically localized prostate cancer.

1 Cornford P, Bellmunt J, Bolla M, Briers E, De Santis M, Gross T, Henry AM, Joniau S, Lam TB, Mason MD, van der Poel HG, van der Kwast $\mathrm{TH}$, Rouvière $\mathrm{O}$, Wiegel $\mathrm{T}$ and Mottet N. EAU-ESTRO-SIOG Guidelines on prostate cancer. Part II: treatment of relapsing, metastatic, and castration-resistant prostate cancer. Eur Urol 71: 630-642, 2017. PMID: 27591931. DOI:10.1016/j.eururo.2016.08.002 2 de Castro Abreu AL, Bahn D, Leslie S, Shoji S, Silverman P, Desai MM, Gill IS and Ukimura O: Salvage focal and salvage total cryoablation for locally recurrent prostate cancer after primary radiation therapy. BJU Int 112: 298307, 2013. PMID: 23826840. DOI: 10.1111/bju.12151

3 Bahn D, de Castro Abreu AL, Gill IS, Hung AJ, Silverman P, Gross ME, Lieskovsky $\mathrm{G}$ and Ukimura O: Focal 
cryotherapy for clinically unilateral, low-intermediate risk prostate cancer in 73 men with a median follow-up of 3.7 years. Eur Urol 62: 55-63, 2012. PMID:22445223. DOI:10.1016/j.eururo.2012.03.006

4 D'Amico AV, Moul J, Carroll PR, Sun L, Lubeck D and Chen MH: Cancer-specific mortality after surgery or radiation for patients with clinically localized prostate cancer managed during the prostate-specific antigen era. J Clin Oncol 21: 2163-2172. PMID:12775742. DOI: 10.1200/JCO.2003.01.075

\section{8}

\section{FUSION BIOPSY FOR DETECTING PROSTATE CANCER. IS IT REALLY BETTER?}

Cristina Falavolti ${ }^{1}$, Ervin Shehu ${ }^{1}$, Riccardo Giovannone ${ }^{1}$, Pouria Alijani ${ }^{1}$, Roberto Giulianelli ${ }^{2}$, Tommasangelo Petitti ${ }^{3}$ and Maurizio Buscarini ${ }^{4}$

${ }^{1}$ Division of Urology, Villa Betania

Hospital of Rome, Rome, Italy;

${ }^{2}$ Division of Urology, Nuova Villa Claudia

Clinic of Rome, Rome, Italy;

${ }^{3}$ Division of Hygiene and Public Health, University

Campus Biomedico of Rome, Rome, Italy;

${ }^{4}$ Division of Urology, University Campus

Biomedico of Rome, Rome, Italy

Introduction: European guidelines are changing and strongly recommend to include to the ultrasound-guided biopsy, systematic biopsies and targeting of any mpMRI lesions. A standardized template is not yet available. The aim of this study was to demonstrate if targeted magnetic resonance/ultrasound fusion prostate biopsy is really more accurate than the traditional procedure and which parameters significantly affect it. Materials and Methods: We prospectively enrolled 56 patients who underwent fusion biopsy at our Institution from January 2018 to May 2018. All biopsies were performed by the same operator (C.F.) with the same instrument, KOELIS ${ }^{\circledR}$. Procedures consisted of collecting samples obtained by targeted magnetic resonance/ultrasound fusion prostate biopsy plus random samples obtained from both lobes (right and left) in a traditional modality (ultrasound-guided only). In 24 cases our procedure was a repeat biopsy after previous ultrasound-guided biopsy resulted negative but with still a PSA value increasing. We collected data regarding pre-biopsy PSA value and size of the prostate. For each lesion detected at the mpMRI, we analyzed the location and the size. We counted the number of cores sampled during the biopsy for each site and related the histological report. A statistical analysis was performed using logistic regression to verify the modification of positivity risk in specific conditions. We used also the $t$-test to verify the differences between groups. Results: Patients mean age was 64 years (range $=48-80$ years). In our series transitional zone of the prostate was less affected by cancer than peripheral zone $(p=0.066)$. The more the gland was big the less the cancer was easily detected. Previous biopsies did not affect the probability to find a prostate cancer during the fusion biopsy. Number of cores and randomized samples did not change the probability to find prostate cancer (Table I). Thus, the probability to find prostate cancer in randomized cores was so low that the ultrasound-guided biopsies in both right and left lobes did not improve cancer detection in the same patient.

Table I. Parameters that significantly affect targeted magnetic resonancelultrasound fusion prostate biopsy.

\begin{tabular}{lcr}
\hline Parameters & $\begin{array}{c}\text { Cancer positivity } \\
\text { Odd Ratio }\end{array}$ & $p$-Value \\
\hline Prostate dimension (mm) & 0.09 & $<0.05$ \\
Randomized cores & 1.13 & 0.27 \\
Previous biopsy & 0.95 & 0.89 \\
\hline
\end{tabular}

Discussion: Fusion prostate biopsy is still considered a novel procedure, but is spreading all over the world. Our data suggest that targeted cores have significantly higher probability to detect prostate cancer than randomized samples, prostate dimension is significantly related with the easiness to detect the cancer, and a previous negative traditional prostate biopsy does not change the probability to detect a significant prostate cancer. More data are needed to achieve stronger results to completely avoid the randomized biopsies and significantly reduce the cores number of each procedure.

9

PROSPECTIVE OBSERVATIONAL STUDY ON REAL-LIFE LABORATORY AND CLINICAL INVESTIGATIONS IN PATIENTS TREATED WITH GONADOTROPIN-RELEASING HORMONE ANALOGUES

Cristina Scalici Gesolfo ${ }^{1}$, Alfredo Colombo ${ }^{2}$, Rosa Maria Valerio ${ }^{3}$, Giuseppe Ferrera ${ }^{4}$, Michele Ruoppolo ${ }^{5}$, Emanuele Caldarera ${ }^{1}$, Michele Barbera $^{6}$, Danilo Di Trapani ${ }^{7}$, Simona Grutta ${ }^{3}$, Chiara Sanfilippo ${ }^{8}$, Ivan Fazio ${ }^{9}$, Vincenzo Serretta ${ }^{3}$ and Members of GSTU Foundation ${ }^{10}$

${ }^{1}$ Department of Urology, Paolo

Borselino Hospital, Marsala, Italy;

${ }^{2}$ Medical Oncology Unit, Macchiarella Clinic,

Palermo, Italy;

${ }^{3}$ Section of Medical Oncology, Department of Surgical, Oncological and Oral Sciences, University of Palermo, Palermo, Italy; 
${ }^{4}$ Radiotherapy Unit, ARNAS Civico

Hospital, Palermo, Italy;

${ }^{5}$ Department of Urology, San Giovanni

di Dio Hospital, Agrigento, Italy;

${ }^{6}$ Department of Urology, Giovanni

Paolo II Hospital, Sciacca, Italy;

${ }^{7}$ Department of Urology, Buccheri

La Ferla Hospital, Palermo, Italy;

${ }^{8}$ Statistics, GSTU Foundation, Palermo, Italy;

${ }^{9}$ Radiotherapy Unit, Macchiarella Clinic, Palermo, Italy;

${ }^{10}$ Other Members, GSTU Foundation, Palermo, Italy

Introduction: Androgen-deprivation therapy (ADT) with gonadotropin-releasing hormone $(\mathrm{GnRH})$ agonists or antagonists is the mainstay of treatment of locally advanced and metastatic prostate cancer (PCa). Plasmatic testosterone (T) castrate level lower than $50 \mathrm{ng} / \mathrm{Dl}$, or better lower than $20 \mathrm{ng} / \mathrm{Dl}$, must be reached to obtain the better results. ADT has several side-effects including sexual dysfunction, bone demineralization, cardiovascular disease and metabolic complications. Minimal baseline clinical and laboratory examinations are mandatory before starting ADT and during follow-up to verify $\mathrm{T}$ levels and evaluate patients' comorbidities and health status. The aim of this study was to investigate the management of patients undergoing ADT in everyday clinical practice in our region. Materials and Methods: A prospective observational study was planned for patients affected by advanced or metastatic PCa and undergoing first-line ADT. Patients' characteristics and investigations performed at baseline and at follow-up should be given within one month. The examinations were done at physicians' discretion according to their everyday clinical practice and reported in a dedicated database. Previous hormone therapy, untreated localized prostate cancer and age over 85 years where the main exclusion criteria. Results: From December 2017 to September 2018, 104 patients were entered. Twenty-seven (26\%) progressed after radical prostatectomy or radiotherapy, $16(15.3 \%)$ had a locally advanced tumor and $75(72.1 \%)$ a metastatic tumor. Therapy was prescribed by oncologists, urologists and radiotherapists in $22(21.2 \%), 17(16.3 \%)$ and $65(62.5 \%)$ patients, respectively. Median age was 77 years. LHRH agonist and antagonist was administered in $49(47 \%)$ and 55 $(53 \%)$ patients, respectively. At baseline, plasmatic testosterone $(\mathrm{T})$, alcaline phosphatase (Palk), were obtained in only $36(35 \%)$ and $49(47 \%)$ patients respectively. At 1 and 3 months, data did not change significantly since $\mathrm{T}$ was obtained in $37 \%$ and in $56 \%$ of patients respectively, while Palk at 3 months in $47 \%$ of patients. Baseline PSA, creatinine, haemoglobin, performance status, BMI and ECG with cardiological visit were obtained in $104(100 \%), 74$ (71\%), 93 (89\%), 97 (93\%), 98 (94\%) and $82(79 \%)$ patients respectively. Similar percentages were maintained at 3-month follow-up. Discussion and conclusion: In everyday clinical practice when prescribing first-line ADT for advanced $\mathrm{PCa}$, the physicians pay adequate attention to the patients' general status but are less accurate, both at baseline and within 3 months, in verifying $T$ and Palk giving up to exams of primary relevance to monitor the efficacy of ADT.

The Authors wish to thank the GSTU Foundation for the redaction and statistical support

\section{0}

\section{FACTORS PREDICTING THE DEVELOPMENT OF SYMPTOMATIC LYMPHOCELES AFTER LYMPH NODE DISSECTION IN ROBOT-ASSISTED RADICAL PROSTATECTOMY: MONOCENTRIC EXPERIENCE AND REVIEW OF THE LITERATURE}

Lucio Dell'Atti, Carmine Franzese, Camilla Capretti, Erika Palagonia, Alessio Papaveri, Lorenzo Montesi and Andrea Benedetto Galosi

Institute of Urology, University Hospital "Ospedali Riuniti", Polytechnic University of Marche Region, Ancona, Italy

Introduction: Pelvic lymph node dissection (PLND) is the gold standard for detection of nodal (LN) staging in prostate cancer. Despite the benefits of staging accuracy and its possible therapeutic role in eliminating microscopic metastases, PLND is also associated with several disadvantages, such as increased morbidity, longer operation time, and higher costs $(1,2)$. The most frequent postoperative complications of PLND are lymphoceles, although not all lymphoceles need further surgical treatment and can be managed conservatively. The incidence of lymphoceles is reported between 2 and $9 \%$ of the patients (2). In the literature several factors provoking lymphocele formation have been discussed, including the patient's age, experience of the surgeon, and the extent of PLND (3). In this retrospective single-surgeon study, we wished to analyze predictors of symptomatic lymphoceles (SL) after robot-assisted radical prostatectomy (RARP) in patients affected by localized prostate cancer. Materials and Methods: We retrospectively reviewed the clinical data of a single-surgeon series of 181 consecutive patients affected by prostate cancer and undergoing RARP and PLND between January 2016 and September 2018. The exclusion criteria for study enrollment were: patients with previous treatments for prostate cancer (including surgery, radiotherapy, hormone therapy and/or chemotherapy). The decision to perform PLND was usually based on cancer aggressiveness and in all men with a risk of lymph node metastasis $>5 \%$ based on the updated Briganti nomogram. 
Data were collected, including age, body mass index (BMI), pre- and post-operative PSA, prostate volume, pathological data (Gleason score, stage classification), and complications classified according to the Clavien-Dindo classification. The occurrence of SL was identified in patients undergoing imaging as a result of symptoms suspicious for lymphocele, such as fever, abdominal pain, lower-extremity swelling or hydrocele. The PLND was performed on the external iliac, obturator, internal iliac, and common iliac up to the ureter crossing. In addition, the lymphofatty tissue of the periprostatic area was sent separately to pathology. All PLNDs were performed after radical prostatectomy. Following RARP, one gravity drain was placed monolaterally into the paravesical space. No hemostatic sealants were used for prevention of lymphocele formation. Drains were removed after a mean of 2.4 (2-6) days postoperatively when drainage was less than $70 \mathrm{ml}$ within $24 \mathrm{~h}$. All histological specimens were analyzed internally by our Pathology Department specializing in genitourinary pathology. Cases were not reviewed for this study. Statistical analyses were carried out using Microsoft Excel 2010 platform version $10.1 p<0.05$ was considered statistically significant. Results: RARP was carried out utilizing a transperitoneal approach in all patients. Robotic PLND was performed in 103 (57\%) of patients [46 (25\%) unilaterally and 57 (31\%) bilaterally]. Mean age at surgery was 63.8 (43-83) years and median PSA was 7.5 (1.3-18.5) $\mathrm{ng} / \mathrm{ml}$. The median prostate volume was $42(23-100) \mathrm{ml}$. The median BMI was $26(19-40) \mathrm{kg} / \mathrm{m}^{2}$. The median operation time for RARP with PLND was 255 (220-350) min. The median estimated blood loss from RARP with PLND was $240(20-900) \mathrm{ml}$, and $4(2.2 \%)$ patients received a blood transfusion. Out of the 103 patients, $7(6.8 \%)$ and $29(28.1 \%)$ had LN metastases, unilaterally $v s$. bilaterally respectively. The incidence of $\mathrm{LN}$ metastases was correlated with Gleason score and tumor stage. A total of 83 positive LNs were found, and the median number of positive LNs in patients with LN metastases was 2 (1-11). Overall, a median of 22 LNs (7-53) were removed from all patients. Complications associated with LND occurred in 11 patients $(10.6 \%)$. Clavien Grade 3 complications were observed in $3(2.9 \%)$ patients who underwent percutaneous drainage for SL by an interventional radiologist. SL was found in 5 patients $(4.9 \%)$ and 2 patients were treated conservatively. Of the 5 patients with symptomatic lymphocele, one was diagnosed one month after surgery owing to spiking fever, while the others were diagnosed during their postoperative hospital stay. Lymphedema was observed in 4 patients (3.9\%), which resolved after assumption of diuretics drugs and physical treatment. Presence of LN metastases did not show any statistically significant impact on the formation of SL. There was a significantly higher incidence of SL in patients who had a higher number of LNs removed $(p<0.001)$. Interestingly, high BMI did not show a significantly higher incidence of SL. Patients affected by SL showed a median of BMI $23.6(22-26) \mathrm{kg} / \mathrm{m}^{2}$. Discussion and Conclusion: The primary purpose of PLND is to obtain improved locoregional staging of prostate cancer to better assess the risk of progression after radical prostatectomy, and to identify patients who may benefit from adjuvant treatment. However, a PLND may be therapeutic rather than simply a staging procedure. Retrospective evidence shows that an extended PLND may give a survival benefit not only for patients affected by prostate cancer with LN-positive disease but also for those with LN-negative disease (1-3). Lymphocele formation remains the most common complication related to lymphadenectomy, occurring as a result of lymph leakage from afferent lymphatic channels transected during resection. Recently, Briganti et al. reported that lymphocele rate was significantly higher after extended PLND compared with limited LND, at $10.3 \%$ vs. $4.6 \%$ (1). The majority of laparoscopic and robotic-assisted approaches are transperitoneal. As peritoneal windows are one form of treatment for symptomatic lymphoceles, one would expect a lower rate of lymphocele in patients in whom this approach is used (3). Indeed, Solberg et al. have reported a lower rate of lymphocele in patients undergoing laparoscopic PLND as compared with open PLND (2). The radiologically detected lymphocele rate ranges from $25 \%$ to $61 \%$, with only $2-9 \%$ becoming clinically symptomatic (1, 3 ). The incidence of SL was $4.9 \%$ in the present study; a lower result compared to most published series. The most common sign/symptom seen was infection, leg edema, deep venous thrombosis and hydrocele. Data analysis of literature suggests three plausible factors that could have an impact on the rate of lymphocele development: the surgeon, transperitoneal $v s$. extraperitoneal approach, and number of LNs removed. In this study we observed an inverse correlation of BMI and the formation of SL. The first report about a BMI-related incidence of SL in patients underwent radical prostatectomy was observed by Mundhenk et al. (3). However, the reason why patients with lower BMI are at an increased risk for SL after RARP remains unclear. In conclusion we can say that PLND during RARP can be performed effectively and safely. An extended template is technically feasible with minimal morbidity. The potential benefits of PLND outweigh its morbidity: a) the overall numbers of nodes removed and percentage of positive LNs are not affected by the robotic assistance, b) rates of $\mathrm{LN}$ metastases are not influenced by the number of LNs removed when a consistent extended template is applied, and c) three parameters remain independent factors influencing the formation of SL (surgeon, BMI and LN count; $p<0.001$ ). Further prospective studies are needed to confirm the long-term benefit of an extended PLND on cancer control. 
1 Briganti A, Chun FK, Salonia A, Suardi N, Gallina A, Da Pozzo LF, Roscigno M, Zanni G, Valiquette L, Rigatti P, Montorsi F and Karakiewicz PI: Complications and other surgical outcomes associated with extended pelvic lymphadenectomy in men with localized prostate cancer. Eur Urol 50: 1006-1013, 2006. PMID: 16959399. DOI: 10.1016/j.eururo.2006.08.015

2 Solberg A, Angelsen A, Bergan U, Haugen OA, Viset T and Klepp O: Frequency of lymphoceles after open and laparoscopic pelvic lymph node dissection in patients with prostate cancer. Scand J Urol Nephrol 37: 218-221, 2003. PMID: 12775280.

3 Mundhenk J, Hennenlotter J, Alloussi S, Selbherr D, Martzog JC, Todenhöfer T, Kruck S, Schwentner C, Stenzl A and Schilling D: Influence of body mass index, surgical approach and lymphadenectomy on the development of symptomatic lymphoceles after radical prostatectomy. Urol Int 90: 270-276, 2013. PMID: 23548783. DOI: 10.1159/ 000347043

\section{2}

\section{AUTOPHAGY SUPPRESSION POTENTIATES THE SUNITINIB-DEPENDENT INHIBITION OF CELL GROWTH AND MIGRATION IN KIDNEY CARCINOMA CELLS}

Gianluca Aguiari $^{1}$, Sonia Guzzo ${ }^{1}$, Simone Patergnani $^{2}$, Ippolto Carmelo ${ }^{3}$, Christian Rocca ${ }^{3}$, Paolo Pinton ${ }^{2}$, Andrea Benedetto Galosi ${ }^{4}$ and Lucio Dell' Atti ${ }^{4}$

${ }^{1}$ Department of Biomedical and Surgical Specialty

Sciences, University of Ferrara, Ferrara, Italy;

${ }^{2}$ Department of Morphology, Surgery and Experimental

Medicine, University of Ferrara, Ferrara, Italy;

${ }^{3}$ Division of Urology, University Hospital

"St. Anna", Ferrara, Italy;

${ }^{4}$ Institute of Urology, University Hospital

"Ospedali Riuniti", Ancona, Italy

Introduction: Renal cell carcinoma (RCC) is a heterogeneous group of cancers arising from renal tubular epithelial cells. The most common subtype of RCC is clear cell RCC (ccRCC) that accounts for $70-80 \%$ of cases. Thirty percent of patients with RCC show metastasis at diagnosis and about $30 \%$ of cases develop disease recurrence after surgical resection. Many RCC patients treated with first-line vascular endothelial growth factor (VEGF) inhibitors such as sunitinib and pazopanib develop disease progression and require second-line therapy (1). The resistance to pharmacological treatment could be due to activation of autophagy. In fact, treatment with chemotherapeutic agents may cause metabolic stress that might induce autophagy, limiting the antitumor efficacy of these drugs (2). Despite the fact that autophagy has been widely implicated as a form of cell death, especially in early stages of tumorigenesis, its role in cancer remains controversial. Cancer cells can use autophagy to survive in hostile metabolic microenvironment conditions, using autophagic substrates to sustain tumor growth and expansion (2). Therefore, the inhibition of autophagy could be used as a tool for the treatment of kidney carcinoma. Moreover, the suppression of this process could also enhance the effectiveness of conventional therapy. Materials and Methods: This study was performed by using kidney carcinoma cells (KJ29 and Caki-2) and SW-40 transformed normal epithelial kidney cells (4/5), used as control (3). The inhibition of autophagy was conducted infecting normal and ccRCC cells with lentiviruses containing the empty pLKO vector, used as control and the 394 and 395 recombinant pLKO vectors expressing specific shRNAs able to silence the autophagic ATG7 gene. The efficiency of ATG7 gene silencing as well as the inhibition of autophagy were evaluated by Western blotting by using specific antibodies for ATG7 and LC3II proteins, respectively. Cell proliferation was investigated by CellTiter cell proliferation assay (3). Briefly, normal (4/5) and ccRCC (KJ29 and Caki-2) cells were infected with either pLKO or 395 lentivirus for $24 \mathrm{~h}$ in DMEM/F12 medium with $1 \%$ FBS. Next, cells were cultured for further $48 \mathrm{~h}$ in the presence of $10 \mu \mathrm{M}$ sunitinib or DMSO (vehicle) and processed following the manufacturer's protocol. Cell migration was evaluated in Caki-2 ccRCC cells, treated as described above. Before the treatment with sunitinib or DMSO, pLKO and 395 transduced cells were grown at confluence in 24-well plates and a groove between the cells was generated using a sterile tip. Cell migration (groove filling) was analyzed after $48 \mathrm{~h}$ of culture with a phase contrast microscope equipped with a CCD camera and processed through the ImageJ software. Apoptosis was analyzed by the Hoechst method (3). Apoptotic nuclei were visualized by a fluorescence microscope after Hoechst staining of Caki- 2 ccRCC cells treated with pLKO and 395 lentiviruses alone or in combination with sunitinib. Images were acquired by a CCD camera and processed using the ImageJ software. Statistical analysis was performed by ANOVA test. Results and Discussion: Because some advanced tumors have elevated levels of autophagy, it has been proposed as a form of tumor maintenance or promotion. Consistently, we have already described that ccRCC cells exhibit higher levels of autophagy than normal kidney cells. Therefore, increased autophagy could correlate with the progression of kidney carcinoma and it could be considered as a new prognostic marker for renal cancer. However, we have observed increased levels of autophagy not only in metastatic ccRCC tissues but also in some non-metastatic kidney tumors (data not shown), therefore autophagy could not be sufficient to promote tumor progression in kidney cancer. To evaluate the involvement of autophagy in the growth and progression of renal tumors, 

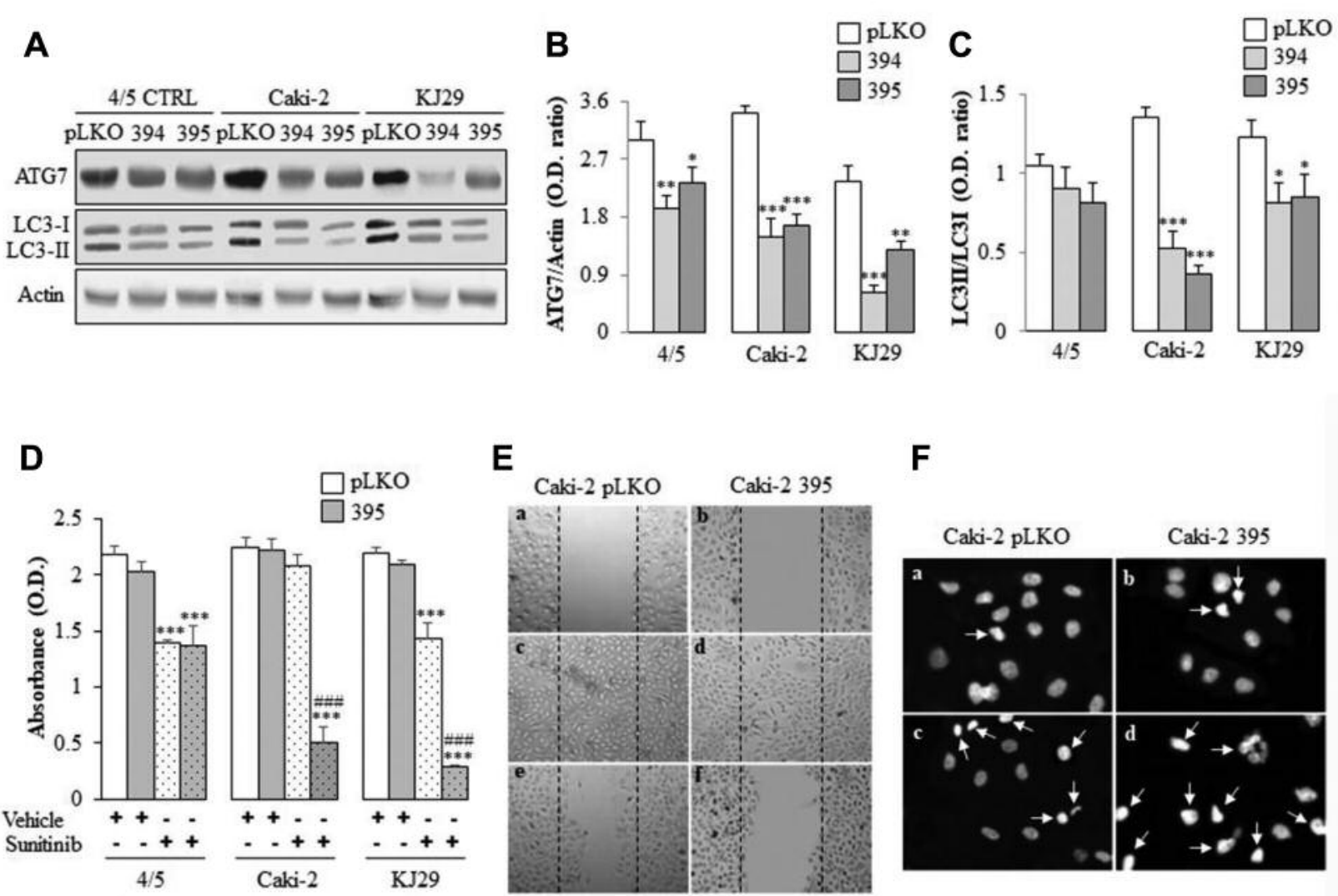

Figure 1. Inhibition of autophagy improves the efficacy of sunitinib causing the reduction of cell proliferation, the suppression of migration and the increase of apoptosis in ccRCC cells. Western blot analysis of normal (4/5) and ccRCC (Caki-2 and KJ29) cells shows the reduction of ATG7 ( $A$ and B) and LC3II ( $A$ and C) proteins in cells transduced with 394 and 395 (ATG7-silencing) lentiviruses compared to same cells transduced with $p L K O$ (control) lentivirus. The statistical significance is $* p<0.05$, $* * p<0.01$ and $* * * p<0.001$. (D) Normal and ccRCC cells were seeded at density of 5000 cells/well in a 96 well plate and infected with pLKO and 395 lentiviruses for 24 h. Next, cells were cultured for further $48 \mathrm{~h}$ in DMEM/F12 1\% FBS in presence of vehicle (DMSO) or sunitinib (10 $\mu M)$ and analyzed by CellTiter method to evaluate cell growth. The treatment with sunitinib significantly reduced cell proliferation in all cell types except for Caki-2 cells $\left({ }^{* *} p<0.001\right)$. The combined treatment with 395 shRNA and sunitinib strongly reduces cell growth in both Caki-2 and KJ29 ccRCC cells

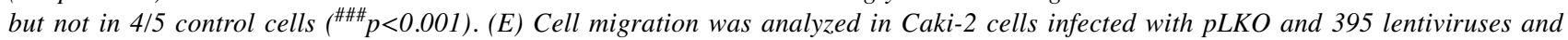
cultured at 100\% confluence in 24 well plates. Before the treatments, Caki-2 pLKO and 395 transduced cells were detached in order to generate a groove (panels $A$ and B, respectively). Then, cells were cultured for further $48 \mathrm{~h}$ in DMEM/F12 $1 \%$ FBS medium in presence of DMSO (panels $C$ and $D$ ) and $10 \mu M$ sunitinib (panels $E$ and $F$ ). Images were acquired at $\times 10$ magnification. $(F)$ Caki-2 cells were treated with pLKO and 395, as described above and cultured on glass coverslips in 6 well plates for 48 h in DMEM/F12 $0.4 \%$ BSA in presence of DMSO (panels $A$ and $B$ ) or with $10 \mu M$ sunitinib (panels $C$ and $D$ ). Images were acquired at $40 x$ magnification. The white arrows indicate the apoptotic nuclei. Data were obtained from at least two independent experiments.

normal (4/5) and ccRCC (KJ29 and Caki-2) cells were infected with recombinant viruses containing specific shRNAs (394 and 395) able to inhibit autophagy by silencing of ATG7gene. As shown in Figure 1A and B, the infection of 4/5 as well as KJ29 and Caki-2 cells with 394 and 395 viruses significantly reduces the levels of ATG7 protein compared with cells transduced with the control virus (pLKO). Consistently, the expression of the autophagic marker LC3II was decreased in 394 and 395 transduced cells, suggesting that the silencing of ATG7 gene causes the inhibition of autophagy in particular in Caki-2 and KJ29 ccRCC cells (Figure 1A and
C). We have observed that the inhibition of autophagy does not significantly change cell growth in normal and ccRCC cells (Figure 1D, gray bars). However, it has been postulated that the cancer cell could use autophagy to trap and degrade molecular drugs by lysosomal vesicles. Thus, the inhibition of autophagy should restore drug response. Consistently, the reduction of autophagy combined with sunitinib treatment, dramatically inhibits cell growth in both Caki-2 and KJ29 ccRCC cells compared with same cells untreated or treated with sunitinib alone (Figure 1D, gray dotted bars). Moreover, the combination between the suppression of autophagy and the 
treatment with sunitinib in Caki-2 cells causes a greater inhibition of cell migration (Figure 1E, box $\mathrm{f}$ ) compared to cells treated with sunitinib alone (Figure 1E, box e). In addition, the reduction of autophagy in Caki-2 cells improves the efficacy of sunitinib increasing the formation of apoptotic nuclei (Figure 1F, box d) compared with cells only treated with sunitinib (Figure 1F, box c). On the contrary, the only suppression of autophagy causes only small effects on cell migration and does not seem to be involved in the activation of apoptosis (Figure 1E box $\mathrm{d}$ and Figure $1 \mathrm{~F}$ box $\mathrm{b}$, respectively). These findings indicate that the inhibition of autophagy does not affect cell growth, migration and apoptosis in kidney cancer cells. However, the reduction of autophagy in combination with sunitinib treatment strongly reduces cell proliferation and migration as well as promotes apoptosis, removing the resistance of kidney cancer cell to the treatment with tyrosine kinase inhibitors. Conclusion: The data reported herein suggest that inhibition of autophagy potentiates the treatment with sunitinib, therefore, this biological process may induce drug resistance in kidney carcinoma cells. Moreover, suppression of autophagy combined with administration of anti-tyrosine kinase drugs could open new roads for the treatment of advanced kidney carcinoma.

1 Yip SM, Wells C, Moreira R, Wong A, Srinivas S, Beuselinck B, Porta C, Sim HW, Ernst DS, Rini BI, Yuasa T, Basappa NS, Kanesvaran R, Wood LA, Canil C, Kapoor A, Fu SYF, Choueiri TK and Heng DYC: Checkpoint inhibitors in patients with metastatic renal cell carcinoma: Results from the international metastatic renal cell carcinoma database consortium. Cancer 124(18): 36773683, 2018. PMID: 30307610. DOI: 10.1002/cncr.31595.

2 Bishop E and Bradshaw TD: Autophagy modulation: a prudent approach in cancer treatment? Cancer Chemother Pharmacol 82(6): 913-922, 2018. PMID: 30182146. DOI: 10.1007/s00280-018-3669-6.

3 Mangolini A, Bonon A, Volinia S, Lanza G, Gambari R, Pinton P, Russo GR, Del Senno L, Dell'Atti L and Aguiari G: Differential expression of microRNA501-5p affects the aggressiveness of clear cell renal carcinoma. FEBS Open Bio 4: 952-965, 2014. PMID: 25426415. DOI: 10.1016/ j.fob.2014.10.016

\section{3}

\section{SBRT FOR LYMPH NODE METASTASES FROM PROSTATE CANCER: A MULTI-INSTITUTIONAL RETROSPECTIVE ANALYSIS}

\author{
Filippo Alongi ${ }^{1}$, Ciro Franzese ${ }^{2}$, Luca Nicosia ${ }^{1}$, \\ Giuseppe D’agostino ${ }^{2}$, Lucia Di Brina ${ }^{2}$, \\ Vanessa Figlia $^{1}$, Rosario Mazzola ${ }^{1}$, Michele Rigo ${ }^{1}$, \\ Stefano Tomatis ${ }^{2}$ and Marta Scorsetti ${ }^{2}$
}

${ }^{1}$ Radiation Oncology, IRCCS Sacro Cuore

Don Calabria Hospital, Negrar-Verona, Italy;

${ }^{2}$ Radiotherapy and Radiosurgery Department, Humanitas

Clinical and Research Hospital, Milan-Rozzano, Italy

Background: Metastases-directed treatment is an emerging strategy for oligometastatic/oligorecurrent/oligoprogressive lymph node metastases from prostate adenocarcinoma. Aim of the present study was to evaluate outcome of patients treated with stereotactic body radiation therapy (SBRT) on lymph node metastases. Materials and Methods: This is a multi-institutional retrospective analysis, including patients affected by lymph node metastases from prostate adenocarcinoma treated with SBRT. Patients with a maximum of 5 lymph node metastases were included. Concomitant treatment with systemic therapy was allowed. End-points of the analysis were local control (LC), out-of-field progressionfree survival (OFPFS), overall progression-free survival (PFS) and overall survival (OS). Results: Eighty patients and 157 lymph node metastases, treated from 2009 to 2018 were evaluated. Median age was 70.2 years and median PSA before SBRT was $1.88 \mathrm{ng} / \mathrm{ml}$. Median diameter of treated lesion was $37 \mathrm{~mm}$ (range 7-40 mm). Dose delivered ranged from 25 to $48 \mathrm{~Gy}$ in 5 to $12 \mathrm{~Gy}$ per fractions (median BED3Gy 116.67, range=66.67-240). Androgen-deprivation therapy was administered concomitantly in 72 lesions. With a median follow-up of 16 months, LC rates at 1 and 3 years were $93 \%$ and $86 \%$. In-field progression of disease was observed in $11(7 \%)$ lesions. One and 3-years OFPFS were $59 \%$ and $29 \%$ while PFS were $49 \%$ and $20 \%$. Median values of OFPFS and PFS were 15 and 11 months, respectively. Rates of OS at 1- and 3-years were $100 \%$ and $95 \%$. Conclusion: SBRT in the management of lymph node metastases from prostate cancer seems to be an effective approach with high rates of in-field control. Prospective trials are necessary to better select patients who can benefit the most from this ablative focal treatment.

\section{4 \\ UPDATED RESULTS OF A PHASE II STUDY ON 5 FRACTIONS FFF SBRT FOR LOW AND INTERMEDIATE PROSTATE CANCER}

Filippo Alongi, Luca Nicosia, Rosario Mazzola, Niccolò Giaj-levra, Francesco Ricchetti, Vanessa Figlia, Michele Rigo, Gianluisa Sicignano, Stefania Naccarato and Ruggero Ruggieri

Radiation Oncology Division, Sacro Cuore Don Calabria Cancer Care Center, Negrar-Verona, Italy

Background/Aim: SBRT has been shown to be a potential treatment option for localized prostate cancer (PC) in a 
selected population. Usually, prostate SBRT has been delivered every other day in order to favor normal tissue recovery, thus minimizing side-effects. Flattening filter free (FFF) delivery is a treatment modality able to reduce treatment beam-on time, decreasing patient positioning uncertainties. We reported feasibility, side effects and biochemical control of FFF SBRT delivered in 5 consecutive days in a cohort of patients affected by localized PC. Patients and Methods: The study, approved by the Ethical Committee, started on January 2014. Inclusion criteria were: age $\leq 80$ years, World Health Organization performance status $\leq 2$, histologically-proven prostate adenocarcinoma, low-to-intermediate risk according to D'Amico criteria, no distant metastases, no previous surgery other than TURP, no other malignant tumor in the previous 5 years, a pre-SBRT International Prostatic Symptoms Score (IPSS) ranged between 0 and 7. The SBRTschedules were: 35 Gy for low risk and 37.5 Gy for intermediate risk PC in 5 fractions, delivered in 5 consecutive days. SBRT was delivered with volumetric modulated radiation therapy (VMAT). Toxicity assessment was performed according to CTCAE v4.0 scale. Neoadjuvant/ concomitant hormonal-therapy was prescribed according to risk classification. Results: Fifty-two patients were enrolled at the time of analysis. Median age was 73 years (55-83), Median follow-up was 33 months (range=6-55 months). Thirty-four (65.3\%) had a low-risk PC and 18 (34.6\%) an intermediate-risk PC. Median initial PSA was $5.9 \mathrm{ng} / \mathrm{ml}$ (range $=1.8-15.7 \mathrm{ng} / \mathrm{ml}$ ). Median Gleason score was 6 (6-7). Median IPSS pre-SBRT was 4.5 (range=0-7). All patients completed the treatment as planned. Acute G1-2 toxicity occurred in $18(34.6 \%)$ patients and was distributed as follows: $8(15.3 \%)$ cases of G1 gastrointestinal toxicity, 1 (1.9\%) patients had G2 gastrointestinal toxicity, 5 (9.6\%) patients reported G2 genitourinary toxicity and $11(21.1 \%)$ G1 genitourinary toxicity. Patients may have experienced more than one toxicity. Late G1 gastrointestinal toxicity occurred in $5(9.6 \%)$ patients. No G3 toxicities occurred. At the last follow-up median IPSS was 3 (1-19) and median PSA was $0.315 \mathrm{ng} / \mathrm{ml}$ (range=0.04-7.965 ng/ml). Biochemical control was $98 \%$. Conclusion: The results of our study showed that FFF SBRT in 5 fractions for low-to-intermediate PC is feasible and well tolerated. Longer follow-up is necessary to assess late toxicity and long-term effectiveness.

\author{
15 \\ POST-OPERATIVE COMPLICATIONS IN RADICAL \\ CYSTECTOMY: CAN THE KIND OF \\ URINARY DIVERSION, THE PERFOMANCE \\ STATUS, PRESENCE OF PREOPERATIVE \\ HYDRONEPHROSIS OR TUMOR EXTENSION \\ PREDICT THE LIKELIHOOD OF POST- \\ OPERATIVE COMPLICATIONS?
}

$\underline{\text { Nicolò Fiorello }}^{1}$, Hussein Jallous ${ }^{1}$, Carlo Marchetti ${ }^{1}$, Giovanni Luca Giliberto ${ }^{1}$, Andrea Ringressi ${ }^{1}$, Daniele Porru ${ }^{1}$, Tiziano Cebrelli ${ }^{1}$ and Claudio Simeone ${ }^{2}$

${ }^{1}$ Division of Urology, IRCCS Policlinic

Hospital S. Matteo Pavia, Pavia, Italy;

${ }^{2}$ Division of Urology, University of Brescia, Hospital Spedali Civili, Brescia, Italy

Introduction: Radical cystectomy ( $\mathrm{RC}$ ) is recommended for T2-4 N0 M0 bladder cancer and high-risk non-muscle invasive bladder cancer. Despite improvements in the surgical technique, the procedure remains associated with significant postoperative morbidity with high rates of complications. Materials and Methods: In this study we have enrolled 213 patients, 171 men and 42 women, and we have evaluated the anatomical features, the comorbidities, clinical extension of tumor to plane the surgery. The average age is 74.52 years. For every patient regional or extended lymphadenectomy was performed. The kinds of urinary diversion are been ureterocutaneostomy (UCS), ureteroileocutaneostomy (UICS, with Wallace I/II or Bricker technique), or neobladder. Performance status has been evaluated with the Charlson score. Seventy-five patients had preoperative hydronephrosis. In most cases it was due to the extension of the tumor at bladder trigone. Results: We looked for any relations with the performance status of patients, classified according to the Charlson score, but the rate of complications was not directly related to the comorbidity of patients. Despite that neobladder is related to a high rate of complications, in this study the surgical complexity was not directly related to the rate of compliance. Preoperative hydronephrosis was related in most cases to local extension of tumor. In 7 patients hydronephosis was provoked by previous TURB of tumors that involved the ureteral ostium. We have not found relations between preoperative hydronephrosis and post-operative complications. Local extension of tumor certainly makes the intervention more complex, but we have not found a direct relation between local extension and post-operative complications. Conclusion: The radical cystectomy is an invasive treatment reserved to muscleinvasive tumors of the bladder and for patients who do not have excessive comorbidity. A further selection of patients is necessary to choose the kind of urinary diversion and requires great experience of surgeon. Despite the improvement of surgical skills of urologists, the radical cystectomy remains an invasive treatment with a high rate of complications, which must be managed in the most appropriate way. In our study, according to literature, it was not possible found a way to predict the rate of postoperative complications. 
16

\section{ADJUVANT AND SALVAGE RADIOTHERAPY FOR PROSTATE CANCER IN EVERYDAY CLINICAL PRACTICE: DIFFERENCES BETWEEN A TERTIARY AND A PRIVATE HIGH-VOLUME CENTER}

Davide Baiamonte $^{1}$, Vincenzo Serretta ${ }^{1}$, Billeci Sandro ${ }^{1}$, Scalici Gesolfo Cristina' ${ }^{2}$, Ivan $\mathrm{Fazio}^{3}$, Manuela Federico ${ }^{3}$, Mazzonello Antonella ${ }^{3}$, Gianluca Mortellaro ${ }^{4}$,

Giuseppe Ferrera ${ }^{4}$, Francesco Cuccia ${ }^{5}$,

Chiara Sanfilippo $^{6}$ and Members

of the GSTU Foundation ${ }^{7}$

${ }^{1}$ Section of Urology, Department of Surgical, Oncological and Oral Sciences, University of Palermo, Palermo, Italy; ${ }^{2}$ Department of Urology, Paolo Borsellino

Hospital, Marsala, Italy;

${ }^{3}$ Radiotherapy Unit, Macchiarella Clinic, Palermo, Italy;

${ }^{4}$ Radiation Oncology Unit, ARNAS

Civico Hospital, Palermo, Italy;

${ }^{5}$ Radiation Oncology School, University

of Palermo, Palermo, Italy;

${ }^{6}$ Statistics, GSTU Foundation, Palermo, Italy;

${ }^{7}$ Other Members, GSTU Foundation, Palermo, Italy

Introduction: Radiotherapy plays a primary role as adjuvant radiotherapy (AR) after radical prostatectomy in high-risk prostate cancer and as salvage radiotherapy (SR) at local/biochemical recurrence. EAU/ESTRO international guidelines recommend patient's selection criteria and PSA levels for both treatments. Nevertheless, the clinical scenarios in everyday clinical practice remain unknown. The differences emerging in everyday clinical practice between a tertiary and a private high-volume radiotherapy center of the same city and the divergences from the European guidelines were retrospectively investigated. Materials and Methods: Unselected, consecutive patients treated between 2015 and 2017 with AR or SR after radical prostatectomy were enrolled in this study. A database was created including patients' characteristics, PSA levels before radiotherapy, Gleason Score, pTNM, surgical margins' status, concomitant hormonotherapy, treatment's dose, technique and schedule. PSA and adverse genitourinary (GU) and gastrointestinal (GI) events were recorded up to 3 months after radiotherapy (CTCAE scale v4.03) to evaluate toxicity and efficacy of the therapies. Inclusion criteria for adjuvant and salvage radiotherapy were compared between the two centers and with EAU-ESTRO guidelines. Statistical analysis was performed by R v3.4.2 software. Results: A total of 96 patients were enrolled, $58(60.4 \%)$ treated with adjuvant radiotherapy and $38(39.6 \%)$ with salvage radiotherapy in two radiotherapy centers (Center A and Center B) of the same city. There were no statistically significant differences
Table I. Patient $p N$ and surgical $R$ margins.

\begin{tabular}{|c|c|c|c|c|}
\hline & \multicolumn{2}{|c|}{ Adjuvant RT } & \multicolumn{2}{|c|}{ Savage RT } \\
\hline & Center A & Center B & Center A & Center B \\
\hline N. patients & 27 & 31 & 23 & 15 \\
\hline \multicolumn{5}{|l|}{$\mathrm{pN}(\%)$} \\
\hline No & $17(63.0 \%)$ & $16(51.6 \%)$ & $13(56.5 \%)$ & $11(73.4 \%)$ \\
\hline $\mathrm{N} 1$ & $7(25.9 \%)$ & $11(35.5 \%)$ & $2(8.7 \%)$ & $2(13.3 \%)$ \\
\hline $\mathrm{Nx}$ & $3(11.1 \%)$ & $4(12.9 \%)$ & $8(34.8 \%)$ & $2(13.3 \%)$ \\
\hline \multicolumn{5}{|l|}{$\begin{array}{l}\text { R Margins } \\
(\%)\end{array}$} \\
\hline R0 & $10(37.0 \%)$ & $12(38.7 \%)$ & $15(65.2 \%)$ & $8(53.3 \%)$ \\
\hline \multirow[t]{2}{*}{$\mathrm{R} 1$} & $17(63.0 \%)$ & $19(61.3 \%)$ & $8(34.8 \%)$ & $7(46.7 \%)$ \\
\hline & \multicolumn{2}{|c|}{$\begin{array}{c}p \text {-value } \mathrm{pN}=1 \\
p \text {-value } \mathrm{pN}=0.69\end{array}$} & \multicolumn{2}{|c|}{$\begin{array}{l}p \text {-value } \mathrm{R}=0.67 \\
p \text {-value } \mathrm{R}=0.34\end{array}$} \\
\hline
\end{tabular}

Table II. Patient treatment characteristics.

\begin{tabular}{|c|c|c|c|c|}
\hline & \multicolumn{2}{|c|}{ Adjuvant RT } & \multicolumn{2}{|c|}{ Savage RT } \\
\hline & Center A & Center B & Center A & Center B \\
\hline N. patients & 27 & 31 & 23 & 15 \\
\hline \multicolumn{5}{|l|}{ Dose (Gy) } \\
\hline Mean & 63.7 & 72.5 & 64.11 & 71.49 \\
\hline Median & 63.8 & 72.0 & 63.8 & 72.0 \\
\hline Range & $60.9-64.4$ & $66.0-78.4$ & $63.8-66$ & $66-76$ \\
\hline \multicolumn{5}{|c|}{$\begin{array}{l}\text { N. sessions } \\
(\mathrm{dd})\end{array}$} \\
\hline Mean & 29 & 36 & 29 & 37 \\
\hline Median & 29 & 37 & 29 & 37 \\
\hline Range & $28-30$ & $28-42$ & $28-30$ & $33-39$ \\
\hline \multicolumn{5}{|l|}{$\begin{array}{l}\text { Duration } \\
\text { (dd) }\end{array}$} \\
\hline Mean & 44 & 53 & 42 & 54 \\
\hline Median & 43 & 54 & 43 & 52 \\
\hline Range & $38-51$ & $37-64$ & $32-48$ & $47-61$ \\
\hline \multicolumn{5}{|c|}{$\begin{array}{l}\text { Hormono- } \\
\text { therapy }(\%)\end{array}$} \\
\hline Yes & $12(44.5 \%)$ & $17(54.8 \%)$ & $11(47.8 \%)$ & $10(66.7 \%)$ \\
\hline No & $15(55.5 \%)$ & $14(45.2 \%)$ & $12(52.2 \%)$ & $5(33.3 \%)$ \\
\hline
\end{tabular}

between the two centers regarding in incidence of positive surgical margins R1 (AR $p=1$; SR $p=0.69$ ) and in lymph nodes involvement $\mathrm{pN}$ (AR $p=0.67$; SR $p=0.34$ ) (Table I). Hormonotherapy was administered in $29(50 \%)$ patients treated with AR and in $21(55.2 \%)$ patients treated with SR. There was a significative difference in pre-adjuvant radiotherapy PSA levels, with higher values in Center B (median PSA $0.03 \mathrm{ng} / \mathrm{ml} v s .0 .2 \mathrm{ng} / \mathrm{ml}, p=0.012$ ) (Table II) 

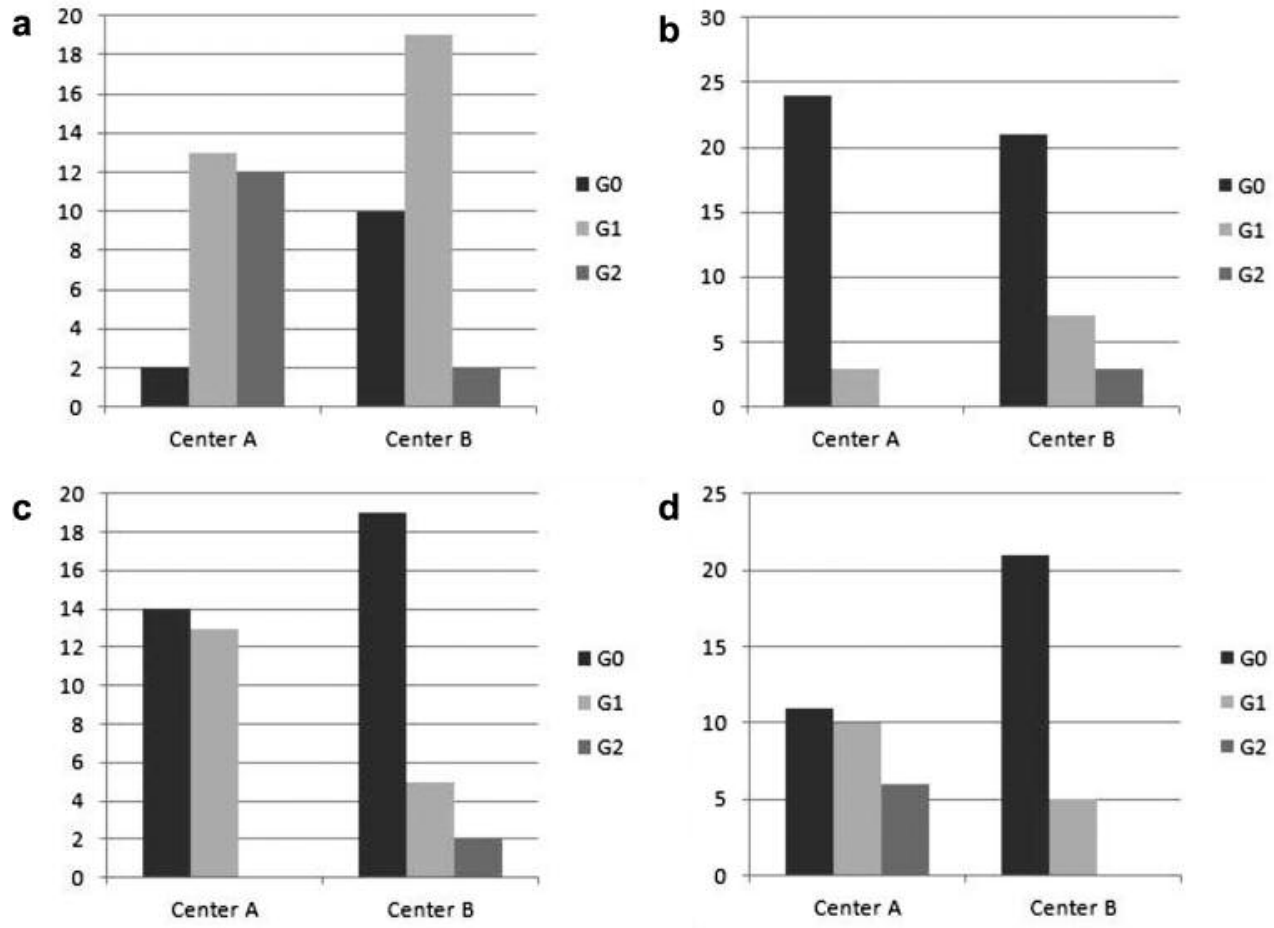

Figure 1. GU and GI side-effects in adjuvant groups.

a

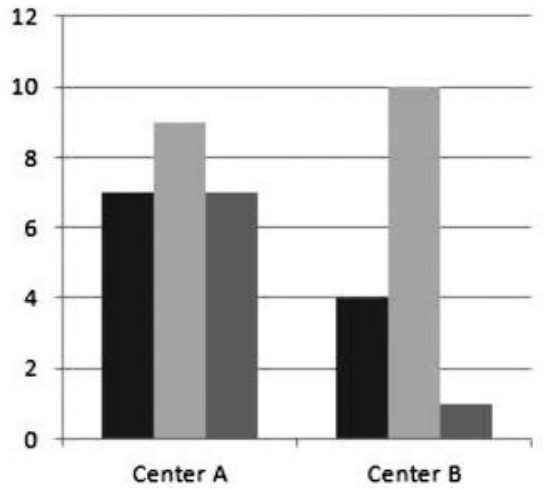

C

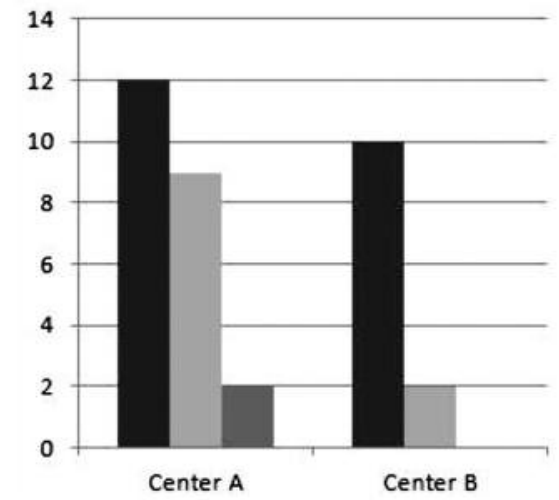

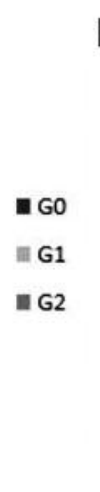
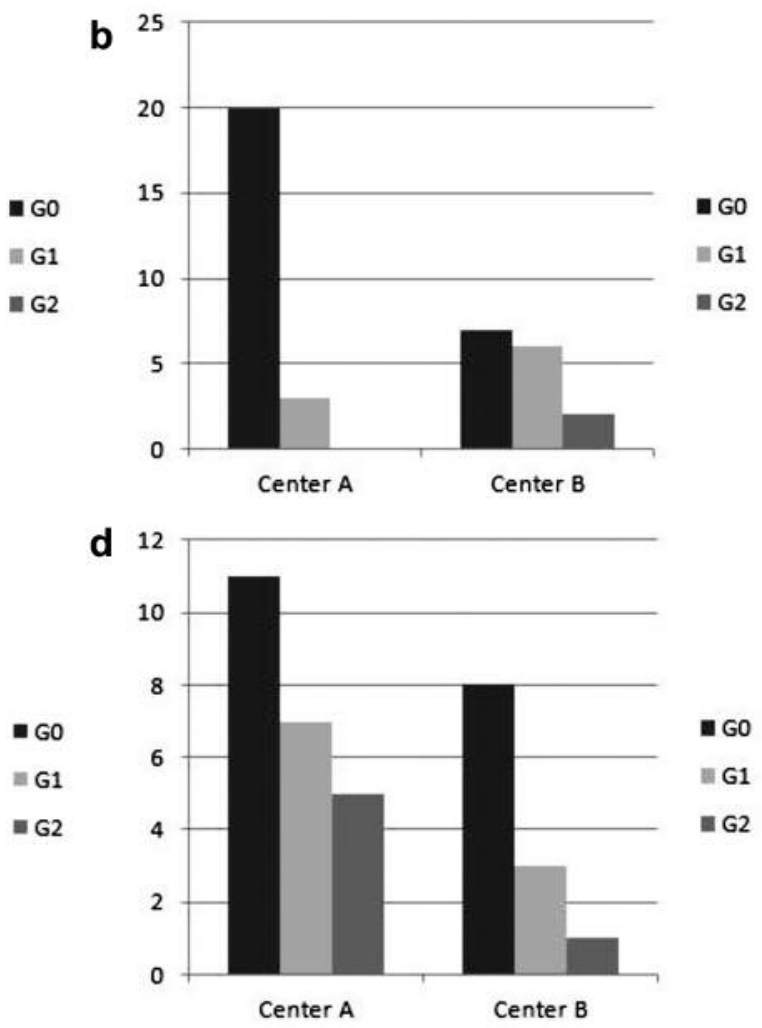

Figure 2. GU and GI side-effects in salvage groups. 
while PSA levels in pre-savage groups were similar, with a trend of higher values in Center B (median PSA $0.71 \mathrm{ng} / \mathrm{ml}$ vs. $2.2 \mathrm{ng} / \mathrm{ml}, p=0.094)$. Center A delivered lower doses (median dose $63.8 \mathrm{~Gy}$ vs. $72 \mathrm{~Gy}$ ) with higher fractionation (2.2 Gy vs. $2.0 \mathrm{~Gy}$ ), with a reduced length of treatment (43 vs. 52 days). Median PSA values at 3 months were less than $0,1 \mathrm{ng} / \mathrm{ml}$ in both centers both for $\mathrm{AR}$ and SR, resulting in comparable and optimal efficacies. Lower GU toxicity was detected in Center B for patients treated with AR (GU $p=0.02$; GI $p=0.45$ ) while the difference in SR group was not statistically significant (GU $p=0.16$; GI $p=0.53$ ) (Figures 1 and 2). No Grade 3 or 4 adverse effects were recorded. Comparing the inclusion criteria, several differences emerged between the two centers and with the EAU-ESTRO guidelines. According to EAU/ESTRO criteria, out of the 58 treatments classified in "real-life" practice as adjuvant treatment, $29(50 \%)$ would be categorized as savage radiotherapy. Discussion and Conclusion: Marked differences in terms of criteria, PSA levels at treatment start, technical equipment, timing, duration and dose emerged between two centers of the same city. Moreover, both centers strikingly diverged from guidelines. Despite the abovementioned variances, no clinically relevant differences emerged in efficacy in terms of PSA at 3 months both in adjuvant and salvage radiotherapy. Slight differences emerged in short-term genitourinary Grade 1-2 toxicity in adjuvant treatments, while no difference was found comparing savage groups.

Thanks to the GSTU Foundation for the editing and statistical and support.

17

\section{MULTIPARAMETRIC MAGNETIC RESONANCE IMAGING PRIOR TO SALVAGE RADIOTHERAPY FOR BIOCHEMICAL RECURRENCE AFTER RADICAL PROSTATECTOMY}

Suela Vukcaj ${ }^{1}$, Giovanna Perugini ${ }^{2}$, Roberta Muni ${ }^{1}$, Lucia Feltre $^{1}$, Francesco Filippone ${ }^{1}$, Eva Iannacone ${ }^{1}$, Maurizio Kalli ${ }^{1}$, Laura Maffioletti ${ }^{1}$, Fabio Piccoli ${ }^{1}$, Giulia Querques ${ }^{2}$, Marco Roscigno ${ }^{3}$, Luigi Da Pozzo ${ }^{3}$, Sandro Sironi ${ }^{2}$ and Luigi Franco Cazzaniga ${ }^{1}$

${ }^{1}$ Radiotherapy Unit, Papa Giovanni

XXIII Hospital, Bergamo, Italy;

${ }^{2}$ Radiology, Papa Giovanni XXIII Hospital, Bergamo, Italy; ${ }^{3}$ Urology, Papa Giovanni XXIII Hospital, Bergamo, Italy

Introduction: Radical prostatectomy in patients with clinically localized prostate cancer is an effective treatment, but may fail in up to $20-40 \%$ of cases. The possible patterns of postprostatectomy relapse can be local recurrence in the prostatic bed, regional recurrence in the lymph nodes, metastatic disease, or a combination of these. For salvage radiotherapy to be most effective, treatment should be considered before the PSA level is allowed to rise too high, when disease is more likely to be confined to the prostate bed. At low PSA levels, current imaging techniques are poor at detecting disease, making it difficult to differentiate local and distant recurrences and to target the radiotherapy appropriately. Usually, the site of recurrence is unknown and salvage radiotherapy target volumes of the prostatic bed are based on empirical data and differ between different guidelines. In recent years, multiparametric magnetic resonance imaging (MPMRI) has become more widely used in these settings of patients and it is the only imaging technique recommended by the European Society of Urogenital Radiology to evaluate pelvic recurrence in patients with low PSA levels. Materials and Methods: We reviewed data on 30 patients with biochemical relapse after radical prostatectomy who underwent to 3 Tesla MPMRI with T2-weighted imaging, diffusion weighted imaging (DWI) and dynamic contrast-enhanced imaging (DCE), from April 2016 to October 2018 at the Papa Giovanni XXIII Hospital of Bergamo. Target volume dose was $70 \mathrm{~Gy} / 35$ fractions, homogenously, on prostatic bed in patients without macroscopic lesions on MPMRI while, patients with macroscopic recurrence detected by MPMRI received an integrated boost up to $72 \mathrm{~Gy} / 32$ fractions at the recurrent tumor volume and $64 \mathrm{~Gy} / 32$ fractions in the remaining prostate bed. We used a Chi-Square test to identify any possible relationship between clinical, pathological and MPMRI results. Results: Median value of PSA at the time of biochemical relapse was $0.25 \mathrm{ng} / \mathrm{ml}$ (range $=0.10-0.67 \mathrm{ng} / \mathrm{ml}$ ). MPMRI was positive in $33.3 \%$ of patients. In 1 patient MPMRI showed, at the level of bladder-ureteral anastomosis, a vegetative tissue thickening in the bladder lumen which was histologically confirmed to be normal prostatic tissue, so no salvage radiotherapy was performed. In another patient, a dubious macroscopic recurrence in the prostate bed and a bone metastasis was detected, subsequently not confirmed by PET choline, so salvage radiotherapy was performed as standard. Median PSA value in positive MPMRI was $0.24 \mathrm{ng} / \mathrm{ml}$ (range $=0.15-0.67 \mathrm{ng} / \mathrm{ml}$ ) and median PSA doubling time was 7.84 months (range=1.77-20.76 months) while, in patients without macroscopic recurrence at MPMRI median PSA values was $0.26 \mathrm{ng} / \mathrm{ml}$ (range $=0.10-0.54 \mathrm{ng} / \mathrm{ml}$ ) and median PSA doubling time was 7.54 months (range=1.55-22.32 months). Median PSA velocity was $0.02 \mathrm{ng} / \mathrm{ml} / \mathrm{y}$ for both positive and negative MPMRI patients. The perianastomotic site was the most common location of local recurrence documented by MPMRI. Most of patients had pT2c or pT3, pN0 tumors and $70 \%$ of patients had Gleason score $7.60 \%$ of patients had positive surgical margins. We did not find statistically significant differences in clinical and pathological variables between patients with positive and negative MPMRI. Conclusion: MPMRI could detect the macroscopic site of 
recurrence nearly in one third of cases (despite low PSA levels) and could be used to counteract target omissions and improve the accuracy and precision of dose delivery in salvage radiotherapy after radical prostatectomy. Our results suggest that the information obtained by MPMRI could influence diagnostic and therapeutic choice and could be integrated into the decision-making process for salvage radiotherapy planning.

Further prospective trials are necessary to investigate the utility of MPMRI in salvage radiotherapy as well as the role of dose escalation-de-escalation protocols.

\section{8 \\ ACTIVE SURVEILLANCE IN NON-MUSCLE INVASIVE BLADDER CANCER: A SINGLE CENTER RETROSPECTIVE EXPERIENCE}

Marco Ennas, Antonia di Domenico,

Stefania Tamagno, Fabio Bonini, Fabio Campodonico,

Giorgio Canepa and Carlo Introini

Department of Urology, Ente Ospedaliero Ospedali

Galliera, Genoa, Italy

Introduction: The standard treatment for non-muscle invasive bladder cancer (NMIBC) is the transurethral resection of bladder tumor (TURB-T). Because of the high recurrence rate of disease, many patients undergo multiple surgery. Nevertheless TURB-T may bear the risk of complications such as bleeding, urinary tract infections, bladder perforations or urethral stricture; moreover, it poses emotional and economical burden and induces additive damage to the bladder (1). Further a high number of patients are old, with several co-morbidities, giving a high risk of anesthesia complications. Finally, low risk bladder cancers have a well-established low risk of progression, repeated TURB-T do not reduce the risk for further tumor recurrence (2). The Aim of Active Surveillance (AS) is to reduce the number of TURB-T throughout patients' lifetime, reducing the burden of disease related to surgery, anesthesia and hospital stay (2). To date only few studies, report outcomes on oncological safety of AS for NMIBC, we report our single center experience (3). Materials and Methods: From September 2006 to July 201894 AS periods were documented in 94 patients (mean age $=69.4$ years; range $=42-87$ years) at a second event of NMIBC. Previous pTa stage were reported in 82 patients $(87 \%)$, pT1 was reported in 12 patients (13\%). A total of 23 patients had a previous low-grade disease, whilst 71 presented high-grade tumors. The presence of carcinoma in situ was an exclusion criterion. AS was offered to patients with a number of lesions ranging from 1 to 6 (mean=1.7). According to the EORTC Tables, the mean risk of recurrence at 5 years was $54.5 \%$ (range $=31-62 \%)$, whereas the mean risk of progression at 5 years was $8 \%$ (range $=6-17 \%$ ).
The mean Charlson morbidity index of patients included was 3 (range=0-6). The AS was started taking into account previous pathological features, the macroscopic characteristics of lesions, patients' co-morbidites and preference in treatment. Informed consent was provided to all patients. The follow-up consisted of cytology and an in-office flexible cystoscopy for male patients and rigid endoscopy for female patients, every three months for the first year and then every six months. The criteria for termination of AS were excessive tumor growth, appearance of additional tumor, changing of macroscopic features and hematuria. Results: One patient was lost at followup, another one underwent in office coagulation and a third one is waiting for the repeated TURB-T, so that we report the results concerning 91 patients. Mean period length was 18.4 months (range=3-64 months). The mean follow-up was 40.7 months. The AS terminated for 78 patients $(85.7 \%), 13$ periods $(14.3 \%)$ are still ongoing. The main reasons for termination were the appearance of additional tumors (39 patients), bleeding (18 patients), and the excessive tumor growth (17 patients), followed by the changing of macroscopic features (4 patients). Among patients that underwent a repeated TURB-T, there was disease progression in 16 patients (17.6\%). No patients progressed to muscle-invasive patients nor presented carcinoma in situ and all patients included in the AS are Radical Cystectomy-free. Discussion and Conclusion: Disclaiming that the main limitation of this study is its retrospective design and the lack of strict inclusion criteria, it seems that AS for NMIBC could be reasonable and safe in patients with low-risk tumors due to the relative low risk of progression and the absence of development of muscle invasive bladder cancer; it allows to reduce the number of hospitalization for TURB-T, delay the discomfort and morbidity and spare bladder volume loss related to repeated surgery.

1 Gofrit ON, Pode D, Lazar A, Katz R and Shapiro A: Watchful waiting policy in recurrent Ta G1 bladder tumors. Eur Urol 49: 306-307, 2006. PMID: 16413659. DOI: 10.1016/j.eururo.2005.12.029

2 Hurle R, Pasini L, Lazzeri M, Colombo P, Buffi N, Lughezzani G, Casale P, Morenghi E, Peschechera R, Zandegiacomo S, Benetti A, Saita A, Cardone P and Guazzoni G: Active surveillance for low risk non-muscle invasive bladder cancer: mid-term results from the Bladder cancer Italian Active Surveillance (BIAS) project. BJU Int 118: 935-939, 2016. PMID: 27207387. DOI: 10.1111/ bju. 13536

3 Oosterlinck W and Decaestecker K: Re: Active surveillance for low risk Non-Muscle Invasive Bladder Cancer (NMIBC): a confirmatory and resource consumption study from Bladder cancer Italian Active Surveillance (BIAS) project. Eur Urol 73: 476-481, 2017. PMID: 29224915. DOI: $10.1016 /$ j.eururo.2017.11.026 
19

\section{ELECTIVE PELVIC NODAL IRRADIATION COMBINED WITH A SINGLE-SHOT BOOST FOR THE FOCAL NODAL IN THE IMAGE-GUIDED VMAT OF OLIGORECURRENT NODAL FAILURES FROM PROSTATE CANCER: PRELIMINARY EXPERIENCE}

\author{
Alessandro Magli ${ }^{1}$, Eugenia Moretti ${ }^{2}$, \\ Fabrizio Tonetto $^{3}$, Paolo Scalchi ${ }^{2}$ and Marco Trovò ${ }^{3}$ \\ ${ }^{1}$ Department of Radiation Oncology, Azienda Sanitaria \\ Universitaria Integrata di Udine (ASUIUD), Udine, Italy; \\ ${ }^{2}$ Department of Medial Physics, Udine, Italy; \\ ${ }^{3}$ Department ofRadiation Oncology, Udine, Italy
}

Aim: Radiation therapy (RT) as salvage treatment for oligometastatic ( $\leq 5$ metastases) pelvic nodal relapses of prostate cancer $(\mathrm{PCa})$ is promising in terms of local control with low treatment-related toxicities. Both stereotactic body RT (SBRT) and elective pelvic nodal RT (ENRT) have been used in this setting and are considered valid, even if still investigational treatment options. However, the best way to irradiate patients with an exclusive oligometastatic nodal disease remains debated. Most subsequent relapses after only SBRT for nodal recurrences are nodal and oligometastatic. The concept of spatial cooperation between SBRT and ENRT is also attractive in this kind of clinical scenario. Hence, in this area we present our preliminary results in terms of early outcomes and toxicity data combining ENRT and SBRT on the pelvic nodal relapses after primary treatment for PCa. Materials and Methods: From April 2017 to March 2018, 12 patients with 15 isolated lymph nodes of recurrent prostate cancer were treated combining ENRT with SBRT on pelvic nodal relapses in single-institution retrospective study of the University Hospital of Udine (ASUIUD). Patient features are summarized in Table I. All patients underwent Choline-PET or PSMA PET/ CT to assess local failure. The prophylactic lymph nodal delineations followed the Radiation Therapy Oncology Group (RTOG) guidelines. A three-dimensional volumetric margin of $5 \mathrm{~mm}$ was grown all around the prophylactic and gross nodal clinical target volume to generate the respective nodal PTV. A SIB schema was designed to deliver $54 \mathrm{~Gy}(1.8 \mathrm{~Gy} /$ fraction) to the pelvic lymph nodals, and $60 \mathrm{~Gy}(2 \mathrm{~Gy} /$ fraction) to the positive nodals. The SBRT treatment was designed to deliver in one session $10 \mathrm{~Gy}$ to the positive nodes. The delivery techniques employed were IMRT and VMAT. The main focus planning was achieving good target coverage (V95 $\geq 95)$ with optimal organ sparing. For organs at risk, the dose constraints used are derived from QUANTEC Neoadjuvant ADT was administered for to all patients for a median time of 3 months. Routine institutional image-based patient position verification protocols foresaw daily on-line matching by
Table I. Patient characteristics ( $N=12 \mathrm{pts})$.

\begin{tabular}{lc}
\hline Characteristic & Value \\
\hline Age, yr & \\
Mean \pm DS & $71 \pm 6$ \\
Median (range) & $72(67-80)$ \\
Primary treatment & \\
EBRT & 1 \\
Surgery & 6 \\
Surgery + EBRT & 5 \\
Stage - N & \\
T2b-T2c & 7 \\
T3a-T3b & 5 \\
Gleason score & \\
$6-\mathrm{N}$ & 2 \\
$7-\mathrm{N}$ & 6 \\
$\geq 8-\mathrm{N}$ & 4 \\
Progression-free survival mo & \\
$<12$ & 0 \\
$>12$ & 12 \\
PET imaging & \\
PSMA-N & 3 \\
Colina-N & 9 \\
Baseline PSA, ng/ml & \\
Median & \\
$\geq 0,5-1-\mathrm{N}$ & 0.9 \\
$\geq 1-\mathrm{N}$ & 6 \\
Number of treated nodes & 6 \\
1 & \\
2 & \\
$\geq 3$ & \\
\hline & \\
\hline & \\
&
\end{tabular}

$\mathrm{SD}=$ standard deviation; $\mathrm{PSA}=$ prostate-specific antigen, $\mathrm{Yr}=\mathrm{years}$; mo=months; $\mathrm{N}=$ number of patients, $\mathrm{PSMA}=$ prostate-specific membrane antigen.

CBCT. The acute and late toxicities were recorded using the RTOG/EORTC scale. Restaging with TC/PET was performed 3 months after the end of treatment. Results: The median follow-up duration was 6 months (range $=3-12$ months). The median age was 70 years (range $=61-78$ years). The median time from primary cancer treatment to ENRT combined with SBRT was 3.4 years (range $=2.4-7.9$ years) with a primary Gleason score of $\geq 8$ in 4 patients. All patients were treated to pelvic nodal site. One and two oligometastatic lesions were treated in $75 \%$ and $25 \%$ of patients, respectively. Median PSA values at the time of the Choline-PET and PSMA PET/ CT were $1.16 \mathrm{ng} / \mathrm{ml}$ and 0.59 $\mathrm{ng} / \mathrm{ml}$, respectively. All patients completed the prescribed radiation treatment, with no interruption. Acute GU toxicity of grade 1 (increase in urinary frequency) was observed in one patient. The incidence of acute GI and GU toxicity of any grade were $14.2 \%$ and $35.7 \%$, respectively. No acute and late toxicity $\geq 2$ was noted. Biochemical response seems to be complete at 3 months and 10 patients underwent a 
restaging with TC/PET 3-6 months after the end of treatment with complete metabolic response. Conclusion: Combined treatment modalities are safe in the treatment of nodal metacrochronous oligometastatic castration-naive prostate cancer after primary treatment of PCa. These results may provide a basis for a larger phase II study to examine the role of the elective pelvic nodal irradiation combined with focal nodal SBRT in this population currently treated only with focal nodal SBRT.

\section{0}

\section{LEARNING CURVE OF URETERO-} NEOCYSTOSTOMY: IN ROBOTIC SURGERY THERE IS A NEW PARAMETER TO EVALUATE

Giuseppe Maiolino ${ }^{1}$, Federico Lanzi ${ }^{1}$, Filippo Gentile ${ }^{1}$, Gerardo Pizzirusso ${ }^{1}$, Tommaso Chini ${ }^{1}$, Gabriele Gaziev ${ }^{1}$, Filippo Cecconi ${ }^{1}$, Gianni Vittori ${ }^{2}$ and Gabriele Barbanti ${ }^{1}$

${ }^{1}$ Department of Urology, Santa Maria

Le Scotte Hospital, Siena, Italy;

${ }^{2}$ Department of Urology, Careggi Hospital, Florence, Italy

Background: Learning curve (LC) is defined as the period for a surgeon to achieve a complete competence about a new specific surgical procedure. A LC is designed by analysis in the time of a one or more several outcomes: many authors chose operating time (OT), estimated blood loss (EBL), rate of complications, biochemical recurrence, etc. Currently, there is not a definition or a classification of LC since many factors can influence this period (surgeon's attitude, intensity of the performed procedures, confidence with a similar procedure with open technique, previous simulations with virtual reality, etc.), and numerous learning curve can be possibly designed on different peri- or post-operative outcomes. Furthermore, a specific procedure has a specific outcome: for example, for radical prostatectomy, the rate of free margins, the continence scores, and the rate of biochemical recurrence can be useful as outcomes, but they are unusable for urethral stenosis. There are no studies in the literature that analyze the learning curve about ureteral surgery, particularly on direct uretero-neocystostomy (1). Patients and Methods: We retrospectively collected the operating time and estimated blood loss of 26 adult patients who underwent ureteroneocystostomy between 2014 and 2018 in the Department of Urology, Santa Maria alle Scotte Hospital, Siena. Thirteen patients received RAUR (robotassisted ureteral reimplantation), while others received OUR (open ureteral reimplantation). Robotic procedures were carried out by a urologist in training regarding the robotic approach to the pathologies of the ureter, while the open procedures were conducted by a urologist with proven experience on ureteral pathologies with an open approach.
Table I. Data for robotic-assisted ureteral reimplantation (RAUR; $n=13)$ and open ureteral reimplantation (OUR; $n=13)$ surgical procedures.

\begin{tabular}{|c|c|c|c|}
\hline Procedure & $\begin{array}{l}\text { Date of } \\
\text { surgery }\end{array}$ & $\begin{array}{l}\text { Operating } \\
\text { time (min) }\end{array}$ & $\begin{array}{c}\text { Estimated blood } \\
\text { loss (ml) }\end{array}$ \\
\hline \multirow[t]{13}{*}{ RAUR } & $02 / 2016$ & 120 & 80 \\
\hline & $03 / 2016$ & 130 & 75 \\
\hline & $04 / 2016$ & 130 & 65 \\
\hline & $06 / 2016$ & 120 & 70 \\
\hline & $10 / 2016$ & 115 & 50 \\
\hline & $08 / 2017$ & 110 & 50 \\
\hline & $08 / 2017$ & 90 & 40 \\
\hline & $12 / 2017$ & 90 & 55 \\
\hline & $12 / 2017$ & 82 & 50 \\
\hline & $02 / 2018$ & 70 & 45 \\
\hline & $05 / 2018$ & 65 & 50 \\
\hline & $05 / 2018$ & 65 & 50 \\
\hline & $05 / 2018$ & 70 & 45 \\
\hline \multirow[t]{13}{*}{ OUR } & $01 / 2014$ & 155 & 200 \\
\hline & $08 / 2014$ & 140 & 250 \\
\hline & $10 / 2014$ & 140 & 320 \\
\hline & $01 / 2016$ & 120 & 235 \\
\hline & $03 / 2015$ & 130 & 250 \\
\hline & $05 / 2015$ & 110 & 240 \\
\hline & $06 / 2015$ & 135 & 280 \\
\hline & $09 / 2015$ & 120 & 230 \\
\hline & $03 / 2016$ & 115 & 180 \\
\hline & $11 / 2016$ & 125 & 200 \\
\hline & $02 / 2018$ & 130 & 200 \\
\hline & $03 / 2018$ & 160 & 250 \\
\hline & $03 / 2018$ & 130 & 230 \\
\hline
\end{tabular}

Results: Operating time (min) average resulted 97 for RAUR and 132 for OUR, while estimated blood loss $(\mathrm{ml})$ average resulted respectively 55,7 and 235,7. Data during the time were reported in Table I, while the learning curves designed from the collected data appear in Figure 1. There is a statistically significant difference $(p<0.05)$ in both parameters (Mann-Whitney $U$-test). Discussion: There are no studies in the literature that report the LC of RAUR. The analysis of our results showed in the LC of RAUR procedures a classical trend of the LC observed in other settings: an initial slow improvement followed by the steep phase (a rapid improvement) and an initial plateau, although we need further cases to confirm the successful stabilization of learning. However, it is interesting to point out how a single surgeon in training for a robotic procedure, with a previous experience of 30-50 open procedures, has reached, in thirteen procedures and without a high intensity of training (the procedures carried out in a period of two years), better parameters than the expert surgeon with OUR procedures. On the other hand, in the LC of OUR, the two parameters are already in plateau, a sign that the operator has achieved the best competence in 


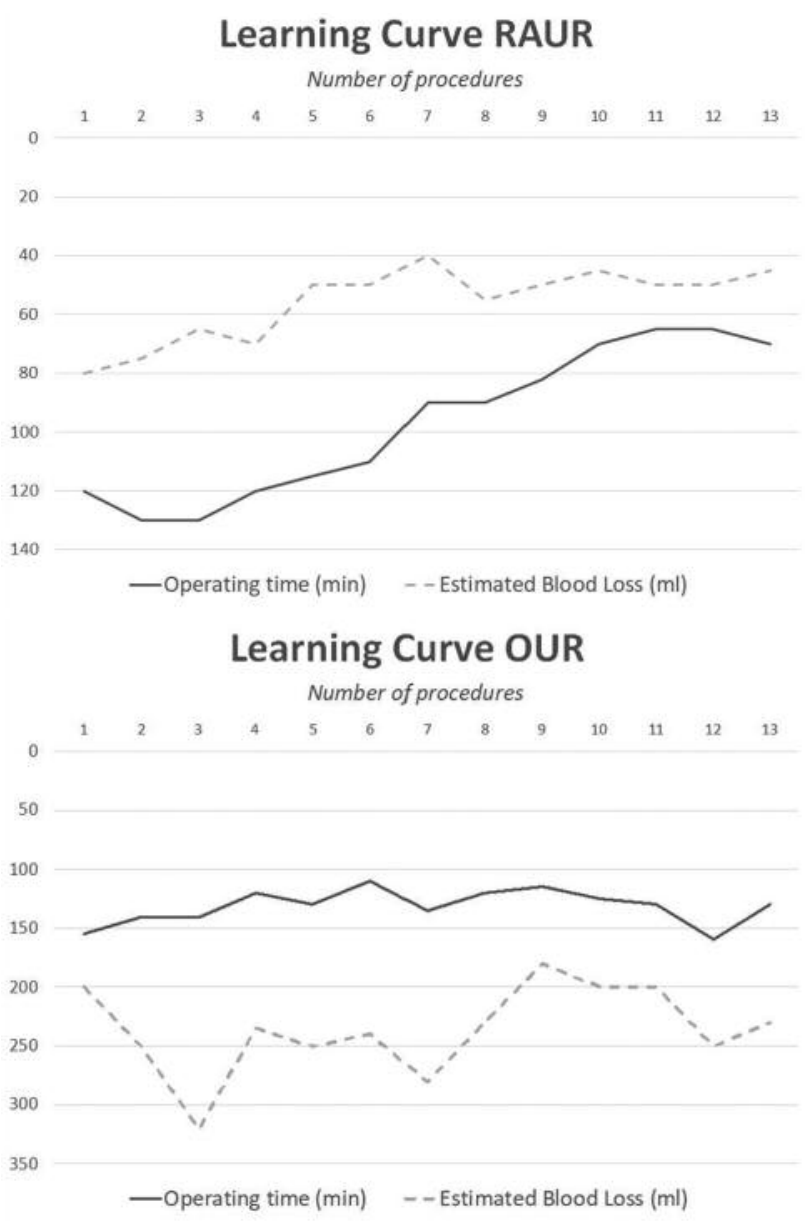

Figure 1. In the first chart related to RAUR procedure, the learning curve of single operator in training based on Operating Time ( $\mathrm{min}$ ) and Estimated Blood Loss $(\mathrm{ml})$ shows a classical trend of the LC: an initial slow improvement, the steep phase and an initial plateau. In the second chart related to OUR procedure and base on the same parameters, the learning curve of a single expert operator shows a stabilized plateau.

terms of operating time and estimated intraoperative blood loss. Unfortunately, it is not possible to compare the data in the literature to highlight any similarities or differences. However, it is possible to state that the robot is a perfect assistant for a young surgeon. A parameter to be considered in the use of the robot, which is emerging in the comparative studies of outcomes and costs between robotic and open surgery, is its utility in reducing the surgeon's learning curve: a comment to the unique RCT on the robotic prostatecomy $v s$. open prostatectomy appeared on the European Urology, and signed by the Italian Maurizio Brausi (2), claims that need to put the non-statistical difference between the two procedures into context of the operator. About this, the robotic prostatectomies were performed by a young surgeon who has as experience a number of 200 robotic prostatectomies while the open prostatectomies were carried out by an experienced surgeon, with an experience of 1500 . Conclusion: Learning-curve for RAUR has shown that thirteen procedures allow to reach an initial plateau. In the comparison studies between the robotic technique and open technique it is necessary to include, in the total economy, also the saving of money and time in terms of "training" for the young surgeons: a young urologist can reach performance levels of an experienced urologist in shorter time if supported by the robot.

1 Mazzon G, Sridhar A, Busuttil G, Thompson J, Nathan S, Briggs T, Kelly J and Shaw G: Learning curves for robotic surgery: A review of the recent literature. Curr Urol Rep 18: 89, 2017. PMID: 28942572. DOI: $10.1007 /$ s11934017-0738-z

2 Brausi M: Re: Robot-assisted laparoscopic prostatectomy versus open radical retropubic prostatectomy: Early outcomes from a randomised controlled phase 3 study. Eur Urol 72: 856-857, 2017. PMID: 28851579. DOI: $10.1016 /$ j.eururo.2017.07.029

\section{1 \\ LAPAROSCOPIC SUTURING DURING RADICAL PROSTATECTOMY WITH FLEXDEX SYSTEM: FIRST CASE IN EUROPE}

Luca De Zorzi, Antonio Amodeo and Claudia Cargne

Department of Urology, Istituto Oncologico Veneto (IOV) IRCCS, Castelfranco Veneto, Italy

Introduction: Laparoscopic radical prostatectomy is a miniinvasive technique and was introduced in the 1990s $(1,2)$. It aims to replicate the results that have been obtained by open radical retropubic prostatectomy while reducing the morbidity associated with surgery. Since its introduction, laparoscopic radical prostatectomy has undergone numerous modifications in surgical technique, including approach (transperitoneal (2) vs. extraperitoneal (3)), anterior (4) and posterior (2) dissection, ascending (5) and descending (4) dissection, and most notably, robotic-assisted. As with all new techniques, the learning curve must be surmounted to become proficient, and modifications are made to improve outcome. In specific various surgical techniques can be employed to perform the anastomosis during laparoscopic radical prostatectomy. Many surgeons prefer the robot-assisted procedure because of its ergonomic advantages, but the technology remains expensive. A key feature is the patented FlexDex "Virtual Center" ${ }^{\mathrm{TM}}$ " mechanism (Figure 1). This low-cost, purely mechanical control system reproduces the surgeon's natural hand to wrist movements at the articulating instrument jaw. Avoiding the cost and complexity 
of robotically controlled systems, the Virtual Center mechanism simply extends dynamic control and functionality to mechanical instruments. The elegant design of the Infinity Handle delivers fingertip control of the articulating jaw. A simple lever opens and closes the jaw while the rotation dial allows the surgeon to efficiently rotate the instrument. The 3Axis Wrist Gimbal, compared to standard devices, significantly reduces instrument tremor, provides improved ergonomics and expands the surgical working area inside the patient (Figure 2). It may suggest an improved solution to the treatment of anastomosis during radical prostatectomy, in a totally mechanical way and more cost effective than robotic surgery (Figure 3). Materials and Methods: Our patient was a 68-yearold man with clinically localized prostate cancer (ct1). Preoperative prostate biopsy documents a carcinoma Gleason score 7 and PSA $5.16 \mathrm{ng} / \mathrm{ml}$. We performed, for the first time in Europe, a laparoscopic extraperitoneal radical prostatectomy using, for suturing and for anastomosis, the FlexDex ${ }^{\mathrm{TM}}$ articulating needle driver, in a center without a robot available. Results: The patient underwent laparoscopic extraperitoneal radical prostatectomy using the FlexDex ${ }^{\mathrm{TM}}$ articulating needle driver. Time of operation was $115 \mathrm{~min}$. The vesicourethral anastomosis was created using a running stitch placed first at the 5 o'clock position on the posterolateral aspect of the bladder outside-in and then through the urethra at the same location inside-out. Proceeding anticlockwise, the running suture was placed 5 times more through both the bladder neck and the urethra in a similar fashion until it meets met the free end at the 5 o'clock position. A single knot was then tied outside the bladder. No complication occurred during the procedure. The pelvic drainage was placed and then removed in the first post-operative day. The patient was mobilized and ate in first post-operative day. In sixth postoperative day a cystography with anteroposterior and lateral views was performed and the patient removed the catheter. After catheter removal, the patient started a rehabilitation training with pelvic floor exercises. At 3 months postoperatively the patient has a complete urinary continence and PSA is $<0.008 \mathrm{ng} / \mathrm{ml}$. Conclusion: It is well established that the learning curve for laparoscopy is steeper than for robotic surgery and instruments like the FlexDex ${ }^{\mathrm{TM}}$ may help bridge this difference. The instrument may provide an alternative to the surgeon that is both more intuitive to use than traditional laparoscopy and more cost effective than robotic surgery.

1 Schuessler WW, Schulam PG, Clayman RV and Kavoussi LR: Laparoscopic radical prostatectomy: initial short-term experience. Urology 50: 854-857, 1997. PMID: 9426713. DOI: $10.1016 / \mathrm{S} 0090-4295(97) 00543-8$

2 Guillonneau B and Vallancien G: Laparoscopic radical prostatectomy: initial experience and preliminary assessment after 65 operations. Prostate 39: 71-75, 1999. PMID: 10221270 .

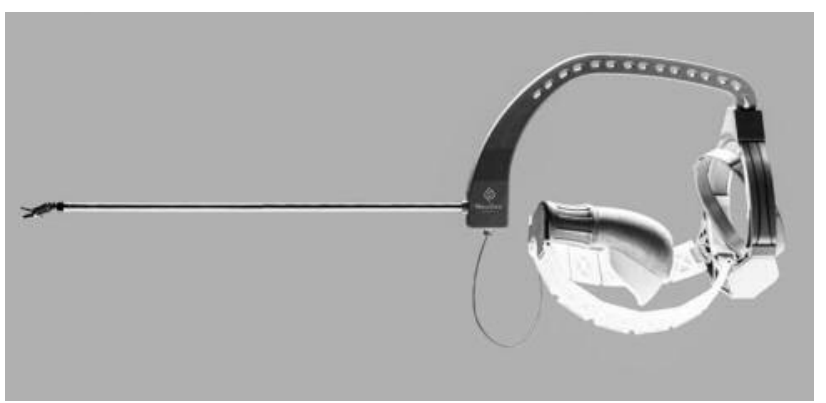

Figure 1. Laterally vision of FlexDexTM System. A simple lever opens and closes the jaw while the rotation dial allows the surgeon to rotate the instrument, and to achieve the dexterity of a robot in a mechanical device.

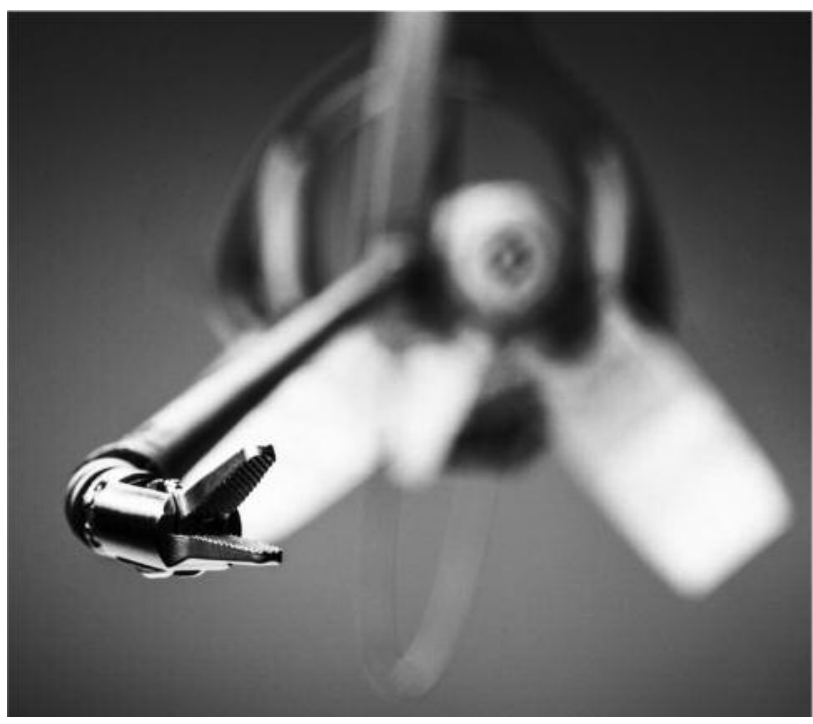

Figure 2. Zoom of the Needle-Driver of the FlexDexTM System.

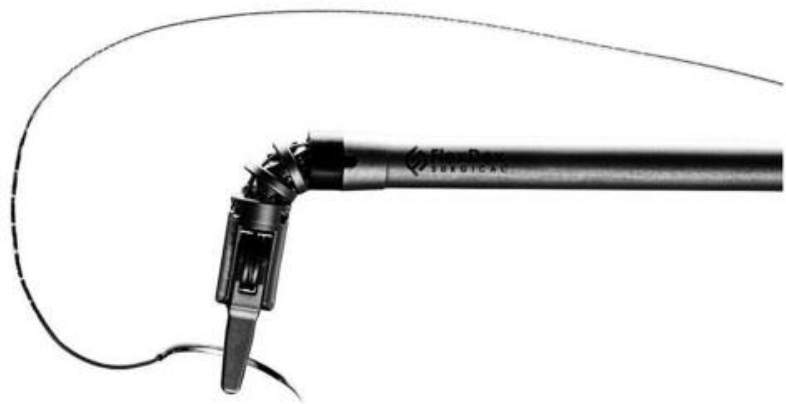

Figure 3. The 3-Axis Wrist Gimbal significantly reduces instrument tremor, provides improved ergonomics when the surgeon brings the needle and allows to perform a better suturing compared to traditional laparoscopy. 
3 Bollens R, Vanden Bossche M, Roumeguere T, Damoun A, Ekane S, Hoffmann P, Zlotta AR and Schulman CC: Extraperitoneal laparoscopic radical prostatectomy. Results after cases. Eur Urol 40: 65-69, 2001. PMID: 11528178. DOI: $10.1159 / 000049750$

4 Rhee HK, Triaca V, Sorcini A and Tuerk IA: Transperitoneal laparoscopic radical prostatectomy: descending technique. J Endourol 18: 601-604, 2004. PMID: 15597644. DOI: 10.1089/end.2004.18.601

5 Rassweiler J, Marrero R, Hammady A, Erdogru T, Teber D and Frede T: Transperitoneal laparoscopic radical prostatectomy: ascending technique. J Endourol 18: 593-599, 2004. PMID: 15597643. DOI: 10.1089/end.2004.18.593

\section{2 \\ ROLE OF ${ }^{68}$ GA-PSMA-PET/CT IN THE MANAGEMENT OF BIOCHEMICAL RECURRENCE AFTER RADICAL PROSTATECTOMY: A RETROSPECTIVE ANALYSIS}

Luca Frassinelli $^{1}$, Ercole Mazzeo ${ }^{1}$, Bianca Lanfranchi ${ }^{1}$, Laura Rubino1, Giovanni Aluisio ${ }^{1}$, Napoleone Prandini ${ }^{2}$, Marco Vernaleone ${ }^{1}$, Maura Scarlattei ${ }^{3}$, Livia Ruffini $^{3}$, Frank Lohr ${ }^{1}$ and Alessio Bruni ${ }^{1}$

${ }^{1}$ Radiotherapy Unit, University Hospital

"Policlinico of Modena", Modena, Italy;

${ }^{2}$ Nuclear Medicin Unit, University Hospital

"Policlinico of Modena", Modena, Italy;

${ }^{3}$ Nuclear Medicin Unit, University Hospital

of Parma, Parma, Italy

Aim: Recurrence after primary treatment of prostate cancer is one of the major challenges in uro-oncology. Biochemical recurrence occurs in up to one third of the patients (pts) undergoing radical prostatectomy (1). Over the last decade prostate-specific membrane antigen (PSMA) positron emission tomography (PET) have developed as better tools for staging than conventional imaging (2) (3). Our aim was to retrospectively evaluate how 68Ga PSMA-11 PET/CT (GaPET) could change the management of pts affected by biochemical recurrence after radical prostatectomy for prostate cancer allowing dose escalation in local treatment or metastasis directed therapy such as stereotactic body radiotherapy in oligometastatic patients. Materials and Methods: Between April 2017 and August 201830 consecutive pts who had undergone radical prostatectomy were submitted to GaPET for biochemical relapse or persistence of PSA increase at first control after surgery. Pathological T stage was pT2a in 1pt, pT2b-c in 10 pts and pT3 in 19 pts; positive margin status was found in 19 pts, while pelvic limphadenectomy was done in 21 pts, 3 of whom had positive node. Stratification for ISUP WHO 2014 group was as follows: 8 pts group 1, 3 group 2, 9 group 3, 6 group 4 and 4 group 5 . Five of 30 pts $(17 \%)$ had also undergone adjuvant radiotherapy on prostatic bed due to clinical stage, positive margin status or persistent PSA value after surgery. At the moment of restaging the median age was 68 years (range $=43-83$ years), the median PSA value was $0.77 \mathrm{ng} / \mathrm{ml}$ (range $=0.09-12.92$ ) and the median PSA doubling time was 0,47 years (range $=0.05-2.76$ ). Before being submitted to GaPET 9 patients were staged by conventional imaging (Abdominal CT Scan and/or Bone scintigraphy), 6 patients had also a pelvic MRI and 8 patients a Choline PET-CTscan. For each pt we established the therapeutic approach we would have proposed before being submitted to GaPET. When referred to our Unit 6 pts were initially considered candidates for continuing on regular follow-up, 18 for undergoing pelvic radiotherapy, 4 for radiotherapy with concomitant androgen deprivation therapy (ADT) and 2 pts for receiving only ADT. We decided to analyze changes in treatment assignment to these pts after having received the results of GaPET. Results: The results of GaPET showed one or more uptakes suspected for localization of PCa in 11/30pts (37\%). In two pts a positive uptake was found in the prostatic bed, in 5 in pelvic nodes, while 2 pts had bone uptakes and finally two patients had 2 simultaneous uptakes, both in pelvic-nodes and bone. Analyzing PSA level pre GaPET we found that 2 of 10 pts with PSA value less than $0.5 \mathrm{ng} / \mathrm{ml}$ had pathological uptake (one node and one bone) with a crude detection rate of $20 \%$. Conversely 9 of 20 pts with PSA value equal to or more than $0.5 \mathrm{ng} / \mathrm{ml}$ had bone and/or nodal uptake with a crude detection rate of $45 \%$. For 10 pts $(36.7 \%)$ who underwent GaPET restaging, the initially suggested therapeutic approach was changed. Three pts moved from no treatment to Radiotherapy. Seven pts, previously candidates for pelvic RT, where then submitted to receive a boost on positive nodes, while one other was submitted to exclusive Stereotactic Body radiotherapy; finally, 4 patients switched directly to palliative ADT. Comparing previous restaging ${ }^{18} \mathrm{~F}$-Choline PET/CT and GaPET, 4 of 7 pts with negative Choline staging had pathological uptake with GaPET. Conclusion: Restaging with GaPET seems to have a significant impact on management and decision-making for pts with Biochemical recurrence from Prostate cancer after primary local treatment. PSMA PET seems to provide better systemic staging of disease even for lower PSA levels causing changes in the therapeutic approach in at least one third of pts. Further larger and prospective clinical trials are needed to better understand the real impact of this new radiotracer in pts with biochemical recurrence from prostate cancer.

1 Steuber T, Jilg C, Tennstedt P, De Bruycker A, Tilki D, Decaestecker K, Zilli T, Jereczek-Fossa BA, Wetterauer U, Grosu AL, Schultze-Seemann W, Heinzer H, Graefen M, 
Morlacco A, Karnes RJ and Ost P: Standard of care versus metastases-directed therapy for pet-detected nodal oligorecurrent prostate cancer following multimodality treatment: A multi-institutional case-control study. Eur Urol Focus pii: S2405-4569(18)30077-4. PMID: 29530632. DOI: 10.1016/j.euf.2018.02.015

2 De Bari B, Mazzola R, Aiello D, Fersino S, Gregucci F, Alongi P, Nicodemo M, Cavalleri S, Salgarello M and Alongi F: Could 68-Ga PSMA PET/CT become a new tool in the decision-making strategy of prostate cancer patients with biochemical recurrence of PSA after radical prostatectomy? A preliminary, monocentric series. Radiol Med 123:719-725, 2018. PMID: 29687208. DOI: 10.1007/s11547-018-0890-7

3 Calais J, Fendler WP, Eiber M, Gartmann J, Chu FI, Nickols NG, Reiter RE, Rettig MB, Marks LS, Ahlering TE, Huynh LM, Slavik R, Gupta P, Quon A, Allen-Auerbach MS, Czernin $\mathrm{J}$ and Herrmann $\mathrm{K}$ : Impact of 68Ga-PSMA-11 PET/CT on the Management of Prostate Cancer Patients with Biochemical Recurrence. J Nucl Med 59:434-441. PMID: 29687208. DOI: 10.1007/s11547-018-0890-7

\section{3 \\ ASSOCIATION OF QUANTITATIVE MRI-BASED RADIOMIC FEATURES WITH PROGNOSTIC FACTORS IN PROSTATE CANCER}

Giulia Riva $^{1}$, Delia Ciardo ${ }^{1}$, Giulia Marvaso ${ }^{1}$, Sara Raimondi ${ }^{2}$, Stefania Volpe ${ }^{1}$, Francesca Botta ${ }^{3}$, Linda Bianchini ${ }^{4}$, Damaris Patricia Rojas ${ }^{1}$, Giuseppe Petralia ${ }^{5}$, Sarah Aless ${ }^{\mathrm{i} 5}$, Paola Pricolo ${ }^{5}$, Ottavio De Cobelli ${ }^{6}$, Roberto Orecchia ${ }^{7}$, Marta Cremonesi ${ }^{8}$, Massimo Bellomi ${ }^{5}$ and Barbara Alicja Jereczek-Fossa ${ }^{1}$

${ }^{1}$ Division of Radiotherapy, IEO - European Institute of Oncology IRCCS, Milan, Italy;

${ }^{2}$ Division of Epidemiology and Biostatistics, IEO -

European Institute of Oncology IRCCS, Milan, Italy;

${ }^{3}$ Unit of Medical Physics, IEO - European

Institute of Oncology IRCCS, Milan, Italy;

${ }^{4}$ Department of Physics, University of Milan, Milan, Italy;

${ }^{5}$ Department of Radiology, IEO - European

Institute of Oncology IRCCS, Milan, Italy;

${ }^{6}$ Department of Urology, IEO - European

Institute of Oncology IRCCS, Milan, Italy;

${ }^{7}$ Scientific Direction, IEO - European

Institute of Oncology IRCCS, Milan, Italy;

${ }^{8}$ Radiation Research Unit, IEO - European

Institute of Oncology IRCCS, Milan, Italy

Aim: To decode tumour phenotype in prostate cancer $(\mathrm{PCa})$ using a radiomic approach based on multiparametric magnetic resonance imaging (MRI). Materials and Methods: Give-me-five trial is a prospective phase II study designed for the treatment of $\mathrm{PCa}$ patients with ultrahypofractionated radiotherapy scheduled in 5 fractions with 36.25 Gy delivered to the whole prostate and a concomitant boost of $37.5 \mathrm{~Gy}$ to the dominant intraprostatic lesion (DIL) identified by multiparametric MRI. T2-weighted (T2W) MRI sequences acquired on a 1.5T Magnetom AvantoFit scanner (Siemens) with homogenous characteristics in terms of acquisition protocol were selected and the prostate gland contours were analysed. The extraction of radiomic features (shape, first-order statistics and textural features) was performed using the IBEX software after applying a 8 bit 3-sigma normalization and hierarchical clustering was applied to reduce features redundancy. We tested univariate association of each feature with the Gleason score (GS, 3+3 vs. 3+4 vs. 4+3), extracapsular extension (ECE, 1/2 vs. 2 vs. 3) score, Prostate Imaging - Reporting and Data System (PIRADS, 2/3 vs. 4 vs. 5) score and risk class (intermediate vs. low) by the Kruskal-Wallis test and selected the feature with the lowest $p$-value in each cluster. We calculated both original $p$-values and False Discovery Rate (FDR) corrected $p$ values to adjust for multiple testing. We performed multinomial cumulative logistic regression models and reported the c-statistic for model discrimination (results not shown). Statistical analysis was performed with SAS/STAT ${ }^{\circledR}$ software. Results: Of the 65 prospectively enrolled patients, 49 T2W-MRI sequences fulfilled the inclusion criteria. Baseline characteristics of the study population are reported in Table. For each patient, 636 radiomic features were identified and then grouped in 10 clusters to reduce dimensionality. At univariate analysis, higher GS was associated with higher values of the texture feature GLRLM25_0LongRunLowGrayLevelEmpha $\quad(p=0.005$, FDR adjusted $p=0.05)$ and lower values of the shape feature Compactness $2(p=0.02$, FDR adjusted $p=0.08)$. Higher ECE score was associated with lower values of the histogram feature ID_GlobalEntropy ( $p=0.03$, FDR adjusted $p=0.10)$. Higher PIRADS score was associated with lower values of the texture feature GLCM25_45-4Entropy ( $p=0.01$, FDR adjusted $p=0.06$ ). Higher risk class was associated with higher values of the texture feature GLCM25_135-4Energy $(p=0.01$, FDR adjusted $p=0.06)$. Boxplots in Figure 1 show the distribution of these radiomic features according to the prognostic factors. Conclusion: MRI-based radiomics in PCa for the prediction of tumour phenotype is a feasible and promising approach. It might lead to a semi-automated definition of tumour characteristics and thus reduce the intra/inter-operator variability in the radiologic image interpretation. We plan to increase the dataset dimensionality in order to strengthen the statistical power and to validate results. 

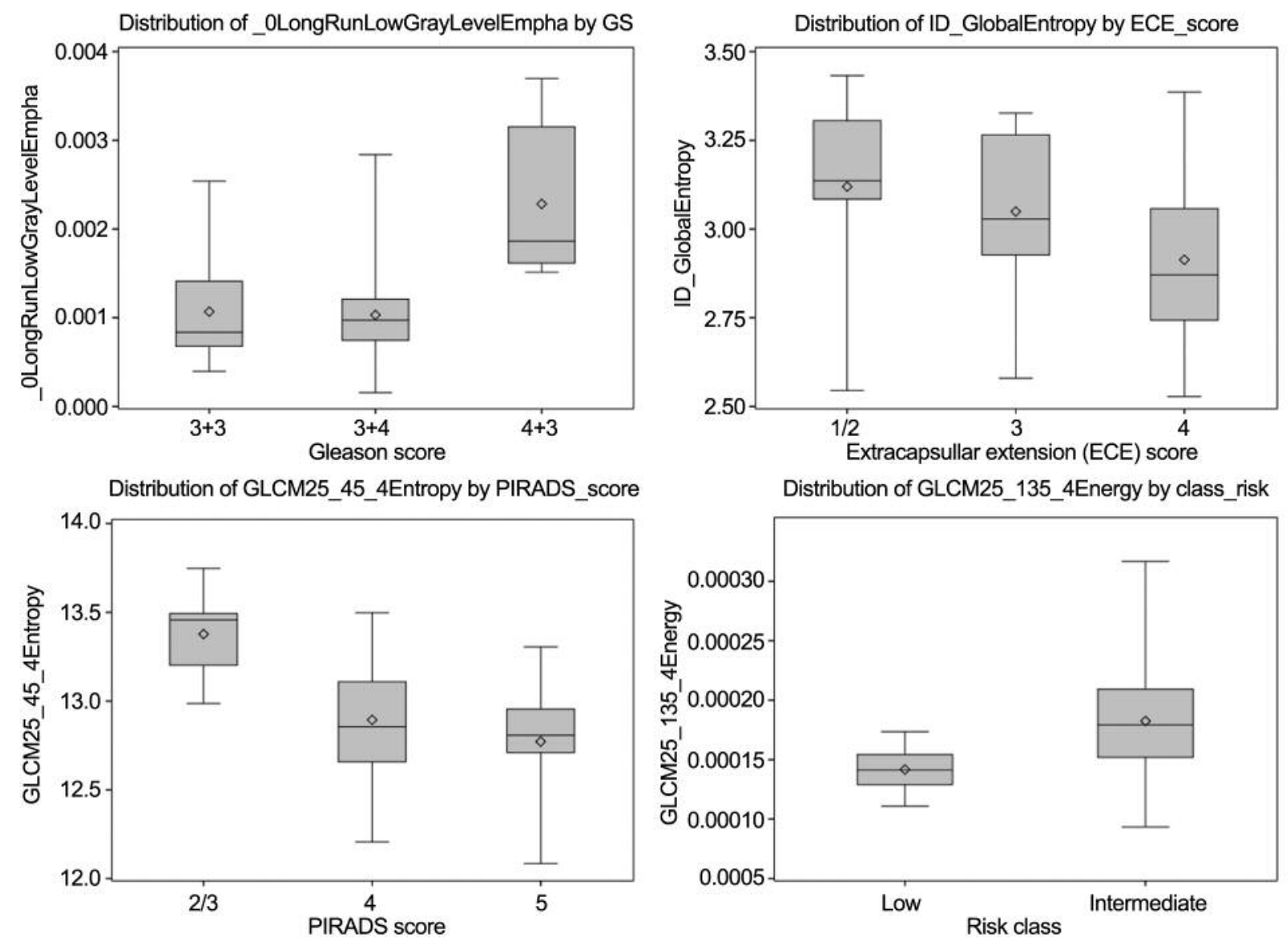

Figure 1. Boxplot representing the distribution of radiomic features according to prognostic factors.

\section{4}

\section{LOCALIZED LOW-RISK PROSTATE CANCER AND CANCER-SPECIFIC ANXIETY: RESULTS OF THE START STUDY GROUP}

$\underline{\text { Stefano De Luca }}^{1}$, Diletta Garrou$^{1}$, Oscar Bertetto ${ }^{2}$, Giovannino Ciccone ${ }^{3}$, Rosalba Rosato ${ }^{3}$, Claudia Galassi ${ }^{3}$, Enrico Bollito ${ }^{4}$, Paolo Gontero ${ }^{2}$, Alessandro Volpe ${ }^{5}$, Giovanni Cattaneo ${ }^{1}$, Daniele Amparore ${ }^{1}$, Enrico Checcucci ${ }^{1}$, Matteo Manfredi ${ }^{1}$, Francesca Ragni ${ }^{1}$, Gabriele Volpi ${ }^{1}$, Daniele Tota ${ }^{4}$, Sabrina De Cillis ${ }^{1}$,

Cristian Fiori ${ }^{1}$ and Francesco Porpiglia ${ }^{1}$

${ }^{1}$ Department of Urology, AOU

San Luigi Gonzaga, Orbassano, Italy;

${ }^{2}$ Department of Oncology, Molinette San Giovanni Battista Hospital, AOU Città della Salute, Turin, Italy;

${ }^{3}$ SSD Clinical Epidemiology, AOU Città della Salute, Turin, Italy;

${ }^{4}$ Department of Pathology, AOU

San Luigi Gonzaga, Orbassano, Italy;

${ }^{5}$ Department of Urology, AOU Maggiore

della Carità, Novara, Italy
Introduction: Active surveillance (AS) is the preferred management of low-risk prostate cancer (LRPCa). "START" study is a multi-center prospective observational study that collects data of patients affected by LRPCa, whatever is the chosen treatment (AS, radical prostatectomy [RP] or radiotherapy $[\mathrm{RT}]$ ). More specifically, information on the patient's quality of life are collected, through simple periodically questionnaires at several timepoints. The study is reserved to patients living in Piedmont and Valle d'Aosta. START study involves about 3,000 patients. Cancer-specific anxiety during active surveillance could play a significant role in influencing long-term adherence. The objective of our study was to estimate the risk of cancer-specific anxiety among time in men who choose AS instead of radical treatment (radical prostatectomy or radiotherapy). Furthermore, we evaluated if anxiety levels changed after 6 months of follow-up. Materials and Methods: Data of patients enrolled in the START study until the 15 th of March 2018 were evaluated. Anxiety was evaluated with the HADS (Hospital Anxiety and Depression Scale) and rated from a minimum of 7 (no anxiety) to a maximum of 21 (maximum value of anxiety); a score between 8-10 indicates 


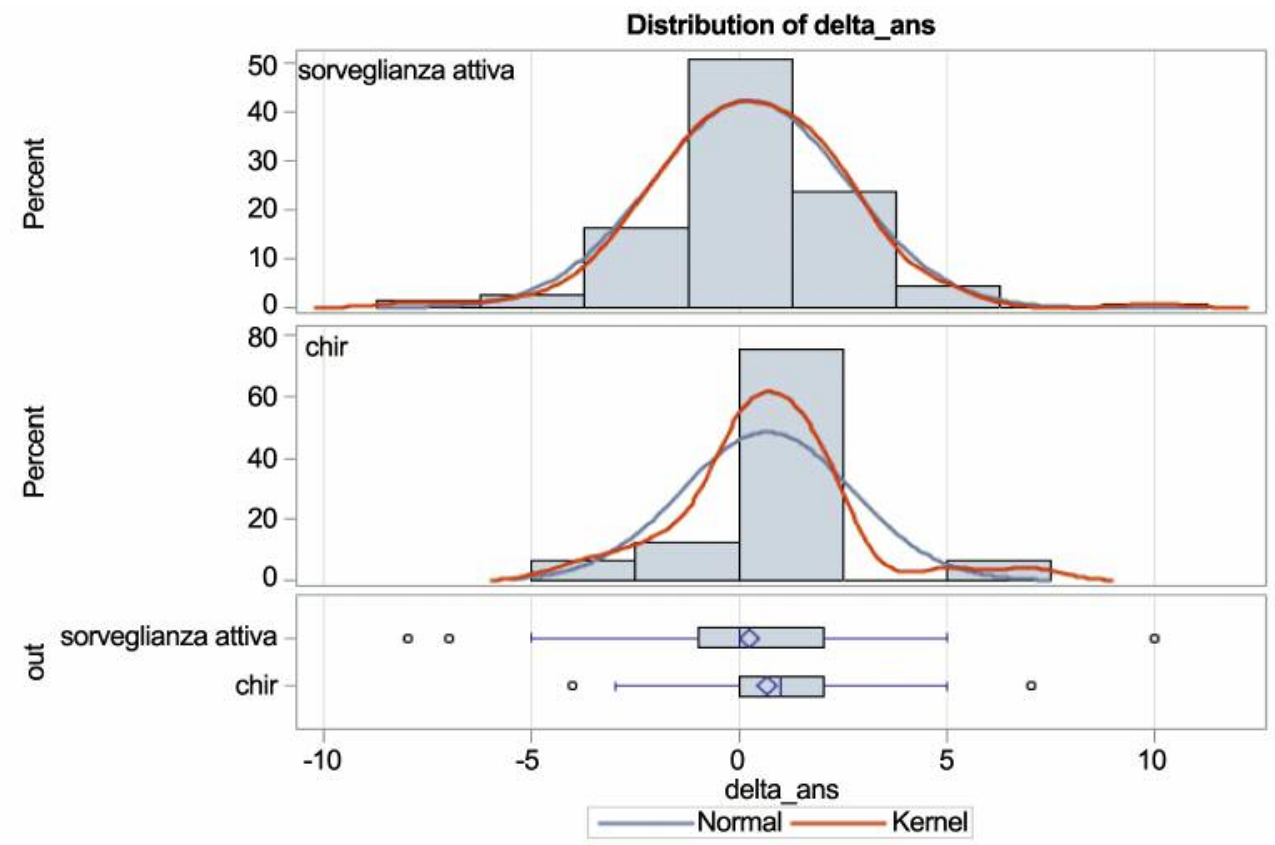

Figure 1. Distribution of anxiety in AS and RP groups.

a borderline level of anxiety, a score above 10 indicates a pathological value of anxiety. We also evaluated the difference between the 6-months anxiety score and the baseline score. $\mathrm{T}$ test and the linear regression model adjusted for age and baseline anxiety value were used for statistical analysis. Results: We evaluated 330 patients. A total of 256 patients $(77.6 \%)$ chose AS, 55 patients $(16.7 \%)$ RP and 19 patients $(6 \%)$ chose RT at baseline. The mean age was 69.7 years $(+7.0)$ in patients who chose AS, 67.5 years $(+7.4)$ in patients who underwent RP, 73.2 years $(+5.4)$ in patients who chose RT. PSA was $>7 \mathrm{ng} / \mathrm{ml}$ in $31 \%$ of patients who chose AS, in $50.9 \%$ of patients who underwent RP, in $32 \%$ on patients who chose RT. Gleason Score was $3+4$ in $12.5 \%$ of patients who chose AS, $10 \%$ of patients who underwent RP, $42 \%$ in patients who chose RT. The Charlson's score of patients who underwent RP was $>2$ in $9.1 \%$ of patients, $22 \%$ in patients who chose AS. The study showed no significant difference between the three groups in term of average baseline anxiety score (available on 310 patients): $11.7(+2.4)$ for $\mathrm{AS}, 11.5(+2.0)$ for RP and $11.4(+2.1)$ for RT. 204/310 (65.8\%) anxiety questionnaires were completed after 6 months; There was a slight average increase in anxiety in all three groups between the 6-month and baseline scores, equal to 0.2 for AS, 0.7 for RP and 1.8 for RT. The 2 most represented groups (AS and RP) were compared for subsequent statistical analyzes. Figure 1 shows the distribution of anxiety change after 6 months (T6T0) in the AS and RP groups. There is no statistical difference (0.5) in terms of anxiety between the two groups $(\mathrm{t}=1.04, p=0.30)$. The regression model, adjusted for age and baseline anxiety, did not show any difference in change between the two groups $(\mathrm{b}=0.11$, if $=0.38, p=0.77)$. Discussion and Conclusion: Only few studies have investigated how levels of prostate cancer anxiety change during the course of active surveillance or after radical treatment. The current published literature is lacking in terms of sample size and follow-up duration. Our data suggested that there is not a statistically significant difference in terms of anxiety in men with LRPCa who choose AS instead of RP and after 6 months. This is a preliminary study and other studies are necessary to assess any medium-term difference, but we think it's useful a systematic follow-up during expectant management of prostate cancer

\section{7}

\section{${ }^{68}$ GA-PSMA PET-TC-GUIDED METASTASES DIRECTED STEREOTACTIC BODY RADIOTHERAPY IN PROSTATE CANCER PATIENTS: A MONO-ISTITUTIONAL PRELIMINARY EXPERIENCE}

Michele Rigo, Rosario Mazzola, Vanessa Figlia, Luca Nicosia, Niccolo Giaj-Levra, Francesco Ricchetti, Ruggero Ruggieri and Filippo Alongi

Division of Radiation Oncology, Sacro Cuore don Calabria Cancer Care Center, Negrar, Italy 
Aim: To investigate the efficacy and toxicity of ${ }^{68} \mathrm{Ga}$-PSMA PET-CT-guided stereotactic radiotherapy (SBRT) in the treatment of oligometastatic prostate cancer. Materials and Methods: A total of 34 prostate cancer patients with biochemical relapse ( 22 castration sensitive and 12 castration resistant) were treated with Volumetric Modulated Arc Therapy and Image-Guided RT (VMAT-IGRT) on $\leq 5$ metastastatic sites detected by 68Ga PSMA PET-CT. Androgen-deprivation therapy was continued in castrationresistant (CR) patients. Results: A total of 74 metastases in 34 patients were treated with SBRT. The involved sites were pelvic lymph or paraaortic nodes $(n=53)$, bone $(n=13)$, seminal vesicles $(n=1)$, lung metastases $(n=2)$ and relapses in prostate or prostatic bed $(\mathrm{n}=5)$. The median PSA prior to RT was $0.65 \mathrm{ng} / \mathrm{mL}$ (range $=0.14-6.49 \mathrm{ng} / \mathrm{ml}$ ), the median PSA-doubling time was 5.9 months (range $=0.61-140$ ) and the median PSA post RT was $0.61 \mathrm{ng} / \mathrm{ml}$ (range $=0.02-30)$. A median dose of $35 \mathrm{~Gy}$ (range $=25-70 \mathrm{~Gy}$ ) was delivered by VMAT-IGRT in 5-10 fractions (the median BED2Gy was $144 \mathrm{~Gy}$ ). At a median follow-up of 12.6 months (range=324 months), 16 patients out of 34 patients irradiated (47\%) were in remission and 18 were in progression. In particular, 8 out of 12 castration resistant (CR) patients (67\%) and 8 out of 22 castration sensitive (CS) patients $(36 \%)$ were in progression. The actuarial 1-year LC, PFS and CSS rates were 93,47 and $100 \%$. Systemic treatment free survival was 8 months (range $=2-24$ months). No one patient experienced grade $\geq 3$ acute gastrointestinal or urinary toxicity. Conclusion: By providing optimal LC, low toxicity and a promising PFS, 68Ga PSMA PET-CT-guided metastases directed SBRT may be considered a promising treatment strategy in patients with oligometastatic prostate cancer, allowing to postpone systemic therapies.

\section{8}

\section{CAN ORAL ADMINISTRATION OF ELLAGIC ACID AND ANNONA MURICATA ENHANCE THE PROPHYLACTIC EFFICACY OF SHORT-TERM INTRAVESICAL THERAPY IN NON-MUSCLE INVASIVE BLADDER CANCER?}

Vincenzo Serretta ${ }^{1}$, Ettore De Berardinis ${ }^{2}$, Cristina Scalici Gesolfo ${ }^{1}$, Alessio Guarneri ${ }^{1}$, Calogero Guzzardo ${ }^{1}$, Sandro Billeci ${ }^{1}$, Alchiede Simonato ${ }^{1}$, Gian Maria Busetto ${ }^{2}$, Francesco Del Giudice ${ }^{2}$, Chiara Sanfilippo ${ }^{3}$ and Members of GSTU Foundation ${ }^{4}$

${ }^{1}$ Section of Urology, Department of Surgical, Oncological and Oral Sciences, University of Palermo, Palermo, Italy; ${ }^{2}$ Section of Urology, Sapienza University, Rome, Italy;

${ }^{3}$ Statistics, GSTU Foundation, Palermo, Italy;

${ }^{4}$ Gruppo Studi Tumori Urologici (GSTU)

Foundation, Palermo, Italy

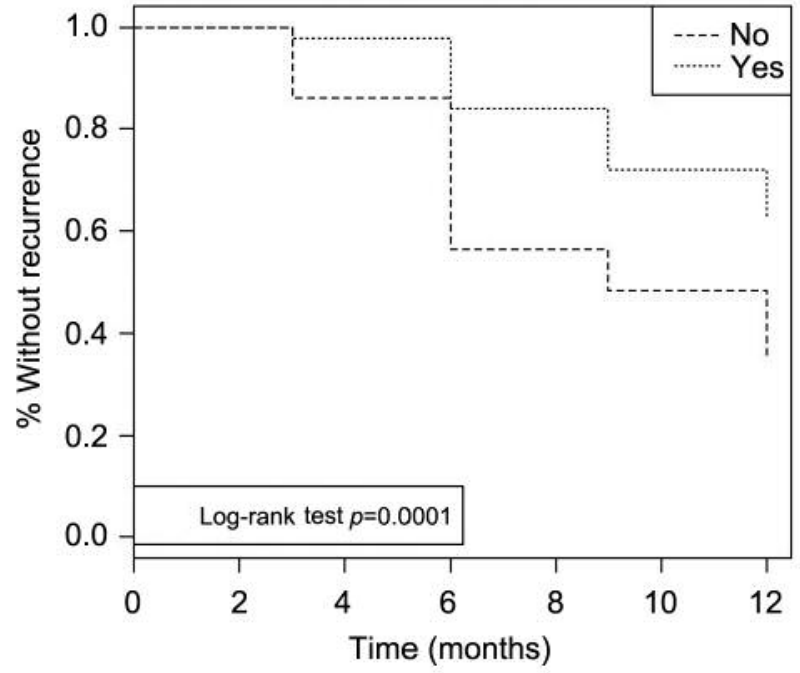

Figure 1. RFS according to AE+AM treatment.

Introduction: Ellagic acid (EA) is a polyphenolic naturally present in pomegranates, berries, grapes, green tea and nuts. The antitumor effect of EA has been reported in vitro against different human bladder cancer cell lines. Preliminary in vitro studies indicate that EA exerts anti-proliferative effects not only as a single agent, but also enhancing the antitumor activity of mitomycin C. Moreover, it could down-regulate the expression of programmed cell death ligand 1 (PD-L1), an immune checkpoint involved in immune escape (1). Annona muricata (AM) is a small deciduous tropical evergreen fruit tree, widely distributed in tropical and subtropical regions around the world. The reported therapeutic benefits of AM against various human tumors in vitro culture and in preclinical animal models (2). We present a preliminary prospective study on the activity of a combination of EA and $\mathrm{AM}$ in short-term prevention of recurrence after an induction cycle of intravesical chemotherapy or BCG. Materials and Methods: Patients affected by non-muscle invasive bladder cancer (NMIBC) after transurethral resection and intravesical prophylaxis by an induction 6-week cycle of chemiotherapy or BCG were entered in a prospective observational nonrandomized study. After informed consent and according to patient's choice, they were subdivided into two groups in relation to the oral assumption of $100 \mathrm{mg}$ of EA plus $100 \mathrm{mg}$ of AM daily for 6 months. All patients were submitted to 3month cytology and cystoscopy. Main criteria of exclusion were low-risk, Tis and very-high risk tumors according to EAU guidelines, intravesical prophylaxis exceeding 6 weeks. The association of treatment with categorical variables was assessed using $\chi^{2}$ test. All $p$-values were two-sided, and statistical significance was defined as $p<0.05$. Statistical analyses were performed using R 3.4.2 statistical software. Results: Out of 180 entered patients, 162 (90\%) were 
Table I. Association of clinic and pathologic features of 162 patients divided in two groups according to the assumption of EA+AM.

\begin{tabular}{|c|c|c|c|c|c|}
\hline & \multicolumn{2}{|c|}{ Ellagic acid } & \multirow[b]{2}{*}{ Total } & \multirow[b]{2}{*}{$p$-Value } & \multirow[b]{2}{*}{ Pts $n^{\circ}$} \\
\hline & Yes & No & & & \\
\hline Patients, n (\%) & $90(56 \%)$ & $72(44 \%)$ & 162 & & \\
\hline Gender & & & & 0.6777 & $\mathrm{n}=159$ \\
\hline Female & $18(20 \%)$ & $12(17.4 \%)$ & $30(18.9 \%)$ & & \\
\hline Male & $72(80 \%)$ & $57(82.6 \%)$ & $129(81.1 \%)$ & & \\
\hline T-stage & & & & 0.885 & $\mathrm{n}=157$ \\
\hline $\mathrm{Ta}$ & $50(56.8 \%)$ & $40(58 \%)$ & $90(57.3 \%)$ & & \\
\hline $\mathrm{T} 1$ & $38(43.2 \%)$ & $29(42 \%)$ & $67(42.7 \%)$ & & \\
\hline Grade & & & & 0.151 & $\mathrm{n}=157$ \\
\hline LG & $18(20.5 \%)$ & $21(30.4 \%)$ & $39(24.8 \%)$ & & \\
\hline $\mathrm{HG}$ & $70(79.5 \%)$ & $48(69.6 \%)$ & $118(75.2 \%)$ & & \\
\hline Previous history & & & & 0.899 & $\mathrm{n}=161$ \\
\hline Primitive & $46(51.1 \%)$ & $37(52.1 \%)$ & $83(51.6 \%)$ & & \\
\hline Recurrent & $44(48.9 \%)$ & $34(47.9 \%)$ & $78(48.4 \%)$ & & \\
\hline Focality & & & & 0.464 & $\mathrm{n}=160$ \\
\hline Single & $54(60.7 \%)$ & $39(54.9 \%)$ & $93(58.1 \%)$ & & \\
\hline Multiple & $35(39.3 \%)$ & $32(45.1 \%)$ & $67(41.9 \%)$ & & \\
\hline Drug & & & & 0.134 & $\mathrm{n}=160$ \\
\hline $\mathrm{BCG}$ & $52(59.1 \%)$ & $34(47.2 \%)$ & $86(53.8 \%)$ & & \\
\hline Chemotherapy & $36(40.9 \%)$ & $38(52.8 \%)$ & $74(46.2 \%)$ & & \\
\hline Early instillation & & & & 0.507 & $\mathrm{n}=159$ \\
\hline Yes & $2(2.3 \%)$ & $3(4.2 \%)$ & $5(3.1 \%)$ & & \\
\hline No & $85(97.7 \%)$ & $69(95.8 \%)$ & $154(96.9 \%)$ & & \\
\hline
\end{tabular}

BCG: Bacillus Calmette-Guérin; Chemotherapy: include mitomycin C 40/40 mg/ml, Epirubicin 50- 80/50 mg/ml.

evaluable, 90 and 72 receiving or not $\mathrm{EA}+\mathrm{AM}$, respectively. No statistically significant difference emerged between the 2 groups in patients' characteristics (Table I). BCG was given in $86(53.8 \%)$ and chemotherapy in $74(46.2 \%)$ patients. Early instillation before the 6-week cycle was administered in only 5 patients $(3.1 \%)$. Only $2(2.2 \%)$ patients prematurely interrupted the assumption of EA+AM and 8 (4.9\%) were lost at follow-up before the first cystoscopy. At 3, 6 and 12 months $12(7.9 \%), 43(28.3 \%)$ and $73(48.0 \%)$ patients recurred. The recurrence free rate (RFR) at 3, 6 and 12 months in patients assuming or not EA was $96.5 \%$ versus $84.6 \% \quad(p=0.003)$, $85.4 \%$ versus $64.8 \%(p=0.005)$ and $74.2 \%$ versus $60.6 \%$ $(p=0.246)$, respectively. The recurrence free survival (RFS) at 12 months in patients assuming or not EA was $63.0 \%$ versus $34.5 \%$. $(p<0.0001)$ (Figure 1). Discussion and Conclusion: Main limits of our study were its non-randomized design, the limited number of patients and the short follow-up. The results obtained should be considered as preliminary and obtained by a pilot investigation. In our preliminary observational study EA oral assumption for 6 months seems to enhance the prophylactic efficacy of traditional intravesical treatment. The Authors wish to thank the GSTU Foundation for the editing and statistical support.
1 Ceci C, Tentori L, Atzori MG, Lacal PM, Bonanno E, Scimeca M, Cicconi R, Mattei M, de Martino MG, Vespasiani G, Miano R and Graziani G: Ellagic acid inhibits bladder cancer invasiveness and in vivo tumor growth. Nutrients 8: pii: E744, 2016. PMID: 27879653. DOI: $10.3390 / \mathrm{nu} 8110744$

2 Rady I, Bloch MB, Chamcheu RN, Banang Mbeumi S, Anwar MR, Mohamed H, Babatunde AS, Kuiate JR, Noubissi FK, El Sayed KA, Whitfield GK and Chamcheu JC: Anticancer properties of graviola (Annona muricata): a comprehensive mechanistic review. Oxid Med Cell Longev 2018: 1826170, 2018. PMID: 30151067. DOI: $10.1155 / 2018 / 1826170$

\section{0}

PATHOLOGICAL OUTCOMES IN FAVOURABLE VERSUS UNFAVOURABLE INTERMEDIATE RISK PROSTATE CANCER

\section{Giorgio Napodano $^{1}$, Tommaso Realfonso ${ }^{1}$,}

Antonio Campitelli ${ }^{1}$, Olivier Intilla ${ }^{1}$, Giovanni Molisso ${ }^{1}$, Maria Addesso $^{2}$, Raffaele Baio ${ }^{1}$ and Roberto Sanseverino ${ }^{1}$ 
Table I. Baseline characteristics of patients.

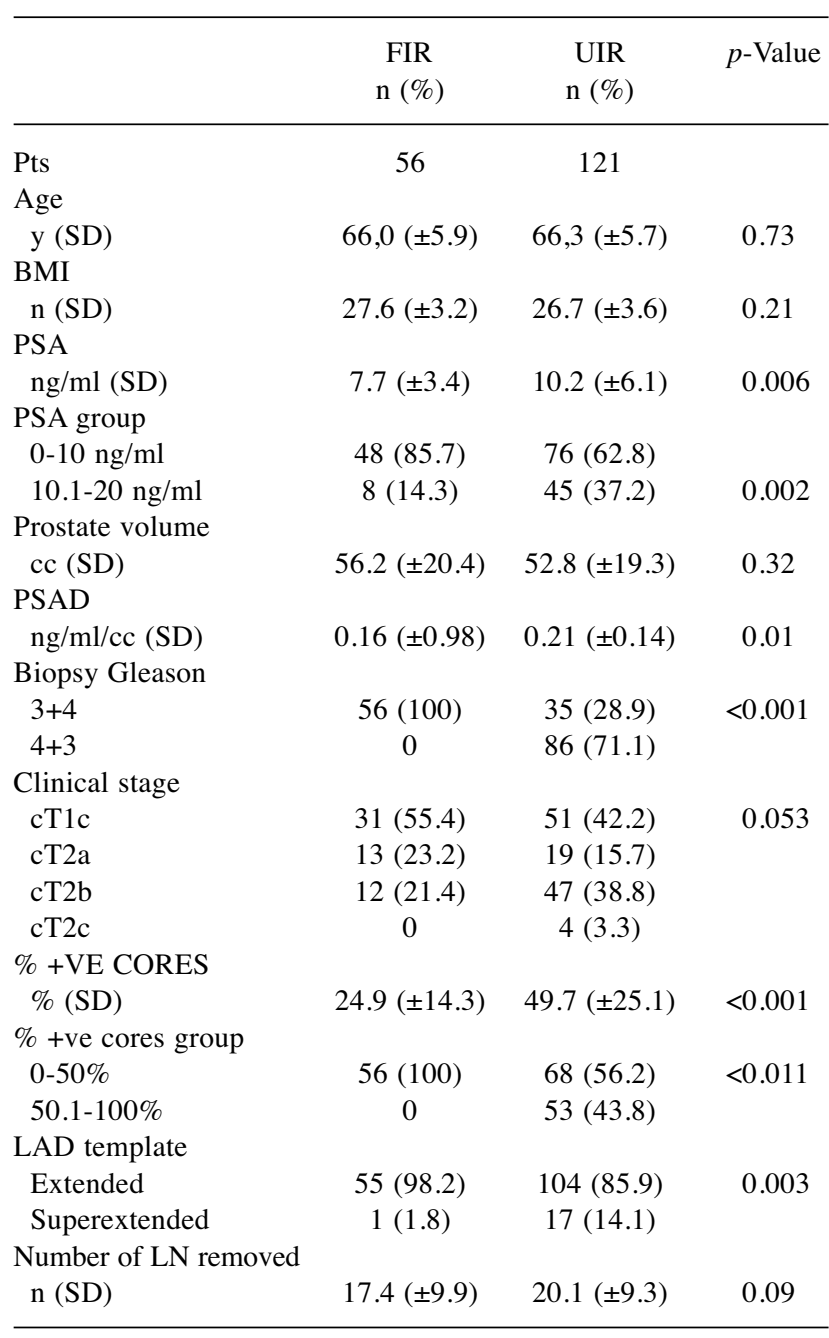

${ }^{1}$ Urology Unit, Umberto I Hospital -

ASL Salerno, Nocera Inferiore, Italy;

${ }^{2}$ Pathology Unit, Umberto I Hospital -

ASL Salerno, Nocera Inferiore, Italy

Introduction: Risk classification is the cornerstone of management of prostate cancer. The most widely used system is D'Amico stratification in low-, intermediate- and high-risk patients. However, a significant heterogeneity exists into each group. While low- and high-risk are progressively split up into two subgroups, intermediate-risk remained as miscellaneous group with a wide biological and clinical behaviour. A recent subclassification of intermediate-risk divided patients in favourable (FIR) and unfavourable (UIR) group, according to primary Gleason pattern, percentage of positive cores and number of intermediate risk factors. We compared
Table II. Pathological outcomes

\begin{tabular}{lccc}
\hline & FIR & UIR & $p$-Value \\
\hline Pathological stage & & & \\
cT2a & $11(19.6)$ & $3(2.5)$ & 0.001 \\
cT2b & $4(7.1)$ & $9(7.4)$ & \\
cT2c & $35(62.5)$ & $63(52.1)$ & \\
cT3a & $6(10.7)$ & $33(27.3)$ & \\
cT3b & 0 & $13(10.7)$ & \\
ECE & & & \\
n (\%) & $6(10.7)$ & $46(38.0)$ & $<0.001$ \\
Pathological Gleason & & & \\
3+3 & $2(3.6)$ & $3(2.5)$ & $<0.001$ \\
3+4 & $38(67.9)$ & $39(32.2)$ & \\
4+3 & $14(25.0)$ & $58(47.9)$ & \\
4+4 & $2(3.6)$ & $16(13.2)$ & \\
4+5 & 0 & $2(1.6)$ & \\
5+4 & 0 & $3(2.5)$ & \\
Upgrading & & & \\
n (\%) & $16(28.6)$ & $32(26.4)$ & 0.9 \\
Downgrading & $2(3.6)$ & $22(18.2)$ & 0.01 \\
n (\%) & & & \\
+ve surgical margins & $7(12.5)$ & $25(20.7)$ & 0.21 \\
n (\%) & & $7(5.8)$ & 0.13 \\
Nodal mets & 0 & $61(50.4)$ & $<0.001$ \\
n (\%) & $7(12.5)$ & & \\
Adverse pathology & & & \\
n (\%) & & & \\
\hline
\end{tabular}

Table III. Pathological outcomes stratified by number of intermediate risk factors.

\begin{tabular}{|c|c|c|c|}
\hline & $\begin{array}{l}\text { Group } 1 \\
\text { FIR-0 IRF }\end{array}$ & $\begin{array}{l}\text { Group } 2 \\
\text { FIR-1 IR }\end{array}$ & $\begin{array}{c}\text { Group } 3 \\
\text { UIR->1 IRF }\end{array}$ \\
\hline ECE & 5.6 & $\begin{array}{c}20 \\
v s . \text { group } 1(0.09)\end{array}$ & $\begin{array}{c}34.3 \\
v s . \text { group } 1(0.002)^{*} \\
\text { vs. group } 2(0.26)\end{array}$ \\
\hline Upgrading & 19.4 & $\begin{array}{c}45.0 \\
v s . \text { group } 1(0.04)^{*}\end{array}$ & $\begin{array}{c}42.9 \\
v s . \text { group } 1(0.03)^{*} \\
v s . \text { group } 2(0.88)\end{array}$ \\
\hline Downgrading & 7.1 & $\begin{array}{c}0 \\
v s . \text { group } 1(0.25)\end{array}$ & $\begin{array}{c}0 \\
v s . \text { group } 1(0.12) \\
v s . \text { group } 2-\end{array}$ \\
\hline +ve SM & 11.1 & $\begin{array}{c}15.0 \\
v s . \text { group } 1(0.67)\end{array}$ & $\begin{array}{c}5.7 \\
v s . \text { group } 1(0.41) \\
v s . \text { group } 2(0.25)\end{array}$ \\
\hline LNI & 0 & 0 & $\begin{array}{c}8.6 \\
v s . \text { group } 1(0.20) \\
v s . \text { group } 2(0.34)\end{array}$ \\
\hline $\begin{array}{l}\text { Adverse } \\
\text { pathology }\end{array}$ & 8.3 & $\begin{array}{c}20.0 \\
v s . \text { group } 1(0.21)\end{array}$ & $\begin{array}{c}40.0 \\
v s . \text { group } 1(0.002)^{*} \\
v s . \text { group } 2(0.13)\end{array}$ \\
\hline
\end{tabular}

pathological outcomes of FIR and UIR patients undergoing radical prostatectomy. Materials and Methods: From the database of our Institution, we identified patients with 
intermediate-risk prostate cancer udergoing laparoscopic radical prostatectomy. We defined favourable intermediate risk (FIR) as GS $3+4$ and $\leq 50 \%$ of positive biopsy cores and $\leq 1$ risk factor (cT2b-cT2c, PSA 10-20 ng/ml); the others intermediate-risk patients were classified as unfavourable intermediate risk (UIR). We correlated FIR and UIR groups with upstaging ( $\geq$ pT3) (ECE), upgrading (UG), downgrading (DG), positive surgical margins (PSM), lymphonode metastases (LNI) and adverse pathological outcomes (nonorgan confined disease or $>\mathrm{GS} 4+3$ or $\mathrm{pN} 1$ ) (AP). We have also stratified patients according to number of unfavourable intermediate risk factors (UIRF) and we have analyzed the correlation of these groups with pathological outcomes. Statistical analysis was performed using the SPSS 24 as appropriate. Results: From our database we identified 177 intermediate-risk patients. Of these $121(68.4 \%)$ patients were classified as UIR and $56(31.6 \%)$ as FIR. Baseline characteristics of the patients are described in Table I. UIR patients presented higher PSA, higher positive core percentage and more extended lymph node dissection template. UIR patients had increased risk of pathological upstaging $(p<0.001)$ and downgrading $(p<0.01)$, worse pathological grading $(p<0.001)$ and worse adverse pathological outcomes $(p<0.001)$ (Table II). There were no differences between the two groups in terms of upgrading, positive surgical margins and nodal metastases (Table II). When stratified by number of UIRF, patients with more than one UIRF had a higher risk of upstaging, upgrading and adverse pathology than patients with no UIRF (Table III). Conclusion: In our experience patients with unfavourable intermediate-risk prostate cancer have increased risk of extracapsular extension, downgrading and adverse pathological outcomes than patients with favourable intermediat-risk prostate cancer. Therefore, the number of unfavourable risk factors seems to correlate with the risk of extracapsular extension, upgrading and adverse pathological findings.

\section{1 \\ PREDICTIVE FACTORS OF PATHOLOGICAL OUTCOMES IN INTERMEDIATE-RISK PROSTATE CANCER}

Giorgio Napodano ${ }^{1}$, Tommaso Realfonso ${ }^{1}$, Antonio Campitelli ${ }^{1}$, Olivier Intilla ${ }^{1}$, Giovanni Molisso ${ }^{1}$, Raffaele Baio ${ }^{1}$, Maria Addesso ${ }^{2}$ and Roberto Sanseverino ${ }^{1}$

${ }^{1}$ Urology Unit, Umberto I Hospital ASL Salerno, Nocera Inferiore, Italy; ${ }^{2}$ Pathology Unit, Umberto I Hospital ASL Salerno, Nocera Inferiore, Italy

Introduction: Risk classification is the cornerstone of management of prostate cancer. intermediate risk remained as
Table I. Baseline characteristics of patients.

\begin{tabular}{lcc}
\hline Pts & $\mathrm{n}$ & 182 \\
\hline Age & $\mathrm{y}(\mathrm{SD})$ & $66.30( \pm 5.7)$ \\
BMI & $\mathrm{n}(\mathrm{SD})$ & $27.0( \pm 3.4)$ \\
PSA & $\mathrm{ng} / \mathrm{ml}(\mathrm{SD})$ & $9.4( \pm 5.5)$ \\
PSA group & $0-10 \mathrm{ng} / \mathrm{ml}$ & $125(68.7)$ \\
& $10.1-20 \mathrm{ng} / \mathrm{ml}$ & $57(31.3)$ \\
Prostate volume & $\mathrm{cc}(\mathrm{SD})$ & $53.9( \pm 19.5)$ \\
PSAD & $\mathrm{ng} / \mathrm{ml} / \mathrm{cc}(\mathrm{SD})$ & $0.20( \pm 0.13)$ \\
Biopsy Gleason & $3+4$ & $95(52.2)$ \\
& $4+3$ & $87(47.8)$ \\
Clinical stage & $\mathrm{cT} 1 \mathrm{c}$ & $86(47.2)$ \\
& $\mathrm{cT} 2 \mathrm{a}$ & $33(18.1)$ \\
$\%+$ VE CORES & $\mathrm{cT} 2 \mathrm{~b}$ & $63(34.7)$ \\
$\%+$ ve cores group & $\%(\mathrm{SD})$ & $41.6( \pm 24.9)$ \\
& $0-50 \%$ & $127(69.8)$ \\
LAD template & $50.1-100 \%$ & $55(30.2)$ \\
& Extended & $164(90.1)$ \\
Number of LN removed & Superextended & $18(9.9)$ \\
& $\mathrm{n}(\mathrm{SD})$ & $19.0( \pm 9.6)$ \\
\hline
\end{tabular}

Table II. Pathological outcomes.

\begin{tabular}{lcc}
\hline & & $\mathrm{n}(\%)$ \\
\hline Pathological stage & T2a & $13(7.1)$ \\
& T2b & $14(7.7)$ \\
& T2c & $102(56.0)$ \\
& T3a & $39(21.5)$ \\
& T3b & $14(7.7)$ \\
ECE & $\mathrm{n}(\%)$ & $53(29.1)$ \\
Pathological Gleason & $3+3$ & $6(3.2)$ \\
& $3+4$ & $79(43.4)$ \\
& $4+3$ & $73(40.1)$ \\
& $4+4$ & $19(10.4)$ \\
Upgrading & $4+5$ & $2(1.1)$ \\
Downgrading & $5+4$ & $3(1.6)$ \\
+ve surgical margins & $\mathrm{n}(\%)$ & $49(26.9)$ \\
Nodal mets & $\mathrm{n}(\%)$ & $25(13.7)$ \\
Adverse pathology & $\mathrm{n}(\%)$ & $34(18.7)$ \\
& $\mathrm{n}(\%)$ & $7(3.8)$ \\
& $\mathrm{n}(\%)$ & $69(37.9)$ \\
\hline
\end{tabular}

the miscellaneous group with a wide biological and clinical behaviour. The definition of predictive factors of unfavourable disease allows to substratify these patients optimizing management of intermediate risk prostate cancer. In our study we evaluated the risk of pathological unfavourable outcomes and predictive factors of adverse disease in patients with intermediate risk prostate cancer. Materials and Methods: From the database of our institution, we identified patients with intermediate-risk prostate cancer 
Table III. Multivariate analysis.

\begin{tabular}{|c|c|c|c|c|c|c|}
\hline & Upstaging & Upgrading & Downgrading & + ve SM & $\mathrm{N}+$ & Adverse pathology \\
\hline Age (y) & 0.24 & 0.11 & 0.82 & 0.24 & 0.78 & 0.58 \\
\hline BMI (n) & 0.41 & 0.39 & 0.65 & 0.60 & 0.20 & 0.37 \\
\hline PSA (ng/ml) & 0.07 & 0.12 & 0.12 & 0.06 & 0.31 & 0.27 \\
\hline \multicolumn{7}{|l|}{ PSA group } \\
\hline$\leq 10 v s .>10$ & 0.1 & 0.31 & $\begin{array}{c}0.02 \\
\text { OR } 0.15 \\
95 \% \text { CI } 0.03-0.73\end{array}$ & 0.29 & 0.81 & $\begin{array}{c}0.03 \\
\text { OR } 2.29 \\
95 \% \text { CI } 1.10-4.77\end{array}$ \\
\hline Prostate volume $(\mathrm{cc})$ & 0.06 & 0.95 & 0.07 & 0.10 & 0.33 & 0.37 \\
\hline $\operatorname{PSAD}(\mathrm{ng} / \mathrm{ml} / \mathrm{cc})$ & 0.06 & 0.72 & 0.11 & 0.07 & 0.30 & 0.61 \\
\hline Gleason score & 0.01 & 0.09 & $<0.001$ & 0.005 & 0.78 & $<0.001$ \\
\hline $3+4$ vs. $4+3$ & OR 2.43 & & OR 16.74 & OR 3.46 & & OR 4.04 \\
\hline & 95\%CI $1.2-4.9$ & & $95 \%$ CI $0.65-78.44$ & $95 \%$ CI $1.46-8.18$ & & $95 \%$ CI $2.03-8.08$ \\
\hline$\%+$ ve cores $(\%)$ & 0.33 & 0.65 & 0.43 & 0.19 & 0.39 & 0.46 \\
\hline \multicolumn{7}{|l|}{$\%+v e$ cores group } \\
\hline$\leq 50 \%$ vs. $>50 \%$ & 0.17 & 0.89 & 0.23 & 0.8 & 0.85 & 0.13 \\
\hline \multicolumn{7}{|l|}{ Clinical stage } \\
\hline $\mathrm{cT} 1 \mathrm{c} / 2 \mathrm{a} v s . \mathrm{cT} 2 \mathrm{~b}-\mathrm{c}$ & 0.14 & 0.11 & 0.41 & 0.3 & 0.49 & 0.16 \\
\hline LAD template & 0.19 & 0.54 & 0.55 & 0.16 & 0.96 & 0.79 \\
\hline $\mathrm{N}^{\circ}$ nodes removed & 0.49 & 0.56 & 0.42 & 0.19 & 0.85 & 0.27 \\
\hline
\end{tabular}

(GS 7, cT1c-T2b, PSA <20 ng/ml) undergoing laparoscopic radical prostatectomy. Data on age, BMI, PSA, PSAD, positive cores percentage, clinical stage, Gleason score, lymphadenectomy template, prostate volume, number of removed nodes were available. We correlated these variables with upstaging ( $\geq \mathrm{pT} 3$ ), upgrading, positive surgical margins (SM), lymphonode metastases (LNI) and adverse pathological outcomes (AP) (non-organ confined disease or $>$ GS4+3 or $\mathrm{pN} 1)$. Statistical analysis was performed using the SPSS $24^{\mathrm{TM}}$ as appropriate. Results: We identified 182 intermediate-risk patients. Baseline characteristics of the patients are reported in Table I. Extracapsular extension (ECE) occurred in $29.1 \%$ of the patients, upgrading in $26.9 \%$; surgical margins were positive in $18.7 \%$ and nodal metastases was found in $3.8 \%$. More than one third of patients $(37.9 \%)$ presented adverse pathological disease (Table II). At multivariate analysis only PSA and biopsy Gleason score were found to be predictive factors of pathological outcomes (Table III). PSA was associated with downgrading ( $p=0.02$; OR 0.15$)$ and adverse pathological disease $(p=0.03$; OR 2.29), while Gleason score was correlated with downgrading $(p<0.001$; OR 16.7), adverse pathological outcomes $(p<0.001$; OR 4.0), upstaging $(p=0.01$; OR 2.43) and positive surgical margins $(p=0.005$; OR 3.46). Conclusion: In our experience in patients with intermediate-risk prostate cancer, adverse pathological outcomes occurred in $38 \%$ of the patients. PSA and Gleason scores were predictive factors of downgrading, adverse pathological outcomes, upstaging and positive surgical margins.

\section{3}

\section{PREDICTIVE FACTORS OF NODAL METASTASIS IN PATIENTS WITH ORGAN-CONFINED PROSTATE CANCER}

$\underline{\text { Roberto Sanseverino }}^{1}$, Antonio Campitelli ${ }^{1}$, Tommaso Realfonso ${ }^{1}$, Giovanni Molisso ${ }^{1}$, Alfonso Baio ${ }^{1}$, Olivier Intilla ${ }^{1}$, Raffaele Baio ${ }^{1}$, Maria Addesso ${ }^{2}$ and Giorgio Napodano ${ }^{1}$

${ }^{1}$ Urology Unit, Umberto I Hospital ASL Salerno, Nocera Inferiore, Italy; ${ }^{2}$ Pathology Unit, Umberto I Hospital ASL Salerno, Nocera Inferiore, Italy

Aim: To evaluate predictive factors of nodal metastasis in patients affected by organ confined-prostate cancer (PCa) who underwent laparoscopic radical prostatectomy (LPR). Materials and Methods: From the database of our Institution, we identified patients who had undergone a laparoscopic pelvic lymphadenectomy (LAD) and radical prostatectomy; data on age, BMI, PSA, PSAD, positive cores percentage, clinical stage, Gleason score, lymphadenectomy template, prostate volume, number of removed nodes were available. We correlated these variables with pathological node metastasis by logistic regression analysis (SPSS 24 software). Results: Data on 390 patients were analyzed. Baseline characteristics are reported in Table I. On univariate analysis, age. PSA, PSAD, clinical stage, biopsy Gleason score, positive cores percentage and risk group were associated with 
Table I. Baseline characteristics.

\begin{tabular}{|c|c|c|c|c|}
\hline & All pts & No & $\mathrm{N} 1$ & $p$-Value \\
\hline Pts & 390 & 372 & 18 & \\
\hline \multicolumn{5}{|l|}{ Age } \\
\hline $\mathrm{y}$ & & $65.8 \pm 10.0$ & $60.0 \pm 28.3$ & 0.03 \\
\hline \multicolumn{5}{|l|}{ BMI } \\
\hline $\mathrm{n}$ & & $26.9 \pm 3.1$ & $28.0 \pm 3.5$ & 0.24 \\
\hline \multicolumn{5}{|l|}{ PSA } \\
\hline $\mathrm{ng} / \mathrm{ml}$ & & $9.2 \pm 5.3$ & $14.4 \pm 12.3$ & 0.001 \\
\hline \multicolumn{5}{|l|}{ Prostate volume } \\
\hline $\mathrm{cc}$ & & $54.4 \pm 20.4$ & $58.2 \pm 28.0$ & 0.49 \\
\hline \multicolumn{5}{|l|}{ PSAD } \\
\hline $\mathrm{ng} / \mathrm{ml} / \mathrm{cc}$ & & $0.19 \pm 0.13$ & $0.34 \pm 0.23$ & $<0.001$ \\
\hline \multicolumn{5}{|l|}{ C Stage } \\
\hline $\mathrm{T} 1 \mathrm{c}$ & 55.0 & 56.0 & 35.3 & 0.01 \\
\hline $\mathrm{T} 2$ & 43.9 & 43.6 & 53.0 & \\
\hline $\mathrm{T} 3$ & 1.1 & 0.4 & 11.7 & \\
\hline \multicolumn{5}{|l|}{ Biopsy gleason } \\
\hline $3+3$ & 35.0 & 36.3 & 5.9 & 0.01 \\
\hline $3+4$ & 24.3 & 24.4 & 23.5 & \\
\hline $4+3$ & 22.3 & 22.2 & 23.5 & \\
\hline$>7$ & 18.4 & 17.1 & 48.1 & \\
\hline \multicolumn{5}{|l|}{$\begin{array}{l}\text { Positive core } \\
\text { percentage }\end{array}$} \\
\hline $\mathrm{n}$ & $38.6 \pm 24.0$ & $58.3 \pm 27.3$ & 0.001 & \\
\hline \multicolumn{5}{|l|}{ Risk group } \\
\hline Low & $22.6(88)$ & $23.7(88)$ & 0 & 0.01 \\
\hline Intermediate & $51.0(199)$ & $51.3(191)$ & $44.4(8)$ & \\
\hline High & $26.4(103)$ & $25.0(93)$ & $55.6(10)$ & \\
\hline
\end{tabular}

Table II. Surgical and pathological outcomes.

\begin{tabular}{lcccc}
\hline & All pts & N0 & N1 & $p$-Value \\
\hline & 390 & 372 & 18 & \\
Path. stage & & & & \\
pT2 & 72.0 & 74.9 & 11.1 & $<0.001$ \\
pT3a & 17.7 & 17.5 & 22.2 & \\
pT3b & 9.5 & 6.7 & 66.7 & \\
pT4 & 0.8 & 0.8 & 0 & \\
Pathol. gleason & & & & \\
3+3 & 12.3 & 12.9 & 0 & 0.02 \\
3+4 & 32.3 & 33.6 & 5.6 & \\
$4+3$ & 36.4 & 35.8 & 50.0 & \\
$\quad 7$ & 18.9 & 17.7 & 44.4 & \\
Positive SM & & & & \\
$\quad \%$ & 16.9 & 14.5 & 66.7 & $<0.001$ \\
LAD template & & & & \\
$\quad$ Extended & 85.1 & 87.6 & 33.3 & $<0.001$ \\
$\quad$ Superextended & 14.9 & 12.4 & 66.7 & \\
Number nodes removed & & & & \\
$\quad \mathrm{n}$ & & $171.4 \pm 10.1$ & $26.6 \pm 10.5$ & $<0.001$ \\
\hline
\end{tabular}

Table III. Multivariate analysis.

\begin{tabular}{lccc}
\hline & OR & $p$-Value & CI (95\%) \\
\hline LAD template & 0.13 & 0.01 & $0.026-0.611$ \\
\hline
\end{tabular}

pN1. Surgical and pathological outcomes are reported in Table II. At univariate analysis, pathological stage, Gleason score, positive surgical margins, LAD template (obturator and external $v s$. obturator, external hypogastric and common) and number of removed nodes correlated with $\mathrm{pN} 1$. At multivariate analysis, superextended lymphadenectomy is associated with nodal metastasis (Table III). Conclusion: In our retrospective analysis, nodal metastases occurred in $4.6 \%$ of the patients. Superextended lymphadenectomy is a predictive factor of nodal metastasis.

35

\section{COMPARISON OF TWO NEWLY-DEVELOPED BLADDER CANCER TESTS IN THE FOLLOW-UP OF PATIENTS WITH NON-MUSCLE INVASIVE BLADDER CANCER (NMIBC): PRELIMINARY RESULTS}

Carolina D' elia $^{1}$, Emanuela Trenti ${ }^{1}$, Alexander Pycha ${ }^{2}$, Christine $\mathrm{Mian}^{3}$, Christine Schwienbacher ${ }^{3}$, Esther Hanspeter ${ }^{3}$ and Armin Pycha ${ }^{1}$

${ }^{1}$ Department of Urology, Central Hospital of Bolzano, Bolzano, Italy; ${ }^{2}$ Department of Urology, Luzerner Kantonsspital, Luzern, Switzerland; ${ }^{3}$ Department of Pathology, Central Hospital of Bolzano, Bolzano, Italy

Introduction: Cystoscopy is the most efficient method currently available for the diagnosis of primary or recurrent bladder cancer (BC), but it is invasive and causes a significant discomfort to the patient. Furthermore, flat tumors or carcinoma in situ may be difficult to detect. Urinary cytology is not invasive and very effective in diagnosing high-grade lesions, but it has a low sensitivity in low-grade tumors, which are the most common types of BC. The limitations of cytology and cystoscopy both for primary diagnosis and monitoring of patients after BC was removed, and led to development of new urine tests for the early detection of BC. The aim of this study was to compare the diagnostic value of 2 newly-developed urine tests, the mRNA-based Xpert BC Monitor and the DNA methylationbased Bladder Epicheck in patients under follow-up after 
TUR. Patients and Methods: A total of 58 patients (mean age 77 years, range 51-90 years) under follow-up for NMIBC were studied prospectively. Samples were analyzed with the Bladder Epicheck Test, the Xpert BC Monitor and voided urinary cytology. Subsequently to urine collection, the patient underwent cystoscopy and if cystoscopically positive, a TUR-B. Cytologies were evaluated according to the Paris System of reporting cytology. For the Bladder Epicheck Test a software calculates the EpiScore, a number between 0 and 100 representing the overall methylation level of the sample. If the EpiScore is equal or above 60 it is considered positive. The results of the Xpert BC Monitor are interpreted by the GeneXpert ${ }^{\circledR}$ Instrument System and given as LDA totals and Analyte Results on the Test Report. A cut-off is set at a LDA of $>0.5$. Sensitivity, specificity, PPV and NPV of Bladder Epicheck, Xpert BC Monitor and cytology were calculated using cystoscopy/histology as gold standard. Results: A total of 5 out of 58 patients $(8.6 \%)$ had to be excluded due to insufficient DNA in the Bladder Epicheck Test. A total of 13 out of 53 remaining patients had histologically-verified BC of the bladder. A total of $40 / 53$ patients were negative cystoscopically and/or histologically. Of the 13 patients with BC 8 (61.5\%) were found positive for Bladder Epicheck and Xpert BC Monitor and $4(30.7 \%)$ for cytology. The sensitivity of both tests increased from $42.8 \%$ for low-grade (LG) to $83.3 \%$ in highgrade (HG) tumours. Specificity was $77.5 \%$ (31/40) for Bladder Epicheck, 65\% (26/40) for Xpert BC Monitor and $97.5 \%$ (39/40) for voided urinary cytology. Conclusion: The Bladder Epicheck is equal to the Xpert BC Monitor in sensitivity but superior in specificity. None of both of the tests was able to reach the high specificity of cytology. Xpert BC Monitor is easy and fast to perform while the Bladder Epicheck requires dedicated technicians an $\mathrm{d}$ is more time consuming. Both tests could be, however, of interest as an additional tool in the follow-up of patients with NMIBC, reducing the number of cystoscopies.

\section{6 \\ GIVE-ME-FIVE TRIAL: TOXICITY \\ EVALUATION IN ULTRA-HYPOFRACTIONATED PROSTATE CANCER RADIOTHERAPY}

$\underline{\text { Stefania Volpe }}^{1}$, Delia Ciardo ${ }^{1}$, Giulia Marvaso ${ }^{1}$, Sara Gandini $^{2}$, Maddalena Mambretti ${ }^{1}$, Cristiana Fodor ${ }^{1}$, Dario Zerini ${ }^{1}$, Giulia Riva ${ }^{1}$, Damaris Patricia Rojas ${ }^{1}$, Giuseppe Petralia ${ }^{3}$, Raffaella Cambria ${ }^{4}$, Roberto Orecchia ${ }^{5}$ and Barbara Alicjia Jereczek ${ }^{1}$

${ }^{1}$ Department of Radiation Oncology, European

Institute of Oncology-IRCSS, Milan, Italy;

${ }^{2}$ Division of Epidemiology and Biostatistics,

European Institute of Oncology-IRCSS, Milan, Italy;
${ }^{3}$ Department of Radiology, European Institute of Oncology-IRCSS, Milan, Italy;

${ }^{4}$ Department of Medical Physics, European Institute of Oncology-IRCSS, Milan, Italy;

${ }^{5}$ Scientific Directorate, European Institute of Oncology-IRCSS, Milan, Italy

Aim: To evaluate the genitourinary (GU) and gastrointestinal (GI) toxicity after ultra-hypofractionated prostate cancer (PCa) radiotherapy. Materials and Methods: As a part of the prospective phase II Give-me-five trial, $65 \mathrm{PCa}$ patients underwent volumetric modulated arc therapy with simultaneous integrated boost to the dominant intraprostatic lesion (DIL). The treatment scheme was 36.25-37.5 Gy/5 fractions to the whole prostate gland and to the DIL, respectively. Univariate and multivariate logistic regression models were applied to identify independent factors associated with toxicity adjusting for confounding factors. The systematic analysis of urinary bladder and rectum dosevolume histograms (DVHs) was performed with the Fisher's exact test. The correlation between the relative volume receiving a given absolute dose and acute toxicity was investigated, with cutoffs at grade $\mathrm{G}>0$ and $\mathrm{G}>1$. Results: Sixty-five patients fulfilled the inclusion criteria. At 1 month after the end of radiotherapy, 42 patients $(65 \%)$ had no GU toxicity, whereas $18(28 \%), 4(6 \%)$ and $1(1 \%)$ patients had G1, G2 and G3 GU toxicity. As concerning GI toxicity, 54 $(83 \%)$ patients had no toxicity, whereas $11(17 \%)$ reported G1 toxicity. IPSS changed significantly $(p<0.05)$ from baseline to 1 month and changes during time are significantly different by symptomatic score at first month (mild, moderate and severe, $p<0.0001$ ). At univariate analysis (Table I), no relationship was found between the prostate volume and acute GU $(p=0.97)$ or GI toxicity $(p=0.83)$, nor between the urinary bladder volume and any grade of acute $\mathrm{GU}$ toxicity ( $p=0.60$ ) whereas the correlation between the rectum volume and any grade of acute GI toxicity was at the boundary of statistical significance $(p=0.05)$. At multivariate analysis adjusting for age and the presence of severe concomitant disease, IPSS at 1 month is positively significantly associated with acute $\mathrm{GU} G \geq 1$ toxicity $(p=0.03)$, whereas rectum volume is only borderline significantly associated with GI G $\geq 1$ toxicity $(p=0.06)$. Prostate volume is not significantly associated with any toxicity $(p=0.78)$. For urinary bladder, the area of DVH between 17-24 Gy vs. volume 12-22\% was correlated with any acute GU toxicity and the region between 25-35 Gy vs. volume $2-6 \%$ was correlated with acute G>1 GU toxicity. As concerning the relation between rectum DVH and GI toxicity, the portion between 18-34 Gy was correlated with any acute GI toxicity (Figure 1). Conclusion: The acute toxicity profile after ultra-hypofractionated radiotherapy for $\mathrm{PCa}$ is satisfactory. 

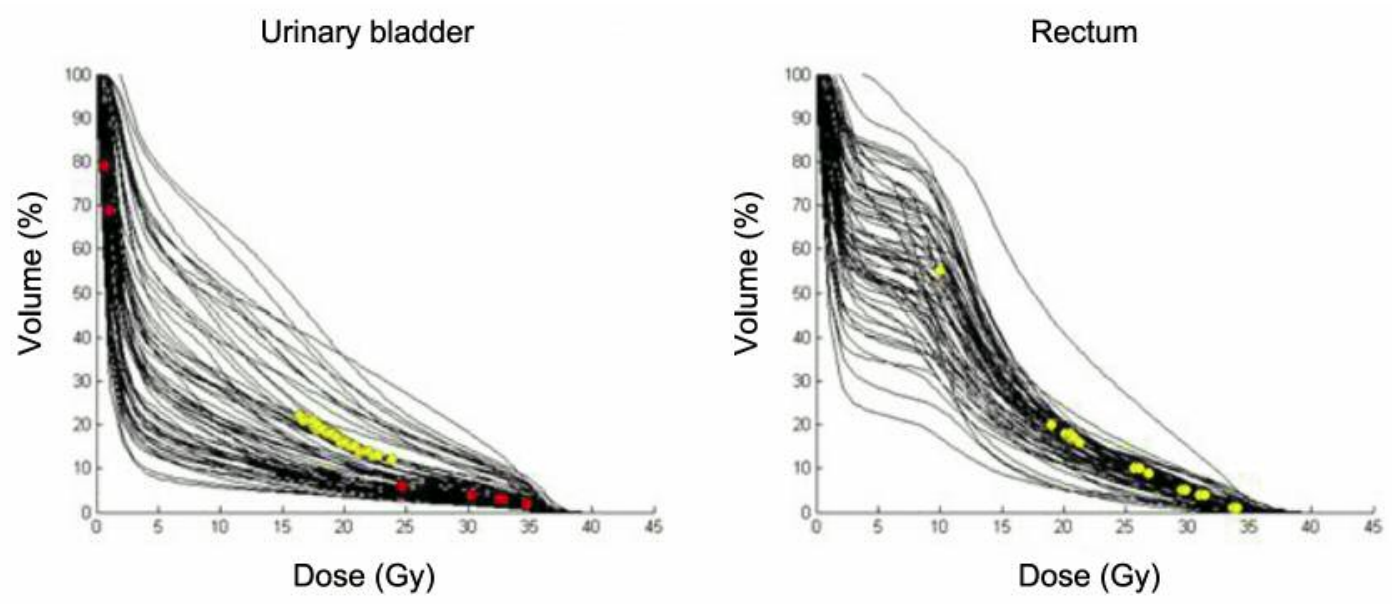

Figure 1. Dose-volume histograms of urinary bladder (left panel) and rectum (right panel) for all patients. Colored points represent dosevolume combinations that statistically significantly correlate with acute genito-urinary (left panel) and gastro-intestinal (right panel) toxicity at Fisher's Exact Test. In particular, yellow points refer to any-grade toxicity $(G>0)$ and red points refer to grade $G>1$ toxicity.

Table I. Univariate logistic regression evaluating the probability of acute genito-urinary (GU) and gastro-intestinal (GI) toxicity for urinary bladder and rectum, respectively. Values in the table represent median and interquartile range, in brackets.

\begin{tabular}{|c|c|c|c|c|c|}
\hline & & All & $\begin{array}{l}\text { No GU toxicity } \\
\qquad(\mathrm{n}=42)\end{array}$ & $\begin{array}{l}\text { Any GU toxicity } \\
\qquad(\mathrm{n}=23)\end{array}$ & $p$-Value \\
\hline \multirow[t]{2}{*}{ Urinary bladder } & $\begin{array}{l}\mathrm{V}_{18 \mathrm{~Gy}}(\%) \\
\mathrm{V}_{36 \mathrm{~Gy}}(\%) \\
\text { Bladder volume }\left(\mathrm{cm}^{3}\right) \\
\text { Prostate volume }\left(\mathrm{cm}^{3}\right)\end{array}$ & $\begin{array}{c}12.4(7.6,18) \\
0.8(0.36,1.3) \\
341(226,485) \\
43(33,56)\end{array}$ & $\begin{aligned} 12.1 & (7.6,20.9) \\
0.8 & (0.3,1.3) \\
354 & (211,470) \\
41 & (33,53)\end{aligned}$ & $\begin{array}{c}12.4(7.3,17.4) \\
0.7(0.36,1.3) \\
331(242,579) \\
44(33,58)\end{array}$ & $\begin{array}{l}0.366 \\
0.269 \\
0.602 \\
0.828\end{array}$ \\
\hline & & All & $\begin{array}{l}\text { No GI toxicity } \\
\quad(n=54)\end{array}$ & $\begin{array}{l}\text { Any GI toxicity } \\
\qquad(\mathrm{n}=11)\end{array}$ & $p$-Value \\
\hline Rectum & $\begin{array}{l}\mathrm{V}_{18 \mathrm{~Gy}}(\%) \\
\mathrm{V}_{29 \mathrm{~Gy}}(\%) \\
\mathrm{V}_{33 \mathrm{~Gy}}(\%) \\
\mathrm{V}_{36 \mathrm{~Gy}}(\%) \\
\text { Rectum volume }\left(\mathrm{cm}^{3}\right) \\
\text { Prostate volume }\left(\mathrm{cm}^{3}\right)\end{array}$ & $\begin{array}{c}20.9(16.4,23.5) \\
5.6(4.0,6.7) \\
1.5(0.85,2.9) \\
0.01(0.0,0.2) \\
62(50,82) \\
43(33,56)\end{array}$ & $\begin{array}{c}21(16.4,23.9) \\
5.8(4.5,7) \\
1.6(0.9,3) \\
0.02(0.0,0.3) \\
56(48,81) \\
43(35,53)\end{array}$ & $\begin{array}{c}19.6(14.9,21.2) \\
4.9(3.7,5.6) \\
1.1(0.6,1.6) \\
0.0(0.0,0.0) \\
77(69,94) \\
47(26,58)\end{array}$ & $\begin{array}{l}0.466 \\
0.164 \\
0.132 \\
0.146 \\
0.051 \\
0.969\end{array}$ \\
\hline
\end{tabular}

Abbreviations: $\mathrm{V}_{18}=$ Volume receiving $18 \mathrm{~Gy} ; \mathrm{V}_{29}=$ volume receiving $29 \mathrm{~Gy}$; V33=volume receiving $33 \mathrm{~Gy}$; $\mathrm{V}^{36}=$ volume receiving $36 \mathrm{~Gy}$; $\mathrm{GI}=$ genitourinary toxicity; GU=gastrointestinal toxicity.

37

EXTREME VERSUS MODERATE HYPOFRACTIONATION FOR LOCALIZED PROSTATE CANCER: A PROPENSITY SCORE-MATCHED ANALYSIS

Giulia Corrao $^{1}$, Giulia Marvaso ${ }^{1}$, Delia Ciardo $^{1}$, Sara Gandini ${ }^{2}$, Giulia Riva ${ }^{1}$, Emanuele Frigo $^{3}$, Cristiana Fodor ${ }^{1}$, Dario Zerini ${ }^{1}$, Paola Romanelli ${ }^{1}$, Stefania Comi ${ }^{4}$, Raffaella Cambria ${ }^{4}$, Ottavio De Cobelli ${ }^{5}$, Roberto Orecchia ${ }^{6}$ and Barbara Alicja Jereczek-Fossa ${ }^{1}$
${ }^{1}$ Division of Radiation Oncology, European Institute of Oncology-IRCCS, Milan, Italy; ${ }^{2}$ Division of Epidemiology and Biostatistics, European Institute of Oncology-IRCCS, Milan, Italy; ${ }^{3}$ Department of Oncology and Hemato-oncology, University of Milan, Milan, Italy; ${ }^{4}$ Unit of Medical Physics, European Institute of Oncology-IRCCS, Milan, Italy;

${ }^{5}$ Department of Urology, European Institute of Oncology-IRCCS, Milan, Italy;

${ }^{6}$ Scientific Directorate, European Institute of Oncology-IRCCS, Milan, Italy 
Aim: The aim of this study was to compare the clinical outcome and toxicity of two cohorts of clinically localized prostate cancer $(\mathrm{PCa})$ patients treated with two different radiotherapy $(\mathrm{RT})$ regimens: Extreme hypofractionation $(\mathrm{EH}$, 35 Gy or 32.5 Gy in 5 fractions) $v s$. moderate hypofractionation $(\mathrm{MH}$, Fox-Chase regimen, 70 Gy in 26 fractions), both using image-guided intensity modulated RT (IG-IMRT). Materials and Methods: Radiation Therapy Oncology Group/European Organization for Research and Treatment of Cancer criteria (RTOG/EORTC) and Houston definition (nadir+2) were used for toxicity and biochemical failure evaluation, respectively. Multivariate proportional Hazard Cox models, stratified for propensity score strata, were used to compare the 2 populations in terms of overall survival (OS), clinical progression-free survival (cPFS), biochemical PFS (bPFS) and gastro-intestinal (GI) toxicity and genitourinary $(\mathrm{GU})$ toxicity. Propensity scores reflect the probability that a patient received $\mathrm{MH}$ or $\mathrm{EH}$ based on his baseline characteristics. Patients receiving $\mathrm{MH}$ were matched on a oneto-one basis with subjects receiving EH. Matching was performed based on nearest-neighbor matching. The analyses were applied on the matched cohorts and on the whole cohorts, stratifying the analyses by risk strata identified with the propensity scores. Results: The analysis included $227 \mathrm{PCa}$ patients treated in 2007-2015, receiving MH-IG-IMRT and 194 patients treated with EH-IG-IMRT in 2012-2015. Median age was 75 years (range $=50-89$ ). Median follow-up was 43 months. A statistically significant difference in distribution regarding Gleason score and risk group was found between the two groups ( $p=0.004$ and $p=0.002$, respectively). Two-hundredtwenty-six patients with good matching characteristics and balance (113 men per group) were selected after propensity score matching. A multivariate Cox analysis performed on the pre-matched cohort showed that bPFS, cPFS and OS were not statistically different between the 2 treatment groups ( $p=0.439$, $p=0.317, p=0.906$, respectively), after adjusting for prognostic variables and confounding factors. These results were confirmed in the matched population analysis. As concerning GI toxicity, compared with $\mathrm{EH}$, in $\mathrm{MH}$ acute $\mathrm{G}>1$ events were significantly more frequent ( $3 \% v s .9 \%, p=0.007)$. Concerning GU toxicity, compared to $\mathrm{EH}$, in $\mathrm{MH}$, acute $\mathrm{G}>1$ events were registered in $11 \%$ vs. $26 \% \quad(p<0.001)$. Multivariate Cox regression models showed no significant differences between the cohorts in terms of late GI and GU toxicity. Acute GU/GI toxicities in the matched cohort were confirmed to be worse in the MH $(p=0.002$ and $p<0.001)$. The GU/GI toxicities detected at last follow-up were both in line with those emerged from the pre-matched analyses ( $p=0.43$ and $p=0.08$, respectively). Conclusion: Utilizing the propensity score comparison there was no difference in terms of oncological outcome at a median follow-up of 43 months comparing the two RT modalities. Moreover, the two RT regimens were associated with similar toxicity profiles.
38

XPERT BLADDER CANCER MONITOR IN THE FOLLOW-UP OF PATIENTS AFFECTED BY NON-MUSCLE INVASIVE BLADDER CANCER (NMIBC): AN UPDATE

Carolina D' ${ }^{\text {elia }}{ }^{1}$, Emanuela Trenti ${ }^{1}$, Alexander Pycha ${ }^{2}$, Christine Mian $^{3}$, Christine Schwienbacher ${ }^{3}$, Esther Hanspeter ${ }^{3}$ and Armin Pycha ${ }^{1}$

${ }^{1}$ Department of Urology, Central

Hospital of Bolzano, Bolzano, Italy;

${ }^{2}$ Department of Urology, Luzerner

Kantonsspital, Luzern, Switzerland;

${ }^{3}$ Department of Pathology, Central

Hospital of Bolzano, Bolzano, Italy

Introduction: Bladder cancer (BC) has one of the highest recurrence rates of any other tumour, ranging from 50-70\% within 5 years of the first treatment, requiring a lifelong follow-up with cystoscopy and cytology, limited for its low sensitivity in low-grade tumours. The Xpert Bladder Cancer Monitor kit is a new urinary marker test based on the evaluation of 5 target mRNAs (ABL1, CRH, IGF2, UPK1B and ANXA10), overexpressed in patient with BC. The aim of our study was to further evaluate the diagnostic accuracy of the Xpert BC Monitor test in the follow-up of patients with history of NMIBC and compare it with urinary cytology, cystoscopy and/or histology. Materials and Methods: A total of 290 patients under follow-up for NMIBC were included in this prospective study. Samples were analyzed with the Xpert BC Monitor kit and urinary cytology. Subsequently to urine collection, the patient underwent cystoscopy and if positive a TUR-B. Cytologies were evaluated according to the Paris System of reporting cytology. For statistical analysis, negative for high-grade urothelial cancer and atypical urothelial cells were grouped as negative, suspicious for high grade urothelial cancer, high grade urothelial cancer and low-grade urothelial neoplasia as positive. The Xpert BC Monitor test was reported by the software as negative or positive (cut-off total LDA=0.5). Sensitivity, specificity, PPV and NPV of Xpert BC Monitor and cytology were calculated using cystoscopy or histology results, if available, as a gold standard. Results: Median age of the patients was 73.5 years (range $=28-95$ years). Patients were followed-up for low-grade (LG) NMIBC in 188 cases and for high grade (HG) in 102 cases. One patient had to be excluded due to not diagnostic cytology and Xpert BC Monitor. Of the remaining 289 patients, 77 had tumour recurrence (60 LG (77.9\%), $17 \mathrm{HG}$ (22.1\%)). Overall sensitivity was $19.4 \%$ (15/77) for cytology, $48.05 \%(37 / 77)$ for Xpert ${ }^{\circledR}$ BC Monitor and 51.5\% (40/77) for the two tests combined. The sensitivity of cytology increased from $5 \%$ (3/60) in LG to $70.6 \%$ (13/17) in HG tumours whereas, for 
the Xpert ${ }^{\circledR}$ BC Monitor, the sensitivity was $40 \%$ (17/60) in LG and 76.5\% (13/17) in HG tumours. Combined cytology and Xpert ${ }^{\circledR}$ BC Monitor yielded an overall sensitivity of $41.6 \%$ (18/60) for LG and 94.1\% (16/17) for HG tumours. Overall specificity was $99.5 \%$ for cytology and $75.5 \%$ for Xpert $^{\circledR}$ BC. PPV for cytology was $93.7 \%$ and for Xpert ${ }^{\circledR}$ BC Monitor $42 \%$ while NPV was similar for the 2 tests: $77.3 \%$ for cytology versus $80 \%$ for Xpert ${ }^{\circledR}$ BC Monitor. Conclusion: The sensitivity of the Xpert BC Monitor Test is significantly higher than cytology, as previously reported. The test performs very well in terms of specificity, but cannot reach the extremely high value of cytology, PPV is significantly lower than for cytology, while NPV performs approximately the same for both tests.

\section{9}

\section{THE MODIFIED FRAILTY INDEX (MFI) VALUE IN UROLOGIC INTERVENTIONAL PROCEDURES: COMPARISON WITH ASA SCORE}

Vincenzo Serretta $^{1}$, Francesco Muffoletto ${ }^{1}$,

Gabriele Tulone $^{1}$, Salvatore Dioguardi ${ }^{1}$,

Calogero Guzzardo ${ }^{1}$, Sandro Billeci ${ }^{1}$, Sofia Giannone ${ }^{1}$,

Davide Baiamonte ${ }^{1}$, Cristina Scalici Gesolfo ${ }^{2}$, Chiara

Sanfilippo $^{3}$, Alchiede Simonato ${ }^{1}$ and

Members of the GSTU Foundation ${ }^{4}$

${ }^{1}$ Section of Urology, Department of Surgical, Oncological and Oral Sciences, University of Palermo, Palermo, Italy;

${ }^{2}$ Department of Urology, Paolo Borsellino

Hospital, Marsala, Italy;

${ }^{3}$ Statistics, GSTU Foundation, Palermo, Italy;

${ }^{4}$ Other Members, GSTU Foundation, Palermo, Italy

Introduction: Elderly patients are at increased risk for treatment-related toxicity secondary to medical comorbidities and geriatric syndromes. Almost $25 \%$ of the urological population is older than 75 years. Moreover, the frailty of a single individual might be not age-related. Development of methods and interventions of increased efficacy to reduce the morbidity from surgery are eagerly awaited. The ASA physical status classification system is a system for assessing the fitness of patients before surgery proposed by the American Society of Anesthesiologists (ASA) worldwide adopted. A frailty index predicting adverse outcomes among selected patients undergoing urologic oncological major surgeries was retrospectively validated by Lascano et al. (1) and subsequently simplified by Chappidi (2) as a preoperative predictor of complications following radical cystectomy. The aim of our prospective study was to test the modified frailty index (mFI) to identify those patients at greatest risk for complications among consecutive patients undergoing urological procedures for oncological and non-
Table I. Patients' characteristics.

\begin{tabular}{lc}
\hline & Patients \\
\hline Number & 247 \\
Gender & \\
Female & $44(18 \%)$ \\
Male & $203(82 \%)$ \\
Age & $68(57.25-73)$ \\
Codification of Operation & \\
1 & $51(21 \%)$ \\
2 & $129(52 \%)$ \\
3 & $43(17 \%)$ \\
4 & $23(10 \%)$ \\
Frailty Index & \\
0 & $61(25 \%)$ \\
1 & $86(35 \%)$ \\
2 & $60(24 \%)$ \\
3 & $32(13 \%)$ \\
4 & $5(2 \%)$ \\
5 & $3(1 \%)$ \\
ASA & \\
I & $5(2 \%)$ \\
II & $77(31 \%)$ \\
III & $162(66 \%)$ \\
IV & $3(1 \%)$ \\
BMI & $26.72(24.22-29.75)$ \\
Slbumin (g/dl) & $4.21(3.95-4.49)$ \\
Ex-smoker & \\
Smoker & $80(33 \%)$ \\
Non-smoker & $74(31 \%)$ \\
Serum Creatinine (mg/dl) & $85(36 \%)$ \\
\hline & $0.91(0.79-1.07)$ \\
\hline & \\
& \\
& \\
&
\end{tabular}

oncological diseases. Moreover, $\mathrm{mFI}$ was compared to ASA score. Materials and Methods: Consecutive patients undergoing urological procedures were prospectively entered. The surgical interventions were classified as follows: 1 . Major open/laparoscopic; 2 . Lower urinary tract endoscopy; 3. Upper urinary tract procedures; 4. Minor surgery. For all patients, age, ASA score, BMI, serum albumin, smoking history and routine hematological exams were preoperatively recorded. $\mathrm{mFI}$ was calculated. Operative time, hospital length of stay and post-operative complications according to Clavien-Dindo classification were recorded. Results: 247 consecutive patients, 203 men and 44 women underwent urological surgery. Age was over 75 years in 53 (21\%) patients. Patients' characteristics are given in Table I. While 239 (97\%) were assigned in ASA 2 and 3 categories, they resulted more widely distributed among the 5 MFI levels. Particularly of the 165 patients classified as ASA 3-4, 37 (22.4\%) only were allocated in 3$5 \mathrm{mFI}$ index and on the contrary of the 82 patients in ASA 1-2 classes, 79 (96.3\%) were allocated in 0-2 $\mathrm{mFI}$ categories. At univariate analysis both ASA and $\mathrm{mFI}$ were not associated 
with any complications $(p=0.76$ and $p=0.67)$, serious complications $(p=0.06$ and $p=0.49)$, and late complications rates $(p=0.46$ and $p=0.28)$. $\mathrm{mFI}$ was associated with age $(p<0.05)$ only, while ASA index only with age $(p<0.05)$, readmission rate $(p=0.03)$ and length of hospital stay $(p=0.004)$. Discussion: A correspondence between ASA and $\mathrm{mFI}$ emerges only for low risk classes, since $22 \%$ only of the patients classified as ASA 3-4 resulted allocated in the corresponding high-risk classes of mFI. Both $\mathrm{mFI}$ and ASA were not associated with complication incidence when oncological and non-oncological urologic surgery is considered.

The Authors thank GSTU Foundation, Palermo, Italy for the editing and statistical support.

1 Lascano D, Pak JS, Kates M, Finkelstein JB, Silva M, Hagen E, RoyChoudhury A, Bivalacqua TJ, DeCastro GJ, Benson MC and McKiernan JM: Validation of a frailty index in patients undergoing curative surgery for urologic malignancy and comparison with other risk stratification tools. Urol Oncol 33: 426.e1-12, 2015. PMID: 26163940. DOI: 10.1016/j.urolonc.2015.06.002

2 Chappidi MR, Kates M, Patel HD, Tosoian JJ, Kaye DR, Sopko NA, Lascano D, Liu JJ, McKiernan J and Bivalacqua TJ: Frailty as a marker of adverse outcomes in patients with bladder cancer undergoing radical cystectomy. Urol Oncol 34: 256e1-6, 2016. PMID: 26899289. DOI: $10.1016 /$ j.urolonc.2015.12.010

40

REAL-TIME TRANS-RECTAL ULTRASOUNDGUIDED ROBOT-ASSISTED TRANS-PERINEAL RADICAL PROSTATECTOMY: A PRE-CLINICAL EXPERIENCE USING THE SP ${ }^{\circledR}$ SURGICAL SYSTEM

Riccardo Bertolo, Juan Garisto,

Mohamed Eltemamy and Jihad Kaouk

Glickman Urological and Kidney Institute,

Cleveland Clinic, Cleveland, OH, U.S.A.

Introduction: The FDA approved da Vinci $\mathrm{SP}^{\circledR}$ (Intuitive Surgical, Sunnyvale, CA) surgical system was developed specifically to facilitate single-site surgery. The compact profile of the platform's working element is proper to operate within smaller working spaces so that the feasibility of "unconventional" approaches to various urologic interventions has been investigated in a pre-clinical setting (1). Noteworthy, alternative access routes for prostatectomy have been rediscovered, including the trans-perineal approach. Open surgery literature reported that the recovery of postoperative urinary continence is mostly immediate with this approach, due to preserved urethra-sphincter

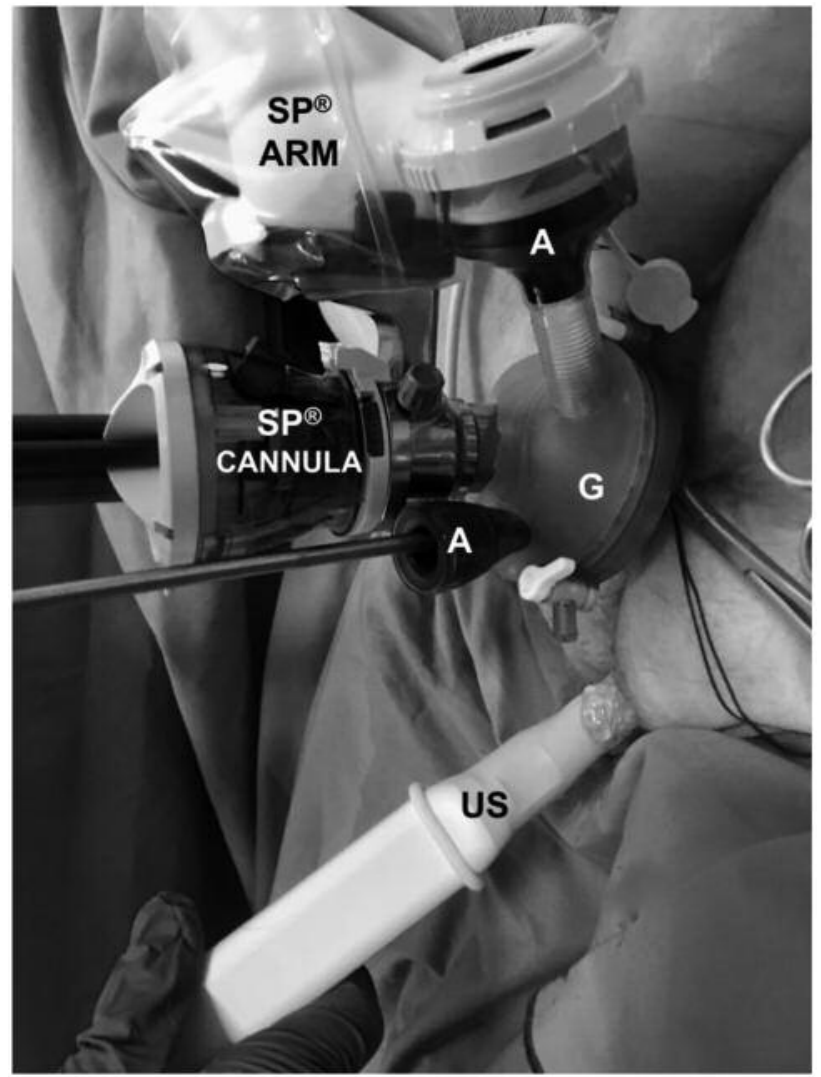

Figure 1. Final setup during real-time trans-rectal ultrasoundguided robot-assisted trans-perineal radical prostatectomy. $A=$ Assistant port; G=GelPOINT membrane; US=trans-rectal ultrasound probe.

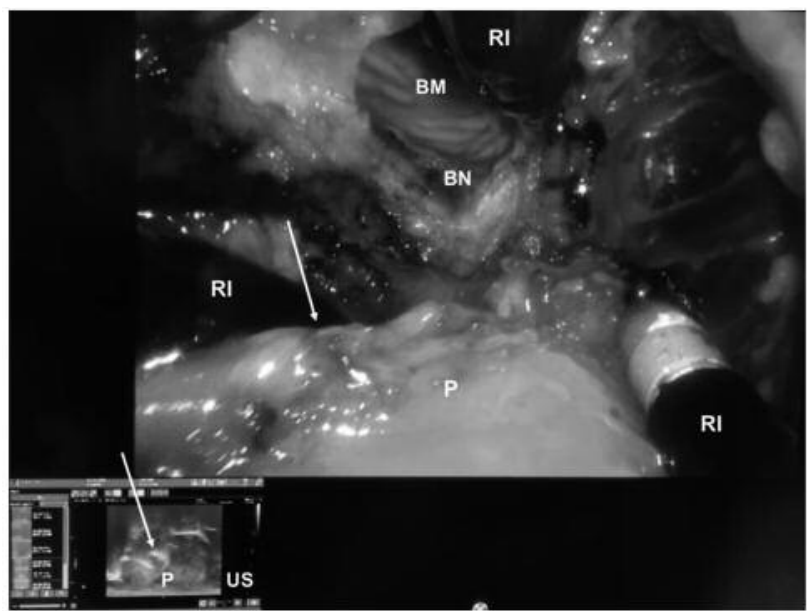

Figure 2. Picture in picture during real-time trans-rectal ultrasound-guided robot-assisted trans-perineal radical prostatectomy (surgeon's view). The arrows underline the robotic instrument pushing down the prostate that is hyperechoic at the ultrasonography. US=Ultrasound; P=Prostate; $R I=$ robotic instrument; $B N=$ bladder neck; BM=bladder mucosa. 
complex anatomy (2). On the other hand, trans-perineal prostatectomy represents a challenging surgery: the achievement of the right plane for an anatomical dissection can be tricky. We aimed to report a pre-clinical experience with real-time trans-rectal ultrasound (TRUS)-guided pure single-site trans-perineal robotic radical prostatectomy performed by the da Vinci $\mathrm{SP}^{\circledR}$ surgical system. Materials and Methods: One fresh male cadaver was used as a model for trans-perineal radical prostatectomy performed by the da Vinci $\mathrm{SP}^{\circledR}$ surgical system. The surgery was completed via a $2.5 \mathrm{~cm}$ incision performed between the ischial tuberosities through which a GelPOINT advanced access platform (Applied Medical, Rancho Santa Margarita, CA, USA) and a dedicated 25-mm multi-channel port accommodating a $12 \times 10-\mathrm{mm}$ articulating robotic camera, three $6-\mathrm{mm}$ double-jointed articulating robotic instruments and a 6-mm accessory laparoscopic instrument were placed. Assistant ports were placed through the GelPOINT membrane. The Trinity ${ }^{\circledR}$ (Koelis, Meylan, France) ultrasound system for real-time 3D TRUS was used to guide the prostatectomy (Figure 1). The steps in the procedure included the division of the rectourethralis muscle, the splitting of the levator and muscles bilaterally, the opening of Denonvilliers fascia with dissection of the seminal vesicles, the apical dissection and the division of the urethra, the anterior and lateral dissection with ligation of the prostatic pedicles, and the performance of the vesicourethral anastomosis (2). The goal of the study was to test the feasibility of the TRUS-guided approach and to evaluate the eventual benefits of the image-guidance during the surgery. Results: Pure single-site trans-perineal robotic radical prostatectomy was successfully performed by using the da Vinci $\mathrm{SP}^{\circledR}$ surgical system. Real-time TRUSguidance helped in delineating the prostate boundaries (Figure 2). Particularly, with respect to the non-TRUSguided procedure, the prostate apex was promptly identified. Discussion and Conclusion: The feasibility of real-time TRUS-guided pure single-site trans-perineal robotic radical prostatectomy performed by the da Vinci $\mathrm{SP}^{\circledR}$ surgical system is reported herein. The use of TRUSguidance could reduce the challenges related with the approach and open the doors to such indication. Further studies are needed to confirm our findings. Reproducibility in the clinical model is warranted.

1 Bertolo R, Garisto J, Gettman M and Kaouk J. Novel system for robotic single-port surgery: feasibility and state of the art in urology. Eur Urol Focus 4: 669-673, 2018. PMID: 29914841. DOI: 10.1016/j.euf.2018.06.004

2 Ramirez D, Maurice MJ and Kaouk JH. Robotic perineal radical prostatectomy and pelvic lymph node dissection using a purpose-built single-port robotic platform. BJU Int 118: 829-833, 2016. PMID: 27410172. DOI: 10.1111/bju.13581
41

\section{ACTIVE SURVEILLANCE FOR SMALL RENAL MASSES 2CM: RESULTS FROM AN ITALIAN MULTI-INSTITUTIONAL PROSPECTIVE PROTOCOL}

Walter Cazzaniga $^{1}$, Fabio Castiglione ${ }^{1}$, Alessandro Larcher ${ }^{1}$, Alessandro Nini ${ }^{1}$, Cristina Carenzi ${ }^{1}$, Rayan Matloob ${ }^{1}$, Luca Villa $^{1}$, Gianfranco Conti ${ }^{2}$, Carmen Maccagnano ${ }^{2}$, Claudio Simeone ${ }^{3}$, Alessandro Antonelli ${ }^{3}$, Emanuele Montanari ${ }^{4}$, Giancarlo Albo ${ }^{4}$, Roberto Salvioni ${ }^{5}$, Silvia Stagni ${ }^{5}$, Mario Catanzaro ${ }^{5}$, Umberto Capitanio ${ }^{1}$, Francesco Montorsi ${ }^{1}$ and Roberto Bertini ${ }^{1}$

${ }^{1}$ Division of Urology, Division of Experimental Oncology/Unit of Urology, URI-Urological Research Institute, IRCCS Ospedale San Raffaele, Milan, Italy; ${ }^{2}$ Department of Urology, Azienda Ospedaliera Sant' Anna di Como, Como, Italy; ${ }^{3}$ Department of Medical and Surgical Specialties, Radiological Sciences, and Public Health, Spedali Civili Hospital, University of Brescia, Brescia, Italy; ${ }^{4}$ Department of Urology, IRCCS Fondazione Ca'GrandaOspedale Maggiore Policlinico University of Milan, Milan, Italy;

${ }^{5}$ Department of Urology, Fondazione IRCCS

Istituto Nazionale dei Tumori, Milan, Italy

Introduction: Small renal masses (SRMs) have become increasingly common due to the extensive adoption of imaging exams. Numerous treatment approaches such as surgical interventions, tissue ablation and active surveillance have all been proved to play a role in the management of SRMs. In this study, we report the first descriptive results from this prospective, non-randomized, multi- institutional protocol for the active surveillance of SRMs $\leq 2 \mathrm{~cm}$. Patients and Methods: The protocol has been introduced in April 2014 in 5 Italian centers. Patients with monolateral SRM $(2 \mathrm{~cm}$ of diameters), with more than 50 years of age and without symptoms related to the mass have been enrolled in the protocol. Patients with previous history of renal cancer, single kidney, hereditary tumors, presence of metastasis, immunodepressant therapy or with a life expectancy lower than 1 year have been considered not eligible. All the patients underwent a standardized follow up scheme and collection of urine and blood samples for the creation of a biobank of biological samples. Results: To date, 59 patients have been enrolled in the protocol, 2/59 (3.4\%) have dropped out from the study and $0 / 59$ have died in the study period. The mean follow-up time was 24.7 months $(\mathrm{SD}=8.6)$. No patients have developed symptoms or paraneoplastic syndromes during the follow up period. At the enrolment, the mean linear diameter was $1.3 \mathrm{~cm}(\mathrm{SD}=0.36)$ and the mean volume was $1.8 \mathrm{~cm}^{3}$ $(\mathrm{SD}=1.22)$. The mean annual increase in the linear growth has 
been $0.13 \mathrm{~cm} /$ year and the mean annual increase in the volumetric growth has been $0.71 \mathrm{~cm}^{3} /$ year. $3 / 59(5 \%)$ have been submitted to percutaneous biopsy and the cause was the tumor growth (2 RCC treated with thermal ablation and 1 angiomyolipoma, not treated). Furthermore, 2/59 (3.4\%) patients are currently listed for a partial nephrectomy. Overall, $4 / 59(6.8 \%)$ patients have required active treatment due to tumour growth. Conclusion: Our preliminary data suggests the feasibility and the safety of the introduction of an active surveillance protocol for small renal masses. Also, in our experience, this approach may represent a valuable option to offer to patients older than 50 years old and diagnosed with an SRM $2 \mathrm{~cm}$. The biomolecular aspects of these masses will be evaluated in future studies.

\section{3}

\section{METASTASES-DIRECTED STEREOTACTIC RADIOTHERAPY FOR OLIGOMETASTATIC CASTRATION-RESISTANT PROSTATE CANCER: MULTICENTRE SERIES OF 86 PATIENTS/117 LESIONS}

Marco Lorenzo Bonù ${ }^{1}$, Luca Triggiani ${ }^{1}$, Davide Tomasini ${ }^{1}$, Gianluca Ingrosso ${ }^{2}$, Paolo Borghetti ${ }^{3}$, Fabio Trippa ${ }^{4}$, Andrea Lancia ${ }^{2}$, Beatrice Detti ${ }^{5}$, Fabio Matrone ${ }^{6}$, Giuseppe Fanetti ${ }^{7}$, Francesco Pasqualetti ${ }^{8}$, Alessandro Magli ${ }^{9}$, Alessio Bruni ${ }^{10}$, Ercole Mazzeo ${ }^{10}$, Ciro Franzese ${ }^{11}$, Guido Francolini ${ }^{5}$ and Rosario Mazzola ${ }^{12}$

${ }^{1}$ Radiation Oncology Department, University and Spedali Civili Hospital, Brescia, Italy; ${ }^{2}$ Department of Radiation Oncology, Policlinico Tor Vergata, University of Rome, Rome, Italy; ${ }^{3}$ Radiation Oncology Unit, University and Spedali Civili Hospital, Brescia, Italy;

${ }^{4}$ Radiation Oncology, Radiation Oncology

Centre, "S. Maria" Hospital, Terni, Italy;

${ }^{5}$ Department of Radiation Oncology,

University of Florence, Azienda

Ospedaliero-Universitaria, Careggi, Italy;

${ }^{6}$ Radiotherapy Unit, CRO IRCCS, Aviano, Italy;

7Division of Radiotherapy, European

Institute of Oncology, Milan, Italy;

${ }^{8}$ Department of Radiation Oncology, University

Hospital S. Chiara, Pisa, Italy;

${ }^{9}$ Department of Radiation Oncology, University

Hospital of Udine, ASUIUD, Udine, Italy;

${ }^{10}$ Department of Radiation Oncology, AOU Policlinico

Modena, Modena, Italy;

${ }^{11}$ Radiotherapy and Radiosurgery Department,

Humanitas Research Hospital, Milan-Rozzano, Italy;

${ }^{12}$ Radiation Oncology Department, IRCCS, Sacro Cuore

Don Calabria Hospital, Negrar-Verona, Italy
Introduction: Herein, we report the clinical outcomes of a multicentre study evaluating the role of SBRT in a cohort of patients affected by oligometastatic castration-resistant prostate cancer (CRPC). Materials and Methods: This is a retrospective multicenter observational study including eleven centers. Inclusion criteria of the current study were: a) Karnofsky performance status $>80$, b) histologically proven diagnosis of PC, c) 1-5 oligoprogressive metastases, defined as progressive disease at bone or nodes levels (detected by means of choline PET/TC or CT plus bone scan) during ADT, d) serum testosterone level under $50 \mathrm{ng} / \mathrm{ml}$ during ADT, e) controlled primary tumor, f) patients treated with SBRT with a dose of at least $5 \mathrm{~Gy}$ per fraction to a biologically effective dose (BED) of at least $80 \mathrm{~Gy}$ using an alpha/beta ratio of $3 \mathrm{~Gy}$, g) at least 6 months of follow up post-SBRT. Results: 86 patients for a total of 117 lesions were treated with SBRT. The median follow-up was 30.7 months (range=4-91 months). The median metastases-free survival after SBRT was 12.3 months (95\% CI=5.5-19.1 months). One- and two-years distant progression free survival was $52.3 \%$ and $33.7 \%$ respectively. Twenty-six out of 86 patients underwent a second course of SBRT due to further oligoprogressive disease: this resulted in a median systemic treatment-free survival of 21.8 months (95\% CI: 17.8-25.8 months). One-year systemic treatment-free survival was $72.1 \%$. Conclusion: SBRT represents an important tool able to impact in the natural history of oligometastatic castration-resistant prostate cancer.

\section{4 \\ WHICH PATIENT WITH BIOCHEMICAL RECURRENCE AFTER PRIMARY TREATMENT FOR PROSTATE CANCER WOULD RESULT IN A POSITIVE ${ }^{68}$ GA-PSMA PET/CT? A CLINICAL TOOL TO GUIDE PHYSICIANS BEFORE SUGGESTING ${ }^{68}$ GA-PSMA PET/CT}

\author{
Lorenzo Bianchi ${ }^{1}$, Francesco Ceci ${ }^{2}$, Marco Borghesi ${ }^{1}$, \\ Giulia Polverari ${ }^{3}$, Federico Mineo Bianchi ${ }^{1}$, \\ Francesco Chessa ${ }^{1}$, Paolo Castellucci ${ }^{3}$, \\ Stefano Fanti ${ }^{2}$ and Eugenio Brunocilla ${ }^{1}$ \\ ${ }^{1}$ Division of Urology, Sant'Orsola Malpighi Hospital - \\ University of Bologna, Bologna, Italy; \\ ${ }^{2}$ Division of Nuclear Medicine, Bologna, Italy; \\ ${ }^{3}$ Division of Nuclear Medicine, Sant'Orsola Malpighi \\ Hospital - University of Bologna, Bologna, Italy
}

Introduction and Objectives: To develop a clinical nomogram aimed to predict which patients with recurrent prostate cancer (PCa) could benefit from ${ }^{68}$ Gallium-Prostate Specific Membrane Antigen Positron Emission Tomography/ Computed Tomography $\left({ }^{68} \mathrm{Ga}-\mathrm{PSMA}\right.$ PET/CT). Materials and Methods: We retrospectively enrolled 703 PCa patients with 


\section{Overall population $(n=703)$}

Points

0

ISUP group

PSA at PSMA PET/CT

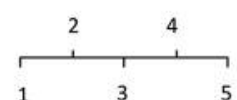

$\begin{array}{lll}1 & 3 & 5\end{array}$

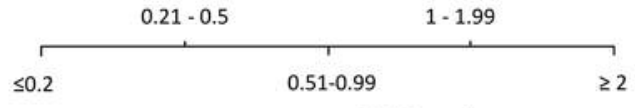

PSA doubling time

6-11.9 months $<3$ months

212 months $3-5.99$ months

On-going ADT

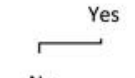

No

Time to PSA recurrence

Total points

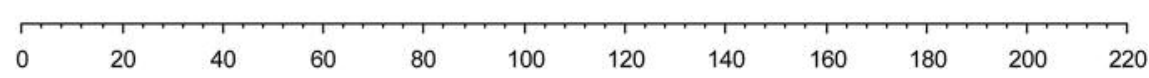

Likelihood of positive scan*

$\begin{array}{lllllllll}0.1 & 0.2 & 0.3 & 0.4 & 0.5 & 0.6 & 0.7 & 0.8 & 0.9\end{array}$

${ }^{*}$ AUC of the prediction model $=0.823$

Figure 1. Nomogram to predict the likelihood of positive ${ }^{68}$ Ga-PSMA PET/CT.

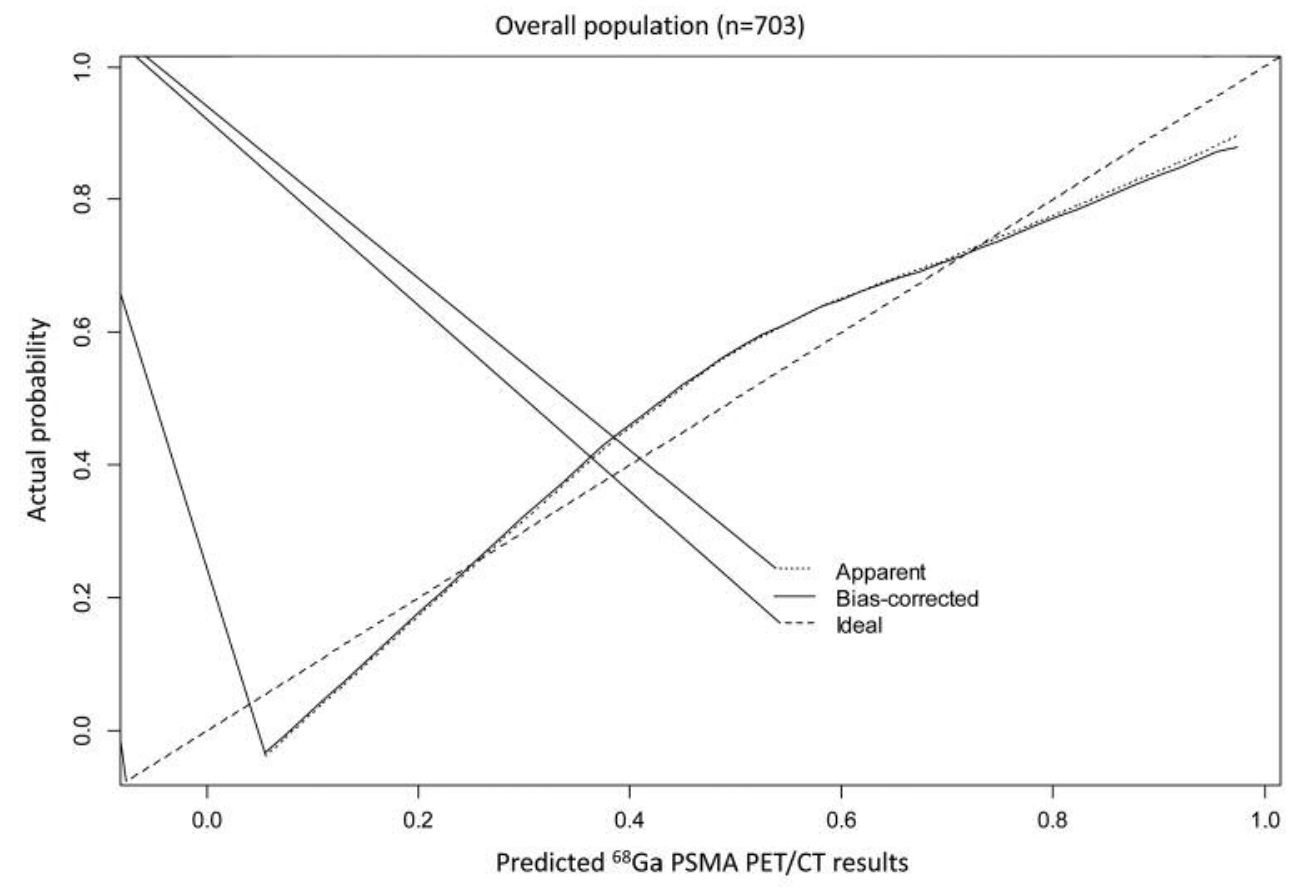

Figure 2. Calibration plot of the nomogram. 
Table I. Overall patient characteristics.

\begin{tabular}{|c|c|}
\hline Variable & Overall \\
\hline No. of patients & 703 \\
\hline \multicolumn{2}{|l|}{ Age (years) } \\
\hline Median & 68 \\
\hline IQR & $63-73$ \\
\hline \multicolumn{2}{|l|}{ Primary treatment, $\mathrm{n}(\%)$} \\
\hline Radical prostatectomy & $684(97.3)$ \\
\hline Radiotherapy & $19(2.7)$ \\
\hline \multicolumn{2}{|l|}{ ISUP $\operatorname{grade}^{\#}, \mathrm{n}(\%)$} \\
\hline 1 & $67(9.5)$ \\
\hline 2 & $128(18.2)$ \\
\hline 3 & $223(31.7)$ \\
\hline 4 & $155(22)$ \\
\hline 5 & $130(18.5)$ \\
\hline \multicolumn{2}{|l|}{$\mathrm{T}$ stage $\mathrm{e}^{\S}, \mathrm{n}(\%)$} \\
\hline Missing data & $70(10)$ \\
\hline $\mathrm{T} 2-\mathrm{T} 3 \mathrm{a}$ & $231(32.9)$ \\
\hline T3b-pT4 & $402(57.2)$ \\
\hline \multicolumn{2}{|l|}{ PLND, n $(\%)^{*}$} \\
\hline No & $148(21.6)$ \\
\hline Yes & $536(78.4)$ \\
\hline \multicolumn{2}{|l|}{ Nodal status, $\mathrm{n}(\%)^{*}$} \\
\hline Missing data & $62(9.1)$ \\
\hline pNO & $508(74.3)$ \\
\hline pN1 & $114(16.7)$ \\
\hline \multicolumn{2}{|c|}{ Positive surgical margins, $\mathrm{n}(\%)^{*}$} \\
\hline Missing data & $101(14.8)$ \\
\hline No & $342(50)$ \\
\hline Yes & $241(35.2)$ \\
\hline \multicolumn{2}{|c|}{ Adjuvant radiotherapy, $\mathrm{n}(\%)^{*}$} \\
\hline No & $502(73.4)$ \\
\hline Yes & $182(26.6)$ \\
\hline \multicolumn{2}{|c|}{ Salvage radiotherapy, $\mathrm{n}(\%)^{*}$} \\
\hline No & $515(75.2)$ \\
\hline Yes & $169(24.7)$ \\
\hline \multicolumn{2}{|c|}{ PSA at ${ }^{68} \mathrm{Ga}-\mathrm{PSMA}$ PET/CT $(\mathrm{ng} / \mathrm{ml})$} \\
\hline Median & 0.7 \\
\hline IQR & $0.4-1.3$ \\
\hline
\end{tabular}

confirmed biochemical recurrence (BCR) after radical prostatectomy $(n=684)$ and primary radiotherapy $(n=19)$. Each man underwent ${ }^{68} \mathrm{Ga}$-PSMA PET/CT to identify the site of recurrence. Patients were stratified according to different clinical settings of recurrence (First PSA relapse, BCR after salvage therapy, PSA persistence after primary therapy and disease progression before starting systemic therapies in 325 , 241, 76 and 61 patients, respectively). First, we assessed the detection rate of ${ }^{68} \mathrm{Ga}$-PSMA PET/CT in overall population and in each subgroup of patients. Second, multivariate logistic regressions were used to determine which co-variates (including ISUP group, PSA at ${ }^{68} \mathrm{Ga}$-PSMA PET/CT, PSA doubling time, ongoing androgen deprivation therapy (ADT) at ${ }^{68} \mathrm{Ga}$-PSMA PET/CT, time to recurrence and the clinical

\begin{tabular}{|c|c|}
\hline Variable & Overall \\
\hline \multicolumn{2}{|c|}{ PSA ranges at ${ }^{68} \mathrm{Ga}-\mathrm{PSMA}$ PET/CT $(\mathrm{ng} / \mathrm{ml}), \mathrm{n}(\%)$} \\
\hline$\leq 0.2$ & $30(4.3)$ \\
\hline $0.21-0.5$ & $213(30.3)$ \\
\hline $0.6-1$ & $203(28.9)$ \\
\hline $1.1-2$ & $167(23.8)$ \\
\hline$>2$ & $90(12.8)$ \\
\hline \multicolumn{2}{|l|}{ On-going ADT at ${ }^{68} \mathrm{Ga}$-PSMA PET/CT, n (\%) } \\
\hline No & $587(83.5)$ \\
\hline Yes & $116(16.5)$ \\
\hline \multicolumn{2}{|l|}{ Time to BCR (months) } \\
\hline Median & 21 \\
\hline IQR & $8.8-49$ \\
\hline \multicolumn{2}{|l|}{ Time to BCR (months) } \\
\hline$>12$ months & $492(70)$ \\
\hline$\leq 12$ months & $211(30)$ \\
\hline \multicolumn{2}{|l|}{ PSA doubling time (months) } \\
\hline Median & 6 \\
\hline IQR & $3.5-9.6$ \\
\hline \multicolumn{2}{|l|}{ PSA doubling time (months), $\mathrm{n}(\%)$} \\
\hline$<3$ months & $132(18.8)$ \\
\hline 3-6 months & $215(30.6)$ \\
\hline 6-12 months & $230(32.7)$ \\
\hline$>12$ months & $126(17.9)$ \\
\hline \multicolumn{2}{|l|}{${ }^{68} \mathrm{Ga}$-PSMA PET/CT results, $\mathrm{n}(\%)$} \\
\hline Positive & $360(51.2)$ \\
\hline Negative & $343(48.8)$ \\
\hline \multicolumn{2}{|l|}{ Clinical setting, n (\%) } \\
\hline First PSA recurrence & $325(46.2)$ \\
\hline PSA recurrence after salvage therapy & $241(46.2)$ \\
\hline PSA persistence after surgery & $76(10.8)$ \\
\hline Assessment before systemic treatments & $61(8.7)$ \\
\hline
\end{tabular}

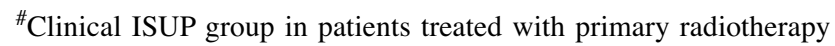
and pathologic ISUP group in patients treated with primary radical prostatectomy; ${ }^{\S}$ Clinical stage in patients treated with primary radiotherapy and pathologic stage in patients treated with primary radical prostatectomy; ${ }^{*}$ Considering patients referred to radical prostatectomy as primary treatment.

setting) independently predict a positive ${ }^{68} \mathrm{Ga}$-PSMA PET/CT result. Finally, regression-based coefficients were used to develop a nomogram (Figure 1) predicting positive ${ }^{68} \mathrm{Ga}-$ PSMA PET/CT result and 200 bootstrap resamples were used for internal validation. Results: Table I shows patients' perioperative characteristics. Median PSA at ${ }^{68} \mathrm{Ga}-\mathrm{PSMA}$ $\mathrm{PET} / \mathrm{CT}$ was $0.7 \mathrm{ng} / \mathrm{ml}$ (IQR=0.4-1.3). Overall, 116 (16.5\%) men were on-going with ADT at the time of ${ }^{68} \mathrm{Ga}-\mathrm{PSMA}$ $\mathrm{PET} / \mathrm{CT}$. Median time to BCR was 21 months (IQR=9-49); median PSA doubling time was 6 months (IQR=4-10). The overall detection rate of ${ }^{68} \mathrm{Ga}$-PSMA PET/CT was $51.2 \%$. Table II reports PET-CT diagnostic performance among the different subgroups: detection rate was 40.3\%, 54\%, $60.5 \%$ and $86.9 \%$ in patients with first PSA relapse, BCR after 
Table II. Patient characteristics and detection rates according to different clinical setting.

\begin{tabular}{|c|c|c|c|c|c|c|c|}
\hline $\begin{array}{l}\text { Different clinical } \\
\text { stages of BCR }\end{array}$ & $\begin{array}{l}\text { No. of } \\
\text { patients }\end{array}$ & $\begin{array}{l}\text { Mean PSA } \\
(\mathrm{ng} / \mathrm{mL})\end{array}$ & $\begin{array}{l}\text { Detection } \\
\text { rate }\end{array}$ & $\begin{array}{l}\text { Pelvic } \\
\text { relapse* }\end{array}$ & $\begin{array}{l}\text { Distant } \\
\text { relapse** }\end{array}$ & $\begin{array}{l}\text { Oligometastatic } \\
\text { (1 to } 3 \text { lesions) }\end{array}$ & $\begin{array}{c}\text { Multimetastatic } \\
\text { (>3 lesions) }\end{array}$ \\
\hline \multicolumn{8}{|l|}{ Group-1 } \\
\hline $\begin{array}{l}\text { First-time BCR } \\
\text { (no salvage therapy) } \\
\text { Group-2 }\end{array}$ & 325 & 0.9 & $40.3 \%$ & $19.4 \%$ & $20.9 \%$ & $35.7 \%$ & $4.6 \%$ \\
\hline $\begin{array}{l}\text { PSA failure after salvage therapy } \\
\text { Group-3 }\end{array}$ & 241 & 1.3 & $54.0 \%$ & 21.6 & $32.4 \%$ & $42.8 \%$ & $11.2 \%$ \\
\hline $\begin{array}{l}\text { Biochemical Persistence post-RP } \\
\text { Group-4*** }\end{array}$ & 76 & 1.1 & $60.5 \%$ & $31.6 \%$ & $28.9 \%$ & $39.5 \%$ & $21 \%$ \\
\hline Restaging before systemic therapy & 61 & 4.7 & $86.9 \%$ & $8.2 \%$ & $78.7 \%$ & $36 \%$ & $50.9 \%$ \\
\hline p-Value & - & $<0.001$ & $<0.001$ & 0.3 & $<0.001$ & 0.2 & $<0.001$ \\
\hline Overall Population & 703 & 1.3 & $51.2 \%$ & $20.4 \%$ & $30.8 \%$ & $38.5 \%$ & $12.7 \%$ \\
\hline
\end{tabular}

*Prostate bed and/or pelvic lymph-nodes. **At least one distant lesion (extra-pelvic lymph nodes and/or bone and/or visceral metastasis). ***Chemotherapy and/or abiraterone/enzalutamide and/or radioligand-therapy naïve.

Table III. Uni and multivariate logistic regression to predict positive findings at PSMA PET/CT ( $n=703)$.

\begin{tabular}{|c|c|c|c|c|}
\hline \multirow[t]{2}{*}{ Variables } & \multicolumn{2}{|c|}{ Univariate } & \multicolumn{2}{|c|}{ Multivariate } \\
\hline & OR $(95 \% \mathrm{CI})$ & $p$-Value & OR $(95 \% \mathrm{CI})$ & $p$-Value \\
\hline \multicolumn{5}{|l|}{ ISUP group } \\
\hline 1 & 1.0 (Ref) & & $1.0(\operatorname{Ref})$ & \\
\hline 2 & $1.15(0.63-2.11)$ & 0.7 & $1.34(0.64-2.82)$ & 0.4 \\
\hline 3 & $1.82(1.04-3.19)$ & 0.04 & $2.02(1.02-3.99)$ & 0.04 \\
\hline 4 & $2.15(1.19-3.87)$ & 0.01 & $1.76(0.86-3.59)$ & 0.01 \\
\hline 5 & $2.69(1.46-4.94)$ & 0.001 & $2.35(1.12-4.94)$ & 0.02 \\
\hline \multicolumn{5}{|l|}{ PSA at PSMA PET/CT (ng/ml) } \\
\hline$\leq 0.2$ & $1.0(\operatorname{Ref})$ & & $1.0(\operatorname{Ref})$ & \\
\hline $0.21-0.49$ & $1.79(0.73-4.36)$ & 0.2 & $1.83(0.67-4.97)$ & 0.2 \\
\hline $0.5-0.99$ & $4.21(1.73-10.25)$ & 0.002 & $4.52(1.66-12.26)$ & 0.003 \\
\hline $1-1.99$ & $5.57(2.26-13.72)$ & $<0.001$ & $6.09(2.20-16.87)$ & 0.001 \\
\hline$\geq 2$ & $6.25(2.42-16.19)$ & $<0.001$ & $5.39(1.78-16.35)$ & 0.003 \\
\hline \multicolumn{5}{|l|}{ PSA doubling time $(\mathrm{ng} / \mathrm{ml} /)$} \\
\hline$<3$ months & 1.0 (Ref) & & 1.0 (Ref) & \\
\hline 3-5.9 months & $0.56(0.33-0.95)$ & 0.03 & $0.59(0.34-1.04)$ & 0.07 \\
\hline 6-11.9 months & $0.15(0.09-0.25)$ & $<0.001$ & $0.16(0.09-0.28)$ & $<0.001$ \\
\hline$\geq 12$ months & $0.03(0.01-0.05)$ & $<0.001$ & $0.02(0.11-0.06)$ & $<0.001$ \\
\hline \multicolumn{5}{|l|}{ Ongoing ADT at BCR } \\
\hline No & $1.0($ Ref $)$ & & $1.0(\operatorname{Ref})$ & \\
\hline Yes & $2.68(1.74-4.13)$ & $<0.001$ & $1.04(0.56-1.93)$ & 0.9 \\
\hline \multicolumn{5}{|l|}{ Time to BCR (months) } \\
\hline$>12$ months & $1.0(\operatorname{Ref})$ & & $1.0(\operatorname{Ref})$ & \\
\hline$\leq 12$ months & $1.39(1.00-1.92)$ & 0.049 & $1.03(0.64-1.68)$ & 0.9 \\
\hline \multicolumn{5}{|l|}{ Clinical setting } \\
\hline First recurrence after primary treatment & $1.0(\operatorname{Ref})$ & & $1.0(\operatorname{Ref})$ & \\
\hline Recurrence after salvage therapy & $1.73(1.24-2.43)$ & 0.001 & $1.30(0.85-1.98)$ & 0.2 \\
\hline PSA persistence after primary treatment & $2.27(1.36-3.78)$ & 0.002 & $1.43(0.70-2.90)$ & 0.3 \\
\hline Advanced stage PCa & $9.81(4.51-21.31)$ & $<0.001$ & $6.05(2.14-17.11)$ & 0.001 \\
\hline Predictive accuracy & - & & $82.3 \%$ & \\
\hline
\end{tabular}

ADT: Androgen deprivation therapy; BCR: biochemical recurrence; PSA: prostate-specific antigen; PSMA: prostate-specific membrane antigen; PET: positron emission tomography; CT: computed tomography; OR: odd ratio; CI: confidence interval; Ref: reference.

salvage therapy, PSA persistence after primary therapy and disease progression before starting systemic therapies, respectively; $p<0.001)$. At multivariable analysis ISUP grade group, PSA level at ${ }^{68} \mathrm{Ga}$-PSMA PET/CT, PSA doubling time and the clinical setting were found to be independent predictors of positive ${ }^{68} \mathrm{Ga}$-PSMA PET/CT results (all $p \leq 0.04$ ) 
(Table III). A nomogram based on covariates included in the multivariate model demonstrated bootstrap-corrected predictive accuracy of $82 \%$ (Figure 2). Conclusion: Our nomogram could help physicians to select patients with different scenarios of recurrence who may benefit from ${ }^{68} \mathrm{Ga}$ PSMA PET/CT restaging.

\section{5 \\ MAGNETIC RESONANCE IMAGING AND ULTRASOUND FUSION BIOPSY IN FOLLOW-UP OF PATIENTS IN ACTIVE SURVEILLANCE PROTOCOL. CAN PSA DENSITY DISCRIMINATE PATIENTS AT HIGHER RISK OF RECLASSIFICATION?}

Giovanni La Croce ${ }^{1}$, Armando Stabile ${ }^{2}$, Giovanni Lughezzani ${ }^{3}$, Pietro Pepe ${ }^{4}$, Andrea Galosi ${ }^{5}$, Maria Nicolai ${ }^{1}$, Angelo Naselli ${ }^{6}$, Sandro Sironi ${ }^{7}$, Balzarini Luca ${ }^{8}$, Giorgio Guazzoni ${ }^{3}$, Alberto Briganti ${ }^{2}$, Francesco Montorsi ${ }^{2}$, Luigi Filippo Da Pozzo ${ }^{1}$ and Marco Roscigno ${ }^{1}$

${ }^{1}$ Department of Urology, ASST Papa Giovanni XXIII, Bicocca University, Milan, Italy; ${ }^{2}$ Department of Urology, San Raffaele Hospital, Vita Salute San Raffaele University, Milan, Italy; ${ }^{3}$ Department of Urology, Humanitas Rozzano Hospital, Humanitas University, Milan, Italy; ${ }^{4}$ Department of Urology, Cannizzaro

Hospital, Catania, Italy;

${ }^{5}$ Department of Urology, Ospedali Riuniti di Ancona, Marche Polytechnic University, Ancona, Italy;

${ }^{6}$ Department of Urology, San Giuseppe

Hospital, Milan, Italy;

${ }^{7}$ Department of Radiology, ASST Papa

Giovanni XXIII, Bicocca University, Milan, Italy;

${ }^{8}$ Department of Radiology, Humanitas Rozzano

Hospital, Humanitas University, Milan, Italy

Introduction and Objectives: Despite excellent oncological outcomes, patients in active surveillance (AS) will experience high risk of reclassification. MRI and ultrasound (US) fusion biopsy are increasingly used in the management of patients with clinically low-risk prostate cancer $(\mathrm{PCa})$, despite their role has not yet been established definitively. The aim of the study is to investigate the rate of reclassification in men with low-risk disease on AS, stratified on the basis of PI-RADS and PSA-density, with the aim to reduce potential unnecessary repeat biopsy procedures. Materials and Methods: We retrospectively evaluated $340 \mathrm{pts}$ undergoing confirmatory or follow-up biopsy according to PRIAS protocol, from January 2016 to September 2018. All patients were submitted to mpMRI on a $1.5 \mathrm{~T}$ or $3 \mathrm{~T}$ magnet, using

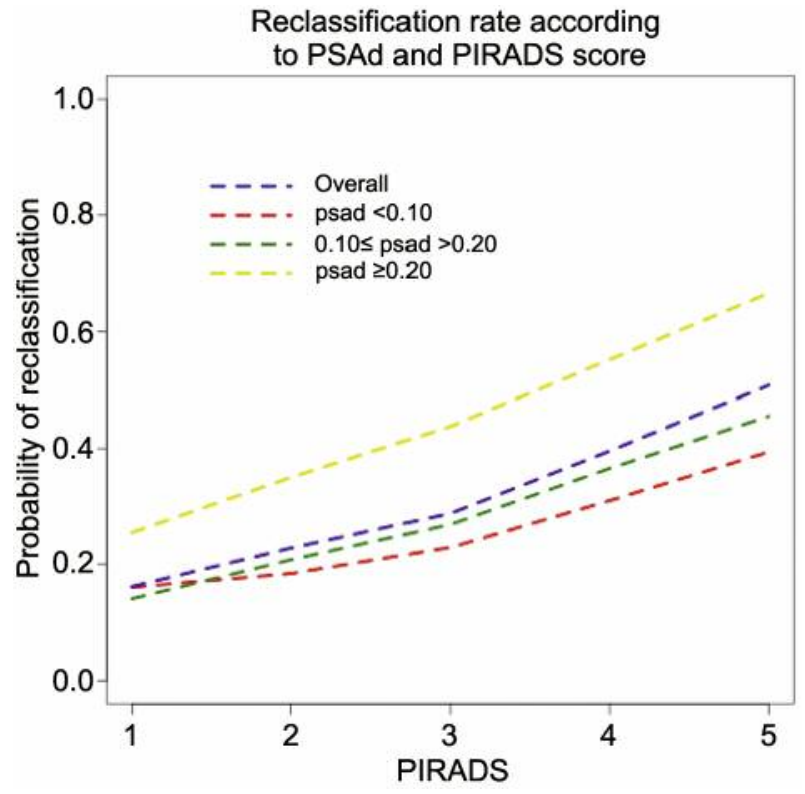

Figure 1. Reclassification rate according to PSAD and PIRADS score (Blue line: overall population; Red line: patients with PSAD $<0.10$; green line: patients with PSAD between 0.10 and 0.20; Yellow line: patients with PSAD >0.20).

triplanar high-resolution T2-w, axial DWI, and 3D T1-w dynamic contrast-enhanced sequences after injection of paramagnetic contrast agent. Pts with negative (-) mpMRI subsequently underwent systematic random biopsy. Pts with positive $(+)$ mpMRI (PI-RADS-V2 score $\geq 3$ ) underwent targeted fusion prostate biopsies ( 3 cores) + systematic random biopsies (12-18 cores). The primary objective of the study was the rate of reclassification, defined as the presence of clinically significant (cs)PCa with Gleason score $\geq 3+4$. Descriptive statistics and chi-square test were used to calculate differences between proportions. Different PSAD cut-off values were tested $(<0.10 ; 0.10-0.20 ; \geq 0.20)$. Multivariable logistic regression analyses (MVA) were used to predict the risk of overall reclassification during follow-up according to PSAD, after adjusting for covariates. Results: Median patient age and PSA was 67 years and $6.3 \mathrm{ng} / \mathrm{ml}$, respectively. Median PSA density was $0.12 \mathrm{ng} / \mathrm{ml} / \mathrm{cm}^{3}$. Median number of positive cores at initial biopsy was 1 (IQR:1,2). Eighty-four pts (24.7\%) had mpMRI(-); out of 256 pts with mpMRI (+), 71 (20.9\%) had PI-RADS 3, 146 (42.9\%) PI-RADS 4, and 39 (11.5\%) PI-RADS 5 lesions. At a median follow up of 12 months, 113 patients (33.2\%) were reclassified and switched to active treatment. In pts with mpMRI $(-)$ the rate of reclassification was $18 \%$. In mpMRI $(+)$, the overall rate of reclassification, at target + random biopsies, was $28 \%, 40 \%$ and $50 \%$ according to PIRADS 3, 4 and 5, respectively. When we stratified to PSAD, 
in case of $\mathrm{PSAD}<0.10$ the rate of reclassification was $16 \%$, $22 \%, 31 \%, 40 \%$ for mpMRI(-),PI-RADS 3,4 and 5 , respectively. In case of PSAD $\geq 0.20$ the rate of reclassification was $25 \%, 35 \%, 55 \%, 67 \%$ for mpMRI(-),PI-RADS 3, 4 and 5 , respectively (Figure 1). At MVA, PSAD $\geq 0.20(p=0.001$; $\mathrm{OR}=3.0)$, PI-RADS $5(p=0.02 ; \mathrm{OR}=3.43)$ were associated with the higher risk of reclassification, together with the number of positive cores at baseline biopsy $(p<0.001$; $\mathrm{OR}=2.27$ ). Conclusion: $\mathrm{PSAD} \geq 0.20$ may improve predictive accuracy of mpMRI results for reclassification of low-risk PCa pts in AS. PSAD $<0.10$ may help selection of pts at lower risk of harboring csPCa, in the PI-RADS 3,4 and 5 groups. However, it should be highlighted that the risk of reclassification is not negligible at any PSAD cut-off value, also in case of mpMRI(-).

\section{7 \\ DELAYING ADJUVANT CHEMOTHERAPY IN LOCALLY ADVANCED OR NODE-POSITIVE BLADDER CANCER MAY NOT AFFECT ONCOLOGIC OUTCOMES}

\author{
Matteo Soligo ${ }^{1}$, Valeria Lami $^{1}$, Luca Boeri ${ }^{2}$, \\ Alessandro Morlacco ${ }^{1}$, Vidtit Sharma ${ }^{2}$, Igor Frank ${ }^{2}$, \\ Steven Boorjian ${ }^{2}$ and R. Jeffrey Karnes ${ }^{2}$ \\ ${ }^{1}$ Urology Clinic, University of Padua, Padua, Italy; \\ ${ }^{2}$ Department of Urology, Mayo Clinic, \\ Rochester, MN, U.S.A.
}

Introduction and Objectives: Immediate adjuvant chemotherapy has been associated with better oncologic outcomes than deferred chemotherapy at relapse in pT3-4 and/or pN1-3 bladder cancer after radical cystectomy. However, the optimal timing for adjuvant chemotherapy has not yet been investigate. Therefore, we aimed to assess whether the recovery period from radical cystectomy to initiation of adjuvant chemotherapy may affect cancer-specific survival (CSS), overall survival (OS) and progression-free survival (PFS). Materials and Methods: Among 2000 M0 patients who underwent radical cystectomy for bladder cancer at a tertiary referral center between 1998-2017, we identified 708 subjects which had adjuvant chemotherapy for pT3-4 and/or N1-3 disease. Exclusion criteria were: incomplete demographic or clinical data; non-urothelial histology at radical cystectomy. 164 patients were included in the final analysis. Collected data included age, ECOG score, neoadjuvant chemotherapy regimens, adjuvant chemotherapy regimens, date of initiation and termination of adjuvant chemotherapy, pathologic data of radical cystectomy such as tumor and nodal stage, lymphovascular invasion and surgical margin status. Chemotherapy regimens were grouped as 1) gemcitabine-cisplatin (GC); 2) paclitaxel-gemcitabine-cisplatin
(PGC): 3) other chemotherapy regimens. Descriptive statistics were used to test the association between the recovery period and baseline demographic and clinical data. Kaplan-Meyer curves were used to compare CSS, OS and PFS according to the length of recovery period (weekly increments from 3 to 18). Multivariable Cox regression analysis were used to identify any predictors of CSS, OS and PFS. Results: The median follow-up time was 31.6 months. The median time to adjuvant chemotherapy initiation was 8.1 weeks. All-cause deaths, cancer-related deaths and recurrences were 103 $(62.8 \%), 86(52.4 \%)$ and $90(54.9 \%)$ respectively. Female gender and PGC ( $v s$. CG vs. other) were associated to an earlier initiation of adjuvant chemotherapy (6.28 vs. 8.43 weeks, $p=0.016 ; 7.1$ vs. 8.5 vs. 8.5 weeks, $p=0.038)$. Recovery period was not associated with CSS, OS or PFS. At multivariable analysis, older age $(\mathrm{HR}=1.02, p=0037)$, neoadjuvant chemotherapy regimens other than GC (6.38, $p<0.0001)$, pT3-4N1-3 (HR=1.9, $p=0.024)$ and non-cisplatinbased adjuvant chemotherapy regimens $(\mathrm{HR}=1.63, p=0.042)$ were associated to shorter OS. Neoadjuvant chemotherapy regimens other than GC (7.86, $p<0.0001)$, pT3-4N1-3 $(\mathrm{HR}=2.31, p=0.008)$ and non-cisplatin-based adjuvant chemotherapy regimens $(\mathrm{HR}=1.9, p=0.012)$ were associated to shorter CSS. No significant predictors of PFS was found. Conclusion: A recovery period ranging 3-18 weeks since cystectomy may not change significantly patients' prognosis. Non-cisplatin-based adjuvant chemotherapy should be avoided if patients are fit for cisplatin-based adjuvant chemotherapy.

48 ARE CAPSULAR BULGING AND
CONTACT'S EXTENSION PREDICTING
THE RISK OF EXTRAPROSTATIC
EXTENSION AT FINAL PATHOLOGY?

Matteo Manfredi ${ }^{1}$, Andrea Veltri $^{2}$, Agostino De Pascale $^{2}$, Stefano De Luca ${ }^{1}$, Enrico Checcucci ${ }^{1}$, Daniele Amparore ${ }^{1}$, Diletta Garrou ${ }^{1}$, Massimiliano Poggio ${ }^{1}$, Ivano Morra ${ }^{1}$, Giuseppe Stranieri², Giulia Gallo ${ }^{2}$, Michele Bini ${ }^{2}$, Dario Gned $^{2}$, Enrico Bollito ${ }^{3}$, Federica Massa ${ }^{3}$, Cristian Fiori ${ }^{1}$ and Francesco Porpiglia ${ }^{1}$

${ }^{1}$ Department of Urology, AOU San Luigi

Gonzaga, Orbassano, Italy;

${ }^{2}$ Department of Radiology, AOU San Luigi

Gonzaga, Orbassano, Italy;

${ }^{3}$ Department of Pathology, AOU San Luigi

Gonzaga, Orbassano, Italy

Introduction and Objectives: Over the last few decades, multiparametric Magnetic Resonance (mp-MRI) has become a fundamental investigation for pre-surgical assessment of prostate cancer $(\mathrm{PCa})$ and both the National Comprehensive 
Cancer Network (NCCN) and the European Association of Urology (EAU) recommend its use. Not only it allows to guide target biopsies, but its role is crucial for localization, risk stratification and staging of the tumor. Its importance is even more relevant when it comes to the definition of capsular bulging and extraprostatic extension (EPE), elements that were rarely described with previous imaging techniques. EPE is defined as the extension of tumor beyond the confines of the gland into the periprostatic soft tissue; furthermore, it is a well-recognized adverse prognostic factor resulting in upstaging of prostatic carcinoma. Preoperative identification of EPE is decisive for a correct management of the disease: it affects how the nerve sparing technique is performed. In presence of capsular bulging at mp-MRI, a correlation has been noticed between the maximum length of capsular contact (LCCmax) and EPE at final pathological findings. The aim of our study was to assess the capacity of capsular bulging and LCCmax to predict EPE. Materials and Methods: We analyzed retrospectively our prospective maintained robot-assisted radical prostatectomy database and extracted the patients who were submitted to mp-MRI preoperatively from January 2016 to December 2017. The mpMRI of the prostate was performed on a 1.5-T MR scanner, using a two-channel endorectal coil. Just before the beginning of the examination, $1 \mathrm{~mL}$ butylscopolamine bromide was injected i.v. to reduce bowel movements. The balloon of the endorectal coil was distended with $\sim 60 \mathrm{~mL}$ air, in order to block the coil in the correct position. All robotassisted radical prostatectomy procedures were performed by a single highly experienced surgeon. In the first place, we chose a cut-off of LCCmax $>20 \mathrm{~mm}$ to predict EPE, according to other widely accepted scientific articles from Literature. Secondly, we evaluated if $15 \mathrm{~mm}$ of contact extension were sufficient to predict EPE. The role of capsular bulging itself was then analyzed. The presence of EPE at final pathological findings was defined by a single, highly experienced, uropathologist. At last, we calculated positive and negative predictive values (PPV and NPV) of these different variables. Results: 162 patients were included in our study. EPE was observed in 97 patients at the final histopathological findings. $71(73.2 \%)$ patients had a LCCmax $\geq 20 \mathrm{~mm}$ (PPV: 0.82; NPV: 0.77$)$ at mp-MRI. Concerning our second objective, considering $15 \mathrm{~mm}$ as cut-off, $87 / 97(89.7 \%)$ patients had LCCmax $\geq 15 \mathrm{~mm}$ (PPV: 0.77; NPV: 0.79). At last, 84/97 $(86.59 \%)$ patients showed capsular bulging at mp-MRI (PPV: 0.79; NPV: 0.77). Discussion and Conclusion: Literature data suggest that the extent of EPE on the radical prostatectomy specimen may be a means of identifying higher-risk of recurrence and progression, as well as an increased risk of prostate cancer-related mortality. Tailoring the surgical approach in terms of nerve sparing robot-assisted radical prostatectomy has represented, over the last years, the most important challenge in radical prostatectomy: it requires an experienced urologist to balance the risk of EPE, with a correlated risk of positive margins, versus the advantage of neurovascular preservation. According to our study both LCCmax and capsular bulging at pre-surgical mp-MRI are reasonable predictors of the risk of EPE at final pathology. This findings at imaging are reproducible and accurate signs of EPE. As mentioned previously, the identification of EPE is crucial for the preoperative evaluation of prostate tumor. This will define surgeon's approach towards peri-capsular structures, in particular posterolateral neurovascular bundles, with clinical implications in postoperative urinary continence and erectile function. Further studies are required to corroborate the results.

\section{9 \\ HOW DOES ${ }^{68}$ GA-PSMA PET/CT IMPACT THE TREATMENT MANAGEMENT IN PATIENTS WITH PROSTATE CANCER RECURRENCE AFTER SURGERY?}

Lorenzo Bianchi ${ }^{1}$, Marco Borghesi ${ }^{1}$, Umberto Barbaresi ${ }^{1}$, Federico Mineo Bianchi ${ }^{1}$, Andrea Angiolini ${ }^{1}$, Paolo Castellucci ${ }^{2}$, Francesco Ceci ${ }^{2}$, Francesco Chessa ${ }^{1}$, Carlo Casablanca ${ }^{1}$, Carlo Beretta ${ }^{1}$, Cristian Vincenzo Pultrone ${ }^{1}$, Hussam Dababneh ${ }^{1}$, Cristina Nanni ${ }^{2}$, Riccardo Schiavina ${ }^{1}$, Stefano Fanti ${ }^{1}$ and Eugenio Brunocilla ${ }^{1}$

${ }^{1}$ Division of Urology, Sant'Orsola-Malpighi Hospital, University of Bologna, Bologna, Italy; ${ }^{2}$ Division of Nuclear Medicine, Sant'Orsola-Malpighi Hospital, University of Bologna, Bologna, Italy

Introduction: To evaluate the clinical impact of ${ }^{68} \mathrm{Ga}$ Prostate-specific membrane antigen (PSMA) Positron Emission Tomography/Computed tomography (PET/CT) on the planned management in prostate cancer ( $\mathrm{PCa}$ ) patients with biochemical recurrence (BCR) after surgery. Materials and Methods: We enrolled 276 patients with BCR after surgery. To assess the site of recurrence each patient was referred to ${ }^{68} \mathrm{Ga}$-PSMA PET/CT. Moreover, 110 and 90 men were re-staged with ${ }^{11} \mathrm{C}$-Choline PET/CT and conventional imaging (including computed tomography and/or magnetic resonance imaging and/or bone scan) at last 6 months before ${ }^{68} \mathrm{Ga}$-PSMA PET/CT, respectively. The intended treatment was retrospectively determined by a multidisciplinary team (Prostate Cancer Unit Board) blinded to imaging findings, based on EAU guidelines, patient clinical condition and clinical parameters. The reassessment of treatment plan was recorded by the same board after revision of ${ }^{68} \mathrm{Ga}$-PSMA PET/CT $(\mathrm{n}=274),{ }^{11} \mathrm{C}$ Choline PET/CT $(n=110)$ and conventional imaging $(n=90)$, blindly to each other. The effective clinical impact of ${ }^{68} \mathrm{Ga}$ - 
Table I. Overall patient characteristics.

\begin{tabular}{|c|c|c|c|c|c|c|c|c|c|}
\hline Variable & $\begin{array}{l}\text { Overall } \\
\text { PET/CT } \\
\text { negative }\end{array}$ & $\begin{array}{c}\text { PSMA } \\
\text { PET/CT } \\
\text { positive }\end{array}$ & PSMA & $p$-Value & Variable & $\begin{array}{l}\text { Overall } \\
\text { PET/CT } \\
\text { negative }\end{array}$ & $\begin{array}{c}\text { PSMA } \\
\text { PET/CT } \\
\text { positive }\end{array}$ & PSMA & $p$-Value \\
\hline $\begin{array}{l}\text { No. of patients }(\%) \\
\text { Age (years) }\end{array}$ & $276(100.0)$ & $145(52.5)$ & $131(47.5)$ & - & $\begin{array}{l}\text { Adjuvant } \\
\text { radiotherapy, n (\%) }\end{array}$ & & & & \\
\hline Median & 68 & 68 & 68 & 0.4 & No & $203(73.6)$ & $114(78.6)$ & $89(67.9)$ & 0.04 \\
\hline IQR & $62-73$ & $62-73$ & $63-73$ & & Yes & $73(26.4)$ & $31(21.4)$ & $42(32.1)$ & \\
\hline $\begin{array}{l}\text { Preoperative } \\
\text { PSA }(\mathrm{ng} / \mathrm{ml})\end{array}$ & & & & & $\begin{array}{l}\text { Adjuvant RT } \\
\text { site, } \mathrm{n}(\%)\end{array}$ & & & & \\
\hline Median & 8.5 & 8.1 & 8.9 & 0.4 & Pelvis & $36(49.3)$ & $12(38.7)$ & $24(57.1)$ & 0.1 \\
\hline IQR & $6.0-12.0$ & $6.2-12.0$ & $6.0-12.1$ & & Pelvis+PLN & $37(50.7)$ & $19(61.3)$ & $18(42.9)$ & \\
\hline Date of surgery, & & & & & Adjuvant ADT, n (\%) & & & & \\
\hline $\mathrm{n}(\%)$ & & & & & No & $231(83.7)$ & $128(88.3)$ & $103(78.6)$ & 0.03 \\
\hline$<2000$ & $25(9.1)$ & $16(11.0)$ & $9(6.9)$ & 0.4 & Yes & $45(16.3)$ & $17(11.7)$ & $28(21.4)$ & \\
\hline $2000-2005$ & $61(22.1)$ & $31(21.4)$ & $30(22.9)$ & & PSA at BCR $(\mathrm{ng} / \mathrm{ml})$ & & & & \\
\hline 2006-2010 & $144(52.2)$ & $71(49.0)$ & $73(55.7)$ & & Median & 0.72 & 0.52 & 1.1 & $<0.001$ \\
\hline$>2010$ & $46(16.7)$ & $27(18.6)$ & $19(14.5)$ & & IQR & $0.37-1.40$ & $0.30-0.96$ & $0.5-2.0$ & \\
\hline $\begin{array}{l}\text { Pathologic gleason } \\
\text { score, } \mathrm{n}(\%)\end{array}$ & & & & & $\begin{array}{l}\text { PSA ranges at } \\
\text { BCR, n }(\%)\end{array}$ & & & & \\
\hline 6 & $20(7.2)$ & $13(9.0)$ & $7(85.3)$ & 0.37 & $0.2-0.5$ & $100(36.2)$ & $70(48.3)$ & $30(22.9)$ & $<0.001$ \\
\hline 7 & $169(61.2)$ & $92(63.4)$ & $77(58.8)$ & & $0.5-1$ & $71(25.7)$ & $41(28.3)$ & $30(22.9)$ & \\
\hline $8-10$ & 87 (31.6) & $40(27.6)$ & 47 (35.9) & & $1-2$ & $64(23.2)$ & $24(16.5)$ & $40(30.5)$ & \\
\hline Pathologic stage, & & & & & $>2$ & $41(14.9)$ & $10(6.9)$ & $31(23.7)$ & \\
\hline $\begin{array}{l}\mathrm{n}(\%) \\
\text { Missing data }\end{array}$ & $9(3.3)$ & $7(4.8)$ & $2(1.5)$ & 0.009 & $\begin{array}{l}\text { Time to BCR } \\
\text { (months) }\end{array}$ & & & & \\
\hline pT2 & $98(35.5)$ & $57(39.3)$ & $41(31.3)$ & & Median & 26 & 27 & 26 & 0.6 \\
\hline pT3a & $95(34.4)$ & $54(37.2)$ & $41(31.3)$ & & IQR & $12-50$ & $14-51$ & $12-50$ & \\
\hline pT3b-pT4 & $74(26.8)$ & $27(18.5)$ & $47(35.9$ & & Conventional & & & & \\
\hline PLND, n (\%) & & & & & imaging, n (\%) & & & & \\
\hline Missing data & $8(2.9)$ & $4(2.8)$ & $4(3.1)$ & 0.6 & No & $122(44.2)$ & $73(50.3)$ & $49(37.4)$ & 0.05 \\
\hline No & $67(24.3)$ & $41(28.3)$ & $26(19.8)$ & & Choline-PET CT & $110(39.9)$ & $48(33.1)$ & $62(47.3)$ & \\
\hline Yes & $201(72.8)$ & $100(69.0)$ & $101(77.1)$ & & CT scan & $28(10.1)$ & $9(6.2)$ & $19(14.5)$ & \\
\hline Nodal status, n (\%) & & & & & MRI & $55(19.9)$ & $29(20.0)$ & $26(19.8)$ & \\
\hline pNO & $164(81.6)$ & $87(87.0)$ & $77(76.2)$ & 0.5 & Bone scan & $37(13.4)$ & $16(11.0)$ & $21(16.0)$ & \\
\hline
\end{tabular}

IQR: Interquartile range; PSA: prostate specific antigen; PLND: pelvic lymph nodes dissection; RT: radiotherapy; ADT: androgen deprivation therapy; BCR: biochemical relapse; CT: computerized tomography; MRI: magnetic resonance imaging.

PSMA PET/CT, 11C-Choline PET/CT and conventional imaging was rated as major (change in therapeutic approach), minor (same treatment, but modified therapeutic strategy) or none. Results: Table I shows patients' characteristics after stratifying patients according to ${ }^{68} \mathrm{GaPSMA}-\mathrm{PET} / \mathrm{CT}$ results (positive $v s$. negative). The overall detection rate of PSMA PET/CT was $47.5 \%$. Overall median time to BCR was $26(\mathrm{IQR}=12-50)$ months and median PSA at PSMA PET/CT was $0.71 \mathrm{ng} / \mathrm{ml}$ (IQR=0.37-1.40). Patients with positive finding at PSMA PET/CT had significantly higher pathologic stage and PSA at BCR and received more often adjuvant therapies, related to men with negative scan (all $p \leq 0.04$ ). Table II describes

differences in clinical management of patients before and after revision of each imaging technique. Table III describes the clinical impact of PSMA PET/CT, Choline $\mathrm{PET} / \mathrm{CT}$ and Conventional imaging. ${ }^{68} \mathrm{Ga}$-PSMA PET/CT led to a major treatment change in 177 cases $(64.1 \%)$ with a minor clinical impact of 2.5\%.11C-Choline PET/CT and conventional imaging had a major clinical impact of $36.4 \%$ and $34.4 \%$, with a minor clinical impact of $15.5 \%$ and 23.3\%, respectively. Conclusion: 68Ga-PSMA PET/CT revealed a significant clinical impact in restaging $\mathrm{PCa}$ patients, since it allows to radically change the intended treatment approach before imaging evaluation, in roughly two out three individuals. 
Table II. Prostate cancer patients' management after imaging assessment: ${ }^{68} \mathrm{Ga}$-PSMA PET/CT (n=276 patients), ${ }^{11} \mathrm{C}$-Choline PET/CT ( $n=110$ patients) and conventional imaging ( $n=90$ patients).

\begin{tabular}{|c|c|c|c|c|}
\hline & $\begin{array}{c}\text { Clinical management } \\
\text { before imaging }\end{array}$ & $\begin{array}{c}{ }^{68} \mathrm{Ga}-\mathrm{PSMA} \\
\mathrm{PET} / \mathrm{CT}\end{array}$ & $\begin{array}{l}\text { Choline } \\
\text { PET/CT* }^{*}\end{array}$ & $\begin{array}{l}\text { Conventional imaging } \\
(\mathrm{CT} / \mathrm{MRI} / \mathrm{BS})^{\#}\end{array}$ \\
\hline No treatment, $\mathrm{n}(\%)$ & $31(11.2)$ & $99(35.9)$ & $30(27.3)$ & $24(26.7)$ \\
\hline Salvage RT, n (\%) & $105(38.1)$ & $72(26.0)$ & $29(26.4)$ & $32(35.6)$ \\
\hline prostatic bed & $0(0)$ & $23(8.3)$ & $8(7.3)$ & $21(23.3)$ \\
\hline prostatic bed + pelvic nodes & $102(37)$ & $19(6.9)$ & $16(14.6)$ & $10(11.1)$ \\
\hline pelvic nodes & $3(1.1)$ & $12(4.3)$ & $2(1.8)$ & $1(0.01)$ \\
\hline extrapelvic nodes & $0(0)$ & $15(5.4)$ & $1(0.9)$ & $0(0)$ \\
\hline bones & $0(0)$ & $3(1.1)$ & $2(1.8)$ & $0(0)$ \\
\hline visceral & $0(0)$ & $0(0)$ & $0(0)$ & $0(0)$ \\
\hline Salvage lymph node dissection, $\mathrm{n}(\%)$ & $0(0)$ & $9(3.3)$ & $2(1.8)$ & $0(0)$ \\
\hline Surgical metastasis resection, $\mathrm{n}(\%)$ & $0(0)$ & $1(0.4)$ & $0(0)$ & $0(0)$ \\
\hline Systemic therapies, n (\%) & $140(50.7)$ & $95(34.4)$ & $49(44.5)$ & $34(37.8)$ \\
\hline
\end{tabular}

PSMA PET/CT: Prostate specific membrane antigen Positron Emission Tomography; CT: computed tomography; MRI: magnetic resonance imaging; BS: bone scan. *Patients referred to PSMA PET/CT and choline PET/CT $(\mathrm{n}=110)$. "Patients referred to PSMA PET/CT and conventional imaging (including CT and/or MRI and/or BS; $\mathrm{n}=90$ ).

Table III. Clinical impact of different imaging techniques (namely, ${ }^{68} \mathrm{Ga}$-PSMA PET/CT in 276 patients, ${ }^{11}$ C-Choline PET/CT in 110 patients and conventional imaging in 90 patients) on treatment changes.

\begin{tabular}{|c|c|c|c|}
\hline & $\begin{array}{c}{ }^{68} \mathrm{Ga}-\mathrm{PSMA} \\
\mathrm{PET} / \mathrm{CT}\end{array}$ & $\begin{array}{l}{ }^{11} \text { C-Choline } \\
\text { PET/CT* }\end{array}$ & $\begin{array}{c}\text { Conventional imaging } \\
(\mathrm{CE}-\mathrm{CT} / \mathrm{MRI} / \mathrm{BS})^{\#}\end{array}$ \\
\hline Major clinical impact & $177(64.1)$ & $40(36.4)$ & $31(34.4)$ \\
\hline 1. From palliative to curative & $49(17.8)$ & $11(10)$ & $6(6.7)$ \\
\hline 2. From curative to palliative & $20(7.2)$ & $2(1.8)$ & $3(3.3)$ \\
\hline 3. From palliative to surveillance & $22(8)$ & $8(7.3)$ & $10(11.1)$ \\
\hline 4. From curative to surveillance & $66(23.9)$ & $16(14.5)$ & $7(7.8)$ \\
\hline 5. From surveillance to curative & $14(5.1)$ & $3(2.7)$ & $5(5.6)$ \\
\hline 6. From surveillance to palliative & $6(2.2)$ & $0(0)$ & $0(0)$ \\
\hline Minor clinical impact & $7(2.5)$ & $17(15.5)$ & $21(23.3)$ \\
\hline more aggressive/extended treatment & $4(1.4)$ & $17(15.5)$ & $6(6.7)$ \\
\hline less aggressive/extended treatment & $3(1)$ & $0(0)$ & $15(16.7)$ \\
\hline No treatment change & $92(33.3)$ & $53(48.2)$ & $38(42.2)$ \\
\hline
\end{tabular}

PSMA PET/CT: Prostate specific membrane antigen Positron Emission Tomography; CT: computed tomography; MRI: magnetic resonance imaging; BS: bone scan. *Patients referred to PSMA PET/CT and choline PET/CT $(n=110)$. " Patients referred to PSMA PET/CT and conventional imaging (including CT and/or MRI and/or BS; $\mathrm{n}=90$ ).

50

\section{MAGNETIC RESONANCE IMAGING ALONE SHOULD NOT BE CONSIDERED AS A STAND- ALONE TEST FOR DISEASE RECLASSIFICATION OF MEN IN ACTIVE SURVEILLANCE}

Marco Roscigno $^{1}$, Armando Stabile ${ }^{2}$, Giovanni Lughezzani ${ }^{3}$, Giovanni La Croce ${ }^{1}$, Pietro Pepe ${ }^{4}$, Maria Nicolai ${ }^{1}$, Andrea Galosi ${ }^{5}$, Angelo Naselli ${ }^{6}$, Luca Balzarini ${ }^{7}$, Sandro Sironi ${ }^{8}$, Giorgio Guazzoni ${ }^{3}$, Alberto Briganti ${ }^{2}$, Francesco Montorsi ${ }^{2}$ and Luigi Filippo Da Pozzo ${ }^{1}$
${ }^{1}$ Department of Urology, ASST Papa Giovanni XXIII, Bicocca University, Milan, Italy ${ }^{2}$ Department of Urology, San Raffaele Hospital, Vita Salute San Raffaele University, Milan, Italy; ${ }^{3}$ Department of Urology, Humanitas Rozzano Hospital, Humanitas University, Milan, Italy; ${ }^{4}$ Department of Urology, Cannizzaro

Hospital, Catania, Italy;

${ }^{5}$ Department of Urology, Ospedali Riuniti

Di Ancona, Marche Polytechnic

University, Ancona, Italy; 


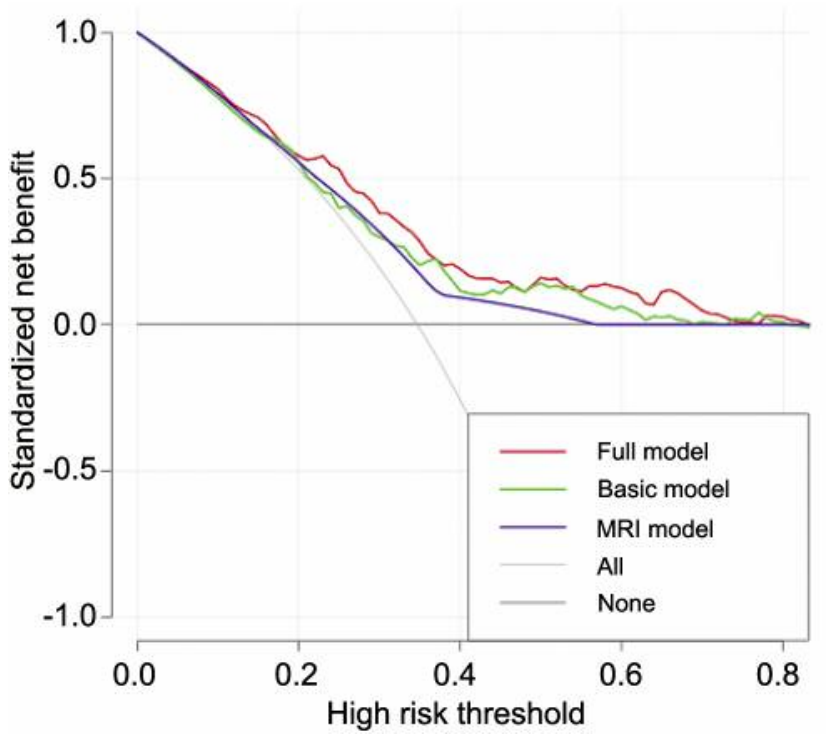

Figure 1. Clinical net benefit deriving from the use of the three evaluated models.

\author{
${ }^{6}$ Department of Urology, San Giuseppe \\ Hospital, Milan, Italy; \\ ${ }^{7}$ Department of Radiology, Humanitas Rozzano \\ Hospital, Humanitas University, Milan, Italy; \\ ${ }^{8}$ Department of Radiology, ASST Papa Giovanni \\ XXIII, Bicocca University, Milan, Italy
}

Introduction and Objectives: Multiparametric magnetic resonance imaging (mpMRI) and ultrasound (US) fusion biopsy are increasingly used in the management of patients with clinically low-risk prostate cancer ( $\mathrm{PCa})$, despite their role has not yet been established definitively. The aim of the study is to evaluate whether mpMRI alone could be used as a stand-alone test suggesting risk of reclassification in men in active surveillance (AS). Materials and Methods: We retrospectively evaluated 340 pts undergoing confirmatory or follow-up biopsy according to PRIAS protocol, from January 2016 to September 2018. All patients were submitted to mpMRI on a $1.5 \mathrm{~T}$ or $3 \mathrm{~T}$ magnet, using triplanar high-resolution T2-w, axial DWI, and 3D T1-w dynamic contrast-enhanced sequences after injection of paramagnetic contrast agent. Pts with negative (-) mpMRI subsequently underwent systematic random biopsy. Pts with positive $(+)$ mpMRI (PI-RADS-V2 score $\geq 3$ ) underwent targeted fusion prostate biopsies (3 cores) + systematic random biopsies (1218 cores). Multivariate logistic regression analyses (MVA) was used to create three models predicting the probability of disease reclassification (defined as presence of $\mathrm{PCa} G \mathrm{GS} \geq 3+4$ at prostate biopsy): a basic model including only clinical variables (age, PSAD and number of positive cores at baseline); an MRI model including only PI-RADS score; a full model including both the previous ones. The predictive accuracy (PA) of each model was quantified using the AUC. The clinical net benefit deriving from the use of each model was assessed with the use of decision curve analysis. Results: Median patient age and PSA was 67 years and $6.3 \mathrm{ng} / \mathrm{ml}$, respectively. Median PSA density was $0.12 \mathrm{ng} / \mathrm{ml} / \mathrm{cm}^{3}$. Median number of positive cores at initial biopsy was 1 (IQR:1,2). Eighty-four pts $(24.7 \%)$ had mpMRI (-); out of 256 pts with mpMRI (+), 71 (20.9\%) had PI-RADS 3, 146 (42.9\%) PI-RADS 4, and 39 (11.5\%) PI-RADS 5 lesions. At a median follow up of 12 months, 113 patients (33.2\%) were reclassified and switched to active treatment. In pts with mpMRI (-) the rate of reclassification was $18 \%$. In mpMRI $(+)$, the overall rate of reclassification, at target + random biopsies, was $28 \%, 40 \%$ and $50 \%$ according to PI-RADS 3 , 4 and 5 , respectively. In the basic model, PSAD and the number of positive cores at baseline biopsy were independent predictors of risk of reclassification $(p=0.001$; $\mathrm{OR}=66.4$ and $p<0.001 ; \mathrm{OR}=2.2$, respectively), with a PA of $69 \%$. In the MRI model, PI-RADS 5 was predictor of reclassification $(p=0.002 ; \mathrm{OR}=4.76)$ and the PA was lower than in the basic model (AUC 62\%). The full model, that includes clinical variables and MRI results, had the best PA of $72 \%$. PSAD $(p=0.01 ; \mathrm{OR}=28.6)$, number of positive cores at baseline $(p<0.001 ; \mathrm{OR}=2.20)$ and PI-RADS $5(p=0.02 ; \mathrm{OR}=3.6)$ were independent predictors of reclassification. Figure 1 depicts the clinical net benefit deriving from the use of the three evaluated models. Conclusion: MRI alone should not be used in clinical practice as a stand-alone trigger for disease reclassification. The combination of MRI and other clinical variables still represents the most accurate approach to patients on AS.

\section{2 CONCORDANCE BETWEEN INDEX LESION AND SIDE OF LYMPH NODE INVASION IN PATIENTS SUBTENDED TO EXTENDED LYMPHATIC DISSECTION}

Camilla Capretti, Lorenzo Montesi, Luca Leone, Erika Palagonia, Alessio Papaveri, Aldo Parlavecchio, Carmine Franzese, Lucio Dell'Atti and Andrea Benedetto Galosi

Clinic of Urology, AOU Polytechnic of Marche Region, Torrette, Italy

Introduction: We evaluated the concordance between laterality of index lesion and lymph node involvement (LI) in patients underwent extended pelvic lymphadenectomy (ePLND) during radical prostatectomy for prostate cancer.

Materials and Methods: We evaluated 264 patients who 
underwent retropubic and robot-assisted radical prostatectomy from January 2016 to February 2018. Patients were divided according D'Amico classification group risk. We calculated Briganti and MSKCC nomograms for LI risk. We considered the laterality of index lesion, detected with mpMRI and biopsy, the laterality of lymphadenectomy (unilateral or bilateral), the number of lymph nodes removed and the laterality of LI. Results: 181 patients underwent ePLND, unilaterally in 56 cases $(30.9 \%)$ and bilaterally in 125 cases $(69.1 \%)$. 10,91 (DS=7.41) lymph nodes were removed in case of unilateral ePLND and 16.55 (DS 9.12) in case of bilateral ePLND. Lymph nodes resulted positive for cancer invasion in 22 cases: 20 in bilateral ePLND group (16\%) and 2 in unilateral ePLND group (3.6\%). Lymph nodes resulted positive in the right side in 10 cases; in this group, the index lesion was in the right lobe in 6 cases, in the left lobe in 2, bilateral in 1 and anterior in 1 . Lymph nodes resulted positive in the left side in 7 cases; in this group, the index lesion was in the right lobe in 3 cases, in the left in 2, bilateral in 2. Lymph nodes resulted positive bilaterally in 5 cases; in this group the index lesion was in the left lobe in 2 cases, bilateral in $3(p=0.21)$. We observed discordance of the laterality between the index lesion and positive lymph nodes in 5 cases; 2 cases were of Prognostic Grade Group (PGG) 3 and 3 cases PGG5. According to D'Amico classification group risk, LI resulted in 2 low risk patients $(2.4 \%)$, in 10 intermediate risk patients $(7.4 \%)$, in 11 high risk patients $(24.4 \%)$. In low-risk patients, LI resulted ipsilateral to index lesion. Among patients with intermediate risk and LI, in 2 cases (both PGG3) positive lymph nodes resulted contralateral to index lesion. Conclusion: We observed discordance between the laterality of index lesion and the lymph node involvement in 5 out of 181 patients $(2.6 \%)$ who underwent ePLND; all cases were of intermediate and high risk according to D'Amico classification group risk. Our data support bilateral ePLND in patients with intermediate and high-risk tumor.

\section{3 \\ WHICH PATIENTS WITH CLINICALLY \\ LOCALIZED RENAL TUMOUR WOULD \\ ACHIEVE THE TRIFECTA AFTER PARTIAL NEPHRECTOMY?}

Riccardo Schiavina, Marco Borghesi, Lorenzo Bianchi, Pietro Piazza, Matteo Droghetti, Umberto Barbaresi,

Francesco Chessa, Federico Mineo Bianchi,

Carlo Beretta, Carlo Casablanca, Andrea Angiolini,

Serena Di Fresco, Cristian Vincenzo Pultrone and

Eugenio Brunocilla

Division of Urology, Sant'Orsola-Malpighi Hospital, University of Bologna, Bologna, Italy

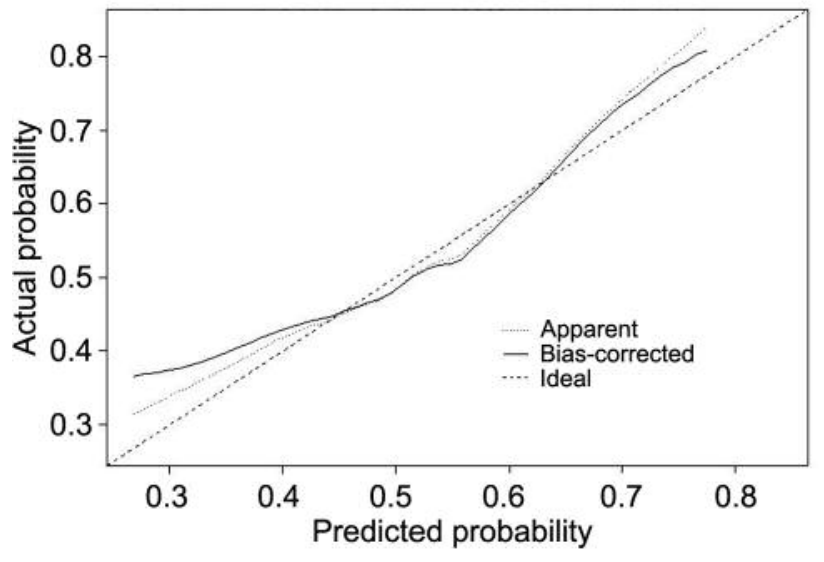

Figure 1. Nomogram predicting the likelyhood to achieve the Trifecta rate after partial nephrectomy.

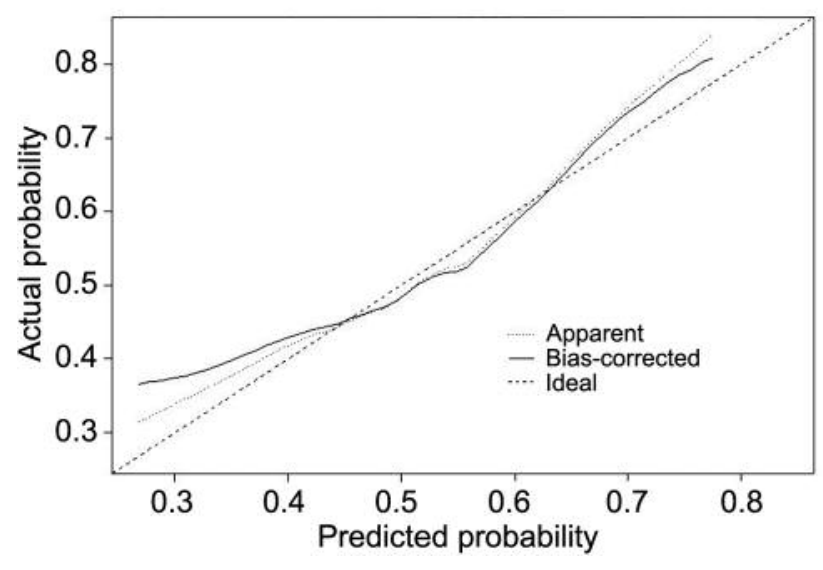

Figure 2. Bootstrap-corrected predictive accuracy of the nomogram.

Introduction and Objectives: To develop a clinical nomogram aimed to predict the achievement of Trifecta in patients treated with open, laparoscopic and robotic partial nephrectomy (PN) for localized renal masses $(\leq \mathrm{cT} 2)$. Materials and Methods: We retrospectively evaluated 482 consecutive patients who underwent open (OPN: 243), laparoscopic (LPN: 156) and robotic (RAPN: 83) partial nephrectomy (PN) for clinically localized renal cell carcinoma (RCC) at single tertiary center between March 2000 and September 2018. Trifecta was defined as the achievement of warm ischemia time (WIT) $\leq 20 \mathrm{~min}$, absence of positive surgical margins (PSM) and absence of any kind of postoperative complication. First, we compared clinical, pathologic and perioperative outcomes within the three surgical approaches. Second, multivariate logistic regression was performed to identify which co-variates [including American Society of Anesthesiologists (ASA) 
Table I. Preoperative patients characteristics after stratifying according to surgical technique (OPN: open partial nephrectomy; LPN: laparoscopic partial nephrectomy; RAPN: robot-assisted partial nephrectomy).

\begin{tabular}{|c|c|c|c|c|c|}
\hline Variable & Overall & OPN & LPN & RAPN & $p$-Value \\
\hline No. of patients, $\mathrm{n}(\%)$ & $482(100)$ & $243(50.4)$ & $156(32.4)$ & $83(17.2)$ & - \\
\hline \multicolumn{6}{|l|}{ Age (years) } \\
\hline Median (IQR) & $64(53-71)$ & $63(53-71)$ & $64(55-71)$ & $64(53-71)$ & 0.9 \\
\hline \multicolumn{6}{|l|}{ Gender, n (\%) } \\
\hline Male & $317(65.8)$ & $161(66.3)$ & $100(64.1)$ & $56(67.5)$ & \multirow[t]{2}{*}{0.9} \\
\hline Female & $165(34.2)$ & $82(33.7)$ & $56(35.9)$ & $27(32.5)$ & \\
\hline \multicolumn{6}{|l|}{ BMI $\left(\mathrm{kg} / \mathrm{m}^{2}\right)$} \\
\hline Median (IQR) & $26(24-27)$ & $26(24-29)$ & $27(25-31)$ & $26(24-30)$ & 0.6 \\
\hline \multicolumn{6}{|l|}{ ASA score, n (\%) } \\
\hline 1 & $37(7.7)$ & $18(7.4)$ & $98(5.8)$ & $10(12)$ & \multirow[t]{4}{*}{0.4} \\
\hline 2 & $273(56.6)$ & $141(58)$ & $87(55.8)$ & $45(54.2)$ & \\
\hline 3 & $162(33.6)$ & $77(31.7)$ & $57(36.5)$ & $28(33.7)$ & \\
\hline 4 & $10(2.1)$ & $7(2.9)$ & $3(1.9)$ & $0(0)$ & \\
\hline \multicolumn{6}{|l|}{ Tumor side, n (\%) } \\
\hline Right & $253(52.5)$ & $134(55.1)$ & $75(48.1)$ & $44(53)$ & \multirow[t]{3}{*}{0.3} \\
\hline Left & $218(45.2)$ & $101(41.6)$ & $79(50.6)$ & $38(45.8)$ & \\
\hline Bilateral & $11(2.3)$ & $8(3.3)$ & $2(1.3)$ & $1(1.2)$ & \\
\hline \multicolumn{6}{|l|}{ Preoperative $\mathrm{Hb}(\mathrm{g} / \mathrm{dL})$} \\
\hline Median (IQR) & $14.4(13.4-15.3)$ & $14.1(13.2-15.1)$ & $14.5(13.5-15.4)$ & $14.5(13.3-15.3)$ & 0.07 \\
\hline \multicolumn{6}{|c|}{ Preoperative Creatinine (mg/dl) } \\
\hline Median (IQR) & $0.9(0.8-1.10)$ & $0.9(0.8-1.12)$ & $0.89(0.8-1.10)$ & $0.9(0.8-1.10)$ & 0.3 \\
\hline \multicolumn{6}{|c|}{ Preoperative eGFR $\left(\mathrm{ml} / \mathrm{min} / 1,73 \mathrm{~m}^{2}\right)$} \\
\hline Median (IQR) & $84(66-108)$ & $81(62-106)$ & $87(69-110)$ & $88(70-110)$ & 0.08 \\
\hline \multicolumn{6}{|l|}{ Clinical stage, $\mathrm{n}(\%)$} \\
\hline cT1a & $407(84.4)$ & $204(84)$ & $138(88.5)$ & $65(78.3)$ & \multirow[t]{2}{*}{0.1} \\
\hline $\mathrm{cT} 1 \mathrm{~b}$ & $75(15.6)$ & $39(16)$ & $18(11.5)$ & $18(21.7)$ & \\
\hline \multicolumn{6}{|l|}{ Clinical tumor size $(\mathrm{cm})$} \\
\hline Median (IQR) & $3(2-3.7)$ & $3(2.3-3.8)$ & $2.5(1.9-3.3)$ & $2.6(2-3.9)$ & 0.06 \\
\hline \multicolumn{6}{|l|}{ PADUA score, $\mathrm{n}(\%)$} \\
\hline $6-7$ & $243(50.4)$ & $114(46.9)$ & $92(59)$ & $37(44.6)$ & \multirow[t]{3}{*}{0.1} \\
\hline $8-9$ & $182(37.8)$ & $96(39.5)$ & $51(32.7)$ & $35(42.2)$ & \\
\hline $10-14$ & $57(11.8)$ & $33(13.6)$ & $13(8.3)$ & $11(13.3)$ & \\
\hline \multicolumn{6}{|l|}{ R.E.N.A.L. score, n (\%) } \\
\hline $4-6$ & $257(53.3)$ & $106(43.6)$ & 107 (68.6) & $44(53)$ & \multirow[t]{3}{*}{$<0.001$} \\
\hline $7-9$ & $213(44.2)$ & $134(55.1)$ & $45(28.8)$ & $34(41)$ & \\
\hline $10-12$ & $12(2.5)$ & $3(1.2)$ & $4(2.6)$ & $5(6)$ & \\
\hline \multicolumn{6}{|l|}{ Surgery date, n (\%) } \\
\hline $2000-2005$ & $27(5.6)$ & $24(9.9)$ & $3(1.9)$ & $0(0)$ & \multirow[t]{4}{*}{$<0.001$} \\
\hline $2006-2010$ & $99(20.5)$ & $79(32.5)$ & $20(12.8)$ & $0(0)$ & \\
\hline $2011-2015$ & $264(54.8)$ & $129(53.1)$ & $112(71.8)$ & $23(27.7)$ & \\
\hline $2016-2017$ & $92(19.1)$ & $11(4.5)$ & $21(13.5)$ & $60(72.3)$ & \\
\hline
\end{tabular}

OPN: Open partial nephrectomy; LPN: laparoscopic partial nephrectomy; RAPN: robot-assisted partial nephrectomy; BMI: body-mass index; Hb: hemoglobin; ASA: American Society of Anaesthesiologists; eGFR: estimated glomerular filtration rate; CKD: chronic kidney disease.

score, clinical size of the tumor, urinary collecting system (UCS) involvement and surgical approach] independently predict the Trifecta achievement. Finally, regression-based coefficients were used to develop a nomogram predicting the likelihood of achieving Trifecta and 200 bootstrap resamples were used for internal validation. Results: The three cohorts were comparable in terms of demographics and clinical characteristics (Table I). WIT $\leq 20$ min was achieved in 211 (86.8\%), $122(78.2 \%)$ and $73(88 \%)$ patients undergoing OPN, LPN and RAPN, respectively $(p<0.001)$. Positive surgical margins rate was $11.5 \%$, $16.7 \%$ and $3.6 \%$ after OPN, LPN and RAPN, respectively $(p=0.01)$. Postoperative complications rate was significantly higher in patients treated with OPN (36.8\%) 
Table II. Intra and postoperative patients characteristics after stratifying according to surgical technique (OPN: open partial nephrectomy; LPN: laparoscopic partial nephrectomy; RAPN: robot-assisted partial nephrectomy).

\begin{tabular}{|c|c|c|c|c|c|}
\hline Variable & Overall & OPN & LPN & RAPN & $p$-Value \\
\hline \multicolumn{6}{|l|}{ WIT, n (\%) } \\
\hline 0 & $169(35.1)$ & $68(28)$ & $83(53.2)$ & $18(21.7)$ & \multirow[t]{3}{*}{$<0.001$} \\
\hline $1-19$ & $237(49.2)$ & $143(58.8)$ & $39(25)$ & $55(66.3)$ & \\
\hline$\geq 20$ & $76(15.8)$ & $32(13.2)$ & $34(21.8)$ & $10(12)$ & \\
\hline \multicolumn{6}{|l|}{ WIT, min* } \\
\hline Median (IQR) & $15(10-19)$ & $14(9-17)$ & $19(14-25)$ & $14(10-17)$ & $<0.001$ \\
\hline \multicolumn{6}{|l|}{ Operative time ( $\mathrm{min})$} \\
\hline Median (IQR) & $131(102-180)$ & $110(93-136)$ & $139(105-185)$ & $225(190-265)$ & $<0.001$ \\
\hline \multicolumn{5}{|l|}{ Delta eGFR $24 \mathrm{~h}$ after surgery } & 0.004 \\
\hline \multicolumn{6}{|c|}{ Intra-operative complications, $\mathrm{n}(\%)$} \\
\hline No & $439(91.1)$ & $213(87.7)$ & $151(96.8)$ & $75(90.4)$ & \multirow[t]{2}{*}{0.007} \\
\hline Yes & $43(8.9)$ & $30(12.3)$ & $5(3.2)$ & $8(9.6)$ & \\
\hline \multicolumn{6}{|c|}{ Global post-operative complications, n (\%) } \\
\hline No & $338(70.1)$ & $154(63.4)$ & $120(76.9)$ & $64(77.1)$ & \multirow[t]{2}{*}{0.005} \\
\hline Yes & $144(29.9)$ & $89(36.8)$ & $36(23.1)$ & $19(22.9)$ & \\
\hline \multicolumn{6}{|l|}{$\begin{array}{l}\text { Post-operative complications } \\
\text { grade, } \mathrm{n}(\%)^{\#}\end{array}$} \\
\hline Clavien 1-2 & $112(77.8)$ & $71(79.8)$ & $26(72.2)$ & $15(78.9)$ & \multirow[t]{2}{*}{0.6} \\
\hline Clavien $\geq 3$ & $32(22.2)$ & $18(20.2)$ & $10(27.8)$ & $32(22.2)$ & \\
\hline \multicolumn{6}{|l|}{ Medical post-operative } \\
\hline complications, n (\%) & $43(8.9)$ & $25(10.3)$ & $9(5.8)$ & $9(10.8)$ & 0.2 \\
\hline \multicolumn{6}{|l|}{ Surgical post-operative } \\
\hline \multicolumn{6}{|c|}{ Surgical complications grade, $\mathrm{n}(\%)^{\#}$} \\
\hline Clavien 1-2 & $77(76.2)$ & $52(81.3)$ & $18(66.7)$ & $7(70)$ & \multirow[t]{2}{*}{0.3} \\
\hline Clavien $\geq 3$ & $24(23.8)$ & $12(18.8)$ & $9(33.3)$ & $3(30)$ & \\
\hline \multicolumn{6}{|l|}{ Positive surgical margins, $\mathrm{n}(\%)$} \\
\hline No & $425(88.2)$ & $215(88.5)$ & $130(83.3)$ & $80(96.4)$ & \multirow[t]{2}{*}{0.01} \\
\hline Yes & $57(11.8)$ & $28(11.5)$ & $26(16.7)$ & $3(3.6)$ & \\
\hline \multicolumn{6}{|l|}{ Length of stay (days) } \\
\hline Median (IQR) & $5(4-6)$ & $5(4.8-6)$ & $4(3-5)$ & $4(3-5)$ & $<0.001$ \\
\hline \multicolumn{6}{|l|}{ Trifecta rate, n (\%) } \\
\hline No & $226(46.9)$ & $124(51)$ & $77(49.4)$ & $25(30.1)$ & \multirow[t]{2}{*}{0.003} \\
\hline Yes & $256(53.1)$ & $119(49)$ & $79(50.6)$ & $58(69.9)$ & \\
\hline \multicolumn{6}{|l|}{ Pathological Tumor Size $(\mathrm{cm})$} \\
\hline Median (IQR) & $3(2-3.8)$ & $3(2.3-4)$ & $2.7(2-3.5)$ & $3.2(2-4.1)$ & 0.009 \\
\hline \multicolumn{6}{|l|}{ Histology, n (\%) } \\
\hline Oncocytoma & $84(17.4)$ & $44(18.1)$ & $30(19.2)$ & $10(12)$ & 0.2 \\
\hline Angiomyolipoma & $25(5.2)$ & $15(6.2)$ & $8(5.1)$ & $2(2.4)$ & \\
\hline Other benign renal tumours & $18(3.7)$ & $9(3.7)$ & $8(5.1)$ & $1(1.2)$ & \\
\hline Clear-cell renal carcinoma & $228(47.3)$ & $114(46.9)$ & $68(43.6)$ & $46(55.4)$ & \\
\hline Papillary renal carcinoma & $83(17.2)$ & 34 (14) & $31(19.9)$ & $18(21.7)$ & \\
\hline Chromophobe carcinoma & $35(7.3)$ & $22(9.1)$ & $7(4.5)$ & $6(7.2)$ & \\
\hline Other maligne renal tumours & $9(1.9)$ & $5(2.1)$ & $4(2.6)$ & $0(0)$ & \\
\hline Pathological tumor stage, n (\%) & & & & & \\
\hline pT1a & $298(80.7)$ & $194(79.8)$ & $137(87.8)$ & $58(69.9)$ & 0.04 \\
\hline pT1b & $64(13.3)$ & $36(14.8)$ & $12(7.7)$ & $16(19.3)$ & \\
\hline pT2a & $6(1.2)$ & $3(1.2)$ & $1(0.6)$ & $2(2.4)$ & \\
\hline pT2b & $0(0)$ & $0(0)$ & $0(0)$ & $0(0)$ & \\
\hline pT3a & $23(4.8)$ & $10(4.1)$ & $6(3.8)$ & $7(8.4)$ & \\
\hline
\end{tabular}

WIT: Warm Ischemia Time; OPN: open partial nephrectomy; LPN: laparoscopic partial nephrectomy; RAPN: robot-assisted partial nephrectomy. *only considering patients whom underwent surgery with warm ischemia; ${ }^{*}$ only considering patients who developed postoperative complications. 
Table III. Univariable and multivariable logistic regression to predict the Trifecta achievement (negative surgical margins, absence of postoperative complications and warm ischemia time $<20$ minutes).

\begin{tabular}{|c|c|c|c|c|}
\hline \multirow[b]{2}{*}{ Variables } & \multicolumn{2}{|c|}{ Univariable analysis } & \multicolumn{2}{|c|}{ Multivariable analysis } \\
\hline & OR (95\% C.I.) & $p$-Value & OR (95\% C.I.) & $p$-Value \\
\hline Age (years) & $1.01(0.99-1.02)$ & 0.3 & - & - \\
\hline \multicolumn{5}{|l|}{ ASA score } \\
\hline $1-2$ & 1.0 & (Ref) & & 2.0 (Ref) \\
\hline $3-4$ & $0.64(0.44-0.93)$ & 0.02 & $0.63(0.43-0.93)$ & 0.02 \\
\hline Preoperative creatinine level mg/dl (continuous variable) & $0.92(0.57-1.49)$ & 0.7 & - & - \\
\hline BMI (continuous variable) & $0.99(0.96-1.04)$ & 0.9 & - & - \\
\hline Gender & & & - & - \\
\hline Male & $1.0($ Ref $)$ & & & \\
\hline Female & $1.13(0.78-1.65)$ & 0.5 & & \\
\hline Padua score & & & - & - \\
\hline $6-7$ & 1.0 (Ref) & 0.1 & & \\
\hline $8-9$ & $0.93(0.63-1.37)$ & 0.7 & & \\
\hline $10-14$ & $0.54(0.30-0.97)$ & 0.04 & & \\
\hline R.E.N.A.L. score & & & - & - \\
\hline $4-6$ & $1.0(\operatorname{Ref})$ & 0.3 & & \\
\hline $7-9$ & $0.75(0.52-1.08)$ & 0.1 & & \\
\hline $10-12$ & $0.77(0.24-2.46)$ & 0.7 & & \\
\hline Polar localization & & & - & - \\
\hline Superior-inferior pole & 1.0 (Ref) & 0.9 & & \\
\hline Parenchimal & $0.98(0.68-1.41)$ & & & \\
\hline Tumor location & & & - & - \\
\hline Lateral & $1.0(\operatorname{Ref})$ & 0.7 & & \\
\hline Medial & $0.93(0.64-1.36)$ & & & \\
\hline Renal sinus invasion & & & - & - \\
\hline No & 1.0 (Ref) & 0.2 & & \\
\hline Yes & $0.72(0.45-1.14)$ & & & \\
\hline Tumor growth pattern & & & - & - \\
\hline$\geq 50 \%$ exophytic & 1.0 (Ref) & & & \\
\hline$<50 \%$ exophytic & $0.73(0.50-1.06)$ & 0.9 & & \\
\hline Endophytic & $0.97(0.44-2.16)$ & 0.9 & & \\
\hline Clinical tumor size $(\mathrm{cm})$ & $0.83(0.72-0.96)$ & 0.01 & $0.86(0.74-1.01)$ & 0.07 \\
\hline \multicolumn{5}{|l|}{ Urinary collecting system } \\
\hline Not involved & $1.0(\operatorname{Ref})$ & 0.02 & $1.0(\operatorname{Ref})$ & 0.02 \\
\hline Dislocated/infiltrated & $0.56(0.35-0.90)$ & & $0.56(0.33-0.93)$ & \\
\hline \multicolumn{5}{|l|}{ Surgical technique } \\
\hline RARP & $1.0(\operatorname{Ref})$ & & 1.0 (Ref) & \\
\hline LPN & $0.44(0.25-0.78)$ & 0.005 & $0.39(0.23-0.68)$ & 0.001 \\
\hline OPN & $0.41(0.24-0.70)$ & 0.001 & $0.38(0.22-0.69)$ & 0.001 \\
\hline
\end{tabular}

OR: Odds Ratio; CI: confidence interval; ASA: American Society of Anaesthesiologists score; OPN: open partial nephrectomy; LPN: laparoscopic partial nephrectomy; RAPN: robot assisted partial nephrectomy.

compared to LPN (23.1\%) and RAPN (22.9\%; $p=0.005$; Table II). Trifecta has been achieved in $49 \%, 40.6 \%$ and $69.9 \%$ of patients undergoing OPN, LPN and RAPN, respectively $(p=0.003)$. At multivariate analyses, ASA score [odd ratio (OR): 0.70], UCS involvement $(\mathrm{OR}=0.56)$ and robotic approach $(\mathrm{OR}=2.48)$ were independent predictors of Trifecta achievement (all $p \leq 0.03$; Table III). A nomogram based on covariates included in the multivariate model demonstrated bootstrap-corrected predictive accuracy of $62 \%$. (Figures 1 and 2). Conclusion: RAPN allowed to obtain higher rates of Trifecta achievement in patients treated with PN. ASA score, UCS involvement and the surgical technique were independent predictors of Trifecta achievement. Our nomogram could facilitate the preoperative counselling as individualized prediction of surgical outcomes. 
54

\section{HISTOLOGICAL CHARACTERIZATION OF LYMPH NODES METASTASIS IN MIXED UROTHELIAL-SQUAMOUS HISTOLOGICAL VARIANT AT RADICAL CYSTECTOMY}

Giovanni La Croce, Michele Manica, Paolo Barzaghi, Federico Pellucchi, Lorenzo Rocchini, Antonino Saccà, Diego Angiolilli, Richard Lawrence, John Naspro and Luigi Filippo Da Pozzo

Department of Urology, Bicocca University, ASST Papa Giovanni XXIII, Milan, Italy

Introduction: Bladder urothelial histological variants (BHV) at final pathological report after radical cystectomy (RC) are a frequent event, about $20 \%$ of cases. Moreover, as reported in literature, histological variants are more aggressive diseases, with a high rate of lymph node invasion (LNI). Indeed about $45 \%$ of patients with BHV have one LNI compared to the $35 \%$ of pure urothelial variants. No data are available in literature about the characterization of lymph node histology in BHV. The aim of our study was to investigate the presence or not of histological variant into the lymph node in $\mathrm{N}+$ patients and evaluate if oncological outcomes are affected by the node histology. Patients and Methods: We evaluated 391 consecutive non-metastatic patients with $\mathrm{BC}$, treated with RC at a single tertiary referral centre between 2008 and 2015. In the study we considered patients with BHV diagnosed with lymph node invasion at final pathological report, $34(8.6 \%)$ patients. Specimens were evaluated by a dedicated uro-pathologist. Descriptive statistic was used to identify characteristics of the population. Univariable and multivariable Cox proportional hazards regression analyses model was used to predict cancer specific mortality (CSM), overall mortality (OM) and recurrence rate. Covariates included age at surgery, gender, pathological $\mathrm{T}$ stage, pathological $\mathrm{N}$ stage, pathological grade, surgical margins and lymph vascular invasion (LVI). Results: Median age was 72 years (IQR=50-84 years). 25 patients $(73 \%)$ were male. Overall, $20(59 \%), 8(23 \%)$ and $30(88 \%)$ patients were recorded with LVI, positive surgical margins and pT3-4 stage, respectively. Median number of lymph nodes removed were 24 (10-63) while median number of positive nodes was 1 (1-29). The median percentage of histological variant was $50 \%(\mathrm{IQR}=5-90 \%)$. Overall CSM was observed in $28(82 \%)$ patients, whereas OM was observed in 33 patients $(97 \%)$ and recurrence in $28(82 \%)$ patients. Median follow-up was 16 months (2-84). At the final pathological report $8(23.5 \%)$ patients had squamocellular variant in lymph nodes metastasis while 26 $(76.5 \%)$ had pure urothelial lymph node metastasis. Bladder percentage of histological variant is not associated with the presence of histological variant in lymph nodes metastasis $(p=0.9)$. Moreover, both at univariate and multivariate analysis histological variant in lymph node metastasis was not associated with higher risk of CSM, recurrence or OM (respectively $p=0.5, p=0.9$ and $p=0.5$ ) Predictors of CSM were nodal stage, $\mathrm{T}$ stage, grade and LVI. While predictors of recurrence were $\mathrm{T}$ stage and LVI. Finally, the predictor of $\mathrm{OM}$ was age at surgery. Conclusion: Literature data about the frequency of histological variants at RC are confirmed in our study. Moreover, we show for the first time the histological characterization of lymph node metastasis in BHV. Furthermore, bladder percentage of histological variant is not associated with the presence of variant in node metastasis. Finally, the presence of histological variant in lymph node metastasis does not affect survival outcomes.

\section{5}

\section{ADJUVANT CHEMOTHERAPY FOLLOWING RADICAL CYSTECTOMY: PROPENSITY-SCORE MATCHED EVALUATION OF SURVIVAL OUTCOMES}

$\underline{\text { Matteo Soligo }^{1}, \text { Marialaura Righetto }^{1} \text {, Alessandro Morlacco }}{ }^{1}$, Michele Colicchia ${ }^{1}$, Luca Boeri ${ }^{2}$, Vidit Sharma ${ }^{2}$, Igor Frank ${ }^{2}$ and R. Jeffrey Karnes ${ }^{2}$

${ }^{1}$ Urology Clinic, University of Padua, Padua, Italy;

${ }^{2}$ Department of Urology, Mayo Clinic,

Rochester, MN, U.S.A.

Introduction and Objectives: Adjuvant chemotherapy is recommended in pT3-4 and/or pN1-3 bladder cancer following radical cystectomy. However, its benefit on survival outcomes is still controversial. Therefore, the aims of the present study were: to investigate whether adjuvant chemotherapy may improve survival in pT3-4 and/or pN1-3 bladder cancer; to identify the most effective AC regimens and any predictors of cancer-specific survival (CSS), overall survival (OS) and progression-free survival. Materials and Methods: Among $2200 \mathrm{cM} 0$ patients who underwent radical cystectomy in a tertiary referral center between 1998-2017, we identified 996 patients with pT3-4 and/or N1-3 disease. Exclusion criteria were: incomplete demographic or clinical data; non-urothelial histology at radical cystectomy. Patients were grouped as follow: 1) optimal adjuvant chemotherapy (OAC): cisplatin-based adjuvant chemotherapy for $>3$ cycles; 2) suboptimal adjuvant chemotherapy (SAC): cisplatin-based adjuvant chemotherapy for $<3$ cycles or noncisplatin-based adjuvant chemotherapy; 3) no adjuvant chemotherapy. Propensity score matching by age, sex, neoadjuvant chemotherapy, pathologic tumor stage, pathologic nodal stage, eGFR and post-operative ECOG selected 92, 53 and 104 patients respectively. Descriptive statistics were used to show baseline demographic and 


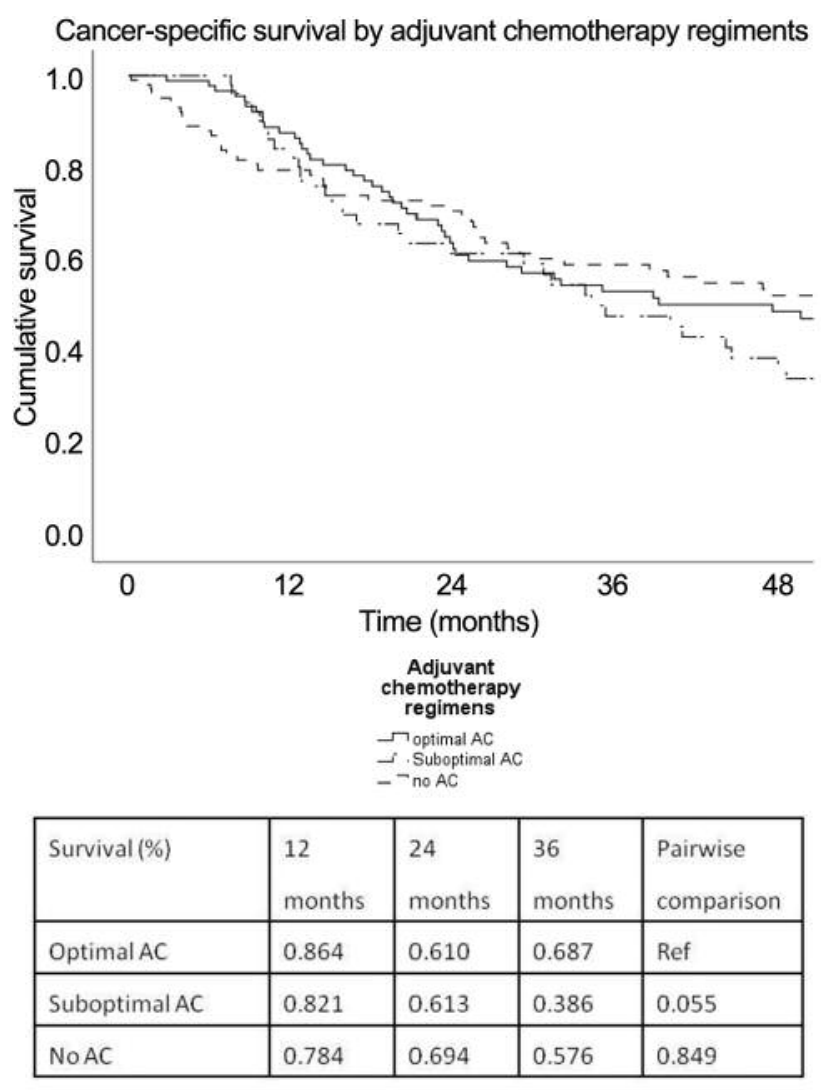

Figure 1. Kaplan-Meier curve showing cancer-specific survival by adjuvant chemotherapy (AC) regimens. Cumulative survivals at fixed time intervals are reported in the table below the graphic.

clinical data. Kaplan-Meyer curves and log-rank tests were used to compare CSS and OS between AC regimens. Multivariable Cox regression analysis was used to identify any predictors of CSS and OS. Results: Matching variables were balanced between groups, except for positive pathologic node status (OAC vs. SAC vs. no AC: 62, 67.4\% vs. $38,71.7 \%$ vs. $32(30.8 \%)$ and post-operative eGFR (67.6 vs. 58.5 vs. $66.3 \mathrm{ml} / \mathrm{min}, p=0.021)$. No significant difference was found in the median time to recurrence (OAC vs. SAC vs. no AC: 9.7 vs. 11.2 vs. 11.1 months, $p=0.828)$, median follow-up time (26.1 vs. 29.2 vs. 30.3 months, $p=0.49)$ and all-cause deaths $(58,63 \%$ vs. $40,75.5 \%$ vs. $72,69.2 \%$, $p=0.29)$. Cancer-specific deaths were higher in the SAC group $(46,50 \%$ vs. 37, $63.8 \%$ vs. $52,50 \%, p=0.037)$. Kaplan-Meier curves did not demonstrate an association between OAC, SAC or no AC and OS, CSS, PFS (Figures 1,2 and 3 ). At multivariable analysis, no significant predictor of PFS was found. Higher age at $\mathrm{RC}(\mathrm{HR}=1.03$, $p=0.005)$, neoadjuvant chemotherapy (HR 2.1, $p=0.007)$, pT3-4 $(\mathrm{HR}=2.72, p<0.0001), \mathrm{pN} 1-3(\mathrm{HR}=1.97, p=0.001)$, lymphovascular invasion (HR 1.57, p.022) were associated

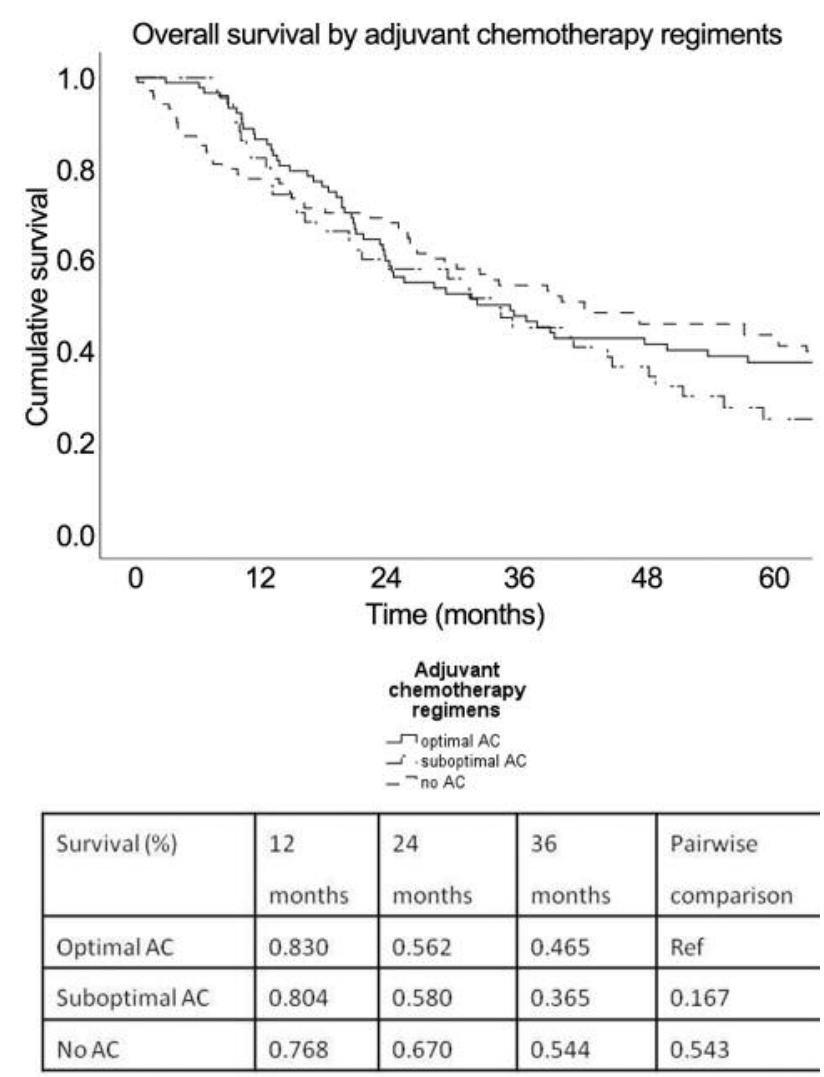

Figure 2. Kaplan-Meier curve showing overall survival by adjuvant chemotherapy $(A C)$ regimens. Cumulative survivals at fixed time intervals are reported in the table below the graphic.

to shorter CSS. Only OAC ( $\mathrm{HR}=0.61, p=0.022)$ was significantly associated with longer CSS. Conclusion: Despite the poor prognosis associated to pT3-4 and/or pN13 disease, optimal adjuvant chemotherapy (OAC) may improve survival outcomes following $\mathrm{RC}$ in selected patients, though it may not affect progression.

\section{6 \\ SYSTEMATIC URETERAL FROZEN SECTIONS DURING RADICAL CYSTECTOMY FOR BLADDER CANCER. IS IT REALLY USEFUL?}

Giovanni La Croce, Michele Manica, Paolo Barzaghi, Federico Pellucchi, Lorenzo Rocchini, Antonino Saccà, Diego Angiolilli, Richard Lawrence, John Naspro and Luigi Filippo Da Pozzo

Department of Urology, Bicocca University, ASST Papa Giovanni XXIII, Milan, Italy

Introduction: In our center, distal ureteral frozen sections 


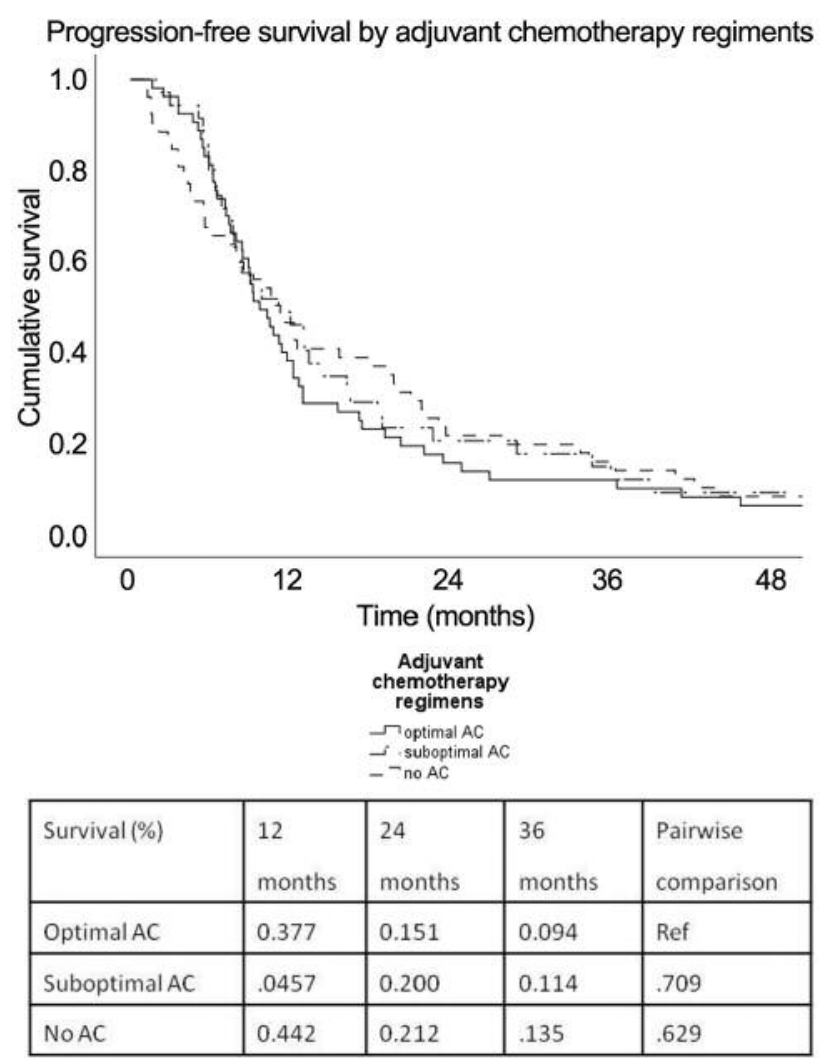

Figure 3. Kaplan-Meier curve showing progression-free survival by adjuvant chemotherapy (AC) regimens. Cumulative survivals at fixed time intervals are reported in the table below the graphic.

during radical cystectomy (RC) due to bladder cancer (BC) are routinely performed and repeated, if positive, until the reach of tumour negative samples. However, despite several studies, there is not a global consensus on the adequate management of positive margins. Especially it is not clear if the positive findings are correlated with oncological outcomes. Aim of our study was to evaluate if the presence of positive ureteral frozen sections (negative at final pathological report) impacts on recurrence and survival outcomes. Patients and Methods: We evaluated 391 consecutive nonmetastatic patients diagnosed with $\mathrm{BC}$ and treated with $\mathrm{RC}$ at a single tertiary referral center between 2008 and 2015. We systematically performed ureteral frozen section independently to the bladder diversion or to TNM status. Univariable and multivariable Cox proportional hazards regression analyses model was used to predict cancer specific mortality (CSM), overall mortality $(\mathrm{OM})$ and recurrence. The Kaplan-Meier method was used to compare recurrence, CSM and OM in the overall population. Covariates included age at surgery, gender, pathological $\mathrm{T}$ stage, pathological $\mathrm{N}$ stage, pathological grade, surgical margins and lymph vascular invasion. Results: Median age was 71 years (IQR $=40-90$ years). 322 patients (82\%) were male. Overall, 117 (29.9\%), 51(13\%) and $174(44.5 \%)$ patients were recorded with lymph node metastases, positive surgical margins and pT3-4 stage, respectively. CSM was observed in 146 (37\%) patients, whereas OM was observed in 194 patients (49\%) and recurrence in 154 (39\%) patients. Median follow-up was 30 months (IQR=5-109 months). Intraoperative frozen sections were positive in $62(16 \%)$ patients. At univariate analysis presence of positive frozen section was associated with higher risk of CSM and recurrence (respectively $\mathrm{HR}=1.82$ and 1.98; $\mathrm{CI}=1.15-2.84$ and 1.12-2.92; all $p<0.001)$ but not $\mathrm{OM}(p=0.3)$. At multivariate analysis presence of positive frozen sections was associated with higher recurrence and CSM risk, (respectively $\mathrm{HR}=1.58$ and 1.52; $\mathrm{CI}=1.05-2.37$ and 1.06-2.42; $p<0.001$ and $p=0.04)$ conversely any association was found with $\mathrm{OM}$ $(\mathrm{HR}=1.15 ; p=0.5)$. Other predictors of CSM were nodal stage, T stage, grade and lymph vascular invasion. While other predictors of recurrence were $\mathrm{T}$ stage and nodal stage. Finally, predictors of OM were age at surgery, $\mathrm{T}$ stage and nodal stage. Conclusion: Ureteral frozen sections should be always performed. Considering the impact on oncological outcomes, positive ureteral frozen sections, even when negative at the final report, should be taken into account in the planning of the follow-up of patients who underwent $\mathrm{RC}$ due to $\mathrm{BC}$.

\section{7}

\section{CONCOMITANT CARCINOMA IN SITU AT RADICAL CYSTECTOMY: SURVIVAL, RECURRENCE AND FOLLOW-UP IMPLICATIONS}

Giovanni La Croce, Michele Manica, Federico Pellucchi, Lorenzo Rocchini, Paolo Barzaghi, Antonino Saccà,

Diego Angiolilli, Richard Lawrence, John Naspro and Luigi Filippo Da Pozzo

Department of Urology, Bicocca University, ASST Papa Giovanni XXIII, Milan, Italy

Introduction: The presence of carcinoma in situ (CIS) at transurethral resection increases the risk of progression to invasive disease and recurrence. However, the evidence about the presence of concomitant CIS on survival outcomes after radical cystectomy (RC) due to bladder cancer (BC) is poor. The aim of our study is to evaluate if the presence of concomitant CIS at RC impacts on recurrence and survival outcomes. Patients and Methods: We evaluated 391 consecutive nonmetastatic patients diagnosed with $\mathrm{BC}$ and treated with $\mathrm{RC}$ at a single tertiary referral center between 2008 and 2015. Univariable and multivariable Cox 
proportional hazards regression analyses model was used to predict cancer specific mortality (CSM), overall mortality $(\mathrm{OM})$ and recurrence. The Kaplan-Meier method was used to compare recurrence, CSM and $\mathrm{OM}$ in the overall population and in pT0-pT2 and pT3-pT4 patients stratified according to the presence of CIS. Covariates included age at surgery, gender, pathological $\mathrm{T}$ stage, pathological $\mathrm{N}$ stage, pathological grade, surgical margins and lymph vascular invasion. Results: Median age was 71 years (IQR $=40-90$ years). 322 patients (82\%) were male. Overall, 117 (29.9\%), $51(13 \%)$ and $174(44.5 \%)$ patients were recorded with lymph node metastases, positive surgical margins and pT3-4 stage, respectively. CSM was observed in 146 (37\%) patients, whereas OM was observed in 194 patients (49\%) and recurrence in 154 (39\%) patients. Median follow-up was 30 months (IQR=5-109 months). CIS was associated to pT0-2 in $144(36 \%)$ patients and to pT3-4 in $86(21 \%)$ patients. At univariate analysis concomitant CIS was associated with higher risk of CSM, OM and recurrence (all $p<0.001$ ). However, the presence of CIS was not associated with any survival effect or recurrence at MVA Cox regression analyses (all $p=0.3$ ). Similar data were reported if patients were stratified according to pathological T stage. Indeed, at MVA Cox regression analyses, CIS did not condition survival outcomes or recurrence free survival neither in pT0-pT2 patients (all $p \leq 0.04$ ) neither in $\mathrm{p} \mathrm{T3}-\mathrm{pT} 4$ patients (all $\mathrm{p} \geq 0.2$ ); Predictors of CSM were nodal stage, T stage, lymph vascular invasion and positive margins, while predictors of $\mathrm{OM}$ were age at surgery, nodal stage and T stage. Conclusion: CIS is considered as negative prognostic factor at TURV that contributes to cystectomy indication. However, concomitant presence of CIS at cystectomy does not increase the risk of recurrence and does not impact on survival outcomes. The most important features to consider in follow-up schedules are pathological stage, lymph node status and lymph vascular invasion.

\section{8 \\ COMPARISON UPGRADING OF GLEASON SCORE 3+4 CANCERS AT RADICAL PROSTATECTOMY IN TARGET BIOPSY VERSUS STANDARD BIOPSY}

Stefano De Luca ${ }^{1}$, Cristian Fiori ${ }^{1}$, Ivano Morra ${ }^{1}$, Matteo Manfredi ${ }^{1}$, Daniele Amparore ${ }^{1}$, Diletta Garrou ${ }^{1}$, Dario Peretti ${ }^{1}$, Enrico Checcucci ${ }^{1}$, Roberta Aimar ${ }^{1}$, Marco Cossu ${ }^{1}$, Francesca Ragni ${ }^{1}$, Massimiliano Poggio ${ }^{1}$, Razvan Gabriel Niculescu ${ }^{1}$, Federica Massa ${ }^{2}$, Enrico Bollito ${ }^{2}$ and Francesco Porpiglia ${ }^{1}$

${ }^{1}$ Department of Urology, AOU San Luigi

Gonzaga, Orbassano, Italy;

${ }^{2}$ Department of Pathology, AOU San Luigi

Gonzaga, Orbassano, Italy
Introduction and Objectives: Gleason score (GS) assignment is crucial, being one of the most important pretreatment criteria for patient counseling and decision-making process. Many reports have demonstrated that cancers with greater amounts of Gleason pattern (GP) 4 and 5 tissues have a worse prognosis. Recent studies demonstrated that multiparametric Magnetic Resonance (mpMRI)/TRUS fusion biopsy (target biopsy, TBx) ensured a higher prostate cancer (PCa) detection accuracy, a better performance in discriminating significant PCa from not significant ones, when compared to "untargeted" standard biopsy ( $\mathrm{SBx})$, reducing the risk of GS upgrading at RP. The aim of our study was to evaluate the GS $3+4$ upgrading rates in the biopsy versus final pathology after surgery in patients who underwent fusion targeted biopsy comparing untargeted standard biopsy. We also evaluated the impact of percent pattern 4 and cribriform glands at biopsy in the risk of GS $3+4=7$ prostate cancer $(\mathrm{PCa})$ upgrading at radical prostatectomy (RP). Materials and Methods: We sequentially prospectively enrolled 223 patients with GS $3+4$ PCa on repeat biopsy (122 SBx and $101 \mathrm{TBx}$ ) who underwent laparoscopic robot-assisted RP. All patients had negative digital rectal examination. GS evaluation was performed according to 2016 WHO- International Society of Urological Pathology (ISUP) grade group system. One of the main changes in this modified system, was that some cribriform cancer glands originally scored as GP 3 have now been recategorized as GP 4. Cribriform foci were assessed as Gleason pattern (GP) 4, because cribriform morphology is associated with a worse prognosis. The number of cores with cancer foci and histopathologic type of GP 4 were recorded. Whole-mount histological sections resected from the RP formalin-fixed specimens were used as reference standards. Clinically significant PCa was defined as a tumour of $>0.5 \mathrm{ml}$ and/or pathological GS $>6$; consequently, PCa foci with a volume $<0.5 \mathrm{ml}$ and GS $<6$ were defined as clinically not significant. Results: No significant differences were found when comparing the two groups ( $\mathrm{SBx}$ and $\mathrm{TBx}$ ) for median prostate volume $(p=0.901)$ while the median tumor volume was larger in the SBx cohort (3.9vs. $2.7 \mathrm{ml}, p=0.010$ ). Of the overall GS of $3+4$ biopsies, 137 (61.4\%) and $86(38.6 \%)$ showed GP 4 in $<11 \%$ and in $11-49 \%$ of the tissue, respectively. Minimal GP 4 cancers had a significant lower median volume $(1.3 \mathrm{ml})$ than those with $\mathrm{GP} 4 \geq 11 \%$ ( $2.3 \mathrm{ml})$, $(p<0.001)$. A pathological GS $3+4$ was confirmed for $59.8 \%$ SBx patients, while the same Gleason sum was confirmed for $81.2 \%$ TBx subjects, respectively. The rate of upgraded and downgraded GS (GS $>$ or $<3+4$ at RP) on SBx versus $\mathrm{TBx}$ was $38.5 \%$ vs. $16.8 \%$ and $1.6 \%$ and $2.0 \%$, respectively. The rate of upgrading was significantly associated with the presence of GP $4 \geq 11 \%$ versus $<11 \%$ (OR=4.2, 95\%CI $=1.2$ $19.0 ; p=0.024)$ and with the presence of cribriform pattern at biopsy specimens (OR6.7, 95\% CI $=2.4-24.7 ; p<0.001$ ). Discussion and Conclusion: Since the modification to the 
WHO-ISUP Gleason grading system was introduced, certain patterns such as cribriform glands that were originally scored as GP 3 have now been reclassified as GP 4. Consequently, the percentage of GS 7 cancers, in particular GS 3+4, significantly increased. Many authors demonstrated a significant correlation between the amount of GP 4 tissue in biopsy specimens and the pathologic parameters that were identified during RP. Specifically, a direct proportionality was observed with greater areas of GP 4 tissue in positive biopsy specimens and higher Gleason sum, pathologic stages, and total tumor volumes at RP. The present study demonstrated that TBx compared to SBx technique significantly reduced the risk of GS 3+4 upgrading at RP, and that may prevent some patients from avoiding lymphadenectomy during radical prostatectomy or from being monitored with active surveillance in case of patient's age $>70$ years. The rate of upgrading was significantly associated with GP4>10\%, mostly when cribriform pattern was present at biopsy specimen.

\section{2}

\section{OUTCOMES OF DIFFERENT PATTERN OF SEMINAL VESICLE INVASION IN PATIENTS TREATED WITH RADICAL PROSTATECTOMY}

Lorenzo Montesi $^{1}{ }$ Erika Palagonia ${ }^{1}$, Luca Leone ${ }^{1}$, Camilla Capretti $^{1}$, Alessia Cimadamore ${ }^{2}$, Lucio Dell' Atti ${ }^{1}$, Andrea Benedetto Galosi ${ }^{1}$ and Rodolfo Montironi ${ }^{2}$

${ }^{1}$ Clinic of Urology, AOU Polytechnic

of Marche Region, Torrette, Italy;
${ }^{2}$ Pathological Anatomy, AOU Polytechnic

of Marche Region, Torrette, Italy

Introduction: Ohori classification describes different patterns of invasion of seminal vesicle (SV) by prostate cancer: type 1 and 2 are continuous spread, respectively intraprostatic and extraprostatic, type 3 is a discontinuous metastatic invasion. Our objective is to evaluate the impact of Ohori patterns of invasion in a selected population of patients who underwent radical prostatectomy (RP). Patients and Methods: We evaluated 67 patients who underwent RP between 2007 and 2013 with a pT3bN0cM0 prostate cancer. The type of seminal vesicle invasion mechanism was evaluated by a dedicated uro-pathologist. We analyzed the biochemical recurrence $(B C R)$ in each kind of pattern of seminal vesicle invasion. We divided patients according adjuvant radiotherapy (ART) or neo-adjuvant hormonal therapy. We considered for each patient age, PSA at diagnosis, Gleason Score, global percentage of cancer, status of surgical margins, time of follow-up and incidence of BCR. Results: Mean follow-up was 104 (50-170) months. Mean age was 66.7 (55-77) years. In all the population 23 (33.8\%) patients experienced BCR, with a mean time of 26,7 months after radical prostatectomy. Ohori 1 was found in $4(6 \%)$ patients; no one experienced BCR. Ohori 2 was found in 50 (74.6\%) patients; 15 (30\%) experienced BCR. Ohori 3 was found in $13(19.4 \%)$ patients; $8(61.5 \%)$ experienced BCR. The bivariate analysis showed a statistically significant correlation ( $p=0.010)$ between the BCR and the type of SV invasion with an increased risk from type 1 to type 3
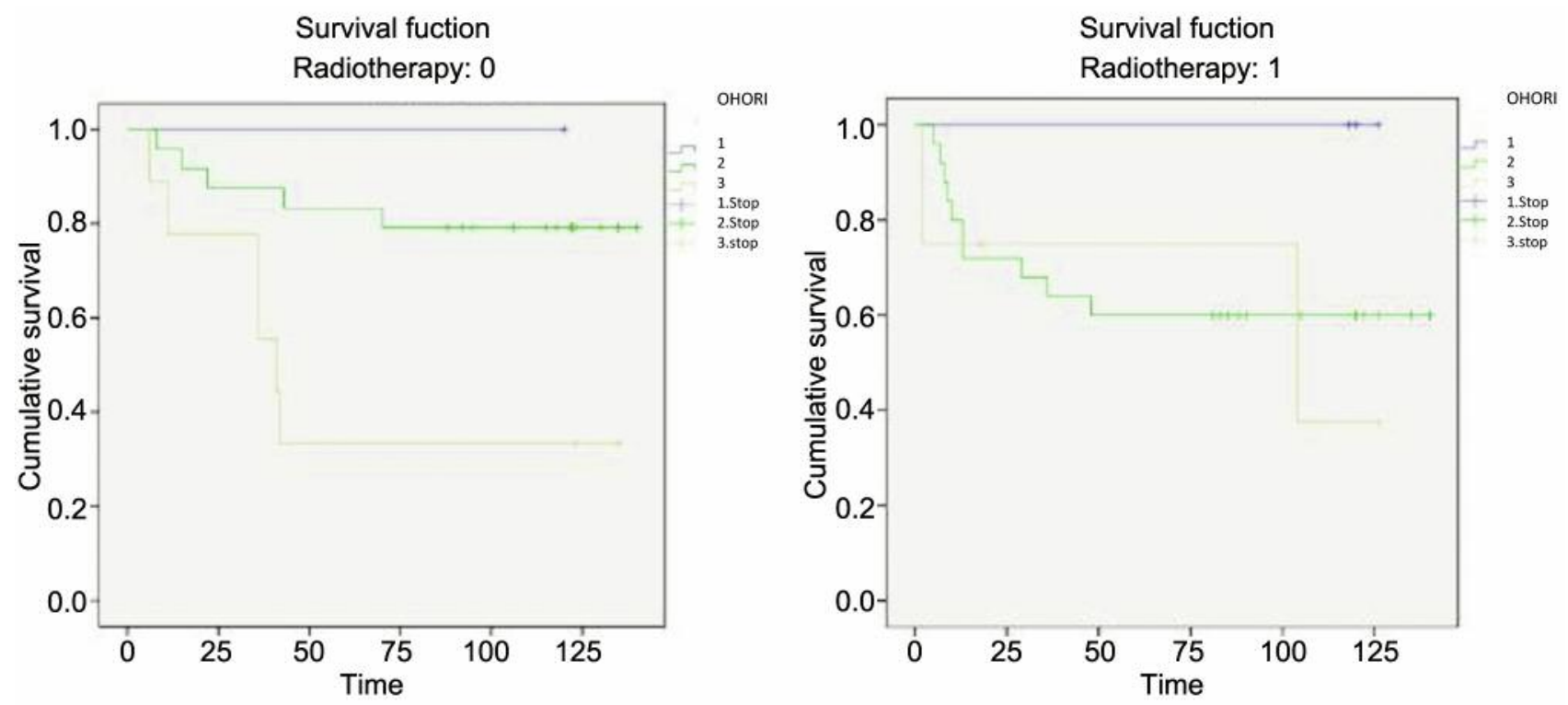

Figure 1. A: Survival curve in patients according to the type of Ohori. B: Survival curve in patients treated with radiotherapy according to the type of Ohori. 
$(\mathrm{OR}=31.6 \%)$. BCR occurred in 12 of $33(36 \%)$ patients who underwent adjuvant radiotherapy and in 11 of $34(32 \%)$ who did not undergo adjuvant radiotherapy. The linear regression showed that the risk of BCR is strongly correlated with Ohori type $3(\mathrm{OR}=49 \%, p=0.008)$ only in patients who did not underwent ART $(p=0.019)$ (Figure 1A). In patients receiving ART, the difference in terms of BCR was very close between type 2 and 3 with decreasing cases of BCR in patient with type 3 Ohori's (Figure 1B). BCR occurred in 8 of 17 patients with positive surgical margins (47\%) and in 15 of 50 patients without $(32 \%)$, without any correlation between BCR and each type of invasion. The statistical analysis did not show a correlation between Gleason Score and BCR. Furthermore, neoadjuvant therapy did not lead to changes in the type of Ohori and BCR, regardless of radiotherapy. Conclusion: Biochemical recurrence rate is directly correlated with the pattern of Ohori invasion in patients who underwent radical prostatectomy for pT3bN0cM0 prostate cancer. In type 1 Ohori invasion, adjuvant radiotherapy does not influence cancer-free survival. Radiotherapy do not change BCR of all Ohori II patients, while decreases the risk of BCR in patients with Ohori III. In patients with Ohori 3 invasion adjuvant radiotherapy is strongly indicated.

\section{3 \\ PRIMARY MYELOID SARCOMA OF THE PROSTATE: A DIAGNOSTIC CHALLENGE}

Giuseppe Maiolino $^{1}$, Federico Lanzi ${ }^{1}$, Filippo Gentile ${ }^{1}$, Gerardo Pizzirusso $^{1}$, Tommaso Chini ${ }^{1}$, Gabriele Gaziev ${ }^{1}$, Filippo Cecconi ${ }^{1}$, Gianni Vittori ${ }^{2}$ and Gabriele Barbanti ${ }^{1}$

${ }^{1}$ Department of Urology, Santa Maria

Alle Scotte Hospital, Siena, Italy;

${ }^{2}$ Department of Urology, Careggi Hospital, Carreggi, Italy

Introduction: Myeloid sarcoma (MS) is defined as the growth of myeloid blasts inside an extra-medullary site leading to the formation of one or more tumor masses that destroy the normal architecture pattern of the tissue. The most common sites are the skin, bone or lymph node, but it can affect every site of the human body. Generally, MS is divided in primary MS, when it is diagnosed in patient without a history of myeloid leukemia, MDS or myeloproliferative disorders but these develop with an involvement of blood or bone marrow after a period that ranges from five to twelve months and in secondary MS if it occurs with or following the onset of systemic bone marrow leukemia or represents a relapse of hematological disorder. It was included in acute myeloid leukemia (AML) and related neoplasms in the WHO Classification of Tumors of Hematopoietic and Lymphoid Tissue and it is maintained in this category also in the new 2016 revision of this classification. In literature, twenty-five cases of myeloid sarcoma of the prostate (MSP) have been described, among these, eleven are primary MS, twelve are secondary MS (two cases are not available to investigate). We describe another case of primary MSP with the development of an AML at high risk after three weeks. Case Report: A 66-year-old white male, with a history of cardiac surgery (mitral valve replacement and double aorta-coronary bypass) presented at Urology Department with lower urinary tract symptoms (LUTS) as nocturia and weak stream. The serological values of PSA revealed: 4.63 $\mathrm{ng} / \mathrm{ml}$ total PSA, $1.03 \mathrm{ng} / \mathrm{ml}$ free PSA and 22\% PSA ratio. At trans-rectal ultrasound (TRUS) the prostate appears with normal and symmetric shape, with volume of $44 \mathrm{~cm}^{3}$. Urologist's diagnosis was benign prostatic hyperplasia $(\mathrm{BPH})$ and he was treated by doxazosine and a prostate supplement. At that time the patient presented the following blood count: WBC $4.08\left(10^{3} / \mu \mathrm{l}\right)$, Hb 14.7 (g/dl), PLT $139\left(10^{3} / \mu \mathrm{l}\right)$. At follow-up after two months patient's serological total PSA values resulted increased to 7.31 $\mathrm{ng} / \mathrm{ml}$, with $1.04 \mathrm{ng} / \mathrm{ml}$ free PSA and 14\% PSA ratio. Suspecting it was acute prostatitis, it was prescribed an antibiotic therapy but the PSA exams performed after a week reveals gain to both total PSA $(12.38 \mathrm{ng} / \mathrm{ml})$ and free PSA (1.54 ng/ml). During this period, patient shows general symptoms such as weakness and nocturnal lowgrade fever and for this reason begins a cardiologic checkup that results normal. After these investigations, a prostate biopsy was performed. At time the prostate volume was $58 \mathrm{~cm}^{3}$ with normal echo-structure with small mixed area on the right side. In the same day, the patient turns to emergency room for uncontrolled gross hematuria that was managed with a three-way catheter bladder irrigation. In this setting, a blood complete count was performed, which showed: WBC $74.720\left(10^{3} / \mu \mathrm{l}\right), \mathrm{Hb} 14.7$ $(\mathrm{g} / \mathrm{dl})$, PLT $52\left(10^{3} / \mu \mathrm{l}\right)$. Hematologic consultation and a blood smear reveal an AML, more characterized in the following days when the patient is settled in Hematology Department. The immunophenotype profile resulted to be composed of two cell populations: $45 \%$ positive to CD34+ and 55\% CD34-, both positive to CD13, CD33, CD117, CD123, CD38 and MPO. The karyotype resulted complex and molecular investigation showed the presence of 9098 copies of WT-1/104 copies of ABL, the presence of NPM1 gene mutation ( 808 copies/104 copies of ABL, subtype A) and presence of FLT3 gene mutation in heterozygosity. These reports defined the hematological disorder as highrisk AML. The patient received chemotherapy based on FLAIE protocol (Fludarabine, Cytarabine, Idarubicin and Etoposide). During this period in Hematology Department, the bladder catheter was set in situ because the patient presented a urine stasis when he tried to remove the 
catheter. During hospitalization, the pathological report of previous prostate biopsy declared the absence of any epithelial neoplastic lesions, but it also underlined the presence of a diffuse infiltrate of cellular elements with blastic aspect (CD45+, CD68+/-, CD20-, CD3-, CD15-, MPO-) (Figure 1). After first chemotherapeutic cycle, the patient was discharged from the department in a full hematologic recovery state and after a week he was invited to clinic office to assess the therapeutic response. The bone marrow aspirate revealed a minimal residual disease. During the second chemotherapeutic cycle, the patient showed a total PSA of $5.66 \mathrm{ng} / \mathrm{ml}$. The catheter was removed and a suprapubic ultrasound was performed (the TRUS was contraindicated because of his hematologic state) that showed a reduced volume of prostate compared to the volume before chemotherapy. The MSP was confirmed not only by pathologic report, but by ultrasound (volume), serological (PSA) and hematology (blood count) data too (Figure 2). Discussion: An MSP is a diagnostic challenge with a high probability of initial misdiagnosis. There are many reasons: it is rare and incidence has been limited to case reports, the median age of presentation at diagnosis of MSP is 66.5 years (1) in which both BPH and prostate cancer (PC) are common, the clinical manifestations are unspecific such as progressive LUTS, acute urinary retention, hematuria and enlarged prostate at rectal examination. PSA has not shown a high specificity and sensibility: in our and other cases reported in literature PSA presented a rise in symptomatic phase and a reduction post-chemotherapy but in other cases it was at normal levels (2). Clinically, even unspecific, there are red flags who alert physicians on possible MSP, especially for secondary MSP: weakness, nocturnal low-grade fever, weight loss, hemorrhagic manifestation, increased susceptibility to infections or no response to BPH-therapy. A routine complete blood count is not recommended in every patient with LUTS or in patients who will undergo prostate biopsy but it will be useful in patient who manifest the red flag symptoms. For primary MS, complete blood count is useless, because in this case it is normal and the only possibility is a pathological diagnosis. Among primary MSP reported in literature the tissue diagnosis was obtained via TURP in $56.2 \%$, prostate biopsy in $31.2 \%$ and prostatectomy in $12.5 \%$ (1). Immunohistochemical markers useful in MS are MPO and CD68. In our case, the blast population in prostate was MPO negative. This is explained by the pathologist as waste antigen preservation due to fixation and coarctation of the tissue. Currently, imaging studies have demonstrated to observe a prostate lesion but without specific sign of MS. In the first line, ultrasound techniques (suprapubic and TRUS) can show an enlarged prostate while PET-CT and MRI of the pelvis can underline a suspected prostatic mass. Unfortunately,
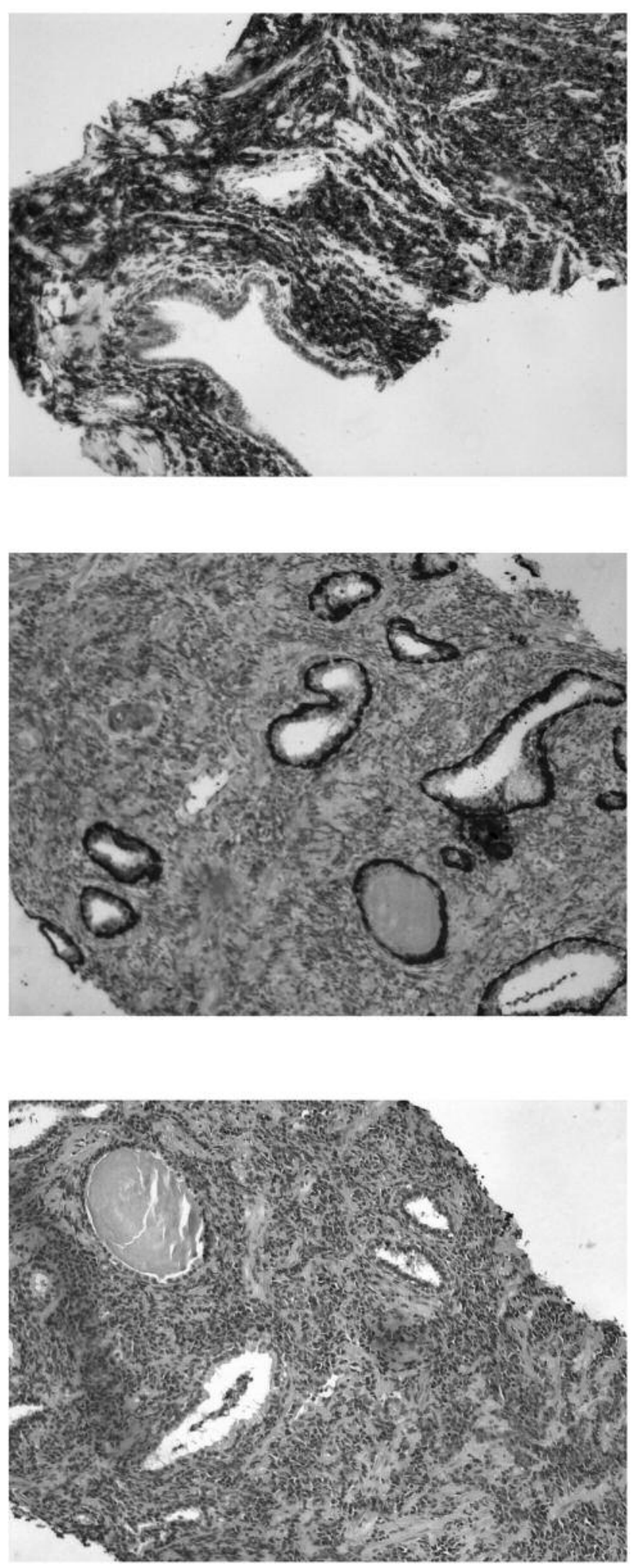

Figure 1. The first image (A) shows the expression of CD45 (CD45, $10 x)$. In the second image $(B)$ immunohistochemical antibody cocktail staining (p63/HMWCK/AMACR) was used, while the last one $(C)$ was stained with HE staining $(10 \times)$. 

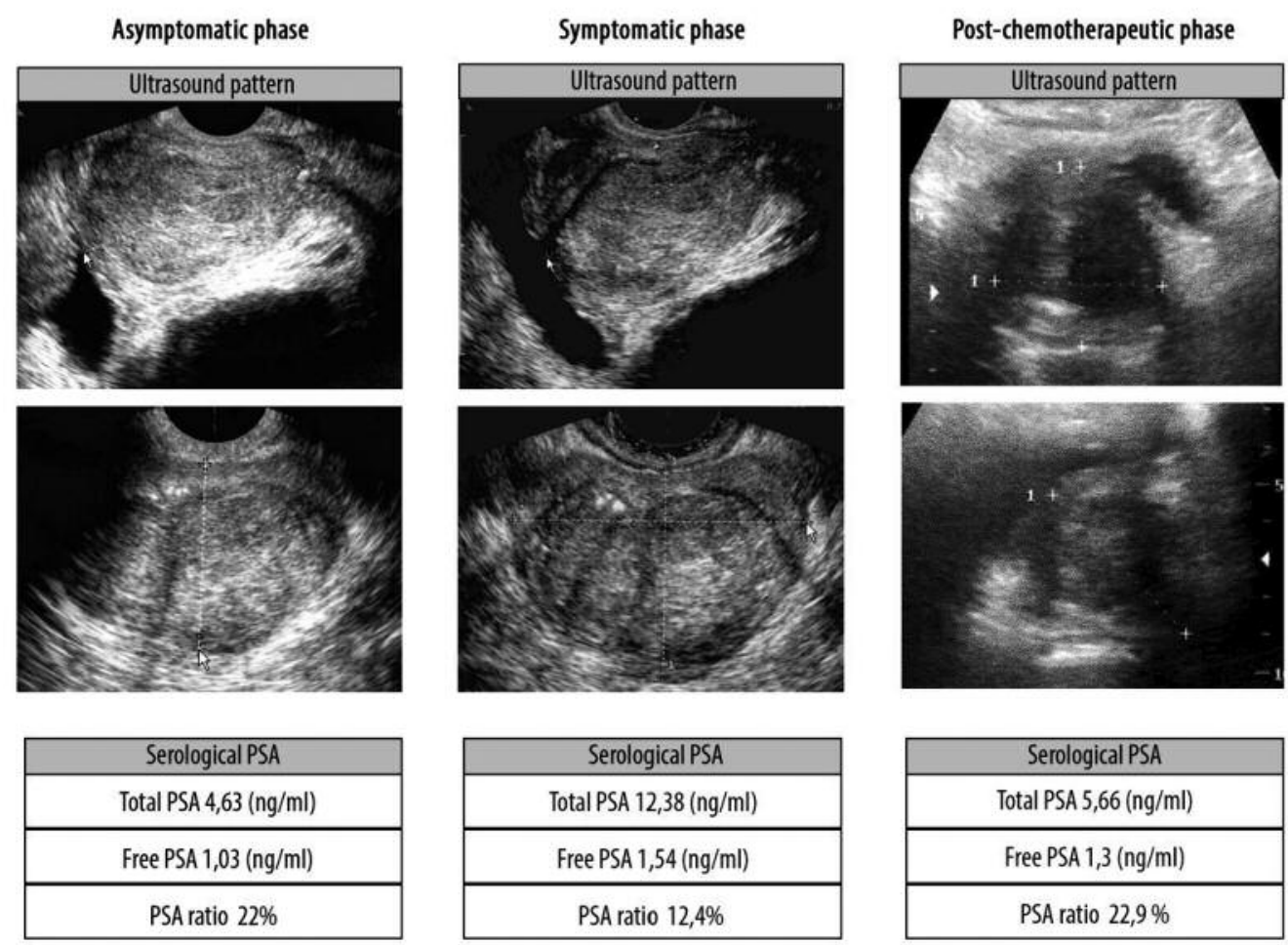

\begin{tabular}{|c|}
\hline Blood count \\
\hline WBC $4.08\left(10^{3} / \mu \mathrm{ll}\right)$ \\
\hline HB $14.7(\mathrm{~g} / \mathrm{dl})$ \\
\hline PLT $139\left(10^{3} / \mu \mathrm{ll}\right)$ \\
\hline
\end{tabular}

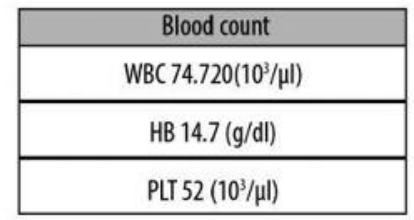

\begin{tabular}{|c|}
\hline Blood count \\
\hline WBC $0.63\left(10^{3} / \mu \mathrm{l}\right)$ \\
\hline HB $9.1(\mathrm{~g} / \mathrm{dl})$ \\
\hline PLT $21\left(10^{3} / \mu \mathrm{ll}\right)$ \\
\hline
\end{tabular}

Figure 2. The image represents in chronological order three groups of data of the case reported: ultrasound prostate pattern, serological level of PSA and main values of blood count. In the asymptomatic phase related to leukemia but with only LUTS symptoms the trans-rectal ultrasound shows an enlarged prostate, with normal PSA value and normal blood count value. In the symptomatic phase (weakness and nocturnal low-grade fever) of leukemia the ultrasound demonstrates an increased volume of prostate with normal echo-structure and a small mixed area on the right side, an elevated PSA and a pathological count of white blood cell. After chemotherapy the transabdominal ultrasound shows a reduction of volume prostate, of PSA and normalized blood count.

sensibility and specificity of imaging techniques have not been evaluated. Treatment for MS with or without systemic disease is typical therapy for AML (3). Conclusion: A clinical pattern of LUTS and enlarged prostate at rectal examination is a challenge for Urologists who have called to consider common and uncommon (or rare) diagnosis such as MSP. An accurate medical history and the use of blood complete count in high risk patients help physicians to suspect secondary MSP, while for primary MSP, the only tools are imaging study and tissue diagnosis. A multidisciplinary work-up among Urologists, Hematologists and Pathologists is necessary to avoid misdiagnosis.
1 Nguyen R and Sayar H: Primary myeloid sarcoma of the prostate: A case report and literature review. Case Repn Hematol 2018: 3604298, 2018. DOI: 10.1155/ 2018/3604298

2 Horvat N, Panizza PS, Costa FP and Viana PC: Prostate mass as a first manifestation of myeloid sarcoma in a patient with an occult acute myeloid leukemia-a case report. Urology 107: e5-e6, 2017. PMID: 28627396. DOI: 10.1016/j.urology.2017.05.043

3 Bakst RL, Tallman MS, Douer D and Yahalom J: How I treat extramedullary acute myeloid leukemia. Blood 118: 3785-3793, 2011. PMID: 21795742. DOI: 10.1182/blood2011-04-347229 
64

\section{STANDARD VS. EXTENDED PELVIC LYMPH NODE DISSECTION: PERI-OPERATIVE COMPLICATIONS AND SURVIVAL OUTCOMES FOLLOWING RADICAL CYSTECTOMY}

Matteo Soligo ${ }^{1}$, Valeria Lami ${ }^{1}$, Alessandro Morlacco ${ }^{1}$, Michele Colicchia ${ }^{1}$, Luca Boeri ${ }^{2}$, Vidit Sharma ${ }^{2}$, Igor Frank ${ }^{2}$, Stephen Boorjian² and R. Jeffrey Karnes ${ }^{2}$

${ }^{1}$ Urology Clinic, University of Padua, Padua, Italy;

${ }^{2}$ Department of Urology, Mayo Clinic,

Rochester, MN, U.S.A.

Introduction and Objectives: Radical cystectomy and lymph node dissection is the gold standard treatment for muscleinvasive bladder cancer and selected high-risk non-muscle invasive bladder cancer. However, the optimal extent of LND has not been established yet. Materials and Methods: Among 2200 patients who underwent radical cystectomy in a tertiary referral center between 1998-2017, we identified 1950 cN0M0 patients who underwent concurrent standard lymphadenectomy (SLND) or extended lymphadenectomy (ELND) for bladder cancer. Patients with incomplete demographic or clinical data were excluded. Lymphadenectomy templates were defined as follow: 1) SLND: internal iliac, presacral, obturator fossa and external iliac nodes; 2) ELND: as above plus common iliac nodes to aortic bifurcation and presacral nodes. Descriptive statistics were used to show baseline demographic and clinical data. Mann-Whitney and chi square test were used to compare SLND vs. ELND cohorts. Log-rank test and Kaplan-Meyer curves were used to compare survival outcomes of SLND and ELND. Multivariable Cox regression analysis was used to identify any predictors of cancer-specific survival (CSS) and overall survival (OS). Results: Choice of lymphadenectomy template was statistically different between 1998-2007 (SLND vs. ELND: 668, 65.4\% vs. 353, 35.6\%) and 2008-2017 (370, $38.8 \%$ vs. 559, 60.2\%). Overall, patients in the ELND cohort were younger (67 years, $p<0.0001)$ and had a lower Charlson Comorbidity index $(p=0.006)$. Pathologic tumor stage and non-urothelial histology distribution was similar among the cohorts (all $p>0.5$ ). Median follow-up was also similar (SLND vs. ELND, 41.1 vs. 40.1 months, $p=0.118)$. ELND was associated to a higher number of removed nodes (25 vs. 10, $p<0.0001)$ and positive nodes (3.8vs. 2.6, $p<0.0001)$ but to less positive surgical margins $(55,6 \%$ vs. 103, 9.9\%, $p=0.002)$. Incidence of intraoperative complications was higher in the ELND cohort $(p<0.0001)$; 30-day mortality and 30-day complications were similar $(p=0.45$ and $p=0.17)$. At multivariable analysis, pT3-T4 disease $(\mathrm{HR}=3.1, p<0.0001)$, pN1-3 stage $(\mathrm{HR}=2.36, p<0.0001)$, lymphovascular invasion (HR=1.45, $p=0.001)$, positive surgical margins $(\mathrm{HR}=1.65$, $p<0.0001)$ were associated with shorter CSS. Adjuvant

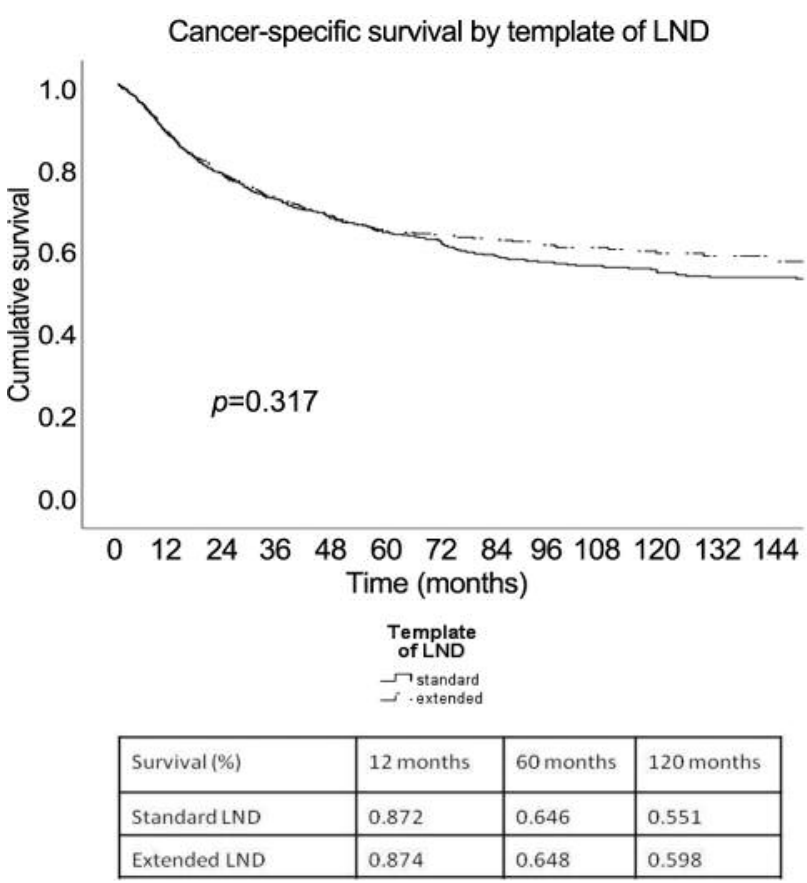

Figure 1. Kaplan-Meier curve showing cancer-specific survival by template of lymph node dissection (LND). Cumulative survivals at fixed time intervals are reported in the table on the right.

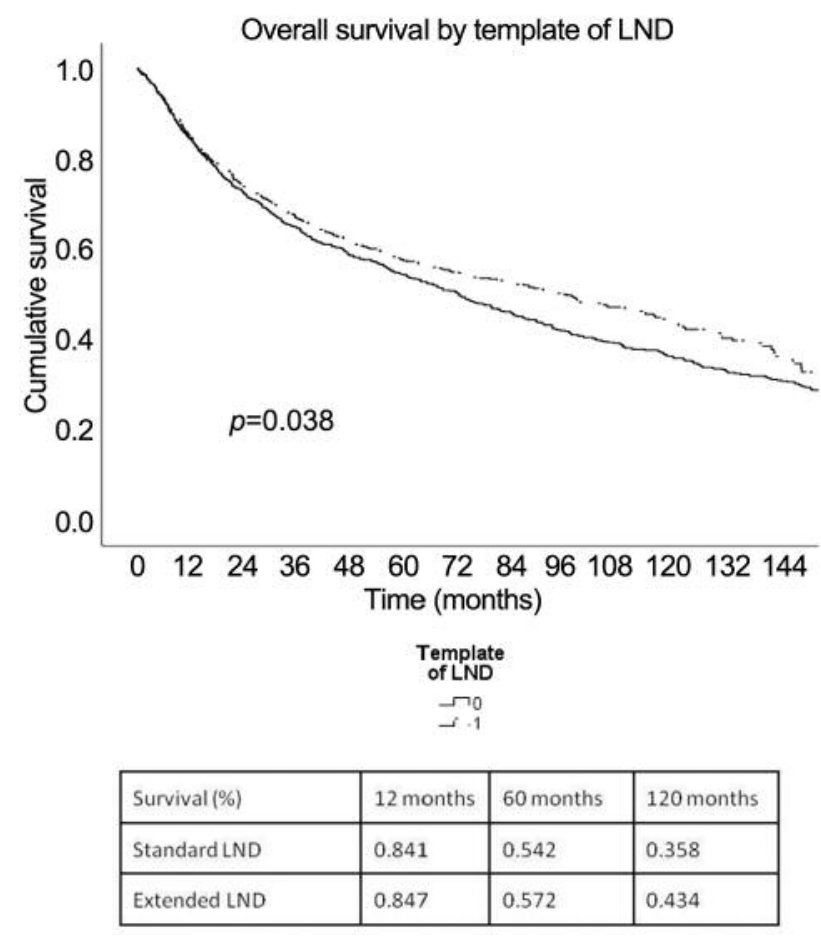

Figure 2. Kaplan-Meier curve showing overall survival by template of lymph node dissection (LND). Cumulative survivals at fixed time intervals are reported in the table on the right. 
chemotherapy $(\mathrm{HR}=0.56, p<0.0001)$ was associated with longer CSS. Kaplan-Meier curves did not demonstrate an association between CSS and the LND template $(p=0.317$ Figure 1); however, OS was significantly higher in the ELND cohort ( $p=0.038$ ) (Figure 2). Conclusion: ELND is a safe procedure in expert hands and may have a staging and therapeutic role in selected $\mathrm{cN} 0$ patients.

\section{5}

\section{MORBIDITY AND FUNCTIONAL OUTCOMES OF OPEN AND ROBOTIC SALVAGE RADICAL PROSTATECTOMY: DATA FROM A LARGE, RECENT, MULTICENTER SERIES}

Giancarlo Marra ${ }^{1}$, Paolo Gontero ${ }^{1}$, Paolo Alessio ${ }^{1}$, Giorgio Calleris ${ }^{1}$, Antonino Battaglia ${ }^{1}$, Claudia Filippin ${ }^{11}$, Stefania Munegato ${ }^{1}$, Fernando Munoz ${ }^{2}$, Marco Oderda ${ }^{1}$, Anna Palazzetti ${ }^{1}$, Francesca Pisano ${ }^{1}$, Umberto Ricardi ${ }^{3}$, Estefania Linares ${ }^{4}$, Rafael Sanchez-Salas ${ }^{4}$,

Ben Challacombe ${ }^{5}$, Paul Cathcart ${ }^{5}$, Prokar Dasgupta ${ }^{5}$, Sanchia Goonewardene ${ }^{5}$, Rick Popert ${ }^{5}$, Declan Cahill ${ }^{6}$, David Gillatt ${ }^{7}$, Raj Persad ${ }^{7}$, Juan Palou ${ }^{8}$, Steven Joniau ${ }^{9}$, Thierry Piechaud ${ }^{10}$, Salvatore Smelzo ${ }^{10}$, Alexandre De La Taille ${ }^{11}$, Morgan Roupret ${ }^{12}$, Simone Albisinni ${ }^{13}$, Roland Van Velthoven ${ }^{13}$, Alessandro Morlacco ${ }^{14}$, Sharma Vidit ${ }^{14}$, Giorgio Gandaglia ${ }^{15}$, Alexander Mottrie ${ }^{15}$, Joseph Smith ${ }^{16}$, Shreyas Joshi ${ }^{16}$, Gabriel Fiscus ${ }^{16}$, Andre Berger ${ }^{17}$, Monish Aron ${ }^{17}$, Andre Abreu ${ }^{17}$, Inderbir S. Gill ${ }^{17}$, Henk Van Der Poel ${ }^{18}$, Derya Tilki ${ }^{19}$, Nathan Lawrentschuk ${ }^{20}$, Declan G. Murphy ${ }^{20}$, Gordon Leung ${ }^{21}$, John Davis ${ }^{21}$ and Robert Jeffrey Karnes ${ }^{14}$

${ }^{1}$ Department of Surgery, Urology, University of Turin, AOU Città della Salute e della Scienza, Turin, Italy;

${ }^{2}$ Department of Radiotherapy, Parini Hospital, Aosta, Italy; ${ }^{3}$ Department of Radiotherapy, University of Turin, AOU

Città della salute e della Scienza, Turin, Italy;

${ }^{4}$ Department of Urology, Institut

Mutualiste Montsouris, Paris, France;

${ }^{5}$ Department of Urology, Urology Centre,

Guy's Hospital, London, U.K.;

${ }^{6}$ Department of Urology, Royal Marsden

Hospital, London, U.K.;

${ }^{7}$ Department of Urology, Bristol NHS

Foundation Trust, Bristol, U.K.;

${ }^{8}$ Department of Urology, Fundaciò

Puigvert, Barcelona, Spain;

${ }^{9}$ Department of Urology, Leuven

University Hospitals, Leuven, Belgium;

${ }^{10}$ Department of Urology, Clinique

Saint Augustin, Bordeaux, France;

${ }^{11}$ Department of Urology, CHU Mondor, Créteil, France;
${ }^{12}$ Department of Urology, Sorbonne Université, Hôpital Pitié-Salpêtrière, Paris, France;

${ }^{13}$ Department of Urology, Institut Jules Bordet, Université Libre de Bruxelles, Brussels, Belgium;

${ }^{14}$ Department of Urology, Mayo Clinic,

Rochester, MN, U.S.A.;

${ }^{15}$ Department of Urology, OLV Hospital, Aalst, Belgium;

${ }^{16}$ Department of Urology, Vanderbilt University,

Medical Center North, Nashville, TN, U.S.A.;

${ }^{17}$ Department of Urology, USC Norris Comprehensive

Cancer Center and Hospital, University

of Southern California, CA, U.S.A.;

${ }^{18}$ Department of Urology, Netherlands

Cancer Institute, Amsterdam, the Netherlands;

${ }^{19}$ Division of Urology, Martini-Klinik Prostate Cancer

Center, University Hospital Hamburg-Eppendorf,

Hamburg, Germany;

${ }^{20}$ Division of Urology, Division of Cancer Surgery, Peter

MacCallum Cancer Centre, Melbourne, VIC, Australia;

${ }^{21}$ Department of Urology, University of Texas MD

Anderson Cancer Center, Houston, TX, U.S.A.

Objectives: Historically, poor functional outcomes and high complication rates are linked to salvage radical prostatectomy (sRP). Nevertheless, promising results are attained in recent sRP series. We aimed to assess functional outcomes and complications of sRP, comparing the robotic and open techniques. Methods: From 2000 to 2016, we retrospectively collected data of SRP for recurrent prostate cancer (PCa) after local non-surgical treatment at 18 tertiary referral centers. Patients with: i) no functional outcomes or complications available; ii) follow up $<6 \mathrm{mo}$; were excluded from analysis. The Clavien-Dindo system was used to grade complications. We evaluated functional outcomes before sRP and at 6 and/or 1-year follow-up. Results: We included 395 sRP ( $n=186$ open; $n=209$ robotic). Comparing robotic and open cases, no significant baseline differences were present, apart from follow up $(p<0.001)$, pre-operative castration resistant $\mathrm{PCa}(p=0.0055)$ (higher for opens $\mathrm{RP})$ and sRP Gleason $(p=0.0159)$ (higher for robotic sRP). No extranodal metastasis was detected at pre-sRP imaging. Lower blood loss $(p<0.0001)$ and shorter hospital stay $(p<0.0001)$ were linked to robotic sRP, but no significant differences emerged in major $(10.1 \%, p=0.16)$ and overall complications $(34.9 \%$, $p=0.67$ ). Risk of rectal injuries and fistulas were $1.58 \%$ and $2.02 \%$, respectively; anastomotic stricture was more frequent in open-sRP $(16.6 \%$ vs. $7.7 \% ; p<0.01)$. Improved/unchanged continence occurred in $57.5 \%$, while severe ( 3 or more pads/day) incontinence was found in $24.6 \% ; 8.1 \%$ had preserved spontaneous or PDE-5 assisted erections (15.6\% potent before $\mathrm{sRP}$ had preserved erectile function compared to pre-sRP). Amongst nerve sparing procedures, three (11.5\%) in 26 men preserved spontaneous or PDE-5 assisted 
erections. On multivariable analysis, robotic-sRP was an independent predictor for continence preservation $(\mathrm{OR}=0.411, \quad 95 \% \mathrm{CI}=0.232-0.727, \quad p=0.022) ;$ previous hormonal treatment $(\mathrm{OR}=1.689,95 \% \mathrm{CI}=1.004-2.843$, $p=0.0484)$ and ASA score $(\mathrm{OR}=1.430,95 \% \mathrm{CI}=1.026-1.995$, $p=0.0349$ ) were associated to the occurrence of at least one complication. Conclusion: Nowadays, sRP shows a low risk of major complications and better functional outcomes than in past series. The robotic approach may reduce anastomotic strictures, blood loss, hospital stay, and improve continence.

\section{6}

\section{SURVIVAL OUTCOMES AND SALVAGE TREATMENTS FOR LOCAL BLADDER CANCER RECURRENCES FOLLOWING RADICAL CYSTECTOMY}

Matteo Soligo ${ }^{1}$, Giovanni Zecchini ${ }^{1}$, Michele Colicchia ${ }^{1}$, Alessandro Morlacco ${ }^{1}$, Luca Boeri ${ }^{2}$, Vidit Sharma ${ }^{2}$, Igor Frank ${ }^{2}$, Stephen Boorjian ${ }^{2}$ and

Robert Jeffrey Karnes ${ }^{2}$

${ }^{1}$ Urology Clinic, University of Padua, Padua, Italy;

${ }^{2}$ Department of Urology, Mayo Clinic,

Rochester, MN, U.S.A.

Introduction and Objectives: The 5-year bladder cancer recurrence rate after radical cystectomy ranges $15 \%-70 \%$; around $30 \%$ of these patients are diagnosed with local recurrence. Cisplatin-based chemotherapy is currently the standard treatment for local recurrences, whilst radiotherapy and surgery have marginal roles. However, salvage treatments are rarely curative. The aims of the present study are: to describe patterns of local recurrences; to identify the most effective salvage treatments and any predictors of cancerspecific survival (CSS) and overall survival (OS). Materials and Methods: Among $3700 \mathrm{cM} 0$ patients who underwent radical cystectomy in a tertiary referral center between 1980 2017, we identified 145 patients who experienced local recurrences during follow-up. Exclusion criteria were: incomplete demographic and clinical data; non-urothelial histology at RC; rare recurrence sites. Local recurrences were grouped as: recurrences in pouch/neobladder, bladder fossa/pelvis/vaginal cuff, regional nodes, $>2$ of the previous sites. Descriptive statistics were used to show baseline data. Multivariable Cox regression analysis was used to identify any predictors of CSS and OS. Results: 71 (49\%) patients had non-organ confined disease; 24 (16.6\%) had nodal involvement at radical cystectomy. Median time to recurrence was 10.7 (5.9-24.6) months; median follow-up time was 31 (15.9-72.9) months. All-cause death and cancer-specific death rates were $86.9 \%$ (126 pts) and $71 \%$ (103). The most common site of local recurrences was cystectomy bed (82,

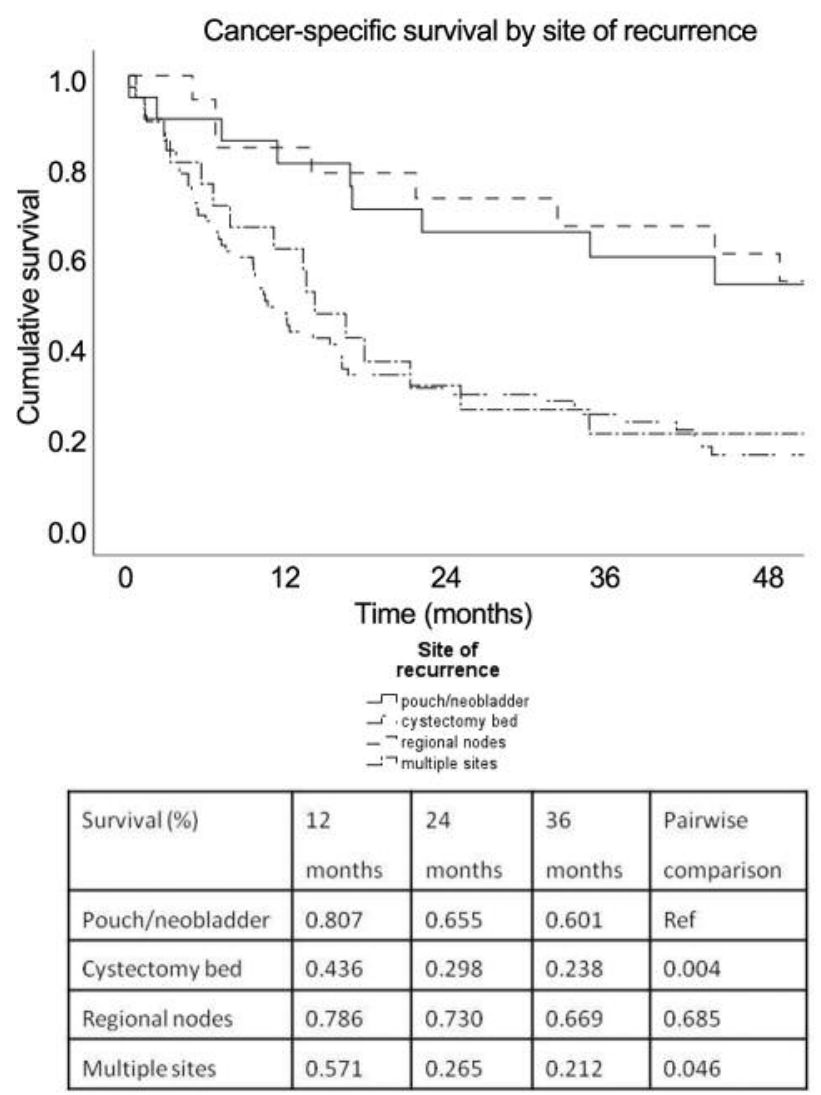

Figure 1. Kaplan-Meier curve showing cancer-specific survival by sites of recurrence. Cumulative survivals at fixed time intervals are reported in the table below the graphic.

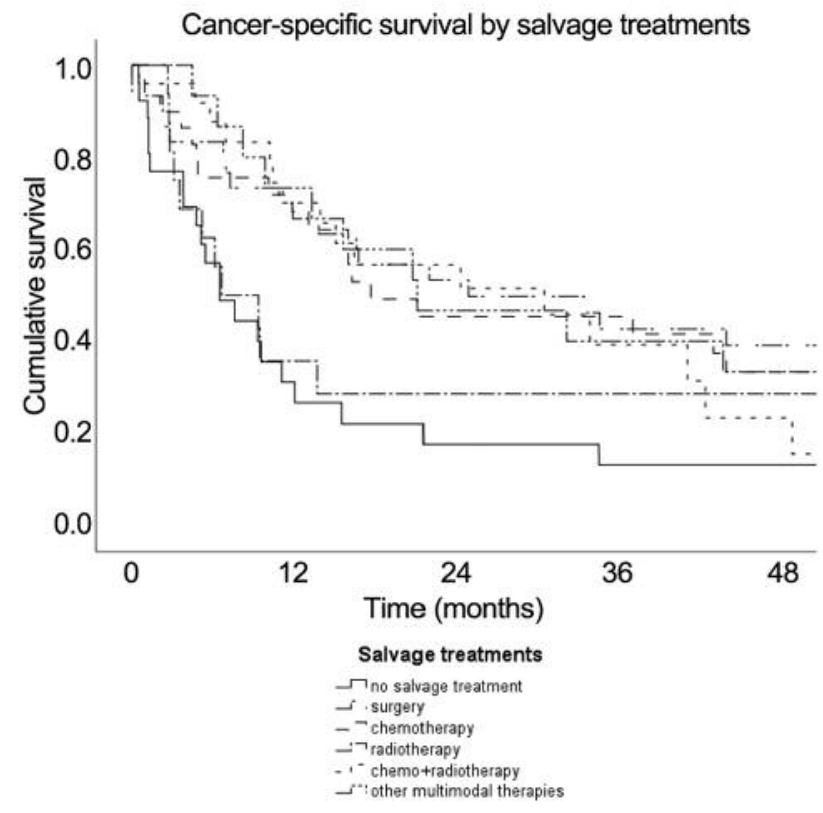

Figure 2. Kaplan-Meier curve showing cancer-specific survival by salvage treatments. Cumulative survivals at fixed time intervals are reported in the table below the graphic. 
$56.6 \%) .21 .4 \%$ of pts received surgery, $21.4 \%$ chemotherapy, $17.2 \%$ chemotherapy+radiation as salvage treatments. At Kaplan-Meier survival analysis, cystectomy bed recurrences $(p=0.004)$ and multiple sites of recurrence $(p=0.046)$ were associated to worse cancer-specific survival $(p=0.004$ and $p=0.046$, respectively, Figure 1). Besides, all treatments except for radiation were associated to a better cancer-specific survival as compared to no treatment (all $p<0.005$, Figure 2 ). At multivariable analysis, higher age at recurrence $(\mathrm{HR}=1.02$, $p=0.011)$, cystectomy bed recurrences $(\mathrm{HR}=3.2, p=0.003)$ and multiple recurrence sites $(\mathrm{HR}=2.4, p=0.03)$ were associated to shorter CSS. Chemotherapy (HR=0.45, $p=0.015)$, chemotherapy+radiation $(\mathrm{HR}=0.39, p=0.006)$ and miscellaneous multimodal treatments $(\mathrm{HR}=0.45, p=0.037)$ were significantly associated to longer CSS, without a significant difference among them. Higher age at recurrence $(\mathrm{HR}=1.03, p<0.0001)$ and cystectomy bed recurrences $(\mathrm{HR}=2.4, p=0.012)$ were associated to shorter OS. All treatments except for radiation were associated to longer OS (all $p<0.005$ ), but none demonstrated a significant better effect. Conclusion: Local recurrences are associated to poor prognosis, but outcomes differ significantly according to recurrence patterns. A multimodal approach including chemotherapy may achieve the best effect in prolonging CSS and OS.

\section{7}

\section{STEREOTACTIC BODY RADIATION THERAPY (SBRT) VS. MODERATE HYPOFRACTIONATED RADIOTHERAPY IN PROSTATE CANCER: PROPENSITY-SCORE BASED COMPARISON OF OUTCOME AND TOXICITY}

Ciro Franzese, Lucia Di Brina, Giuseppe D’agostino, Pierina Navarria, Fiorenza De Rose, Tiziana Comito, Davide Franceschini, Pietro Mancosu,

Stefano Tomatis and Marta Scorsetti

Department of Radiotherapy and Radiosurgery, Humanitas Clinical and Research Hospital, Rozzano, Italy

Introduction: Prostate cancer (PC) represents the second most common malignancy in the world and majority of patients have diagnosis of localized disease. Aim of the present study was to compare two cohorts of patients treated with moderate hypofractionation (MHRT) or stereotactic body radiation therapy (SBRT). Materials and Methods: We included patients treated between 2010 and 2015. Inclusion criteria were: adenocarcinoma of the prostate; class risks low or intermediate; WHO performance status 0-2. We evaluated rectal (RE), gastro-intestinal toxicity (GI) and genito-urinary (GU). Measures of outcome were biochemical disease-free survival (BDFS) and overall survival (OS). Propensity score
(PSM) was used to approximate the balance in covariates. Results: 209 patients were included, treated with MHRT $(n=109)$ or SBRT $(n=100)$. Median follow-up time was 37.4 months. Rates of BDFS at 1- and 3- years were $100 \%$ and $95 \%$, respectively. There was no significant difference between the 2 groups $(p=0.868)$. Rates of OS at 1 - and 3years were $100 \%$ and $97.1 \%$, respectively with no differences between the two groups ( $p=0.312)$. After PSM, no differences were observed in terms of acute and late RE and GI toxicity. While mild GU side effects were more common in SBRT group ( $45.5 \%$ vs. $19.5 \%)$, grade 2 and 3 toxicity were increased after MHRT $(11.7 \%$ vs. 2.6\%; $p=0.029)$. Conclusion: Moderate hypofractionation and SBRT are two effective and safe options for the treatment of low- and intermediate-risk prostate cancer. The analysis showed no difference in terms of disease's control and survival but increased moderate and severe toxicity after MHRT.

69

\section{SYSTEMATIC PROSTATE SAMPLING IN ADDITION TO MPMRI-TARGETED PROSTATE BIOPSY IMPROVES CANCER DETECTION RATE: RESULTS FROM A LARGE MULTICENTER RETROSPECTIVE SERIES}

$\underline{\text { Marco Oderda }}^{1}$, Giancarlo Marra $^{1}$, Giorgio Calleris ${ }^{1}$, Marco Falcone ${ }^{1}$, Simone Albisinni ${ }^{2}$, Emanuela Altobelli ${ }^{3}$, Eduard Baco ${ }^{4}$, Valerio Beatrici ${ }^{5}$, Marco Dellabella ${ }^{6}$, Jean-luc Descotes ${ }^{7}$, David Eldred-Evans ${ }^{8}$,

Giuseppe Fasolis ${ }^{9}$, Mariaconsiglia Ferriero ${ }^{10}$, Gaelle Fiard ${ }^{7}$, Alessandro Giacobbe ${ }^{11}$, Pardeep Kumar ${ }^{8}$, Vito Lacetera ${ }^{5}$, Pierre Mozer ${ }^{12}$, Giovanni Muto ${ }^{11}$, Rocco Papalia ${ }^{3}$, Alexandre Peltier ${ }^{13}$, Thierry Piechuad ${ }^{14}$, Tiziana Pietrangeli ${ }^{15}$, Giuseppe Simone ${ }^{10}$, Jean-Baptiste Roche ${ }^{14}$, Morgan Roupret ${ }^{12}$ and Paolo Gontero

${ }^{1}$ Department of Surgery, Urology, University of Turin, AOU Città della Salute e della Scienza, Turin, Italy; ${ }^{2}$ Department of Urology, Hopital Erasme, Université Libre de Bruxelles, Brussels, Belgium;

${ }^{3}$ Department of Urology, Campus Biomedico

University, Rome, Italy;

${ }^{4}$ Department of Urology, Oslo University

Hospital, Oslo, Norway;

${ }^{5}$ Department of Urology, Azienda Ospedaliera

Ospedali Riuniti Marche Nord, Pesaro-Fano, Italy;

${ }^{6}$ Department of Urology, INRCA-IRCCS

Ancona, Ancona, Italy;

${ }^{7}$ Department of Urology, Centre Hospitalier

Universitaire de Grenoble, Grenoble, France;

${ }^{8}$ Department of Urology, Royal Marsden

Hospital, London, U.K.; 
${ }^{9}$ Department of Urology, San Lazzaro Hospital, Alba, Italy; ${ }^{10}$ Department of Urology, Regina Elena

National Cancer Institute, Rome, Italy;

${ }^{11}$ Department of Urology, Humanitas

Gradenigo Hospital, Turin, Italy;

${ }^{12}$ Department of Urology, Pitié Salpétrière Hospital,

Assistance Publique - Hôpitaux de Paris,

Universités Paris Sorbonne, Paris, France;

${ }^{13}$ Department of Urology, Institut Jules Bordet,

Université Libre de Bruxelles, Brussels, Belgium;

${ }^{14}$ Department of Urology, Clinique

Saint Augustin, Bordeaux, France;

${ }^{15}$ Simple Departmental Unit Prostate Cancer,

INRCA-IRCCS Ancona, Ancona, Italy

Objectives: We evaluated the added value of performing a standard random sampling of the prostate in addition to mpMRI-targeted cores, considering prostate cancer (PCa) and clinically significant (cs) PCa detection, in a large multicenter series. Patients and Methods: Starting from a multicenter retrospective database of $2.115 \mathrm{mpMRI}-$ targeted biopsies, 1.119 patients were enrolled, from 2010 to 2017. The Koelis ${ }^{\mathrm{TM}}$ platform was used to perform the biopsies, which consisted in targeted (median 3 cores per target) and systematic (12 to 14 cores) sampling. Our primary outcomes consisted in overall and csPCa detection rate (CDR) of both target and systematic biopsies. Also, the potential predictors of PCa detection were investigated and tested. Results: About half (48\%) and one-third/33\%) of the patients undergoing biopsy were diagnosed respectively with PCa and csPCa, using targeted cores only. Detection for all cancers was augmented by $15 \%$ (and for csPCa by $12 \%)$, by considering the systematic sampling. Lesion scored as PI-RADS 3, 4 and 5 corresponded to 35\%, 69\%, and $92 \%$ of $\mathrm{PCa}$ detection rates, respectively. $\mathrm{PCa}$ diagnosis was consistently associated with higher PI-RADS score and positive digital rectal examination, whereas biopsy-naïve status was a predictor of csPCa. Conclusion: High CDR for both $\mathrm{PCa}$ and csPCa, in daily practice, is attained by targeted biopsies; however, the systematic sampling of the gland significantly improves detection rate. A significantly increased risk to be diagnosed with PCa was observed in patients with an elevated PI-RADS score and/or a positive DRE. Moreover, patients undergoing their first prostate biopsy showed a higher probability of csPCa detection.

\section{0}

ASSESSMENT OF THE VALUE OF AGE-ADJUSTED CHARLSON COMORBITY INDEX TO PREDICT PERIOPERATIVE AND SURVIVAL OUTCOMES OF RADICAL CYSTECTOMY
Tommaso Prayer Galetti $^{1}$, Matteo Soligo ${ }^{1}$, Alessandro Morlacco ${ }^{1}$, Valeria Lami ${ }^{1}$, Emanuele Serafin ${ }^{1}$, Fabio Zattoni ${ }^{2}$ and Filiberto Zattoni ${ }^{1}$

${ }^{1}$ Urology Clinic, University of Padua, Padua, Italy;

${ }^{2}$ Urology Clinic, Azienda Ospedaliera

di Gorizia, Gorizia, Italy

Introduction and Objectives: Despite improvements in surgical technique and post-operative care, open radical cystectomy (ORC) remains a procedure with high morbidity risk. Perioperative complications range 30-65\%, affecting survival, length of stay, readmission rate and total costs. The aim of this study was: to assess the incremental value of ageadjusted Charlson Comorbity index (ACCI) to predict perioperative complication and to identify any predictors of overall survival (OS). Materials and Methods: We queried an institutional prospective database to identify all cN0 patients which underwent ORC and pelvic lymphadenectomy since 12/2009. 413 patients with complete baseline, perioperative and follow-up data were considered for the final analysis. Collected data included baseline data (including ACCI), ORC pathologic data (histology, pathologic tumor stage, pathologic nodal stage, lymphovascular invasion, surgical margin status), perioperative complications and follow-up data. Descriptive statistics were used to show baseline and demographic data. Multivariable logistic regression analysis was used to test the association between ACCI and perioperative complication. Multivariable Cox regression analysis were used to identify any predictors of OS. Results: The median age was 70 (6376) years. The median BMI was $26.3(24.2-28.7) \mathrm{kg} / \mathrm{m}^{2}$. The median ACCI was 4 (2-3). 218 (52.8\%) patients had pT3-4 disease; 120 (29.1\%) had nodal involvement. $285(66.6 \%)$ patients received an ileal conduit diversion, 103 (24.9\%) an orthotopic ileal neobladder and 35 (8.5\%) an ureterocutaneous diversion. The median length of stay was 15 (13-20) days. The median follow-up was 18.2 (5.9-43.6) months. All-cause death and cancer-specific death rates were $39.5 \%$ (163 pts) and 29.3\% (29.3). The most common 30day complications were urine leakage $(61,14.8 \%)$, postoperative ileum $(47,11.4 \%)$ and wound complications $(44,10.7 \%)$. Sixty-three (15.3\%) patients had Clavien-Dindo $>3$ complications. 30- and 90-day readmissions were 23 $(5.6 \%)$ and $33(8 \%)$. At binary regression analysis, no significant association was found between potential predictors and recorded complications. At Cox regression analysis higher number of intraoperative blood transfusions, ( $\mathrm{HR}=1.15, p<0.0001)$, higher number of postoperative blood transfusions $(\mathrm{HR}=1.12, p=0.002)$, overall readmission within 90 days $(\mathrm{HR}=1.7, p=0.017)$, pathologic T3-4 stage $(\mathrm{HR}=2.96, p<0.0001)$, pathologic $\mathrm{N} 1-3$ stage $(\mathrm{HR}=2.33$, $p<0.0001)$ and higher ACCI $(\mathrm{HR}=1.12, p=0.024)$ were 
associated to shorter OS. In particular, ACCI $\geq 6$ was associated with the greatest increase in the hazard ratio $(\mathrm{HR}=1.64, p=0.017)$ at multivariable analysis. No significant protective factor for OS was found. Conclusion: ACCI is a reliable predictor of postoperative OS. Patients' selection for ORC should find the optimal balance between oncologic outcomes and the treatment burden. Further research is needed to distinguish between patients eligible for ORC and those eligible for alternative treatments.

\section{1}

\section{ORGAN SPARING SURGERY: IS THE CONSERVATIVE MANAGEMENT AN OPTION IN STAGE I TESTICULAR TUMORS?}

Emanuela Trenti ${ }^{1}$, Salvatore Mario Palermo ${ }^{1}$, Carolina D'elia ${ }^{1}$, Evi Comploj ${ }^{1}$, Dorjan Huqi ${ }^{1}$, Esther Hanspeter ${ }^{2}$, Susanne Baier ${ }^{3}$, Martin Maffei ${ }^{4}$, Justyna Waskjewicz ${ }^{4}$ and Armin Pycha ${ }^{1}$

${ }^{1}$ Department of Urology, Central

Hospital of Bolzano, Bolzano, Italy;

${ }^{2}$ Division of Pathology, Central

Hospital of Bolzano, Bolzano, Italy;

${ }^{3}$ Division of Oncology, Central

Hospital of Bolzano, Bolzano, Italy;

${ }^{4}$ Division of Radiotherapy, Central

Hospital of Bolzano, Bolzano, Italy

Introduction and Objective: Testicular cancer represents $1 \%$ of male neoplasms and 5\% of all urological tumors. Despite its relative low incidence and lack of data, the survival rate is very high, due to its high chemo-sensitivity and to the increasing number of early detection with ultrasound in the last years. Nevertheless, the current management is not changed and the guidelines suggest to perform an orchiectomy, if a malignant tumor is found on frozen sections, without distinction about the tumor-size. We present the oncological results of a series of 22 testicular tumors treated with testis sparing surgery (TSS) with the aim to assess the safety of this procedure in selected cases. Materials and Methods: Between 2005 and 2018, 21 TSS were performed at our department. Four patients were monorchid and 1 had a bilateral testicular cancer. The age ranged from 14 to 83 years (mean 38.4). Tumor markers (alfa-FP, beta-HCG, LDH) were assessed preoperative in all patients. All patients underwent inguinal access to the testis; frozen sections were made during the operation, associated, in case of TSS, with biopsies of the surrounding tissue. A staging computed tomography (CT) was performed after the surgery in all cases. Results: Tumor markers were negative in all patients except 3 , where they were only mildly elevated. Frozen sections showed a stromal tumor in 10 patients and a germ cell tumor (GCT) in the other 11 cases. All lesions were intraparenchymal, simply to resect and tumor size ranged from 7 to $40 \mathrm{~mm}$ (mean $14.2 \mathrm{~mm}$ ). The definitive histology confirmed the results of frozen sections in all cases. None of the 10 patients with stromal tumors showed histopathological risk factors and none showed relapse after a mean follow up of 48 months (range 18-108). Of the 11 cases of GCT, in 6 cases TSS was performed, due to solitary testis or synchronous bilateral tumor: in 5 of them a germ cell neoplasia in situ (GCNIS) was found in the definitive histology and 3 of these patients underwent radiotherapy (RT) while the other 2 underwent active surveillance. The other 5 patients underwent elective TSS; in 1 of these patients an orchiectomy was performed, for concomitant GCNIS in the definitive histology, because RT could damage the contralateral healthy testis. All these patients underwent regular follow up (range 3-120; mean 36 months); only 1 monorchid patient (9\%), under active surveillance for GCNIS, had relapse after 20 months and was treated with repeated TSS and RT. In 1 patient an orchiectomy for endocrine insufficiency was performed after 98 months, without finding a relapse. Only this patient and another one (18\%) needed hormonal replacement therapy. Conclusion: In selected cases, after adequate patient information, a TSS can be offered without compromising the oncological safety, even in case of a normal contralateral testis, to avoid a possible overtreatment and to attempt to preserve the endocrine functions and fertility. A delayed radical orchiectomy or RT could be offered to prevent recurrence in presence of GCNIS.

72

\section{OPEN RADICAL CYSTECTOMY IN ELDERS: COMPARISON OF PERIOPERATIVE AND SURVIVAL OUTCOMES IN A SINGLE CENTER SURGICAL SERIES}

Tommaso Prayer Galetti ${ }^{1}$, Matteo Soligo ${ }^{1}$, Alessandro Morlacco ${ }^{1}$, Valeria Lami ${ }^{1}$, Emanuele Serafin ${ }^{1}$, Fabio Zattoni ${ }^{2}$ and Filiberto Zattoni ${ }^{1}$

${ }^{1}$ Urology Clinic, University of Padua, Padua, Italy;

${ }^{2}$ Department of Urology, Azienda Ospedaliera

Di Gorizia, Gorizia, Italy

Introduction and Objectives: Open radical cystectomy (ORC) has been associated with high perioperative morbidity (3064\%) and mortality (2.3-8.0\%). Higher age at surgery has been reported as a risk factor for cancer-specific survival (CSS) and overall survival (OS). We aimed: 1) to compare perioperative and survival outcomes after ORC between patients $<75$ years and $>75$ years; 2 ) to identify any predictors of CSS and OS. Materials and Methods: We queried a prospectively maintained 


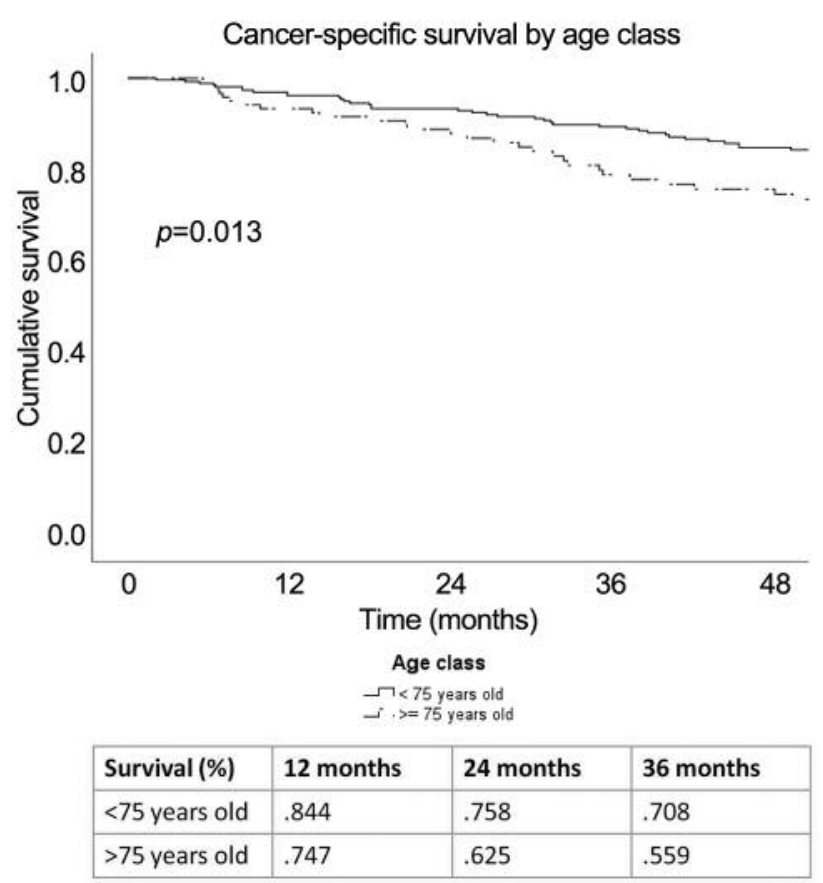

Figure 1. Kaplan-Meier curve comparing cancer-specific survival between patient $<75$ and $>75$ years old. Cumulative survivals at fixed time intervals are reported in the table below the graphic.

institutional database to retrieve all cNOM0 patients who underwent ORC at our center since 12/2009. 413 cNOM0 patients with complete baseline, pathologic, perioperative and follow-up data were identified and included in the final analysis. Patients were grouped by age class ( $<75$ or $>75$ years) at ORC. Descriptive statistics were used to test the association between age class and: baseline demographic and clinical data; ORC pathologic data; perioperative complications. KaplanMeyer curves were used to compare CSS and OS by age class. Multivariable Cox regression analysis were used to identify any predictors of CSS and OS. Results: A total of 285 (69\%) patients were $<75$ years, $128(31 \%)$ were $>75$ years. Patients $>75$ years had a lower BMI (25.24 vs. 26.8, $p=0.007)$, were more often active smokers (70.3\% vs. 24.2\%) and had a higher Charlson Comorbidity index ( 2 vs. $1, p<0.0001)$. Incidence of non-organ confined disease (pathologic T3-4 stage and/or pathologic N1-3 stage) was similar ( $<75 v s .>75: 58.2 \%$ vs. $60.2 \%, p=0.71$ ), but adjuvant chemotherapy was more often given to patients $<75$ years $(81,8.9 \% v s .13,10.2 \%)$. Patients $>75$ years had a slower median time-to-mobilization ( 5 vs. 4 days, $p<0.0001$ ) and to bowel recovery (4 vs. 3 days, $p=0.007$ ), but no differences in 30 -days postoperative complications $(41.4 \%$ vs. $41.8 \%, p=0.95)$ or Clavien $3-5$ complications $(16.4 \%$ vs. $14.7 \%, p=0.66)$. Kaplan-Meier curves showed an advantage in CSS $(p=0.013$, Figure 1$)$ and OS for patients $<75$ years $(p<0.0001$, Figure 2$)$. However, at

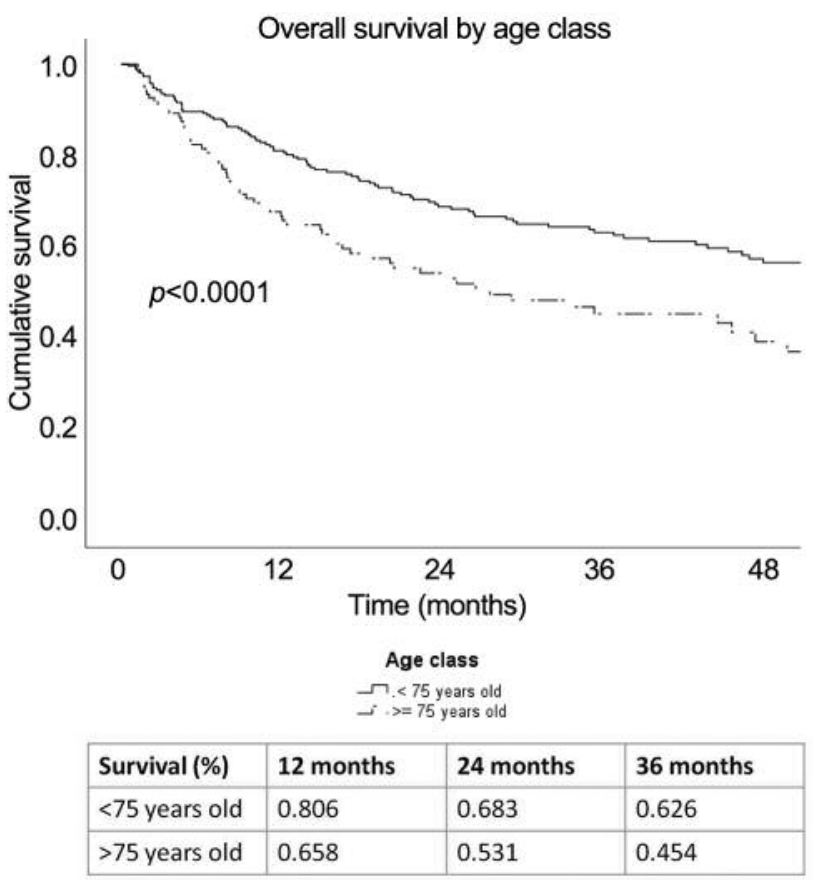

Figure 2. Kaplan-Meier curve comparing overall survival between patient $<75$ and $>75$ years old. Cumulative survivals at fixed time intervals are reported in the table below the graphic.

multivariable regression analysis only pathologic N1-3 stage (HR 2.1, $p=0.006$ ), positive surgical margins (HR=4.4, $p=0.008)$ and non-pelvic recurrences $(\mathrm{HR}=4.9, p<0.0001)$ were associated with shorter CSS. Adjuvant chemotherapy (HR $=0.58, p=0.04)$ was associated with longer CSS. Higher age-adjusted Charlson Comorbidity Index (ACCI: HR=1.09, $p=0.04)$, pathologic T3-4 stage $(\mathrm{HR}=2.2, p=0.003)$, pathologic N1-3 stage $(\mathrm{HR}=1.8, p=0.016)$, positive surgical margins (HR=4.2, $p=0.009)$ and non-pelvic recurrences $(\mathrm{HR}=2.6$, $p<0.0001)$ were associated with shorter OS. Comorbidities, as reported in ACCI, demonstrated a stronger association with OS than age $>75$, which was a risk factor for OS only at univariate analysis (HR=1.7, $p<0.0001)$. Conclusion: ORC may be considered the treatment of choice for fit elders with muscleinvasive bladder cancer or selected non-muscle invasive bladder cancer. ACCI is a better tool than crude age to weight the impact of aging on ORC outcomes.

\section{3}

\section{FUSION BIOPSY AND STANDARD BIOPSY IN NAIVE PATIENTS: PRELIMINARY RESULTS}

\section{Francesco Porpiglia $^{1}$, Enrico Checcucci ${ }^{1}$,} Matteo Manfredi ${ }^{1}$, Angela Pecoraro ${ }^{1}$, Diletta Garrou ${ }^{1}$, Giorgio Alleva ${ }^{1}$, Daniele Amparore ${ }^{1}$, Stefano De Luca ${ }^{1}$, 
Marco Cossu ${ }^{1}$, Massimiliano Poggio ${ }^{1}$, Francesca Ragni ${ }^{1}$, Ivano Morra ${ }^{1}$, Enrico Bollito ${ }^{2}$, Daniele Tota ${ }^{2}$ and Cristian Fiori ${ }^{1}$

${ }^{1}$ Department of Urology, AOU

San Luigi Gonzaga, Orbassano, Italy;

${ }^{2}$ Department of Pathology, AOU

San Luigi Gonzaga, Orbassano, Italy

Introduction and Objectives: Transrectal ultrasound-guided prostate biopsy has been the cornerstone of prostate cancer diagnosis. In men with suspected prostate cancer (PCa) TRUS-guided prostate biopsy (standard biopsy, SB) still remain the most used approach, even if it has a relatively poor diagnostic accuracy. Prostate cancer has traditionally been detected by randomly sampling the entire organ. For men undergoing initial biopsy with elevated PSA, prostate cancer detection rates are approximately $40-45 \%$ for the systematic 12-core SB. Subsequent serial biopsy results following previous negative biopsies may detect prostate cancer, even after many previous biopsies. However, the recent introduction of multiparametric magnetic resonance imaging (MP-MRI) now allows for imaging-based identification of prostate cancer. Multiparametric magnetic resonance imaging used as a triage test and subsequent MRI/TRUS fusion biopsy (FB) might allow to avoid unnecessary biopsy cores improving diagnostic accuracy. Our purpose is to evaluate the performance of standard biopsy and fusion biopsy in a cohort of biopsy naïve patients in terms of overall and clinically significant (CS) PCa diagnosis. Materials and Methods: In May 2014 the Biojet ${ }^{\circledR}$ (D\&K Technologies) software for FB was introduced in our Division. We analyzed retrospectively our prospectively maintained FB database and extracted the naïve patients who underwent SB+FB from July 2014 to August 2018. The inclusion criteria were: no previous prostate biopsy, age $\leq 75 \mathrm{yr}$, PSA level 4-15 $\mathrm{ng} / \mathrm{ml}$, negative DRE, examination with mpMRI. The minimum MRI requirements were multiparametric registration including T2w, DCE and DWI, a magnetic field strength of at least $1.5 \mathrm{~T}$ and a written report by the radiologist defining the accurate position of the suspected lesion in the prostate according to the the Prostate Imaging-Reporting and Data System (PI-RADS) scoring system version 2 classification. Three MRI-blinded urologists performed a 12-cores SB in all patients, then the Biojet software was activated and the same urologists performed the targeted biopsies of the PIRADS $>3$ index lesions ( 4 cores in case of $<8 \mathrm{~mm}$ lesion; 6 cores in case of $>8 \mathrm{~mm}$ lesion). Demographic, radiological and pathological variables were recorded. The overall detection rate (DR) and CS-PCa DR were evaluated. All the analyses were performed with $\mathrm{SAS}^{\circledR}$ Statistics Software. Results: A total of 225 patients were finally included. The overall DR of FB was $61.6 \%$, with a DR of CS-PCa of $52.4 \%$. The overall DR of SB was $41.3 \%$, with a DR of CS-PCa of $32.0 \% .50$ patients $(22.2 \%)$ had a positive FB with negative SB. The DR of CS PCa was 53.33\%. 13 patients $(5.7 \%$ ) had a negative FB with positive SB. The DR of CS PCa was $3.1 \%$. In this cohort of patients, SB was positive at the level of contralateral lobe with respect to MRI suspicious lesion in $9(69.2 \%)$ patients. In 83 (36.8\%) patients, both FB and SB were positive. In this cohort, the SB revealed a Gleason score upgrading in 2 (2.4\%) patients only. Discussion and Conclusion: There has been considerable concern regarding overdiagnosis and subsequent overtreatment of men with clinically indolent prostate cancer; thus, better characterization of prostate cancer is highly desirable. MRI/US-fusion-guided, targeted biopsies may potentially offer such an improvement. The integration of mpMRI into the diagnostic pathway for prostate cancer can add important information for further decision making. When highly suspicious MRI lesions were targeted, the great majority of cancers found were clinically significant, and few were insignificant. In our retrospective experience on biopsy naïve patients, the SB allowed to increase the overall $\mathrm{PCa}$ and the CS PCa DRs diagnosed by FB alone of $5.7 \%$ and $3.1 \%$ respectively. The limit of our study is that it is not a randomized study. A prospective randomized comparative study is mandatory in order to validate these preliminary results.

\section{5 \\ INTRODUCING INFORMATION ON GUT MICROBIOTA INTO TOXICITY MODELING: PRELIMINARY RESULTS FROM THE MICRO-LEARNER TRIAL}

Tiziana Rancati ${ }^{1}$, Barbara Avuzzi ${ }^{2}$, Loris De Cecco ${ }^{3}$, Nice Bedini' ${ }^{2}$, Sara Morlino' ${ }^{2}$, Sergio Villa ${ }^{2}$, Michela Dispinzieri ${ }^{2}$, Teresa Di Florio ${ }^{1}$, Fabio Badenchini ${ }^{1}$, Federica Palorini ${ }^{1}$, Alessandro Cicchetti ${ }^{1}$, Elisa Mancinelli ${ }^{3}$, Mara Serena Serafini ${ }^{3}$, Andrea De Vecchi ${ }^{3}$, Ester Orlandi ${ }^{4}$, Barbara Noris Chiorda ${ }^{2}$, Tommaso Giandini ${ }^{5}$ and Riccardo Valdagni ${ }^{6}$

${ }^{1}$ Prostate Cancer Program, Fondazione IRCCS Istituto Nazionale dei Tumori, Milan, Italy;

${ }^{2}$ Division of Radiation Oncology 1, Fondazione IRCCS Istituto Nazionale dei Tumori, Milan, Italy; ${ }^{3}$ Platform of Integrated Biology, Department of Applied Research and Technology Development, Fondazione IRCCS Istituto Nazionale dei Tumori, Milan, Italy; ${ }^{4}$ Division of Radiation Oncology 2 and Division of Radiation Oncology 1, Fondazione IRCCS Istituto Nazionale dei Tumori, Milan, Italy; ${ }^{5}$ Division of Medical Physics, Fondazione IRCCS Istituto Nazionale dei Tumori, Milan, Italy; 


\begin{tabular}{|c|c|c|}
\hline " p_Euryarchaeota;c_Methanococdi & - p_Euryarchaeota;c_Thermoplasmata & = Other;Other \\
\hline - $p_{j} c_{-}$ & $=p_{-} A C 1 ; C$ & $=p_{2}$ AC1;C_SHA-114 \\
\hline - P_AD $3 ; C \_A B S-6$ & - P_Acidobacteria;Other & $=p_{\text {_Acidobacteria; }}$ \\
\hline - P_Acidobacteria;c_Acidobacteria-5 & - p_Acidobacteria;c_Acidobacteria-6 & - P_Acidobacteria;c_Acidobacterila \\
\hline = p_Acidobacteria;c_BPC102 & - p_Acidobacteria;C_DA052 & = P_Acidobacteria;c_Holophagae \\
\hline - P_Acidobacteria;c_OS-K & = P_Acidobacteria;C_RB25 & - P_Acidobacteria;c_Solibacteres \\
\hline = P_Acidobacteria;c_Sva0725 & - p_Acidobacteria;c_TM1 & = p_Acidobacteria;c_[Chloracidobacteria] \\
\hline - P_Acidobacteria;c_ili1-8 & - p_Actinobacteria;Other & - p_Actinobacteria;c_ \\
\hline$=$ P_Actinobacteria;c_Acidimicrobilia & - p_Actinobacteria;c_Actinobacteria & = p_Actinobacteria;c_Coriobacter iia \\
\hline - P_Actinobacteria;c_MB-A2-108 & = p_Actinobacteriaj__Nitriliruptoria & = P_Actinobacteria;C_OPBA1 \\
\hline - P_Actinobacteria;c_Rubrobacteria & - P_Actinobacteria;c_Thermoleophilia & -p_Aquificae;c_Aquificae \\
\hline - P_Armatimonadetes; Other & - P_Armatimonadetes;c_0319-6E2 & - $p$ __Armatimonadetes;c_Armatimonadia \\
\hline I p_Armatimonadetes;c_Chthonomonadetes & - p_Armatimonadetes;c_SJA-176 & " p_Armatimonadetes;c_[Fimbrimonadia] \\
\hline = P_BRC1;C_NPL-UPA2 & $=$ P_BRC1;C_PRR-11 & - p_Bacteroidetes; Other \\
\hline - P_Bacter oidetes;c_ & - p_Bacteroidetes;C_BME43 & - p_Bacter oidetes;c_Bacter oidia \\
\hline - P_Bacteroidetes;c_Cytophagia & - p_Bacter oidetes; c_Flavobacterila & - P_Bacter oidetes;c_Sphingobacterila \\
\hline = p_Bacter oidetes;c_VC2_1_Bac22 & " p_Bacteroidetes;c_[Rhodothermi] & = p_Bacteroidetes;c_[Saprospirae] \\
\hline " p_Caldiserica;c_Caldisericia & p_Chlorobi;other & $=p_{\text {C_Chlorobix_BSV26 }}$ \\
\hline = p_Chlorobix_Chlorobia & - p_Chlorobix_Ignavibacteria & - p_Chlorobix_OPBS6 \\
\hline - chloroflexi, Other & - p_chloroflexi;c & - p_Chloroflexi;c_Anaerolineae \\
\hline - p_Chloroflexi;c_c0119 & - p_Chloroflexi;c_Chlor oflexi & - p_Chloroflexi;c_Dehalococcoidetes \\
\hline - p_Chloroflexi;c_Gitt-GS-136 & - p_Chloroflexi;c_Ktedonobacteria & =p_Chloroflexd;__MVP.21 \\
\hline = p_Chloroflexi;c_s085 & - p_Chloroflexi;c_SAR202 & $=$ p_Chloroflex;;__SHA-26 \\
\hline - P_Chloroflexi;c_TK10 & " P_Chloroflexi;c_TK17 & - p_Chloroflexi;c_Thermomicrobia \\
\hline - p_Cyanobacteria;Other & - P_Cyanobacteria;c_4cod-2 & = p_Cyanobacteria;c_Chlor \\
\hline - p_Cyanobacteria;c_ML635J-21 & - p_Cyanobacteria;c_Nostocoph & - P_Cyanobacteria;c_Oscillatoriophycideae \\
\hline " p_cyanobacteria;c_Synechococcophycideae & - p_Deferribacteres;c__Deferrib & $=$ p_Elusimicrobia;c_4-29 \\
\hline o_Elusimicrobia;c_Elusimicrobia & "p_Elusimicrobia;c_Eñdomicrobia & $=P_{-} F B P, C_{-}$ \\
\hline - P_Fibrobacteres;c_Fibrobacteria & - P_Fibrobacteres;C_TG3 & - p_Firmicutes;Other \\
\hline - p_Firmicutes;c_Bacilli & - p_Firmicutes;c_Clostridia & - p_Firmicutes;c_Erysipelotrichi \\
\hline = p_Fusobacteria;c_Fusobacterila & P_GNO2; Other & $=p_{-}$GN02;C_B834 \\
\hline$=p_{-}$GNO2;C_BD1-5 & $=$P_GNO2;C_GKS2-174 & $=P_{-}$GNO4;C_MSB-5AS \\
\hline - p_Gemmatimonadetes, Other & - P_Gemmatimonadetes,c_Gemm-1 & -p_Gemmatimonadetes;c_Gemm-2 \\
\hline - p_Gemmatimonadetes;c_Gemm-3 & - p_Gemmatimonadetes;c_Gemm-4 & - p_Gemmatimonadetes;c_Gemm-5 \\
\hline = P_Gemmatimonadetes,c_Gemmatin & " P_Gemmatimonadetes,c_J-ETNP-Z39 & $=p_{-} K S B 3 ; c_{-}$ \\
\hline "p_Lentisphaerae;c_[Lentisphaeria] & = P_NKB19; Other & $=p_{-}$NKB19; \\
\hline = p_Nitrospirae,c_Nitrospira & - P_OD1;c & $=p_{-}$OD $1 ; c_{-} A B Y_{1}$ \\
\hline - P_OD1;C_ZB2 & "p_OP11;C_WCHB1-64 & - p_OP3; Other \\
\hline - P_OP3;C_BD4-9 & - p_OP3;c_PBS-25 & = p_OP3;c_koll11 \\
\hline - P_OP8;C_OPB_1 & - P_OP8;C_OP8_2 & - P_OP8;C_SAW1_B6 \\
\hline$=P_{2} O P 9 ; C_{-} J S 1$ & $=P_{-} O P 9 ; c_{-} O P B \overline{4} 6$ & $=p_{-}$PAUC34f;c_ \\
\hline
\end{tabular}
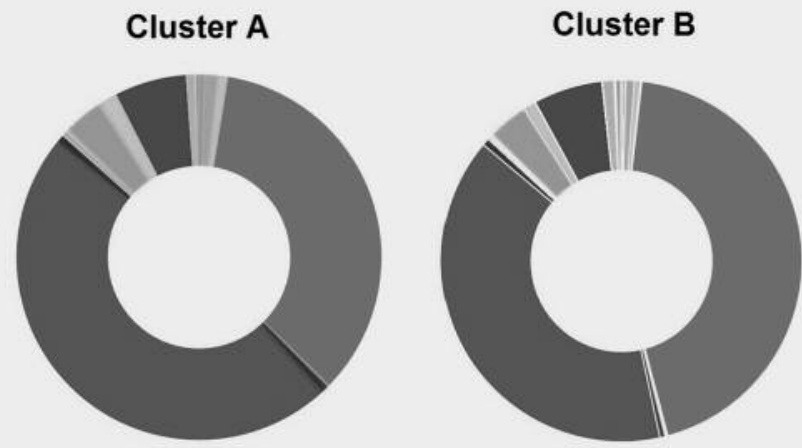

The following bacterial classes are present with different abundance in microbiota cluster $A$ and $B$ with $p$-value $<0.01$

$\begin{array}{ll}\text { Firmicutes_Other } & \rightarrow \text { more expressed in Cluster A } \\ \text { Firmicutes_Clostridia } & \rightarrow \text { more expressed in Cluster A } \\ \text { Chlorobi_BSV26 } & \rightarrow \text { more expressed in Cluster A } \\ \text { Verrucomicrobia_Opitutae } & \rightarrow \text { more expressed in Cluster A }\end{array}$

Nitrospirae_Nitrospira
Proteobacteria_Other
Gemmatimonadetes_Gemm-5
Bacteroidetes_Bacteroidia

$\rightarrow$ more expressed in Cluster A $\rightarrow$ more expressed in Cluster $A$

$\rightarrow$ more expressed in Cluster $A$

$\rightarrow$ more expressed in Cluster $B$

Figure 1. Average Operational Taxonomic Unit composition at Class level for patients in microbiota clusters A and B.

${ }^{6}$ Department of Hemato-oncology and Oncology, Prostate Cancer Program, Division of Radiation Oncology 1, University of Milan and Fondazione IRCCS Istituto Nazionale dei Tumori, Milan, Italy

Background/Aim: A mono-institutional trial (MICRObiota, infLammatory Environment, clinicAl and Radiomic features as predictors of Normal tissue response in radiotherapy for prostatE and head-and-neck canceR - MICROLEARNER; ClinicalTrials.gov NCT03294122), was set up in 2017 to investigate the role of gut/saliva microbiota in driving radioinduced toxicity after radaiotherapy for prostate (PCa) and head\&neck cancers. The main idea behind MICROLEARNER is to provide new insight about the response of 


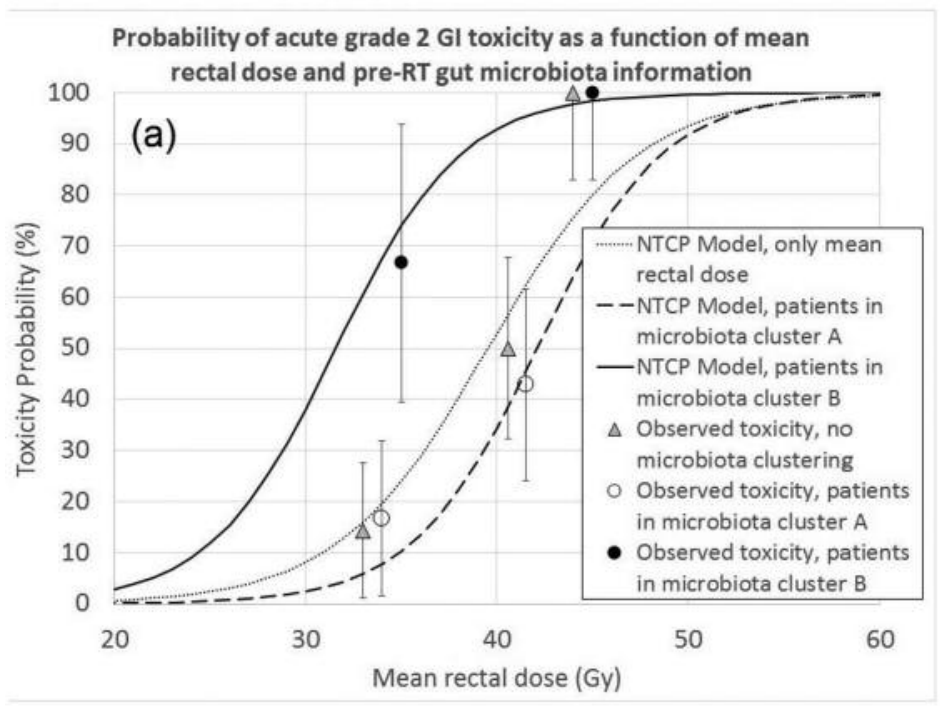

(b)

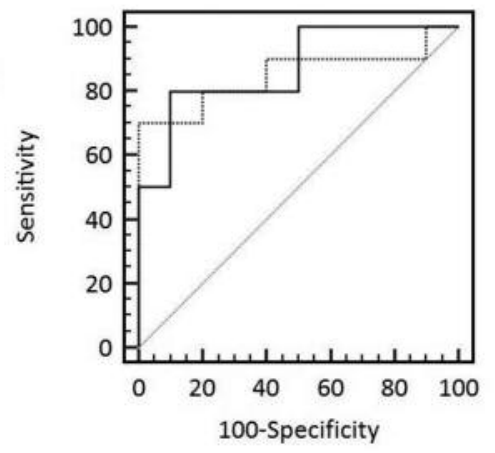

$\ldots$ NTCP model $(1)$ :
only mean rectal dose $($ AUC $=0.85)$
- NTCP model $(2):$
mean rectal dose + microbiota clustering (AUC=0.87)

Figure 2. (a) Probability fo acute grade 2 gastro-intestinal toxicity as a function of mean rectal dose and gut microbiota clustering. Observed complication rates are also reported (symbols with error bars representing the 68\% confidence interval in observed frequencies as calculated from proportions in the study population and based on normal distribution of event). (b) ROC curves for NTCP models with and without inclusion of gut microbiota clustering as dose-modifying factor.

healthy tissues to radiotherapy by using information from the micro-environment, obtained by biological measurements and imaging. Particularly, the role of gut and saliva microbiota in driving radio-induced toxicity is investigated. Recent research indicates that microorganisms living in the host have a role in initially activating or controlling inflammation from external causes. A dysbiotic milieu may change the metabolism of the host and provoke increased inflammation (1). Interesting results came from preclinical studies: germfree mice are resistant to lethal radiation enteritis, indicating that the microbiome controls radiation-induced intestinal disease processes (2). In a mouse model, the most significant shift in microbial composition was detected 6 weeks after irradiation and specifically noted in the colon, with reduced Firmicutes and increased abundance of Proteobacteria and of six genera, including Akkermansia spp and Bacteroides. It was also demonstrated that oxidative stress induced expansion of Proteobacteria, while the same oxidative stress was detrimental on Firmicutes, which are oxygen-sensitive bacteria $(3,4)$. MICRO-LEARNER aims at translating preclinical findings into an observational clinical trial setting, preliminary data for $\mathrm{PCa}$ are here presented, with particular focus on introduction of information on gut microbiota into a normal tissue complication probability model (NTCP) for acute gastro-intestinal toxicity in the PCa cohort. Patients and Methods: A total of 130 consecutive PCa patients receiving radiotherapy at the National Cancer Institute (Milan) were enrolled in the discovery population of the MICROLEARNER trial, 70 patients were enrolled in the validation population. All PCa patients received conventional (78Gy @2Gy/fr) or moderately hypofractionated (65Gy @2.6Gy/fr) VMAT+IGRT in $5 \mathrm{fr} /$ week. A detailed evaluation was done pre-treatment, during radiotherapy and at the end of the treatment, including a prospective collection of details on oncological treatment, comorbidities, drugs, habits and organ functioning (both clinician- and patient-reported). Gut microbiota measurement was performed before radiotherapy (baseline) and at the end of treatment. Stool samples were collected using gut-OMNIgene devices (Oragene). DNA extraction was carried out using the QIAamp-DNA-StoolMini-Kit (Qiagen). The bacterial 16S ribosomal-RNA reads were analyzed with the QIIME software and pooled in Operational Taxonomic Units (OTUs) with Uclust software. Grade 2 (G2) CTCAE acute intestinal toxicity was the primary endpoint of the here presented preliminary analysis. For this initial evaluation 20 patients were selected: 10 with G0 and 10 with G2 acute intestinal toxicity. All patients were without any intestinal symptom at baseline (G0 before radiotherapy). Unsupervised clustering using fuzzy c-means algorithm was used to separate the patients into 2 microbiota clusters, based on relative abundance of OTUs at bacterial class level in microbiota before radiotherapy start (baseline microbiota). Information on microbiota clustering was introduced as a dose-modifying factor into a logit NTCP model (characterized by D50=dose associated to $50 \%$ toxicity probability and steepness parameter $\mathrm{k}$ ). Mean dose to the rectum was chosen as dosimetric predictor [as already found in the literature (5)]. Results: Unsupervised clustering 
identified 13 patients included in a first microbiota cluster (A) and 7 in a second cluster (B), average OTU composition for patients in clusters A and B are presented in Figure 1. Figure 1 also reports on bacterial classes which were present with significantly different ( $p$-value $<0.01$ ) abundance in clusters A and B. 4/13 (31\%) and 6/7 (86\%) patients with toxicity were found in clusters A and B, respectively $(p=0.019)$. Microbiota clustering resulted in $\mathrm{AUC}=0.75$ (95\% $\mathrm{CI}=0.51-0.91)$ for toxicity discrimination. An NTCP model including only mean rectal dose had D50=49Gy, $\mathrm{k}=16(\mathrm{AUC}=0.85,95 \% \mathrm{CI}=0.62$ $0.97)$. When clustering was introduced, the fitted parameters were $\mathrm{k}=20.5$, D50=42Gy for cluster A vs. D50=32Gy for cluster B: with microbiota clustering resulting in a dosemodifying factor of 0.76 (B vs. A) $(\mathrm{AUC}=0.87,95 \% \mathrm{CI}=0.65$ $0.98)$. Introduction of information on microbiota clustering into NTCP modelling also resulted in significant improvement in goodness of fit and calibration. Model curves are reported in Figure 2. Conclusion: This preliminary analysis demonstrates the possibility of introducing patientspecific microbiota information into NTCP model, through use of unsupervised clustering to exploit the whole microbiota information (176 classes) without dramatically increasing the number of features to be included in the model. Results obtained in a small sample of PCa patients seem promising in indicating that patients with/without radioinduced acute intestinal toxicity have different constitutional gut microbiota profiles. Introduction of microbiota clustering into NTCP highly improves model performance. If confirmed in the whole accrued population, this could represent an important finding, not only for prediction of tox but also for design of possible interventional trials to reduce toxicity by modification of gut microbiota before radiotherapy start, by use of diet, probiotics and prebiotics.

1 Ferreira MR, Muls A, Dearnaley DP and Andreyev HJ: Microbiota and radiation-induced bowel toxicity: lessons from inflammatory bowel disease for the radiation oncologist. Lancet Oncol 15(3): e139-147, 2014. PMID: 24599929. DOI: 10.1016/S1470-2045(13)70504-7

2 Crawford PA and Gordon JI: Microbial regulation of intestinal radiosensitivity. Proc Natl Acad Sci USA 102(37): 13254-13259, 2005. PMID: 16129828. DOI: $10.1073 /$ pnas.0504830102

3 Morgan XC, Tickle TL, Sokol H, Gevers D, Devaney KL, Ward DV, Reyes JA, Shah SA, LeLeiko N, Snapper SB, Bousvaros A, Korzenik J, Sands BE, Xavier RJ and Huttenhower C: Dysfunction of the intestinal microbiome in inflammatory bowel disease and treatment. Genome Biol 13(9): R79, 2012. PMID: 23013615. DOI: 10.1186/ gb-2012-13-9-r79

4 Winter SE, Winter MG, Xavier MN, Thiennimitr P, Poon V, Keestra AM, Laughlin RC, Gomez G, Wu J, Lawhon SD, Popova IE, Parikh SJ, Adams LG, Tsolis RM, Stewart
VJ and Bäumler AJ: Host-derived nitrate boosts growth of E. coli in the inflamed gut. Science 339(6120): 708-711, 2013. PMID: 23393266. DOI: 10.1126/science.1232467

5 Landoni V, Fiorino C, Cozzarini C, Sanguineti G, Valdagni $\mathrm{R}$ and Rancati T: Predicting toxicity in radiotherapy for prostate cancer. Phys Med 32(3): 521-532, 2016. PMID: 27068274. DOI: 10.1016/j.ejmp.2016.03.003

\section{6 \\ PATIENT REPORTED OUTCOMES IN PROSTATE CANCER, A MOBILE-HEALTH ESSAY (PROPHECY)}

Bruno Fionda $^{1}$, Andrea D'Aviero ${ }^{2}$, Anna Rita Alitto ${ }^{1}$, Vincenzo Frascino ${ }^{1}$, Antonio Piras ${ }^{2}$, Francesco Catucci ${ }^{1}$, Giovanni Palazzoni ${ }^{2}$, Giuditta Chiloiro ${ }^{1}$, Loredana Dinapoli ${ }^{1}$, Vito Lanzotti ${ }^{1}$, Vincenzo Valentini ${ }^{2}$ and Giovanna Mantini ${ }^{2}$

${ }^{1}$ Area Radioterapia Oncologica, Dipartimento di Diagnostica per Immagini, Radioterapia Oncologica ed Ematologia, Fondazione Policlinico Universitario "A. Gemelli" IRCCS, Rome, Italy;

${ }^{2}$ Istituto di Radiologia, Università Cattolica del Sacro Cuore, Dipartimento di Diagnostica per Immagini, Radioterapia Oncologica ed Ematologia, Fondazione Policlinico Universitario "A. Gemelli" IRCCS, Rome, Italy

Aim: The scope of this study was to develop an electronic PRO tool to systematically assess and, possibly, to reduce, the burden of radiation therapy side events on prostate cancer patients' quality of life both during the treatment and the follow-up. Materials and Methods: To materialize such an electronic PRO tool, we proceeded with a four-step process. In the first step a comprehensive literature search was conducted to review the existing items from dedicated QoL questionnaires already validated in literature. The second part was to generate a set of adequate questions and answers for the patients with the help of a dedicated psycho-oncologist. The further step was to identify a reliable scale to report adverse events which was linked to the number of possible responses to each item included. The last phase was to implement a user-friendly interface with the help of graphic experts for patients including all the items previously organized as shown in Figure 1. Results: Our results need to be evaluated in the light of a wider project called VALEO+ ${ }^{\circledR}$ thought to provide patients and their caregivers with multiple applications to help them copy the entire radiation therapy process. We performed a literature research to collect the existing items from dedicated QoL questionnaires already validated and we displayed a complete list of all the most relevant items. Subsequently, a list of questions and answers, that recalled the CTCAE v4.03 scale of toxicity was elaborated. We 


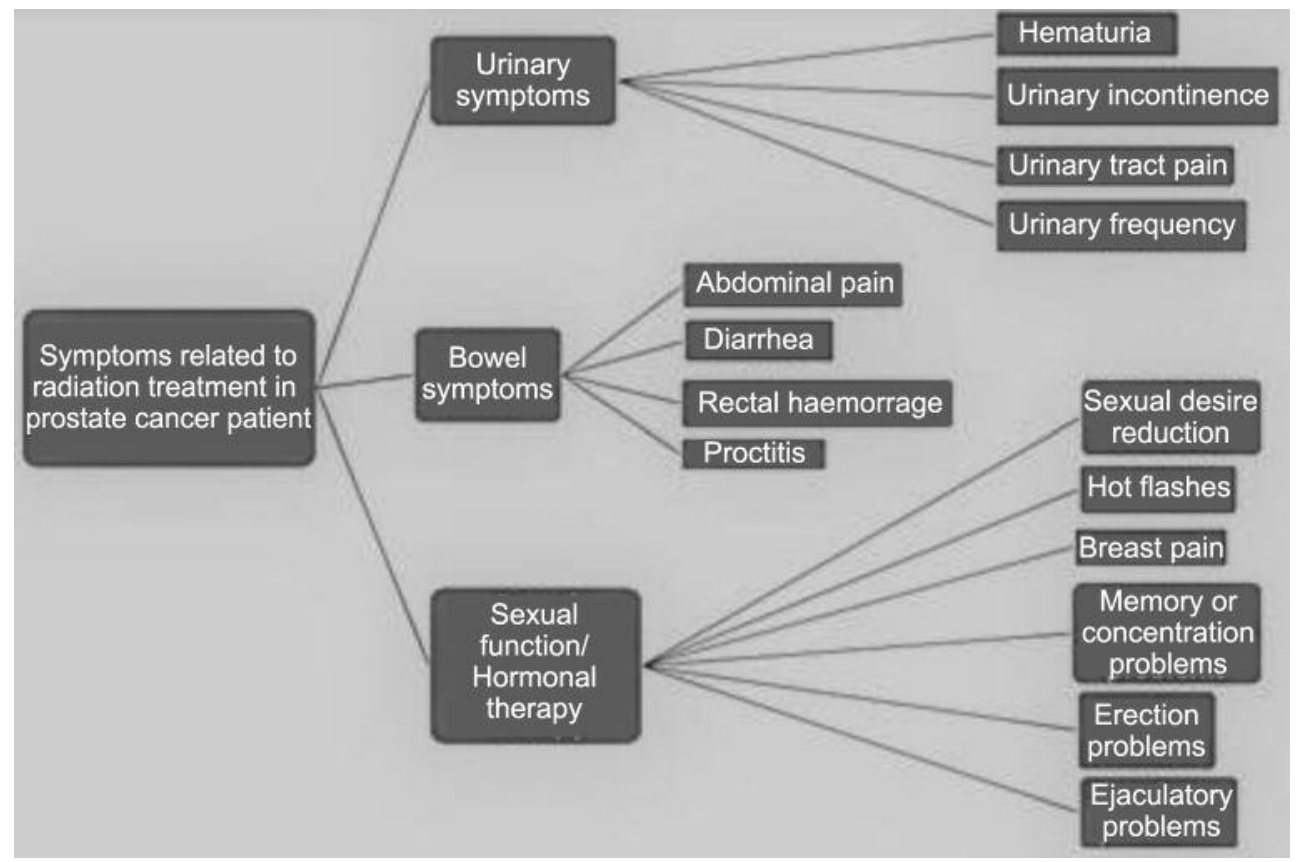

Figure 1. Domains and items included in our electronic PRO tool.

developed an innovative and comprehensive list of items for prostate cancer patients receiving radiotherapy, either as single modality or after surgery, whose main feature is to link the PROs obtained from patients to a wellestablished scale of toxicity. Conclusion: The final validation of this conceptually innovative tool should allow to overcome the differences present in literature and to help both patients and physicians to reduce, and possibly prevent, side effects during and after radiotherapy with a potential improvement in terms of QoL.

\section{7}

\section{PREOPERATIVE IMPACT OF ALBUMIN TO FIBRINOGEN RATIO (AFR) ON ONCOLOGICAL OUTCOMES IN PATIENTS WITH MUSCLE INVASIVE BLADDER CANCER (MIBC) TREATED WITH RADICAL CYSTECTOMY}

Francesco Claps, Matteo Boltri, Luca Ongaro, Francesca Migliozzi, Nicola Pavan, Michele Rizzo, Giovanni Liguori and Carlo Trombetta

Urology Clinic, Department of Medicine, Surgery and Health Science, University of Trieste, Trieste, Italy

Introduction and Objectives: Recent evidence has demonstrated that coagulation is associated with tumor progression in various cancers. Fibrinogen, an essential protein in the coagulation cascade and an acute phase element, plays an important role in cancer: different studies have reported that high preoperative fibrinogen level is associated with tumor development and indicates poor prognosis in some malignancies. Moreover, it is known that malnutrition is a predictor of progression in several types of cancer and albumin is a commonly used index in clinical practice to assess nutritional status. The aim of the study was to investigate the ability of the albumin to fibrinogen ratio (AFR) to predict survival outcomes in patients affected by muscle invasive bladder cancer (MIBC) treated with radical cystectomy (RC). Materials and Methods: We retrospectively evaluated data from 122 patients who underwent RC for MIBC at our Institute with a minimum follow-up of 12 months. AFR score was calculated based on the serum albumin concentration and plasma fibrinogen. Patients were classified into two groups: those with low AFR and those with high AFR. Univariate and multivariate analysis were performed. Recurrence-free (RFS) and Overall Survival (OS) rates were calculated and compared between the two groups. To the best of our knowledge no study in literature have combined albumin with fibrinogen in patients with MIBC. Results: A cut-off value to discriminate between low and high AFR score was determined calculating the ROC curve: the area under the curve was 0.78 with an optimal cut-off of 9.53 points. A total of $41(33.6 \%)$ patients had low AFR. Distribution of data in low and high AFR groups as mean age at surgery 
$(72.3 \pm 8.8 v s .71 .1 \pm 8.9, p=0.35)$, sex (male $23 v s .44$ and female $18 v s .37, p=0.85$ ), presence of high grade disease (39 vs. 72, $p=0.33$ ) lymphovascular invasion (LVI) (16 vs. 27, $p=0.55$ ), adjuvant chemotherapy ( $24 v s .58, p=0.16$ ), mean BMI $(25.2 \pm 3.9 v s .26 .4 \pm 4.3, p=0.17)$ and urinary diversion were not statistically different between two groups. Pathological features as $\mathrm{pT}$ and $\mathrm{pN}$ stages were statistically different ( 0.01 and 0.03 respectively). OS and RFS rates were calculated with a mean follow-up greater in high AFR group $(55.2 \pm 39.2 v s .37 .7 \pm 34, p=0.02)$. Results of the multivariate analysis, after adjustment for age, pT, pN, LVI, surgical margins (R) and ASA score, revealed that AFR was an independent predictor of RFS $(\mathrm{HR}=4.21,2.25-7.88 ; p<0.001)$ and $\mathrm{OS}(\mathrm{HR}=1.82,1.01$ $3.32, p<0.001)$. Conclusion: AFR is a potential prognostic predictor of both OS and RFS in patients with MIBC who underwent surgery.

\section{0}

\section{TOXICITY AND OUTCOMES ASSESSMENT OF HYPOFRACTIONATED HELICAL TOMOTHERAPY FOR LOCALIZED PROSTATE CANCER: A SINGLE-CENTER EXPERIENCE}

Francesco Cuccia $^{1}$, Giuseppe Ferrera ${ }^{2}$, Vincenzo Serretta ${ }^{3}$, Gianluca Mortellaro ${ }^{2}$, Francesco Verderame ${ }^{4}$, Antonio Lupo ${ }^{5}$, Giorgia De Gregorio ${ }^{1}$, Emanuele Quartuccio ${ }^{1}$, Vito Valenti ${ }^{1}$, Antonella Tripoli ${ }^{1}$ and Antonio Lo Casto ${ }^{6}$

${ }^{1}$ Department of Radiation Oncology, ARNAS-Civico Hospital, Radiation Oncology School, University of Palermo, Palermo, Italy; ${ }^{2}$ Department of Radiation Oncology, ARNAS-Civico Hospital, Palermo, Italy;

${ }^{3}$ Department of Surgical, Oncological and Oral Sciences, University of Palermo, Palermo, Italy;

${ }^{4}$ Department of Hematology and Oncology, Hospital Vincenzo Cervello, Palermo, Italy; ${ }^{5}$ Department of Urology, ARNAS-Civico Hospital, Palermo, Italy; ${ }^{6}$ Section of Radiology - Di.Bi.Med, Radiation Oncology School, University of Palermo, Palermo, Italy

Aim: Due to the radiobiological characteristics of a tumor with a low $\alpha / \beta$ ratio, thus more sensitive to higher doses per fraction, prostate cancer (PC) represents one of the first neoplasms where the use of hypofractionation took place, allowing to reach a shortening of the overall treatment time and a higher impact on clinical outcomes. Several reports have shown the noninferiority of moderate hypofractionated schedules (2.2-4 Gy per fraction) in terms of toxicity and biochemical control. Here we report our experience of moderate hypofractionated IMRT-

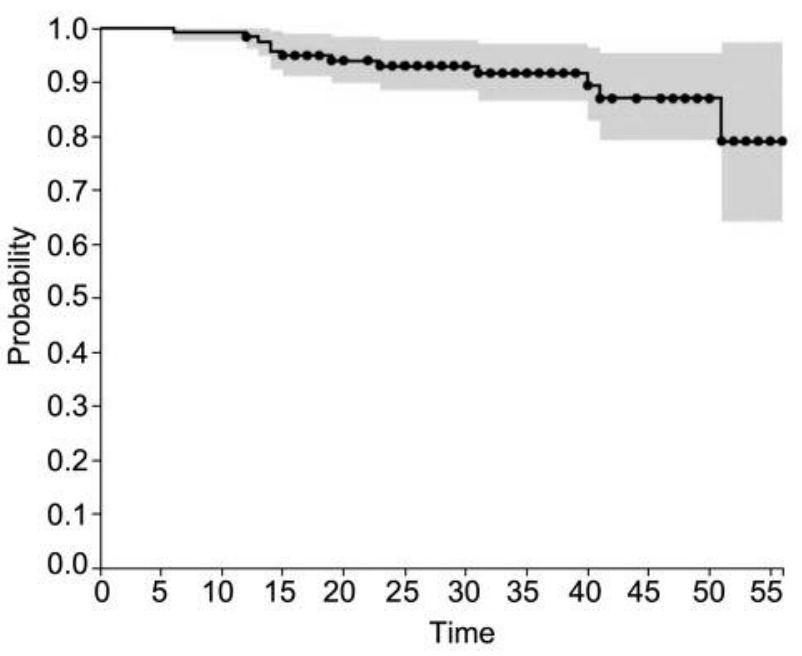

Figure 1. Biochemical failure free survival.

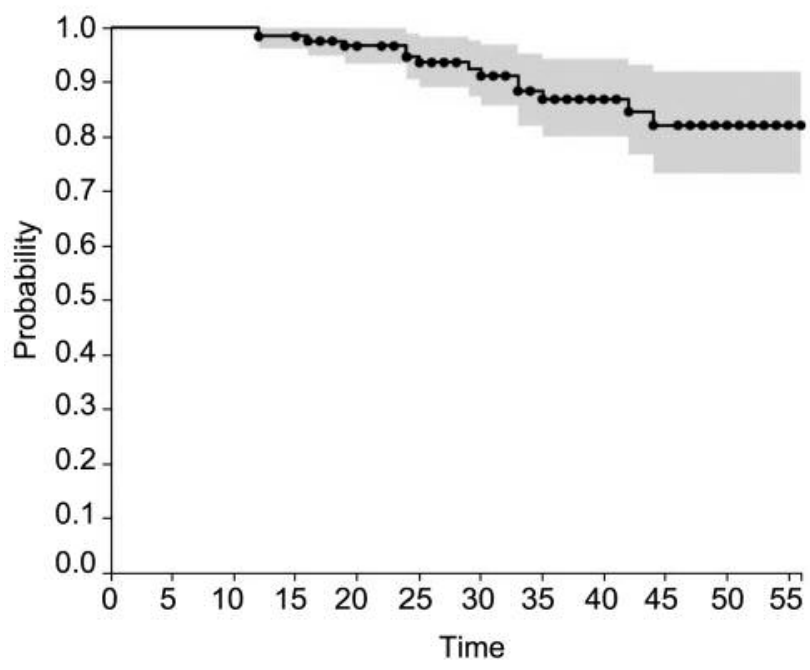

Figure 2. Overall survival.

IGRT with Helical Tomotherapy in the definitive setting of prostate cancer. This study aims to assess toxicity and clinical outcomes. Materials and Methods: From December 2012 to September 2017, 123 patients with prostate cancer were treated with curative intent using hypofractionated Helical Tomotherapy. Median age was 76 years (range $=56-88$ ). $41 \%$ were low risk (LR), $29 \%$ intermediate risk (IR), 30\% high risk (HR); median iPSA was $9.73 \mathrm{ng} / \mathrm{ml}$ (1.35-170). Androgen deprivation therapy was prescribed by the referring urologist according to NCCN recommendations for all intermediate risk and high-risk subjects. For 29 patients with low and intermediate risk disease with a baseline IPSS score $\leq 19$ we adopted a 3 Gy per fraction schedule, delivering in 20 fractions $60 \mathrm{~Gy}$ to the prostate and $54 \mathrm{~Gy}$ to seminal vesicles for IR. All 
the remaining patients received $70 \mathrm{~Gy}$ in 28 fractions to the prostate, using 2.5 Gy per fraction. Through the simultaneous integrated boost technique, in the intermediate risk group seminal vesicles were treated with a total dose of 61.6 Gy using 2.2 Gy fractions; pelvic lymph nodes irradiation in conventional fractionation was added for all the high-risk cases with a total dose of $50.4 \mathrm{~Gy}$. Image guidance was performed with daily megavoltage computed tomography (MVCT) prior to every fraction in order to check setup accuracy and to verify adequate bladder filling and rectal emptying. Toxicity was prospectively evaluated using Common Terminology Criteria for Adverse Events (CTCAE) V4.0; biochemical failure was defined following Phoenix definition of Nadir PSA value $+2 \mathrm{ng} / \mathrm{ml}$. Kaplan-Meier analyses were conducted for Overall Survival (OS) and biochemical failure-free survival (BFFS) estimates. Results: Median follow-up was 33 months (range 12-56). Acute G1 and G2 gastrointestinal (GI) toxicity rates were $20.3 \%$ and $11 \%$; acute G1 and G2 genitourinary (GU) were $47 \%$ and $10.5 \%$; no $\geq \mathrm{G} 3$ was detected. 1-year GI and GU rates were respectively G1 in $18.6 \%, \mathrm{G} 2$ in $3.2 \%, \mathrm{G} 1$ in $19.5 \%$ and $\mathrm{G} 2$ in $2.4 \%$. At two years $(\mathrm{n}=102) \mathrm{GI}$ adverse events rates were $\mathrm{G} 1$ in $\%, \mathrm{G} \geq 2$ in $6.8 \%$, including four cases of $\mathrm{G} 3$ rectal bleeding; for GU toxicity we observed G1 in $10.7 \%, \mathrm{G} \geq 2$ in $2 \%$ (one $\mathrm{G} 3$ urethral stenosis). At 3 years $(n=63)$ toxicity rates were $\mathrm{G} 1$ in $14.2 \%$ and $\mathrm{G} 2$ in $8 \%$, while for GI we observed G1 in $14.7 \%$ and G2 in 3\%, no G3 occurred. After four years $(n=24)$ we observed G1 in $4.76 \%$ for both GI and GU. Most common acute and late adverse events were respectively dysuria and urgency for GU and diarrhea and proctitis for GI. At the time of final analysis, twelve patients experienced a biochemical relapse, including 3 nodal recurrences, successfully treated with stereotactic body radiotherapy, and 2 bone metastases who underwent palliative RT, resulting in 2- and 3-yrs BFFS rates of $93 \%$ and $90.4 \%$ respectively. Three- and 4-yrs OS rates were $90 \%$ and $88 \%$, respectively (Figures 1 and 2). Conclusion: Our series supports the moderate hypofractionated schedule as a well tolerated regimen reporting excellent rates of acute and late toxicity. As regards biochemical control rates, our results are comparable to other experiences of hypofractionation for prostate cancer available in literature.

\section{1 \\ HYPOFRACTIONATED HELICAL TOMOTHERAPY FOR PROSTATE CANCER IN THE POST-OPERATIVE SETTING: A SINGLE-CENTER EXPERIENCE}

Francesco Cuccia $^{1}$, Giuseppe Ferrera ${ }^{2}$, Vincenzo Serretta ${ }^{3}$, Gianluca Mortellaro ${ }^{2}$, Francesco Verderame ${ }^{4}$,

Antonio Lupo ${ }^{5}$, Giorgia De Gregorio ${ }^{1}$, Emanuele Quartuccio ${ }^{1}$, Vito Valenti ${ }^{1}$,

Nicoletta Luca ${ }^{1}$ and Antonio Lo Casto ${ }^{6}$
${ }^{1}$ Department of Radiation Oncology, ARNAS-Civico

Hospital, Radiation Oncology School,

University of Palermo, Palermo, Italy;

${ }^{2}$ Department of Radiation Oncology,

ARNAS-Civico Hospital, Palermo, Italy;

${ }^{3}$ Department of Surgical, Oncological and Oral

Sciences, University of Palermo, Palermo, Italy;

${ }^{4}$ Department of Hematology and Oncology,

Hospital Vincenzo Cervello, Palermo, Italy;

${ }^{5}$ Department of Urology, ARNAS-Civico

Hospital, Palermo, Italy;

${ }^{6}$ Section of Radiology-Di.Bi.Med, Radiation Oncology

School, University of Palermo, Palermo, Italy

Introduction: The use of hypofractionated radiotherapy currently represents a standard for the treatment of prostate cancer in the definitive setting, since several experiences reported excellent outcomes in terms of toxicity and biochemical control. Conversely, few data are available in literature about the use of hypofractionation in the postoperative patient. This is a retrospective mono-institutional analysis of a series of 85 patients with prostate cancer who underwent a moderate post-operative hypofractionated radiotherapy delivered by Helical Tomotherapy (HT). The primary endpoint was acute and late toxicity assessment and the secondary endpoint was early biochemical control. Materials and Methods: From April 2013 to November 2017, 85 consecutive patients with median age $=68$ (range, 54-84) received adjuvant $(n=43)$ or salvage $(n=42)$ moderate hypofractionated radiotherapy performed with Helical Tomotherapy. Adjuvant treatment was administered within 6 months after surgery for patients with PSA $\leq 0.2 \mathrm{ng} / \mathrm{ml}$ in presence of adverse pathological features like extracapsular extension, seminal vesicles invasion, positive margins, or lymph nodal involvement. Salvage therapy was delivered 6 months after surgery with $P S A \geq 0.2 \mathrm{ng} / \mathrm{ml}$ or for patients with persistent post-surgery PSA. Our moderate hypofractionation schedule consisted of a total dose of $63.8 \mathrm{~Gy}$ (EQD2=67.4 Gy) delivered to prostate bed using 2.2 Gy fractions. For patients with pathological adverse features $[\mathrm{pN}+$, inadequate lymph nodal dissection ( $<10$ nodes), and/or Gleason Score $>8$ ] pelvic lymph nodes irradiation with conventional fractionation was indicated in $64 \%$ of cases for a median dose of $49.3 \mathrm{~Gy}$ (range, 48-55.1 Gy). Both for planning CT scan and for treatment, all patients were educated to rectal emptying and bladder filling with the assumption of $500 \mathrm{ml}$ of water 30 minutes prior to every fraction. During the radiotherapy course, daily image guidance was performed with megavoltage computed tomography scan to verify setup accuracy and patients' preparation. Concurrent androgen deprivation therapy was administered according to the discretion of the referring urologist, in $44 \%$ of patients. Acute and late genitourinary (GU) and gastrointestinal (GI) toxicity 


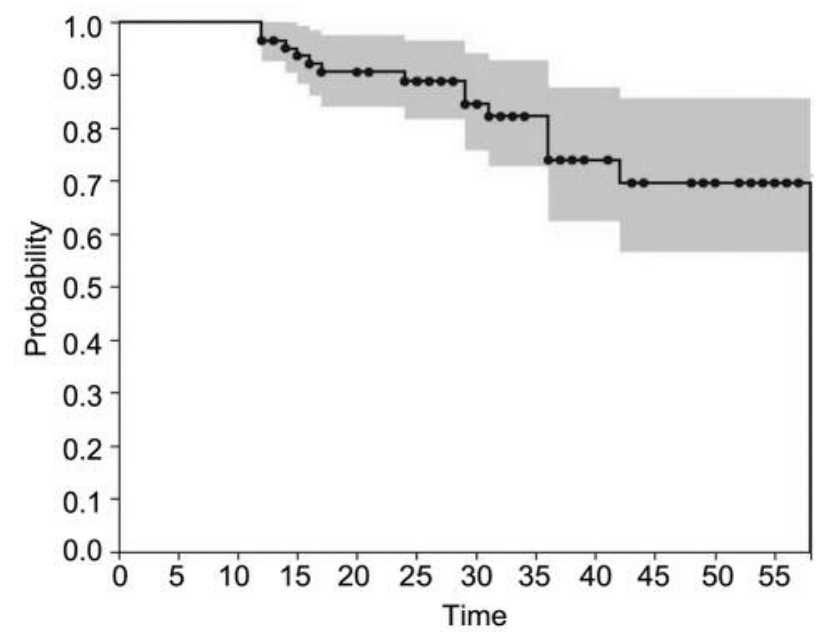

Figure 1. Biochemical control in the entire population.

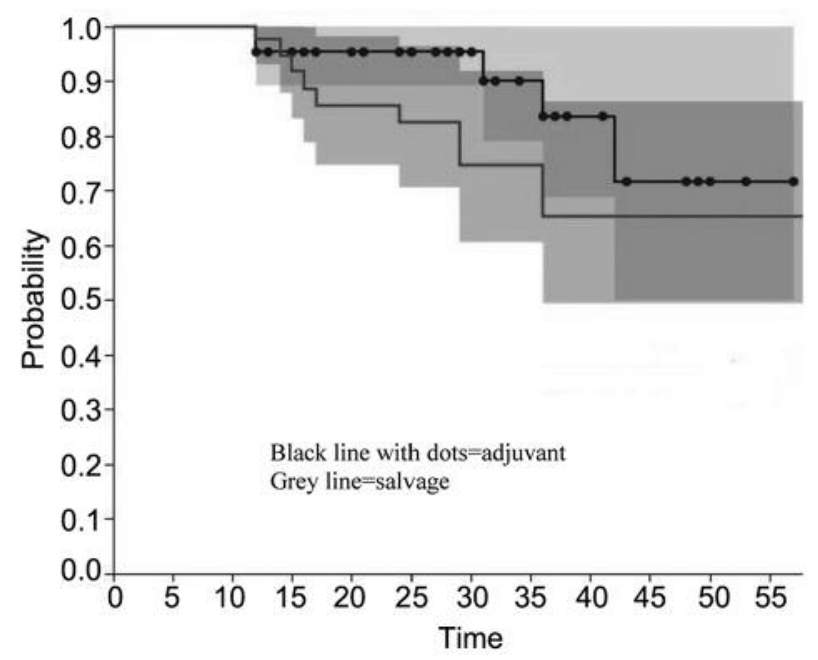

Figure 2. Biochemical control in adjuvant and salvage cohorts.

evaluation was conducted basing on the Common Terminology Criteria for Adverse Events, v4.0 (CTCAE 4.0). Biochemical progression was defined as any PSA level rise of $\geq 0.2$ or more above the post-radiotherapy nadir. Chi-squared tests were applied for statistical analysis assuming $p$-values $\leq 0.05$ as significant. Survival estimates were performed with Kaplan-Meier method and log-rank test. Results: All patients completed the treatment without any interruption. Acute GU toxicities were as follows: G1 in $44 \%$ and G2 in $5.8 \%$, detecting no $\mathrm{G} \geq 3$ events. For GI toxicity, we recorded $\mathrm{G} 1$ in $33 \%$ of patients and G2 in $22.3 \%$. With a median follow-up of 28 months (range, 12-58), we detected G2 GI late toxicity in $5.8 \%$, and $\mathrm{G} \geq 2 \mathrm{GU}$ late toxicity in $4.7 \%$, including 2 patients who underwent surgical incontinence correction, respectively after 24 and 36 months from the end of treatment. Most common acute side effects were urinary tract pain and diarrhea, while, concerning for late adverse events, proctitis and urgency were the most frequently observed. At statistical analysis, acute $\mathrm{GI} \geq 2$ toxicity and diabetes were found to be predictive of late $\mathrm{GI} \geq 2$ toxicity ( $p=0.04$ and $p=0.0019$ ), no other correlations were observed. Actuarial 2- and 3-years biochemical relapse free survival (bRFS) were respectively $88.5 \%$ and $73.7 \%$ for the entire population (Figure 1). In a subgroup evaluation (Figure 2), a statistical significance in terms of biochemical control between adjuvant and salvage patients failed to be found $(p=0.23)$. All patients are alive except one who died because of cerebrovascular disease, resulting in 2- and 3-years overall survival rates of both 97.7\%. Conclusion: In the present experience, our schedule of moderate hypofractionated post-operative radiotherapy with Helical Tomotherapy collected mild acute and late toxicities, either lower or equivalent compared to other series. Also, in terms of biochemical control, our study reports rates in agreement with literature data. A longer follow-up is advocated for a further evaluation of this analysis.

\section{3}

\section{IS THERE DECISIONAL CONFLICT ON TREATMENT OPTIONS ONCE MEN CHOOSE ACTIVE SURVEILLANCE? THE ROLE OF THE MULTIDISCIPLINARY SETTING}

Paola Dordoni ${ }^{1}$, Fabio Badenchini ${ }^{1}$, Letizia De Luca ${ }^{1}$, Barbara Avuzzi $^{2}$, Mario Catanzaro ${ }^{3}$, Simona Donegani ${ }^{1}$, Teresa Di Florio ${ }^{1}$, Alberto Macchi ${ }^{3}$, Tiziana Magnani ${ }^{1}$, Cristina Marenghi ${ }^{1}$, Sara Morlino ${ }^{2}$, Barbara Noris Chiorda ${ }^{2}$, Tiziana Rancati ${ }^{1}$, Silvia Stagni ${ }^{3}$, Antonio Tesone ${ }^{3}$, Sergio Villa ${ }^{2}$, Tullio Torelli ${ }^{3}$, Nicola Nicolai ${ }^{3}$, Roberto Salvioni ${ }^{3}$ and Riccardo Valdagni ${ }^{4}$

${ }^{1}$ Prostate Cancer Program, Fondazione IRCCS Istituto Nazionale dei Tumori, Milan, Italy; ${ }^{2}$ Division of Radiotion Oncology 1, Fondazione IRCCS Istituto Nazionale dei Tumori, Milan, Italy; ${ }^{3}$ Division of Urology, Fondazione IRCCS Istituto Nazionale dei Tumori, Milan, Italy; ${ }^{4}$ Department of Oncology and Hemato-oncology, University of Milan, Radiation Oncology 1, Prostate Cancer Program, Fondazione IRCCS Istituto Nazionale dei Tumori, Milan, Italy

Background: The diagnosis of low-risk prostate cancer (PCa) implies understanding the nature of the disease and facing the possibility of being offered more than one options, including Active Surveillance (AS). AS is a management strategy for men diagnosed with low or very low risk class, potentially indolent prostate cancer $(\mathrm{PCa})$ proposed in alternative to active 
treatment (surgery, external radiotherapy and brachytherapy). Considering the increasing number of patients diagnosed with $\mathrm{PCa}$, the good preliminary results on efficacy of AS and the possibility of sparing therapy-induced side effects, AS is of growing interest for both patients and clinicians as an adequate alternative to active treatment. In offering more than one options, men may enter a phase of decision making characterized by feeling uncertain about the course of action to be taken. In fact, choice among competing options involves risk, loss, and challenges to personal values. Additionally, men might experience anxiety, distress, beliefs and expectations related to PCa diagnosis itself, which may play an additional role in a patient's treatment decision-making (1). The treatment decision making has often been defined as a simple matter: it means to choose the option that prolongs life. However, in case of more than one treatment, the issue becomes to choose the one that maximizes quality of life. But how can a patient choose the best option? Literature on treatment decision making in PCa is growing and shows the complexity of the decision-making process (for a complete analysis see ref. 2); however, still scarce knowledge is available on the potential decisional conflict that may persist after the decision is taken. Do patients on AS suffer from decisional conflict after the dice is cast? Our study aims to answer this question providing an analysis on decisional conflict experienced by men when entering AS. Understanding the decisional conflict of men who decided to enter AS and who experienced the decision-making phase, may help clinicians and psychologist provide adequate support in case of distress related to uncertainty. Materials and Methods: Data were collected from patients enrolled in the

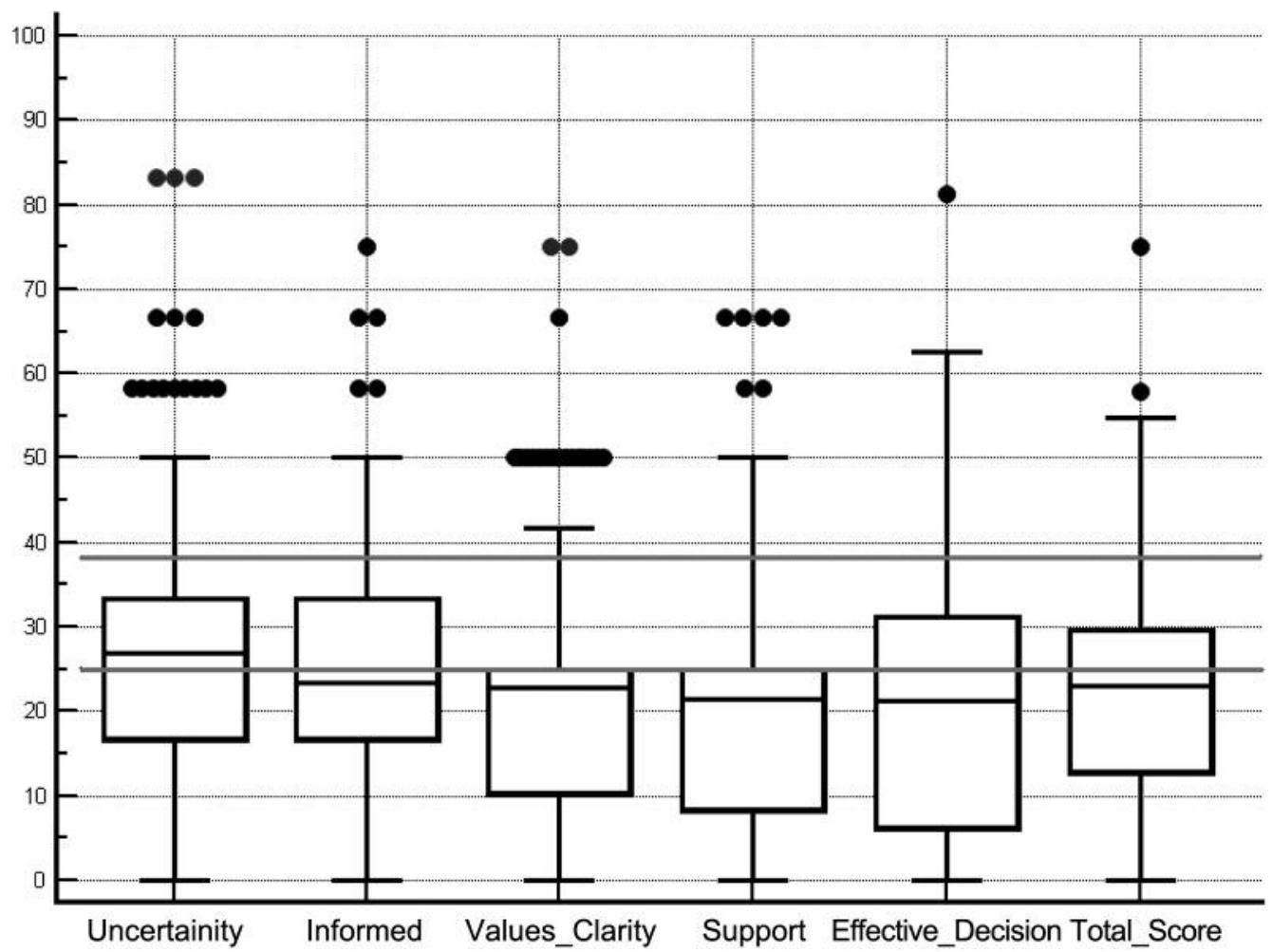

Figure 1. Descriptive results for each DCS subscales. Note: Grey lines show the two thresholds.

Table I. Descriptive analyses of each SCD subscales.

\begin{tabular}{lcccrrr}
\hline & Minimum & Maximum & Mean & Median & SD & 25-75 P \\
\hline Clarity & 0.00 & 75.00 & 22.86 & 25.00 & 15.246 & 10.41 to 25.00 \\
Effective decision & 0.00 & 81.25 & 21.31 & 25.00 & 15.315 & 6.25 to 31.25 \\
Uncertainty & 0.00 & 83.33 & 26.97 & 25.00 & 17.629 & 16.66 to 33.33 \\
Information & 0.00 & 75.00 & 23.44 & 25.00 & 14.645 & 16.66 to 33.33 \\
Support & 0.00 & 66.66 & 21.38 & 25.00 & 15.184 & 8.33 to 25.00 \\
Total score & 0.00 & 75.00 & 23.08 & 25.00 & 13.148 & 12.89 to 29.68 \\
\hline
\end{tabular}


Prostate cancer Research International: Active Surveillance (PRIAS) Quality of Life (QoL) study at entrance in AS protocol (T0). When entering AS, patients had a clinical examination in which the AS protocol was exposed in details. Successively, the psychologist had a brief interview with the patients and proposed the QoL study. Patients were asked to fill in questionnaires among which the Decisional Conflict Scale (DCS), used to explore patients' uncertainty in regards of their decision (score range from 0 to 4 , with 4 indicating certainty). Subscales were: 1) uncertainty (i.e. feeling uncertainty about the choice), 2) informed (i.e. feeling informed about the choice), 3) values clarity (i.e. feeling clear about personal values and risk/side effects of the choice), 4) support (i.e. feeling supported in decision making), and 5) effective decision (i.e. feeling the goodness of the decision). Scores were then recoded (range from 0 -low uncertainty- to 100 -high uncertainty). Descriptive analyses were performed. Scores lower than 25 were associated with implementing decision, while scores higher than 37.5 were associated with decision delay or feeling unsure about the implementation. Results: Between September 2012 and September 2017, 219 patients completed the DCS at the inclusion in AS. Median age was 65 years (range $=45-79$ years), $34.2 \%$ had a job, $38.4 \%$ had a high school degree, $89.6 \%$ had a partner/were married and $28 \%$ had a relevant disease beyond PCa diagnosis. Table I and Figure 1 show descriptive analyses of each subscales of DCS. Findings suggested that $50 \%$ patients had median scores on the threshold of 25 , meaning that they were implementing the decision. More than $75 \%$ patients had scores lower than 37.5 , indicating no delay of the decision or feeling unsure about the implementation. In details, men reported the lowest scores in effective decision $(\mathrm{M}=21.318 ; \mathrm{DS}=15.31)$ and support subscales $(\mathrm{M}=21.38 ; \mathrm{DS}=15.18)$. Discussion and Conclusion: From the moment of diagnosis, patients may deal with the opportunity and at the same time potential burden of choosing among multiple therapeutic/observational strategies that differ in terms of clinical and personal costs and benefits. However, our findings suggested that most men on AS in our Institution were convinced of their choice and having been informed of protocol's details, they also reported to have clear personal values and to know well risks and side effects of the choice they had made. Dealing with the same issue, Vasarainen et al. (3) highlighted the relevant impact of offering AS patients more information and support. They reported on a program in which a specialized prostate cancer nurse functioned as a bridge between patients and urologists. In a similar vein, the fact that men showed to have received enough information about AS and useful support before entering the protocol suggests that adequate information is needed in order to help patient during the decision-making phase. The presence of a multidisciplinary setting, in which a team of psychologists might help patients avoid feeling confused or distressed, is essential in order to support men in this process. In our
Institution, men received information on the opportunity of AS upfront, during the multidisciplinary first consultation in which a urologist, a radiation oncologist, a psychologist, and an ondemand medical oncologist are present) or the first urologic/radiotherapeutic visit. After the decision-making phase (2), when entering AS a new appointment with a medical oncologist participating in AS protocols is scheduled aimed to give the patient all information he needs and to answer his questions. Immediately after the visit the psychologist conducts a brief meeting aimed to value patients' psychological health (i.e. anxiety and coping styles). The psychologist can take care of the patient through individual support. Supporting men and their families in the treatment decision making process can be challenging and time-consuming, but in light of our clinical experience and our findings a multidisciplinary management of patients who are eligible for AS might be recommended. In conclusion, our findings support recommendations about the importance of a psychological assessment at the entrance of assessing decisional conflict on AS, so that patients who may feel uncertain and/or distress about choosing AS can receive adequate multidisciplinary support.

1 Bellardita L, Graffigna G, Donegani S, Villani D, Villa S, Tresoldi V, Marenghi C, Magnani $\mathrm{T}$ and Valdagni R: Patient's choice of observational strategy for early-stage prostate cancer. Neuropsychol Trends 12: 107-116, 2012. DOI: 10.7358/neur-2012-012-bell

2 Bellardita L, Dordoni P, De Luca L, Menichetti-Delor JP and Valdagni R: Better informed decision making to optimize patient selection. In: Active Surveillance for Localized Prostate Cancer. Klotz L (eds.). Toronto, Humana Press, Cham., pp 149-167. DOI: 10.1007/978-3-319-62710-6_14

3 Vasarainen H, Lokman U, Ruutu M, Taari K and Rannikko A: Prostate cancer active surveillance and health-related quality of life: results of the Finnish arm of the prospective trial. BJU Int 109(11): 1614-1619, 2012. PMID: 22044485. DOI: $10.1111 /$ j.1464-410X.2011.10677.x.

\section{4 \\ PRESENCE OF UNSAMPLED GLEASON PATTERN 4 CAN BE PREDICTED FROM CHARACTERISTICS OF ADJACENT GLEASON PATTERN 3}

$\underline{\text { Alessandro Caputo }}^{1}$, Domenico Memoli ${ }^{2}$, Pasquale Cretella ${ }^{1}$, Pio Zeppa ${ }^{1}$ and Antonio D' Antonio ${ }^{2}$

${ }^{1}$ Department of Medicine and Surgery, University of Salerno, Baronissi, Italy; ${ }^{2}$ Unit of Pathology, University Hospital "S. Giovanni di Dio e Ruggi D’Aragona", Salerno, Italy

Introduction: Prostate biopsies undergrade $25 \%$ to $50 \%$ of prostate cancers, delaying definitive treatment by up to 3 years 

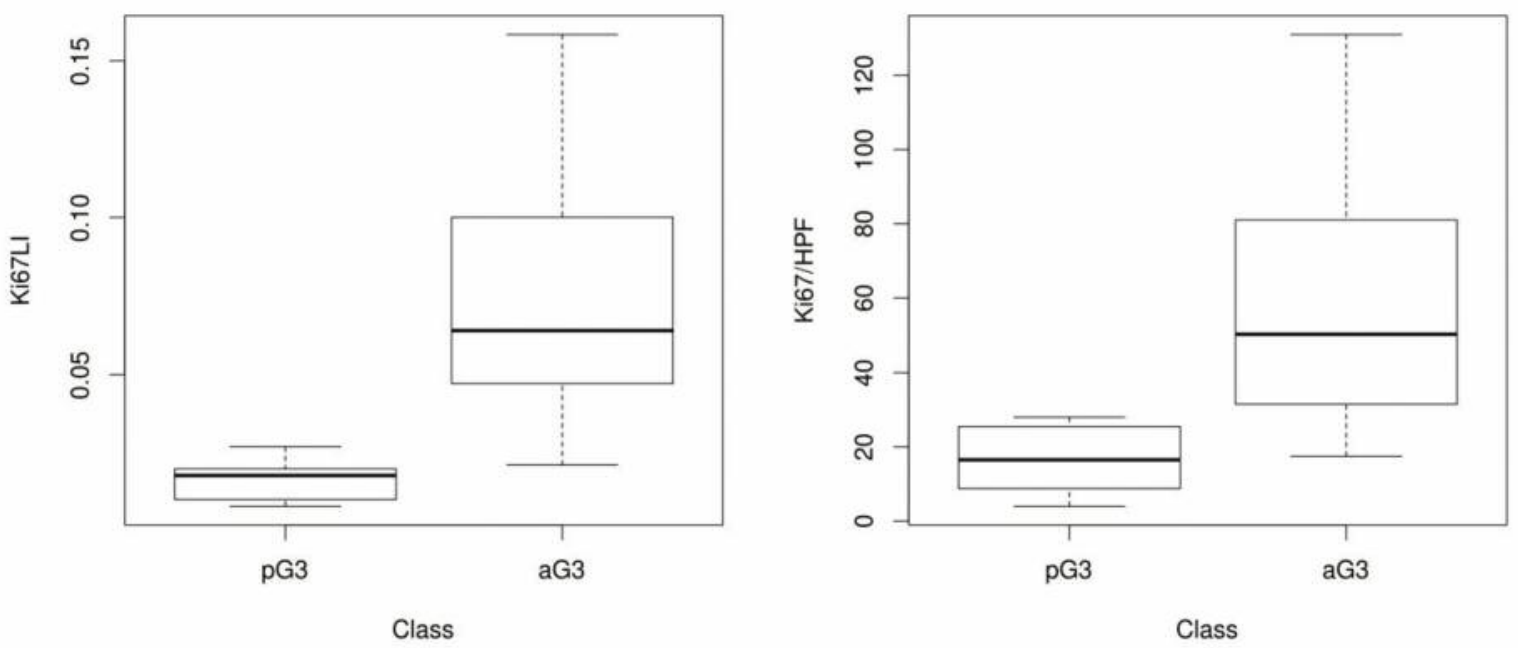

Figure 1. Left: box plot of the Ki67 labelling index (Ki67LI) in pG3 and aG3. Right: box plot of Ki67 positivity per high-power field (Ki67/HPF) in $p G 3$ and $a G 3$.

(1). A cause of undergrading is the partial sampling inherent in biopsies: if a biopsy samples a low-grade (Gleason pattern 3) area but misses an adjacent high-grade (Gleason pattern 4 or 5) focus, the overall grade of the tumor will be underestimated. In other words, when faced with a prostate biopsy that appears to be Gleason $3+3=6$, the pathologist may unknowingly be looking at a part of a higher-grade tumor that has been sampled only partially. However, not all Gleason pattern 3 prostate cancers are equal: mounting evidence hints at the possibility that pure Gleason pattern 3 (pG3) is biologically distinct from Gleason pattern 3 associated with higher-grade prostate cancer (aG3). In this study, we used immunohistochemistry and computer-aided image analysis to compare the expression of Ki67, cyclin D1, MYC, and p53 between foci of aG3 and pG3, in search of a reliable and cost-effective marker to distinguish them. When a biopsy only shows Gleason pattern 3 prostate cancer $(3+3=6)$, having such a marker would allow us to distinguish the true $3+3=6$ from the false ones that only *appear* to be $3+3=6$ because of a sampling error. This would dramatically improve the diagnostic performance of prostate biopsies and the management of early prostate cancer. Materials and Methods: Our pathology database was searched for cases of prostatectomy or cystoprostatectomy with a diagnosis of prostatic adenocarcinoma. The corresponding slides were retrieved from our archive and reviewed to search for: - foci of pure Gleason 3 prostate cancer (pG3), consisting exclusively of Gleason pattern 3, without any adjacent pattern 4 or 5; - foci of Gleason 3 adjacent to higher grade prostate cancer (aG3), where pattern 3 glands were spatially adjacent to Gleason 4 or 5 glands. For each focus, the slide with the most Gleason pattern 3 was chosen and its relative formalin-fixed paraffin-embedded tissue block was retrieved to cut additional $3 \mu \mathrm{m}$-thick sections for immunohistochemical analysis. We used four antibodies: Ki67, Cyclin D1, p53, and MYC. All immunohistochemical scoring was done for the Gleason 3 component of the focus: only cells belonging to a Gleason pattern 3 gland were considered. Stromal cells, benign glands, PIN and Gleason patterns 4 and 5 were ignored. For each focus, Ki67 was evaluated using two indices: the percent of positive cells (labelling index, Ki67LI) and the number of positive cells per high-power field (Ki67/HPF, with 1 $\mathrm{HPF}=0.36 \mathrm{~mm}^{2}$ ). For each focus the regions with the highest density of positive cells were identified and captured in digital images (mean: 3.43 images per focus; each image represented $1 \mathrm{HPF}$ ). Any nuclear DAB staining was considered positive. The images were analyzed using QuPath (a free and opensource computer program for digital pathology). For each image, "Positive cell detection" was used to recognize each nucleus, and then a classifier was trained to distinguish positive, negative and stromal cells. The number of positive cells was also counted manually, on the same images, using the Multi-point tool in ImageJ. Each positive cell was clicked on in turn, while the software kept the tally and placed a marker on each counted cell to avoid double-counting. The agreement between manual and computer-aided counting of positive cells was excellent, with an intraclass correlation coefficient of 0.946 (calculated with the *icc* function of the *irr* package in $\mathrm{R}$ ). Finally, for each focus the Ki67LI was calculated as total positive cells divided by total prostate cancer cells, and the Ki67/HPF was calculated as total positive cells divided by the number of images evaluated for that focus. The other markers were evaluated by analyzing the slides directly under the microscope. A focus was considered positive for Cyclin D1 if at least $20 \%$ of its cells displayed any nuclear DAB staining, while p53-positivity required nuclear staining of moderate or high intensity in at least $20 \%$ of the cells. MYC was classified 


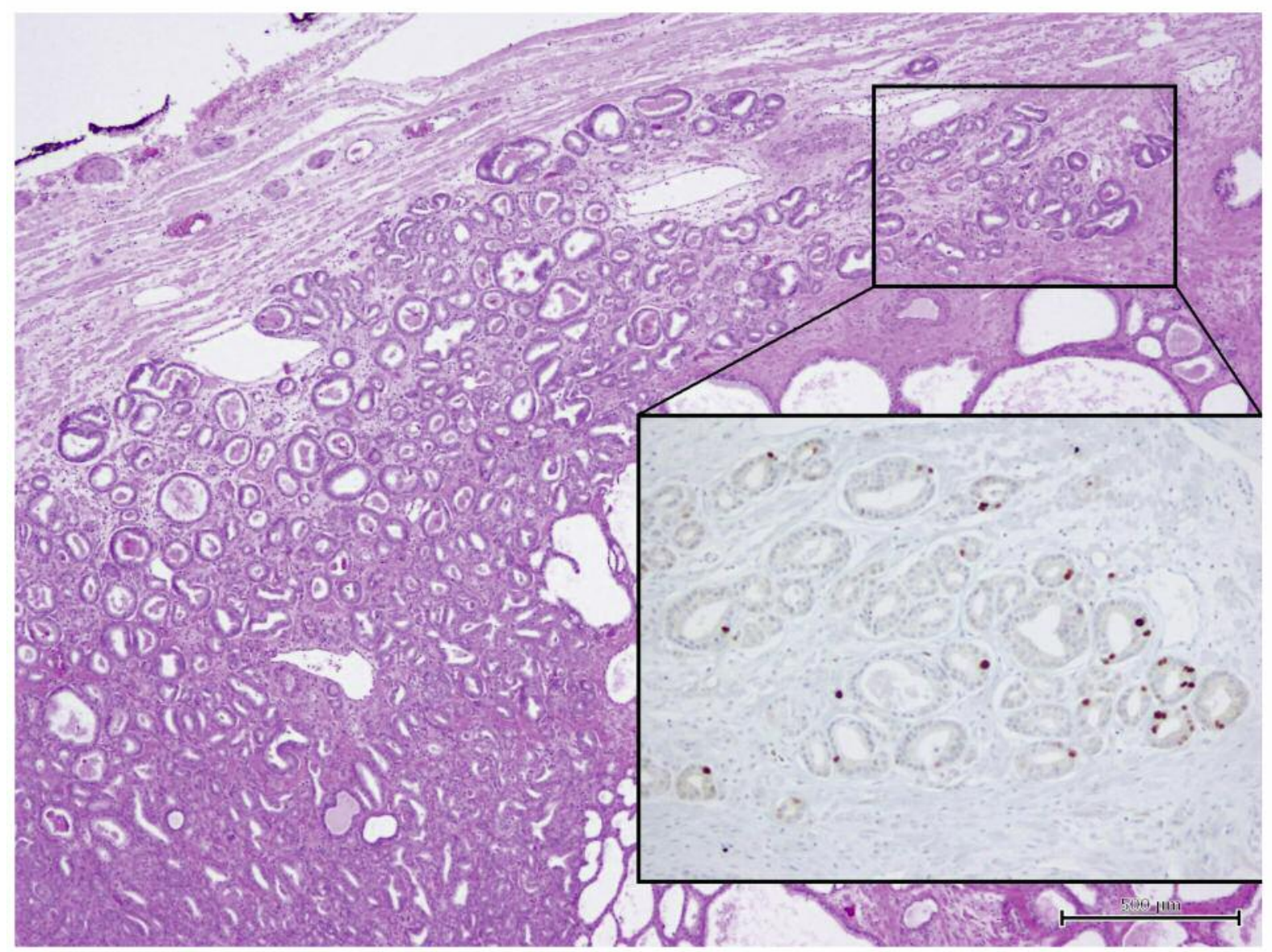

Figure 2. Gleason pattern 3 prostate cancer (upper right) adjacent to pattern 4 (lower left). Hematoxylin and eosin, $40 \times$. Inset: Ki67 immunohistochemistry of the marked region. 3-3' diaminobenzidine, 200x. The region of pattern 3 furthest (>5 mm) from Gleason pattern 4 still showed Ki67LI=9.2\% and Ki67/HP=60.

in three categories: absent/weak, moderate/strong cytoplasmic but not nuclear positivity in at least $20 \%$ of cells, and moderate/strong nuclear positivity (regardless of cytoplasmic positivity) in at least $20 \%$ of cells. Statistical analysis: Statistical analysis was performed to evaluate the ability of each of the four markers to distinguish pG3 from aG3. Fisher's exact test was used for binary variables (p53, MYC, Cyclin D1), while Student's $t$-test was used for Ki67LI and Ki67/HPF. All statistical analyses were performed using $\mathrm{R}$ version 3.5.1. A $p$-value lower than 0.05 was considered statistically significant for all tests. Results: A total of 8 pG3 and $22 \mathrm{aG} 3$ foci were included in the present study. The average Ki67LI was $6.03 \pm 4.20 \%$ (pG3: $1.63 \pm 0.65 \%$; aG3: $7.62 \pm 3.77 \%$ ). Ki67LI is significantly different between pG3 and aG3 $(p<0.000001)$. The average Ki67/HPF was 48.6 \pm 34.8 (pG3: 16.7 $\pm 9.61 ;$ aG3: $60.2 \pm 33.4)$. The difference between $\mathrm{pG} 3$ and aG3 is statistically significant $(p<0.00001)$. The expression of p53, Cyclin D1 and MYC did not significantly differ between pG3 and aG3. Cyclin D1 positivity appeared to be significantly associated with higher Ki67/HPF ( $p=0.01)$. An association with higher Ki67LI was also observed, but it did not reach the threshold for statistical significance $(p=0.077)$. Discussion and Conclusion: We studied the expression of Ki67, p53, Cyclin D1 and MYC in foci of pure Gleason pattern 3 prostate cancer (pG3) and compared them with those in Gleason pattern 3 associated with high-grade tumor (aG3), to find out whether one of these markers could be able to distinguish pG3 from aG3. The Ki67 labelling index (Ki67LI) appears to be able to reliably distinguish pG3 from aG3 (Figure 1). Using 3.0\% as a cutoff, Ki67LI is able to distinguish pG3 from aG3 with $95 \%$ sensitivity and $100 \%$ specificity. The number of Ki67-positive cells per high power field (Ki67/HPF) was also able to distinguish pG3 from aG3 (using 30 as cutoff, the sensitivity was $86 \%$ and the specificity was $100 \%$, whereas with 15 as cutoff, the sensitivity was $100 \%$ at the cost of only $50 \%$ specificity) (Figure 1). Our findings on Ki67LI are in line with those present in the literature (2). Moreover, it is possible that the worse prognosis of Gleason $3+3=6$ prostate cancer with 
Ki67LI $>10 \%$ observed by some authors is due to the fact that Gleason pattern 3 with high Ki67LI is actually aG3, and thus it is associated with (unsampled) Gleason pattern 4 (3). It is interesting to note that the alterations characteristic of aG3 are not limited to the Gleason 3 glands directly adjacent to patterns 4 or 5 , but they extend to the whole nodule of pattern 3 . In one of our cases we observed a focus of Gleason pattern 3 which on one side was adjacent to pattern 4 , while on the other it extended for $5 \mathrm{~mm}$ along the prostate margin. The region of this pattern 3 nodule furthest $(>5 \mathrm{~mm}$ ) from Gleason pattern 4 still showed Ki67LI=9.2\% and Ki67/HPF=60 (Figure 2). The absence of statistically significant differences in the expression of Cyclin D1, MYC and p53 between pG3 and aG3 is in line with the literature, as is our finding regarding the differences in Ki67 expression between Cyclin D1-positive and -negative cases of prostate cancer. Calculating the Ki67LI is relatively fast using appropriate software. However, it does require taking digital pictures and then analyzing them, which can quickly become a burden in a regular hospital without experience in digital pathology. For this reason, we demonstrated that an index as simple as the number of Ki67-positive cells per high power field can be used as a first-line test: cases with $\mathrm{Ki67/HPF}<15$ are very likely to be pG3 ("true" $3+3=6$ ), whereas cases with $\mathrm{Ki67/HPF}>30$ are very likely to be aG3 ("false" $3+3=6$, with unsampled pattern 4 or 5 ). Only the grey area in between would require the calculation of the Ki67LI To sum up, this proof-of-concept study confirms that pG3 and aG3 are two distinct entities, and is the first to our knowledge to translate this concept to the clinical practice by identifying an affordable and straightforward method to distinguish these two entities. Such a method is able to predict which patients with a Gleason $3+3=6$ prostate biopsy are truly $3+3=6$ (pG3) and which *appear* to be $3+3=6$ due to a sampling error, but actually harbor a prostate cancer of Gleason score at least equal to 7 , and thus would likely benefit from early radical treatment more than from active surveillance. Using Ki67/HPF and $\mathrm{Ki67LI}$ it is possible to distinguish these patients with $>95 \%$ specificity and sensitivity. Should our observations be validated by future studies, the diagnostic performance of prostate biopsies would noticeably improve. Fewer patients with unsampled high-grade prostate cancer would be erroneously enrolled in active surveillance protocols. So not only would they not undergo the additional biopsies that within 2-3 years would eventually sample the Gleason pattern 4, but also their radical surgical treatment would not be delayed so much.

1 Epstein JI, Feng Z, Trock BJ and Pierorazio PM: Upgrading and downgrading of prostate cancer from biopsy to radical prostatectomy: incidence and predictive factors using the modified Gleason grading system and factoring in tertiary grades. Eur Urol 61(5): 1019-1024, 2012. PMID: 22336380. DOI: 10.1016/j.eururo. 2012.01 .050
$2 \mathrm{Fu}$ L, Hwang M, Adeniran AJ and Humphrey PA: Proliferation index of different Gleason pattern 4 histomorphologies and associated pattern 3 adenocarcinoma of the prostate. Hum Path 70: 1-5, 2017. PMID: 28666926. DOI: 10.1016/j.humpath.2017.06.007

3 Zellweger T, Günther S, Zlobec I, Savic S, Sauter G, Moch H, Mattarelli G, Eichenberger T, Curschellas E, Rüfenacht H, Bachmann A, Gasser TC, Mihatsch MJ and Bubendorf L: Tumour growth fraction measured by immunohistochemical staining of Ki67 is an independent prognostic factor in preoperative prostate biopsies with small-volume or lowgrade prostate cancer. Int J Cancer 124(9): 2116-2123, 2009. PMID: 19117060. DOI: $10.1002 / \mathrm{ijc} .24174$

\section{5}

\section{HELICAL TOMOTHERAPY IN THE TREATMENT OF UNFIT BLADDER CANCER PATIENTS: A MONO-INSTITUTIONAL STUDY (2011-2017)}

Francesco Pastore, Annaisabel Rese,

Ferdinando Francomacaro, Fabrizio Cammarota, Gianluca Ametrano, Diego Toledo and Vincenzo Iorio

\section{Department of Radiation Oncology, EMICENTER,} Mondragone, Italy

Aim: to evaluate the use of Helical Tomotherapy (HT) in the treatment of bladder cancer. The HT system employs a compact $6 \mathrm{MV}$ Linac-based on CT ring gantry to rotationally deliver intensity modulated fan beams. Patients are translated through-out the gantry on a treatment couch, resulting in helical irradiation geometry. The HT unit also contains a mega-voltage CT detector array located opposite the radiation source for pre-treatment verification, allowing accurate repositioning. This technique permits to precisely target tumors while minimizing impact on surrounding healthy tissue: this could be very interesting for unfit bladder patients, to reduce GU and GI toxicity. Patients and Methods: A total of 31 patients (age: 54-87) with bladder cancer were treated in our institution from 2011 to 2017 with HT. None of these patients were fit for surgical indication due to concomitant medical conditions. None of these patients had concomitant chemotherapy due to medical conditions. 18/31 (58\%) of these patients had positive lymphonodes. CT axial scanning was performed at $5 \mathrm{~mm}$ intervals: for this purpose, a CT Multislice GE Healthcare Discovery 590HT was used. The radiation oncologists contoured the volumes of interest (CTV) according to the RTOG guidelines. The planning target volume (PTV) was generated from the CTV volume by adding a $3 \mathrm{~mm}$ margin in all directions. Accurate delineation of organ at risk was performed. In these patients, we used several radiotherapy schedules, according to volume and site of cancer and considering performance status of the patients. Treatment 
plans were evaluated on a dedicated TPS. Results: A total of 27 out of the 31 of these patients ended scheduled radiotherapy: 3 patients ended early treatment for cardiac condition, 1 for diabetes complications. Median follow up was about 11 months (range 2-41). All patients had acute GU toxicity: 17 patients $(63 \%)$ had G1, 7 patients $(25 \%)$ had G2 and 3 patients (12\%) had G3. Late GU toxicity was seen in 7 patients (26\%) as G1 and in 2 patients (7\%) as G2. 15 patients (55\%) had GI toxicity: 13 patients $(86 \%)$ had G1, 2 patients (14\%) had G2. Late GI toxicity was seen in 4 patients $(15 \%)$ as G1. OS was $79 \%$ at 1 year, $66 \%$ at 2 years and $41 \%$ at 3 year. Discussion: HT is a safe and feasible technique to treat unfit patients with bladder cancer. All patients had acute GU toxicity, but it was acceptable for the greatest part of them. Acute GI toxicity was acceptable too. Late toxicity, both GU than GI, was never $\geq G 3$. The OS is surely influenced by the poor clinical concomitant conditions of the patients too.

\section{6}

\section{RECURRENT CONTRALATERAL LEIOMYOSARCOMA OF THE SPERMATIC CORD}

Valerio Olivieri ${ }^{1}$, Valentina Fortunati ${ }^{2}$, Luca Bellei ${ }^{1}$, Sandro Guglielmetti ${ }^{1}$, Massimo Massarelli ${ }^{1}$, Massimo Ollino ${ }^{1}$, Emanuele Corongiu ${ }^{3}$, Flavio Forte ${ }^{3}$, Pier Paolo Prontera ${ }^{4}$, Silvia Ferrario ${ }^{5}$ and Scipio Annoscia ${ }^{1}$

${ }^{1}$ Division of Urology, Ivrea Hospital, Ivrea, Italy;

${ }^{2}$ Division of Pathology, Policlinico

Tor Vergata, Rome, Italy;

${ }^{3}$ Division of Urology, Ospedale

"Madre Giuseppina Vannini", Rome, Italy;

${ }^{4}$ Division of Urology, Policlinico Umberto I, Rome, Italy;

${ }^{5}$ Radiotherapy, Ivrea Hospital, Ivrea, Italy

Background: Leiomyosarcomas (LMS) are extremely rare neoplasms totally accounting for $8 \%$ of all soft tissue tumors. In contrast to the benign variant of leiomyoma, they are considered as malignant cancers. Taking origin from smooth muscle, they may differently involve many organs but rarely affect spermatic cord or scrotum. Their clinical behavior is highly unpredictable and data in literature are sometimes controversial: to date surgery represents the best option. We report the case of a voluminous recurrent contralateral leiomyosarcoma of the scrotum. Case Report: A 72-year old caucasian male presented to our facility for left scrotum swelling of two years duration which started growing rapidly in last six months. On past anamnesis he suffered from left inguinal hernia and benign prostatic hyperplasia actually in therapy with terazosin. Moreover, five years previously he underwent a radical excision of large right scrotal mass taking origin from spermatic cord: at the same time a radical orchiectomy was also performed due to the macroscopic

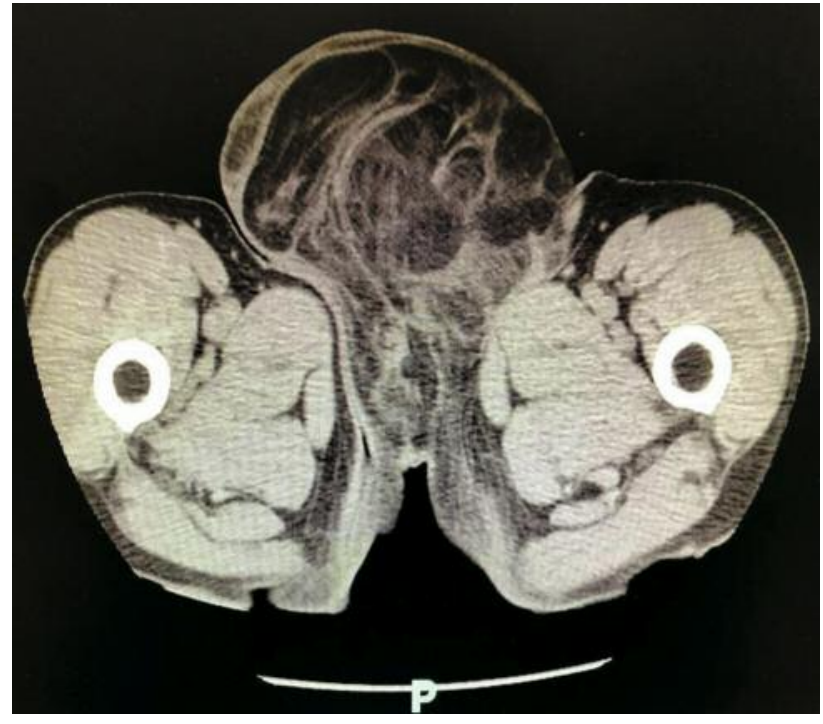

Figure 1. Contrast enhanced CT showing heterogeneous mass totally occupying the scrotum.

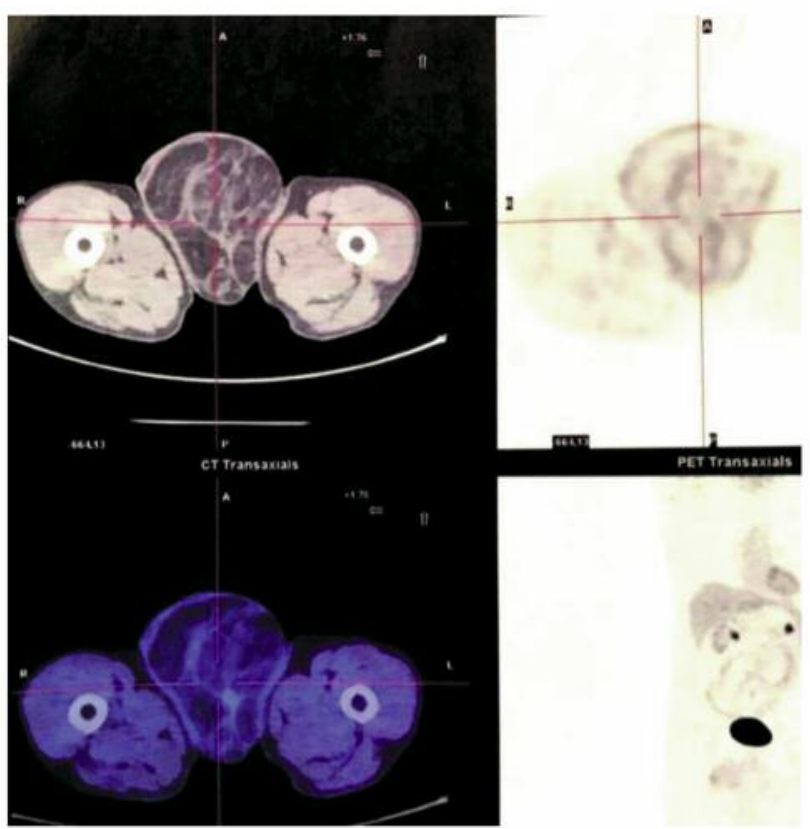

Figure 2. PET exam confirms the absence of enhancement inside the mass.

testicular involvement. In that case pathology report revealed a leiomyosarcoma lipoma-like of the right spermatic cord: according to the Fèdèration Nationale des Centres de Lutte Contre le Cancer (FNCLCC) scoring system and microscopic features, it was classified as low grade (G1). On follow up no signs of recurrent neoplasms were identified. Six months ago, 
the patient noticed an increase in left scrotum volume initially attributed to a worsening of inguinal hernia. On physical examination the scrotum was enlarged, soft and totally occupied by a tender mass. Due to his past medical history, the patient underwent contrast enhanced total body computerized tomography (CT) which revealed a large heterogeneous mass entirely occupying the left inguinal canal and scrotum (Figure 1), probably taking origin from spermatic cord but not involving left testis: no calcifications inside the mass or inguinal lymph nodes enlargement were described nor bone or visceral metastases. Hence in order to exclude also micrometastases, the patient also underwent a positron emission tomography (PET/TC): the examination further confirmed the absence of bone, lymph nodes and visceral metastases (Figure 2). The patient was offered a radical excision of the mass with the intent to save the unilateral testis. During surgical procedure the testis and epididymis were successfully separated from the lesion and the mass was removed alone. Postoperative recovery was uneventful. Results: On macroscopic examination, the neoplasm was soft, of light-yellow appearance and adipose consistency, with a total length of $22 \times 19 \mathrm{~cm}$ and a weight of 2070 grams. According to FNLCC grading score, on this time it was classified as a grade G2 showing the following features: differentiation score 2 and mitotic count score 1 (2/10 HPF). Resection margins were positive for tumor: no lymph-vascular invasion was described (L0; V0) nor necrosis (score 0 ). The immunohistochemistry was positive for desmin, smooth actin, totally confirming histological origin of the lesion. It was diagnosed as recurrent leiomyosarcoma 'lipoma like' of contralateral spermatic cord G2 pT4R1L0V0 cN0 cM0. Patient was referred to regional center for rare tumors in order to evaluate further adjuvant treatments considering the positivity of surgical margins. Discussion and Conclusion: Sarcoma tumors are rare neoplasms totally accounting for $1 \%$ of all human cancers: leiomyosarcoma is a histotype of sarcoma. Pathophysiologic mechanisms involved in its etiology still remain unknown (1). Although it rarely origins from spermatic cord, it is mentioned among scrotal masses of non-testicular origins (2). About incidence, it represents $5-10 \%$ of malignant soft tissue tumours (3). Taking origins from smooth muscle, leiomyosarcoma may potentially occur anywhere. It usually involves different sites and organs as lung, liver, spleen small intestine and stomach. It may also involve pelvic organ like bladder or uterus: spermatic cord origin has been also described in literature but is uncommon and quite rare. Moreover, to our knowledge this represents the first case described in literature about recurrent contralateral leiomyosarcoma of spermatic cord. Its clinical evolution is hardly unpredictable since some of them remain dormant for years while a great many others show an aggressive behavior and may easily spread. Differentiation, mitotic count, necrosis, lymph-vascular invasion and intralesional calcifications represent the main features to evaluate in order to predict its clinical behavior. Clinical presentation is strongly variable and symptoms often depend on location: frequently they may be absent until the lesion reaches a large volume. Computerized tomography (CT) and magnetic resonance (MRI) represent the best diagnostic tools to identify and describe dimensions, location, organs involvement and, whenever present, metastases. Radical surgery seems to be considered the best treatment since many studies in literature suggest its unresponsiveness to radiation and chemotherapeutic regimens. In conclusion, spermatic cord sarcoma represents a rare histologic entity, usually growing as paratesticular mass. Diagnosis is based on CT or MRI since these masses are frequently indolent. Surgery represents the best treatment for these lesions, but whenever not completely removed they can easily recur. Unfortunately, due to the low number of incidences of similar cases, few information is available about treatment of recurrent cases and surgery still remains the single option.

1 Serrano C and George S: Leiomyosarcoma. Hematol Oncol Clin North Am 27: 957-974, 2013. PMID: 24093170. DOI: 10.1016/j.hoc. 2013.07.002

2 Dangle P, Basavaraj D, Bhattarai S, Paul AB and Biyani CS: Leiomyosarcoma of the spermatic cord: case report and literature review. Can Urol Assoc J 1(1): 55-58, 2007. PMID: 18542764

3 Frigerio P, Muruato-Araiza J, Marcos-Morales S, CepedaNieto AC, Berdeal-Fernandez E and Zepeda-Contreras S: Spermatic cord leiomyosarcoma rare case. Urol Case Rep 6: 15-17, 2016. PMID: 27169019. DOI: 10.1016/ j.eucr.2016.01.002

\section{7 \\ STRATIFICATION OF INTERMEDIATE-RISK NON-MUSCLE INVASIVE BLADDER CANCER PATIENTS: IMPLICATIONS FOR ADJUVANT THERAPIES}

Francesco Soria $^{1}$, David D'andrea ${ }^{2}$, Kilian Gust $^{2}$, Paolo Gontero1 and Shahrokh Shariat ${ }^{2}$

${ }^{1}$ Division of Urology, Azienda Ospedaliera Città della Salute e della Scienza di Torino, Università degli

Studi di Torino, Turin, Italy;

${ }^{2}$ Department of Urology and Comprehensive Cancer Center, Medical University of Vienna, Vienna General Hospital, Vienna, Austria

Introduction and Objectives: Due to the high recurrence rate of bladder cancer, many patients are recategorized to harbor intermediate risk non-muscle invasive bladder cancer (NMIBC). The EAU guidelines recommend administration of intravesical 
Bacillus Calmette-Guerin (BCG) followed by a one-year maintenance or induction intravesical chemotherapy for the adjuvant treatment of these patients. Risk-stratification of the heterogeneous cohort of patients with intermediate NMIBC could help select between treatment strategies, as well as facilitate follow-up scheduling. Indeed, a more risk-based approach would usher the age of personalized medicine for intermediate risk NMIBC. Moreover, in case of BCG-shortage, this could help providers in the selection of the patients who could safely receive intravesical chemotherapy without compromising outcomes. The aim of our study was to assess whether patients with intermediate risk NMIBC can be stratified into different risk groups, thereby providing a practical tool for the selection of the optimal adjuvant therapy for the right patient, based on the individualized risk of disease progression. Materials and Methods: We performed a retrospective analysis of patients with intermediate risk NMIBC treated with transurethral resection of the bladder (TURB) and adjuvant intravesical chemotherapy. All patients had pTa low-grade recurrent bladder cancer. Collected variables included age, gender, frequency of recurrence (less or more than one per year), timing of recurrence (early recurrence defined as occurring $<12$ months), size and focality. A multivariable Coxregression model was built to evaluate the impact of each variable on progression to muscle-invasive disease. We developed a Cox-based nomogram for the prediction of patientspecific probability of disease progression at 2 and 5 years after TURB. Decision curve analysis (DCA) was performed to assess the clinical benefit with the use of our nomogram. Results: Overall, 636 patients met the inclusion criteria and were retained for the analyses. Within a median follow up of 92 months (IQR 56-118), disease progression occurred in 91 (14\%) patients. On multivariable analysis, age, early recurrence $(<12$ months) and tumor size $\geq 3 \mathrm{~cm}$ were found to be independent predictors of progression. The Harrel C-index of our model was 0.75 . DCA showed superior net benefits for the nomogram within a threshold probability of progression between 5\% and $25 \%$ compared to the strategies of treating all/none. Limitations are inherent to the retrospective design. Conclusion: We provided a risk-stratification tool that helps identify the individual risk of disease progression for patients with intermediate risk NMIBC. This tool outperforms standard strategies in the threshold probability range of interest and could help in the selection of the optimal intravesical therapy regimen based on the individual risk of disease progression.

\author{
88 \\ IMPACT OF SECOND LOOK TRANS- \\ URETHRAL RESECTION OF THE BLADDER \\ ON ONCOLOGICAL OUTCOMES OF COMPLETED \\ RESECTED T1HG NON-MUSCLE INVASIVE \\ BLADDER CANCER PATIENTS
}

Francesco Soria ${ }^{1}$, David D' Andrea ${ }^{2}$, Marco Moschini ${ }^{3}$, Paolo Gontero $^{1}$ and Shahrokh Shariat ${ }^{2}$

${ }^{1}$ Division of Urology, Azienda Ospedaliera Città della Salute e della Scienza di Torino, University

of Turin, Turin, Italy;

${ }^{2}$ Department of Urology and Comprehensive Cancer

Center, Medical University of Vienna,

Vienna General Hospital, Vienna, Austria;

${ }^{3}$ Department of Urology, Urological Research Institute,

Vita-Salute University, San Raffaele

Scientific Institute, Milan, Italy

Background/Aim: The second look transurethral resection of the bladder (TURB) has become an essential component of the management of $\mathrm{T} 1$ bladder cancer as it improves staging (i.e. understaging) and helps eradicate the tumor, thereby improving response to intravesical Bacillus Calmette-Guerin (BCG) therapy. However, recent data suggest that not all patients benefit from a second intervention with associated risks. Indeed, the value of a second look TURB has been put into question, especially in patients with a complete first resection. The aim of our study was to evaluate the impact of second look TURB on the oncological outcomes of patients with T1HG non-muscle invasive bladder cancer (NMIBC) treated with BCG. Patients and Methods: We performed a multicenter retrospective analysis of completely resected (complete resection and detrusor muscle present in pathologic specimen) T1HG NMIBC patients subsequently treated with adjuvant intravesical BCG who underwent (group A) or not (group B) second look TURB. The impact of second look TURB on survival outcomes was evaluated with the Kaplan-Meier method and differences were compared with Log-rank test. Multivariable survival analyses were performed with Cox's proportional hazard regression method. Results: Overall, 248 patients were included for the analyses. Of these, 205 (83\%) underwent second look TURB before BCG treatment, while $43(17 \%)$ did not. Second look TURB pathology was T0, Ta and T1 in 78\%, $11 \%$ and $11 \%$ of patients, respectively. On univariable analyses, second look TURB was significantly associated with better recurrence-free survival $(p=0.04)$, but not with progressionsfree, cystectomy-free, cancer-specific and overall survival (all $p>0.5$ ). On multivariable analyses that adjusted for the effects of standard clinico-pathologic characteristics, second look TURB was not associated with any of oncologic outcomes. Limitations are inherent to the retrospective design, possible selection bias, and sample size. Conclusion: In this retrospective study, second-look TURB did not convey oncologic advantages in completely resected T1HG patients treated with adjuvant BCG. Awaiting results from prospective trials, these data suggest that second look TURB could be avoided in a highly selected group of patients. 
89

\section{PROGNOSTIC VALUE OF THE SYSTEMIC INFLAMMATION MODIFIED GLASGOW PROGNOSTIC SCORE IN PATIENTS WITH UPPER TRACT UROTHELIAL CARCINOMA (UTUC) TREATED WITH RADICAL NEPHROURETERECTOMY: RESULTS FROM A LARGE MULTICENTER INTERNATIONAL COLLABORATION}

Francesco Soria ${ }^{1}$, David D'andrea ${ }^{2}$, Jose Karam ${ }^{3}$, Christopher Wood ${ }^{3}$, Morgan Rouprêt ${ }^{4}$, Vitaly Margulis ${ }^{5}$, Pierre Karakiewicz ${ }^{6}$, Alberto Briganti ${ }^{7}$, Jay Raman ${ }^{8}$, Karim Bensalah ${ }^{9}$, Yair Lotan ${ }^{5}$, Paolo Gontero ${ }^{1}$, Mesut Remzi ${ }^{10}$, Kilian Gust ${ }^{10}$ and Shahrokh Shariat ${ }^{10}$

${ }^{1}$ Division of Urology, Azienda Ospedaliera Città della Salute e della Scienza di Torino, University of Turin, Turin, Italy;

${ }^{2}$ Department of Urology, Medical University of Vienna, Vienna, Austria;

${ }^{3}$ Department of Urology, The University of Texas MD Anderson Cancer Center, Houston, TX, U.S.A.; ${ }^{4}$ Department of Urology, Sorbonne Université, GRC N ${ }^{\circ}$, ONCOTYPE-URO, AP-HP, Hôpital Pitié-Salpêtrière, Paris, France;

${ }^{5}$ Department of Urology, University of Texas Southwestern Medical Center, Dallas, TX, U.S.A.; ${ }^{6}$ Department of Urology, University of Montreal, Montreal, Canada;

${ }^{7}$ Department of Urology, Urological Research Institute, Vita-Salute University, San Raffaele Scientific Institute, Milan, Italy; ${ }^{8}$ Division of Urology, Penn State Milton S.Hershey Medical Center, Hershey, PA, U.S.A.; ${ }^{9}$ Department of Urology, University of Rennes, Rennes, France;

${ }^{10}$ Department of Urology, Medical

University of Vienna, Vienna, Austria

Background/Aim: Pre-treatment measures of systemic inflammatory response have been shown to act as prognosticators in several cancers and, therefore, have been combined in a variety of scores. The modified Glasgow prognostic score (mGPS), resulting from the combination of preoperative levels of C-reactive protein and albumin, has been investigated as predictor of survival outcomes in genitourinary malignancies, including prostate, kidney and bladder cancers. However, the role of mGPS in upper tract urothelial carcinoma (UTUC) remains poorly investigated. The aim of our study was to evaluate the prognostic role of mGPS for the prediction of oncologic outcomes in a retrospective large multicenter cohort of UTUC patients treated with radical nephroureterectomy (RNU). Patients and
Methods: We retrospectively analyzed a multicenter cohort of patients treated with RNU for clinically non-metastatic UTUC. Kaplan-Meier curves were built to compare recurrence-free (RFS), cancer-specific (CSS) and overall survival (OS) rates between groups. Multivariable logistic regression analyses were performed to evaluate the ability of mGPS to predict non-organ confined (NOC) disease and lymph-node involvement at RNU. Multivariable Coxregression models were performed to evaluate the prognostic effect of mGPS on survival outcomes. Decision curve analysis (DCA) was used to assess the clinical impact on decision making of mGPS. Results: Overall, 2,492 patients were included in the study. Of these, 849 (34\%), 1,489 (60\%) and $154(6 \%)$ had a mGPS of 0,1 and 2, respectively. mGPS was associated with characteristics of tumor aggressiveness such as tumor stage, grade, lymph-nodal status, presence of lympho-vascular invasion (LVI) and necrosis in pathologic specimen. On multivariable logistic regression analyses, mGPS predicted lymph-node involvement and NOC at RNU (both $p<0.05$ ). On univariable and multivariable Coxregression analyses that adjusted for the effect of standard prognosticators, higher mGPS was independently associated with RFS, CSS, and OS (all $p<0.001$ ). In DCA, inclusion of mGPS did not improve the net benefit of the model for the prediction of oncologic outcomes compared to standard prognosticators. Conclusion: mGPS was independently associated with clinicopathologic features and survival outcomes after RNU. However, despite this association, it had no impact on clinical decision making as it did retain its independent, clinical value when adjusted for standard prognosticators. Future studies should investigate the role of mGPS in a panel of preoperative markers for the prediction of pathologic features in UTUC patients, thus possibly improving the selection for neoadjuvant therapy, especially in the age of immunotherapy and lymphadenectomy.

\section{0}

\section{PREDICTIVE FACTORS FOR THE ABSENCE OF RESIDUAL DISEASE AT REPEATED TURBT}

Francesco Soria ${ }^{1}$, David D'Andrea ${ }^{2}$, Marco Moschini ${ }^{3}$, Rodolfo Hurle ${ }^{4}$, Renzo Colombo ${ }^{3}$, Vincenzo Altieri ${ }^{5}$, Kilian Gust ${ }^{2}$, Alberto Briganti ${ }^{3}$, Shahrokh Shariat ${ }^{2}$ and Paolo Gontero ${ }^{1}$

${ }^{1}$ Division of Urology, Azienda Ospedaliera Città della Salute e della Scienza di Torino, University of Turin, Turin, Italy;

${ }^{2}$ Department of Urology, Medical University of Vienna, Vienna, Austria;

${ }^{3}$ Department of Urology, Urological Research Institute, Vita-Salute University, San Raffaele

Scientific Institute, Milan, Italy; 
${ }^{4}$ Department of Urology, Istituto Clinico Humanitas Istituto di Ricovero e Cura a Carattere ScientificoClinical and Research Hospital, Milan, Italy;

${ }^{5}$ Department of Urology, University of Salerno, Salerno, Italy

Aim: The aim of the study was to identify predictors of pT0 on repeated transurethral resection of the bladder (re-TURBT) in patients with pT1 high-grade (HG) tumors. Patients and Methods: This multicenter retrospective study included 291 patients with pT1HG non-muscle invasive bladder cancer (NMIBC) from four different centers. Only patients who underwent a complete TURBT were included in the study. ReTURBT was defined as a second resection which involved the site of the first TURBT. A multivariable logistic-regression model was performed to evaluate the predictors of pT0 at reTURBT. A nomogram was built to calculate the probability of obtaining a negative histology at re-TURBT. A decision curve analysis was performed to evaluate the performance of the nomogram and to assess its net benefit. Results: Overall, 291 patients with pT1HG bladder cancer (BCa) were included in the study. Of these, $43(15 \%)$ harbored recurrent tumors. Detrusor muscle in the pathologic specimen at TURBT was present in 193 patients (70\%). En-bloc resection was performed in 12 patients while photodynamic diagnosis (PDD) was used in 18 patients. On multivariable logistic regression analysis, detrusor muscle in specimen $(\mathrm{HR}=2.14, p=0.01)$, concomitant carcinoma in situ (CIS) (HR=0.23, $p=0.002)$ and resection performed en-bloc (HR=9.68, $p=0.04)$ were independent predictors of pT0 at re-TURBT. Decision curve analysis showed the net benefit for the nomogram based on the multivariable model for each probability over 0.3 compared to the strategy to perform a re-TURBT in all pT1HG tumors. Conclusion: The presence of detrusor muscle in TURBT specimen, the absence of concomitant CIS and the en-bloc resection were able to predict a negative histology at re-TURB, opening the door over the possibility to avoid it in well-selected patients. External validation and prospective studies are urgently needed to confirm our hypothesis generating finding and to establish an algorithm for optimal management of $\mathrm{T} 1$ bladder cancer.

\section{1}

\section{SIURO PRIAS ITA WORKING GROUP: 9-YEAR EXPERIENCE OF ACTIVE SURVEILLANCE}

Fabio Badenchini ${ }^{1}$, Fabiana Zollo ${ }^{1}$, Vincenzo Altieri ${ }^{2}$, Umberto Barbaresi $^{3}$, Michele Battaglia ${ }^{4}$, Bollito Enrico ${ }^{5}$, Marco Borghesi ${ }^{3}$, Giacomo Cicchetti ${ }^{6}$,

Piergiuseppe Colombo ${ }^{7}$, Maurizio Colecchia ${ }^{8}$, Luigi Da Pozzo ${ }^{9}$, Pasquale Ditonno ${ }^{4}$, Marco Fabiano ${ }^{2}$, Michelangelo Fiorentino ${ }^{10}$, Jacopo Frizzi ${ }^{11}$, Ezio Frego ${ }^{12}$,
Michele Gallucci ${ }^{13}$, Gianluca Grimaldi ${ }^{4}$, Giorgio Guazzoni ${ }^{14}$, Rodolfo Hurle ${ }^{14}$, Alberto Lapini ${ }^{11}$, Francesco Maiorino $^{2}$, Francesco Minisola ${ }^{13}$, Rodolfo Montironi ${ }^{15}$, Giorgio Napodano ${ }^{16}$, Aurelio Paganelli ${ }^{17}$, Biagio Paolini ${ }^{8}$, Luisa Pasini ${ }^{14}$, Carlo Patriarca ${ }^{18}$, Alessandro Pili ${ }^{11}$, Antonio Ranieri ${ }^{17}$, Maria Rosa Raspollini ${ }^{19}$, Marco Roscigno ${ }^{9}$, Roberto Sanseverino ${ }^{16}$, Steno Sentinelli ${ }^{20}$, Marco Tanello ${ }^{12}$, Andrea Turci ${ }^{6}$, Tiziana Rancati ${ }^{1}$, Tiziana Magnani ${ }^{1}$, Henk Luiting ${ }^{21}$, Giario Conti ${ }^{22}$ and Riccardo Valdagni ${ }^{23}$

${ }^{1}$ Prostate Cancer Program, Fondazione IRCCS, Istituto Nazionale dei Tumori, Milan, Italy; ${ }^{2}$ Division of Urology, Azienda Ospedaliera Universitaria Ruggi, Salerno, Italy;

${ }^{3}$ Division of Urology, Policlinico S. Orsola

Malpighi, Bologna, Italy;

${ }^{4}$ Division of Urology, Policlinico, Bari, Italy;

${ }^{5}$ Division of Pathology, Ospedale

S. Luigi Gonzaga, Orbassano, Italy;

${ }^{6}$ Division of Urology, Ospedale M. Bufalini, Cesena, Italy; ${ }^{7}$ Division of Pathology, Istituto Clinico Humanitas, IRCCS, Rozzano, Milan, Itlay;

${ }^{8}$ Division of Genito-Urinary Pathology, Fondazione

IRCCS, Istituto Nazionale dei Tumori, Milan, Italy;

${ }^{9}$ Division of Urology, Ospedale Giovanni

XXIII, Bergamo, Italy;

${ }^{10}$ Division of Pathology, Policlinico

Sant'Orsola Malpighi, Bologna, Italy;

${ }^{11}$ Division of Urology, Azienda Ospedaliera

Universitaria Carreggi, Florence, Italy;

${ }^{12}$ Division of Urology, Ospedale Civile, Desenzano, Italy;

${ }^{13}$ Division of Urology, Istituto Regina Elena, Rome, Italy;

${ }^{14}$ Division of Urology, Istituto Clinico

Humanitas, IRCCS, Rozzano, Milan, Italy;

${ }^{15}$ Division of Pathology, Ospedali

Riuniti Torrette, Ancona, Italy;

${ }^{16}$ Division of Urology, Ospedale

Umberto I, Nocera Inferiore, Italy

${ }^{17}$ Division of Urology, ASST Bergamo Est, Bergamo, Italy;

${ }^{18}$ Division of Pathology, Ospedale Sant'Anna, Como, Italy;

${ }^{19}$ Division of Pathology, Azienda Ospedaliera Università

Carreggi, Firenze, Italy;

${ }^{20}$ Division of Pathology, Istituto Regina Elena, Rome, Italy;

${ }^{21}$ Department of Urology, Erasmus University

Medical Centre, Rotterdam, Netherlands;

${ }^{22}$ Division of Urology, Ospedale Sant'Anna, Como, Italy;

${ }^{23}$ Radiation Oncology, Università di Milano, Fondazione IRCCS, Istituto Nazionale dei Tumori, Milan, Italy

Background/Aim: Active Surveillance (AS) is proposed for patients with low-risk localized prostate cancer with the aim 
to reduce overtreatment by radical treatment strategies (surgery, external radiotherapy and brachytherapy). This abstract reports the 9-year experience of the SIUrO Prostate cancer Research International: Active Surveillance (PRIAS) ITA working group, composed of 13 Italian centers participating in the PRIAS protocol. Possible predictors of reclassification during AS were also evaluated. Matherials and Methods: In December 2009 SIUrO-PRIAS-ITA working group started including patients in the PRIAS protocol. Eligibility criteria are: prostate-specific antigen at diagnosis
(iPSA) $\leq 10 \mathrm{ng} / \mathrm{ml}$, Gleason Score (GPS) $\leq 6$, clinical stage $\leq \mathrm{T} 2 \mathrm{a}$, PSA density $\leq 0.2 \mathrm{ng} / \mathrm{ml} / \mathrm{cc}$, maximum of 2 positive cores at diagnostic biopsy. In case of saturation biopsy, up to $15 \%$ of the total number of cores can be positive. No limit of positive cores is required if mpMRI results negative or GPS $3+3$ is confirmed at fusion biopsy. During AS PSA examinations are performed every 3 months and visits every 6 months in the first 2 years, while PSA is performed every 6 months and visits yearly thereafter. Repeated biopsy is scheduled at years 1, 4, 7 and 10, and subsequently every 5
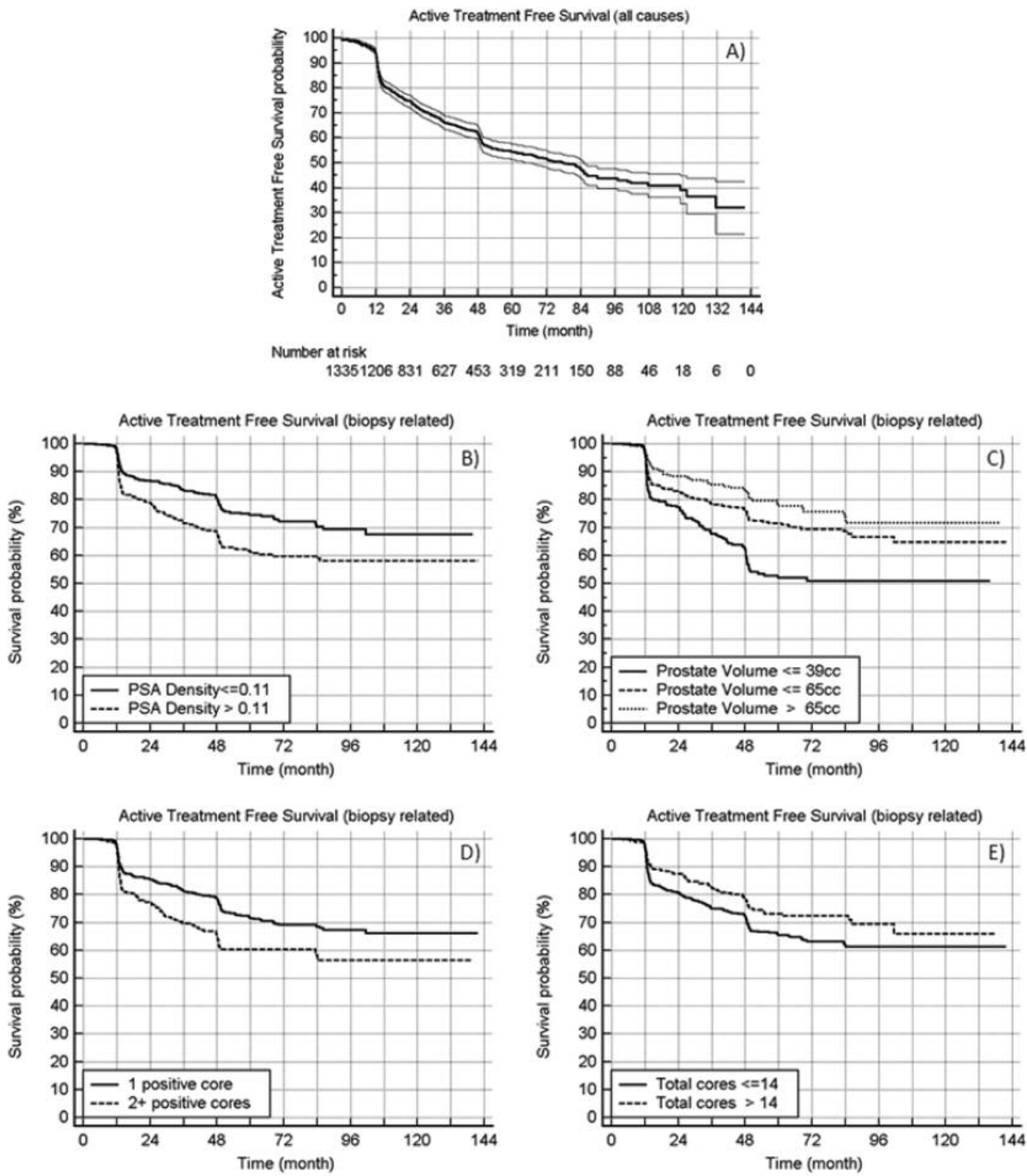

Figure 1. Kaplan-Meier curves for active treatment free survival: (A) overall survival; (B) as a function of PSA density; (C) as a function of prostate volume; $(D)$ as a function of number of positive cores at diagnostic biopsy; $(E)$ as a function of number of total cores at diagnostic biopsy. 
years. Patients drop out from AS due to upgrading (GPS $>6$ ) or upsizing ( $>2$ positive cores, before the introduction of MRI criteria in 2013) at re-biopsy. Active Treatment Free Survival (ATFS) was evaluated using Kaplan-Meier analysis. ATFS probability was reported at 48 and 84 months for significative variables. The relationship between ATFS and clinical risk factors at diagnosis was evaluated through the log rank test and Cox proportional hazard model. Results: Between December 2009 and October 2018, 1335 prostate cancer patients were included in PRIAS. Median age at inclusion was 66 years $($ range $=42-81)$, median iPSA $5.9 \mathrm{ng} / \mathrm{ml}($ range $=0.2-$ 10). Of the patients, $450(34 \%)$ had at least two positive cores at diagnostic biopsy, while 1261 (95\%) were classified as T1c at digital rectal exploration (DRE). Median time on AS was 31 months (range=1-138). Of the 543 patients $(41 \%)$ that dropped out from AS, 329 were due to upgrading and/or upsizing at re-biopsy (190/329 at first re-biopsy, one year after inclusion), 44 patients were lost to follow-up, 70 chose to end active surveillance and switched to active treatment or Watchful Waiting, 11 dropped out due to non PCa related death, 24 patients switched to Watchful Waiting due to age and 65 patients discontinued AS due to other reasons (i.e. clinical worsening, radiological progression, anxiety, PSA doubling time). Biopsy-driven ATFS resulted to be correlated to PSA density $\leq 0.11 \mathrm{ng} / \mathrm{ml} / \mathrm{cc}(p<0.0001$, ATFS at 48 months $79 \%$ $v s .67 \%$, ATFS at 84 months $69 \%$ vs. $59 \%$ ), prostate volume $(p<0.0001$, volume stratified in three groups: $\leq 39 \mathrm{cc}, 40-65$ cc, $>65 \mathrm{cc}$, ATFS at 48 months $60 \%$ vs. $75 \%$ vs. $83 \%$, ATFS at 84 month $49 \% v s .66 \% v s .69 \%$, respectively), number of positive cores at diagnostic biopsy $(p<0.0001$, ATFS at 48 months $78 \%$ vs. $63 \%$, ATFS at 84 month $67 \%$ vs. $53 \%, 1$ core vs. $2+$ cores, respectively), and number of total cores at diagnostic biopsy $(p=0.0068$, ATFS at 48 months $71 \%$ vs. $78 \%$, ATFS at 84 month $59 \%$ vs. $71 \%, \leq 14 v s .>14$ cores, respectively). Kaplan Meier curves are shown in Figure 1. Best fit multivariable Cox model for biopsy-driven ATFS resulted in a 3-variable model (overall $p<0.0001$ ) including age (continuous variable, risk factor, $\mathrm{HR}=1.0187$ ), prostate volume (continuous variable, protective factor, $H R=0.9824$ ) and number of positive cores at diagnosis (continuous variable, risk factor, $H R=1.2561)$. Conclusion: Most of drop out events occurred after the re-biopsy at 12 months, which should probably be considered as a confirmatory biopsy. PSA density, prostate volume, number of positive cores and total cores at diagnostic biopsy were correlated with biopsy-related ATFS. Cox multivariable model suggested age at diagnostic biopsy, number of positive cores according to literature (1) and prostatic volume as predictive variables of ATFS. The protective result of prostate volume could be explained with an incorrect sampling in high-volume prostate.

1 Bokhorst LP, Valdagni R, Rannikko A, Kakehi Y, Pickles T, Bangma $\mathrm{CH}$ and Roobol MJ: A decade of active surveillance in the PRIAS Study: An update and evaluation of the criteria used to recommend a switch to active treatment. Eur Urol 70(6): 954-960, 2016. PMID: 27329565. DOI: 10.1016/j.eururo.2016.06.007

\section{2 \\ TAKING CHARGE OF PATIENTS ON ACTIVE SURVEILLANCE: THE ROLE OF THE PSYCHOLOGIST DURING "SAPI" (THE FIRST ACCESS VISIT)}

Letizia De Luca ${ }^{1}$, Fabio Badenchini ${ }^{1}$, Paola Dordoni ${ }^{1}$, Teresa Di Florio ${ }^{1}$, Tiziana Magnani ${ }^{1}$, Cristina Marenghi ${ }^{1}$, Nicola Nicolai ${ }^{1}$, Fabiana Zollo ${ }^{1}$, Riccardo Valdagni ${ }^{2}$ and Lara Bellardita ${ }^{1}$

${ }^{1}$ Prostate Cancer Porogram, Fondazione IRCCS Istituto Nazionale dei Tumori, Milan, Italy; ${ }^{2}$ Department of Oncology and Hemato-oncology, Fondazione IRCCS - Istituto Nazionale dei Tumori, Milan, Italy

Background/Aim: Over the past two decades, as a consequence of the increase of prostate cancer diagnoses, active surveillance (AS) has become an acceptable suitable option for men with low- and very low-risk clinically localized disease (1). This strategy is available for those who are willing to accept an observational program and at the same time the risk of disease reclassification or progression to be spared the well-known complications associated with surgery and radiation, so as to preserve quality of life longer (2). The choice of being included in AS protocols represents a very important transition in patients' life because it marks the beginning of an engagement process in relation to their disease, which involves activation in a series of essential steps able to keep the cancer and its possible evolution under monitoring. AS patients, in fact, are closely monitored through PSA blood tests every three months, follow-up visits every 6 months and periodic prostate biopsies (the first after 12 months from diagnosis, the other according to protocol). Therefore, deciding to start an AS program means, full-fledged, living with the tumor, condition that might be expected to evoke psychological distress, particularly in the initial phase, during which patients begin to come in touch with what "being on AS" means. Given the complexity of such a choice and the importance of getting familiar with the protocol, our Institution provides for men who decide to enter the AS program a structured first visit (called SAPI - AS first encounter), that consists of a clinical examination aimed to offer all the necessary information. This abstract aims to present the SAPI format as conceived by the Prostate Cancer Unit of Fondazione IRCCS Istituto Nazionale dei Tumori (Milan, Italy), with a particular focus on the role of the psychologist in a similar context. Materials and Methods: Our 
Institution provides a structured first visit aimed to give patients all the information they need to start their AS program. Taking into consideration the benefits of a multidisciplinary approach, starting from 2018, the SAPI format includes the presence of a clinical psychologist, in addition to the presence of clinicians. The clinical psychologist aims to conduct an initial assessment of the patients' psychological condition. Currently, SAPI is structured as follows: before the clinical visit with the oncologist, psychologist intercepts the patient and asks him to fill in two questionnaires: MAX-PC (to assess anxiety) and Mini-MAC (to assess ways of coping with cancer), to be returned filled in before the visit. While the patient is in the room with the doctor, the psychologist elaborates the two questionnaires live, in order to outline a personal profile with respect to the two variables of interest, considered fundamental both from the literature and the clinical practice. Once the interview with the doctor is concluded, the patient meets the psychologist in a dedicated room where a real psychological interview is conducted to evaluate patients' general psychological health. Results: The starting point of this psychological session is represented by an accurate presentation of the outcomes of the questionnaires, giving the patient an overview of his anxiety level and coping strategies in relation to the tumor. However, the clinical practice emphasizes how giving back the patient information regarding these two variables prompts the possibility of touching many other subjects, strictly interconnected with their management of the pathology. In fact, the encounter with our AS patients during the post-SAPI psychological interview allowed us to identify 6 recurring thematic areas: 1. "decompression" (this moment seems to represent a very important opportunity for patients to put in order the information received during the clinical visit, supported by a health professional involved in their care path); 2. "from the diagnosis up to now" (another important and usual theme is represented by the need to recall - often for the first time - what happened from the time of diagnosis until today, going through the decision making process, with a particular reference to the emotions experienced throughout the whole route); 3. "the sphere of intimacy" (doubts or fears related to the sphere of sexual intimacy often emerge during the post-SAPI interview, as if the patients, now well informed from a physical point of view, would take a space to elaborate the emotions linked to having to face a so symbolically full pathology); 4. "cancer and couple" (patients are often accompanied by their spouse and post-SAPI clinical interview represents a useful occasion to face the disease from both perspectives, giving the couple the opportunity - often for the first time - to confront each other with mutual fears and anxieties in a safe environment); 5. "cancer and family" (this is also a very precious occasion to begin to face up to family issues related to cancer, for example coping strategies of other family members or system's reorganization resulting from the disease); 6. "thinking about my life" (this moment often represents an opportunity to reflect on existential themes regarding the past or present sphere of life, also not directly related to the disease but of great importance for the patients). If the psychologist evaluates the presence of a psychological discomfort above threshold, the case is reported to the rest of the clinical team. If during AS experience patients deal with the only possible side effect of AS, anxiety, everyone will have the possibility of being taken in charge by our Institution psychologists. Conclusion: This 10-month experience with this new format gave us the opportunity to underline the importance of such a multidisciplinary approach even with those who have already decided to take part in AS program, to assess the initial level of compliance and indicate the presence of a structure able to take care of their patients at 360 degrees.

\section{3 \\ ACUTE AND LATE TOXICITY OF HYPOFRACTIONATED RADIOTHERAPY FOR LOCALIZED PROSTATE CANCER: IMRT VS. HELICAL TOMOTHERAPY}

\author{
Annaisabel Rese, Francesco Pastore, Diego Toledo, \\ Ferdinando Francomacaro, Gianluca Ametrano, \\ Fabrizio Cammarota and Vincenzo Iorio
}

Radiation Oncology, EMICENTER, Casavatore, Italy Aim: To evaluate the incidence of acute and late toxicity after hypofractionated radiotherapy using linac intensitymodulated radiotherapy (IMRT) compared with helical tomotherapy. Materials and Methods: From September 2016 to March 2018, 110 consecutive patients with localized prostate cancer [cT1-2, Gleason score $(\mathrm{GS})<8$, prostatespecific antigen (PSA) $<10 \mathrm{ng} / \mathrm{ml}$ ) were randomized to linac IMRT and to helical tomotherapy (TOMO). Fifty-five patients were treated with linac IMRT and 55 with TOMO. Patients were monitored before therapy, weekly during therapy, 2 weeks, 3 and 6 months after radiotherapy was completed, using the Radiation Therapy Oncology Group (RTOG) gastrointestinal (GI) and genitourinary (GU) toxicity grading scale. Patients received radiotherapy schedule according to histology reports following international guidelines. Doses were prescribed to planning target volumes (PTVs) as the followings: $72 \mathrm{~Gy}(2.4 \mathrm{~Gy} / \mathrm{fx})$ to PTV-whole prostate and $64.5 \mathrm{~Gy}(2.15 \mathrm{~Gy} / \mathrm{fx})$ to PTV-prostate, and seminal vescicles in 30 fractions with simultaneous integrated boost (SIB) technique. Doses to abdominal cavity, both femoral heads, bladder, and rectum were constrained below each tissue tolerance. Results: Median age of the patients was 72.5 years (range $=55-86$ ). At the end of the treatment (6 weeks), 16/55 (29\%) patients in the TOMO group vs. 19/55 (34.5\%) patients in the linac IMRT group had G1-G2 grade of GI toxicity ( $p=0.009)$, while $2 / 55(4 \%)$ 
patients in the TOMO group vs. $4 / 55(7.3 \%)$ patients in the linac IMRT group had G3 grade of GI toxicity. Twenty-seven of $55(49 \%)$ patients in the TOMO group $v s .31 / 55(56 \%)$ patients in the linac IMRT group had G1-G2 grade of GU toxicity $(p=0.04)$, while $1 / 55(1.8 \%)$ patients in the TOMO group vs. $3 / 55(5.5 \%)$ patients in the linac IMRT group had G3 grade of GU toxicity. No G4 grade of GI and GU toxicity was showed. After 6 months from the end of the treatment, no patients in the TOMO group vs. $2 / 55$ (3.6\%) patients in the linac IMRT group had G1-G2 grade of GI toxicity, while $1 / 55(1.8 \%)$ patients in the TOMO group vs. 2/55 (3.6\%) patients in the linac IMRT group had G3 grade of GU toxicity. Conclusion: Acute toxicity was very low, while most of the recorded symptoms decrease over time in both groups. A small increase in mild toxicity, statistically significant, was observed in the linac IMRT group when compared with TOMO group. Our study confirmed that tomotherapy allows for safe moderate hypofractionation, offering a shorter overall treatment time, a lower rate of acute and late toxicities and providing potentially more economic health care.

\section{4 \\ THE ROLE OF THE CLINICAL TRIAL RESEARCH NURSE IN THE MANAGEMENT OF ACTIVE SURVEILLANCE PROTOCOLS}

$\underline{\text { Teresa Di Florio }}^{1}$, Tiziana Magnani ${ }^{1}$, Cristina Marenghi ${ }^{1}$, Fabio Badenchini ${ }^{1}$, Fabiana Zollo ${ }^{1}$, Paola Dordoni ${ }^{1}$, Letizia De Luca ${ }^{1}$, Elisa Campi ${ }^{2}$, Valentina Doldi ${ }^{2}$, Barbara Avuzzi ${ }^{3}$, Barbara Noris Chiorda ${ }^{3}$, Sara Morlino ${ }^{3}$, Sergio Villa ${ }^{3}$, Antonella Messina ${ }^{4}$, Alessandra Casale ${ }^{4}$, Rodolfo Lanocita ${ }^{4}$, Mario Catanzaro ${ }^{5}$, Antonio Tesone ${ }^{5}$, Tullio Torelli ${ }^{5}$, Silvia Stagni ${ }^{5}$, Alberto Macchi ${ }^{5}$,

Roberto Salvioni ${ }^{5}$, Nicola Nicolai ${ }^{5}$,

Nadia Zaffaroni ${ }^{2}$ and Riccardo Valdagni ${ }^{1,6}$

${ }^{1}$ Prostate Cancer Unit, ${ }^{2}$ Molecular Pharmacology Unit, ${ }^{3}$ Division of Radiation Oncology 1, ${ }^{4}$ Division of Radiology, ${ }^{5}$ Division of Urology, ${ }^{6}$ Department of Oncology and Hemato-oncology, Fondazione IRCCS, Istituto Nazionale dei Tumori, Milan, Italy

Background/Aim: Active surveillance (AS) is an observational approach that can be proposed in alternative to radical treatments (surgery, external radiotherapy and brachytherapy) for patients with low and very low risk prostate cancer and a long-life expectancy (longer than 10 years) with the aim to avoid treatment side effects. Patients who choose AS adhere to a protocol-based follow-up scheme with periodic follow-up visits, PSA tests, biopsies and multiparametric magnetic resonance imaging (mpMRI), with specific discontinuation criteria. This abstract examines the role of the Clinical Trial Research Nurse in the management of patients on AS protocols. Materials and Methods: The Clinical Trial Research Nurse participates in the weekly inclusion visits held Wednesdays from 9:00-12:00 am. The nurse helps the medical oncologist in collecting the documents and the general information, signing the informed consent form, and filling the case report form (CRF). Once all the information on AS is delivered to the patient, the nurse proposes the participation in the Tissue Bank side protocol Bioteca. If the patient accepts, the informed consent is signed, while blood and urine samples are collected. The biological material is processed and stored to be used in further translational studies (e.g. biomarkers related to prostate cancer aggressiveness). Both blood and urine were collected at the enrollment; instead, in the follow-up period (every 12 months), only blood was collected once before the 12-month biopsy and then one sample per year. The Clinical Trial Research Nurse collects the biological material, labels the test-tubes with the patient identification number, hands them to the laboratory, updates the database and plans the next follow-up collection, generally in concomitance with the follow-up visit date (before the rectal exploration) or the biopsy (before the procedure). During AS, patients undergo mpMRI at critical check-up points. These radiological images are used to direct the following examinations, i.e. biopsy administration and biopsy technique (random vs. targeted). Another task of the Clinical Trial Research Nurse is to evaluate the mpMRI of patients on AS on a weekly basis. If no lesions or Prostate Imaging Reporting and Data System (PI-RADS) score $<2$ lesions are reported, no targeted biopsy is required and the patient continues AS according to standard examinations. If PI-RADS score $\geq 3$ lesions are described, the case is submitted to the PCU Core Team experts meeting, that includes urologists, radiation oncologists, medical oncologists and the dedicated radiologists, in order to determine the need for a fusion biopsy. Since 2015, the Clinical Trial Research Nurse is taking part to the weekly Tumor Boards. The nurse schedules the cases to be discussed and prepares the summary of the case, by specifying the diagnostic question and including the anamnesis. During the Tumor Board she prioritizes cases and takes note of the decisions made by the clinicians, and finally her task is to inform the patient about the decision taken. The case discussions are then recorded in a dedicated file, in order to keep track of it. The Clinical Trial Nurse is also in charge of scheduling patients for fusion biopsy. She calls the patient to communicate the examination date and collects informations on his medications, and she sends the informed consent and the preparation form. Finally, the nurse informs urologists and radiologists about the biopsy schedule. Results: Since 2014, a Clinical Trial Research Nurse has joined the Prostate Cancer Unit. Up to 2018, a total of 1036 patients were enrolled in the AS at National 
Cancer Institute, 505 patients were enrolled after the introduction of Clinical Trial Nurse, and 474/1036 are still on AS. Considering the BIOTECA study, 231 patients were enrolled; 22 patients $(9.5 \%)$ dropped-out from the study, generally because of upsizing or upgrading of the biopsy. Since 2015, a total of $398 \mathrm{mpMRI}$ and 217 fusion biopsies have been performed with up to 6 mpMRI per week and 10 fusion biopsies per month. About 10 patients per week are usually planned and worked-up to be evaluated by the Tumor Board in clinical meeting. Since 2015, a total of 914 patients have been discussed by a multidisciplinary team, mainly for PSA doubling time, enrollement in AS or Watchful Waiting evaluation of mpMRI or other stadiation exams. Conclusion: The role of the Clinical Trial Research Nurse as an active figure of the multidisciplinary management of prostate cancer patients is effective and efficient in supporting the inclusion of patients in clinical trials, improving patient's compliance and adherence to protocols, facilitating the synergy among the specialists of the multidisciplinary team. By participating in the multidisciplinary activities of the Prostate Cancer Unit the Clinical Trial Research Nurse has gained experience of interdisciplinary interaction and coordinated management of prostate cancer patients.

We thank Fondazione I. Monzino for the support to the activities of the Prostate Cancer Program at Fondazione IRCCS Istituto Nazionale dei Tumori, Milan, Italy.

\section{6}

\section{LONG-TERM ONCOLOGICAL OUTCOMES} IN POST-SURGERY LOCOREGIONAL RECURRENT PCA TREATED BY 3D-CONFORMAL OR VOLUMETRIC
MODULATED-ARC RADIOTHERAPY

\author{
Claudia Sorce $^{1}$, Letizia Ferella1, Francesca Vittorini ${ }^{1}$, \\ Emilia Varrassi ${ }^{1}$, Pietro Franzese ${ }^{1}$, Mario Di Staso ${ }^{1}$, \\ Valeria Ruggieri ${ }^{1}$, Giovanna Riccitelli ${ }^{1}$, Anna Aleggiani ${ }^{1}$, \\ Teresa Grausberg ${ }^{1}$, Francesco Marampon ${ }^{2}$, Paolo Tini ${ }^{3}$, \\ Agnieszka Chalaszczyk ${ }^{1}$, Gianmarco Grimaldi ${ }^{1}$, \\ Ernesto Di Cesare ${ }^{4}$, Vincenzo Tombolini ${ }^{5}$, \\ Carlo Masciocchi ${ }^{4}$ and Giovanni Luca Gravina ${ }^{1}$ \\ ${ }^{1}$ Division of Radiotherapy, University \\ of L'Aquila, L'Aquila, Italy; \\ ${ }^{2}$ Department of Oncological and Pathological Radiological \\ Sciences, University of Rome, Rome, Italy; \\ ${ }^{3}$ Division of Radiotherapy, University \\ of Siena, Sienna, Itlay; \\ ${ }^{4}$ Division of Radiology, University \\ of L'Aquila, L'Aquila, Italy; \\ ${ }^{5}$ Division of Radiotherapy, La Sapienza \\ University, Rome, Italy
}

Aim: The present retrospective analysis aimed to report the fractionation schedules and the long-term oncological outcomes of men with post-surgery non-metastatic macroscopic locoregional relapsed prostate cancer (Pca) and treated by $3 \mathrm{D}$ conformal radiotherapy (3DCRT) or volumetric intensity-modulated radiotherapy (VMAT). Methods: Fortyfour patients with post-surgery non-metastatic radiologically or histologically confirmed macroscopic locoregional relapsed Pca were retrospectively reviewed. Inclusion criteria were a post-surgery biochemical relapse with positron emission tomography (PET) positive and/or computed tomography (CT) scan positive findings in the prostatic fossa and/or in the locoregional lymph nodes. The treatment was performed with VMAT or 3DCRT and a wide range of fractionations were used according to the target dimension or the site of disease. Target delineation was performed according to the Radiation Therapy Oncology Group (RTOG) atlas for salvage Pca. Daily patient set up was implemented by image-guided RT (IGRT) approach using cone-beam CT (CRCT) images before every treatment session. Progressionfree survival (PFS), defined as biochemical and/or radiological progression, and overall survival (OS) were used as main oncological measures. Results: The mean age of studied population was 68.8 years $(95 \% \mathrm{CI}=67.2-70.5)$ and the mean pre-RT prostate-specific antigen (PSA) was $2.1 \mathrm{ng} / \mathrm{ml}$ (95\% CI=1.4-4.7). Thirty-two (72.7\%) men received antiandrogen therapy before RT. Ten men (22.7\%) were treated with VMAT and $25(56.8 \%)$ also received IGRT. Macroscopically relapsed tumour was in prostate fossa and in the regional nodes in $39(88.6 \%)$ and $5(11.6 \%)$ patients, respectively. Eight $(18.2 \%)$ men received the irradiation of the only prostate fossa (70-76 Gy in 35-37 fractions) and 3 patients $(7 \%)$ received irradiation of the only macroscopic relapsed Pca within prostate fossa (36.25 Gy in 5 fractions). Twenty-six $(59.1 \%)$ men received the irradiation of the prostate fossa (70-76 Gy in 35-37 fractions) with elective irradiation of regional nodes (45-50.4 Gy in 25-28 fractions). Two men $(4.5 \%)$ received SIB-VMAT delivered to prostate fossa (68.4 Gy in 38 fractions) and macroscopically relapsed Pca (76 Gy in 38 fractions) and 3 men (7\%) received SIBVMAT delivered to elective locoregional lymph nodes (49.551 Gy in 30-33 fractions) and PET+ nodes (60-66 Gy in 3033 fractions). Finally, $2(4.5 \%)$ patients received VMAT delivered to PET+ lymph nodes (40 Gy in 5 fractions). The post-RT PSA nadir $(0.082 \mathrm{ng} / \mathrm{ml} ; 95 \% \mathrm{CI}=0-016)$ was achieved at a mean time of 11 months. The actuarial PFS and OS were $123.8(95 \% \mathrm{CI}=103.97-143.7)$ and 138 months (95\% CI $=121-154.4)$ with the rate of men free from progression and alive of $79.5 \%$ and $91 \%$. Six men progressed in prostate fossa and 3 developed bone lesions. The mean PSA at the last follow-up was $3.75 \mathrm{ng} / \mathrm{ml}$ (95\% CI=0-10.1). Conclusion: Approximately 15-30\% of men with localized Pca will experience biochemical recurrence (BCR) after 
radical prostatectomy and a significant proportion of them will experience radiologically documented relapse. In men with non-metastatic disease, high performance status and limited locoregional tumour burden RT may reduce the risk of local progression and may maintain a curative intent. Literature data seems to suggest that this radiation treatment is associated with non-trivial risks of acute and long-term toxicity. Thus, RT of non-metastatic men with macroscopic locoregional relapsed Pca poses a unique challenge for radiotherapists owing to the age-related limited life expectancy and the high treatment effectiveness of multimodality treatment strategies. Tailored age-related defined treatment strategies should be identified to improve the quality of life of this population.

\section{7 \\ CONVENTIONAL HIGH-DOSE VS. HYPOFRACTIONATED-RADIOTHERAPY IN PROSTATE CANCER: A SYSTEMATIC-REVIEW OF RCTS WITH TRIAL SEQUENTIAL ANALYSIS}

\author{
Letizia Ferella $^{1}$, Francesca Vittorini ${ }^{2}$, Claudia Sorce $^{3}$, \\ Agnieszka Chalaszczyk ${ }^{3}$, Gianmarco Grimaldi ${ }^{3}$, \\ Pietro Franzese ${ }^{3}$, Valeria Ruggieri ${ }^{3}$, Anna Aleggiani ${ }^{3}$, \\ Teresa Grausberg ${ }^{3}$, Giovanna Riccitelli ${ }^{3}$, Emilia Varrassi $^{3}$, \\ Mario Di Staso ${ }^{3}$, Carlo Masciocchi ${ }^{4}$ and \\ Giovanni Luca Gravina ${ }^{3}$ \\ ${ }^{1}$ Division of Radiotherapy, Fondazione IRCCS \\ Istituto Nazionale Dei Tumori, Milan, Italy; \\ ${ }^{2}$ Division of Medical Physics, University \\ of L'Aquila, L'Aquila, Italy; \\ ${ }^{3}$ Division of Radiotherapy, Univerity \\ of L'Aquila, L'Aquila, Italy; \\ ${ }^{4}$ Division of Radiology, University \\ of L'Aquila, L'Aquila, Italy
}

Aim: The present systematic review aimed to comparatively assess the oncological outcomes and the late toxicities of randomized control trials (RCTs) testing moderate hypofractionated radiotherapy (RT) and conventional highdose RT. A grounded body of evidence suggests that for patients with low-risk cancer cumulative doses of 75.6 to 79.2 Gy in conventional fractionation can be used, while for intermediate and high risk localized prostate cancer (PCa) doses up to $81 \mathrm{~Gy}$ are warranted to improve tumor control. Materials and Methods: The study inclusion criteria were RCTs involving patients with localized PCa and comparing moderately hypofractionated regimens (HRT) (2.4-4 Gy daily fractions for 15-30 treatments) with conventional radiotherapy CRT (1.8-2 Gy daily fractions for 40-45 treatments) with curative intent. Dose-escalated external beam
RT (EBRT) in conventional regimens was defined as a total dose of $76 \mathrm{~Gy}$ or greater delivered in $2 \mathrm{~Gy}$ per fraction. A minimum follow-up period of 5 years was required for the oncological outcomes. Studies were eligible irrespective of whether image guidance was used to deliver the intervention or if intensity-modulated RT (IMRT) or 3DCRT treatments were delivered. On the other hand, studies were excluded if essential information was missing that could not be obtained from the authors. The evaluated endpoints were cancerspecific survival (CSS), overall survival (OS), biochemicalfree survival (BFS) and late Grade 2 or greater genitourinary (GU) and gastrointestinal (GI) toxicity. BFS was defined according to the original trials. As further endpoint, the statistical reliability of cumulative data assessed in the systematic review was defined using the trial sequential analysis (TSA). This power statistical approach helps researchers in concluding if the evidence drawn by a specific metanalysis can be considered statistically definitive or other trials and additional recruitment have to be performed. Results: A total of 2,501 men were enrolled in the 4 RCTs included in this systematic review. One-hundred fifty-four men received HRT and 1,247 received CRT, respectively, with no significant difference between the two arms $(p=0.87)$. Overall, the vast majority of the study cohorts was composed of men with intermediate $(63.7 \% ; 1,594 / 2,501)$ or high-risk $(34.3 \% ; 857 / 2,501) \mathrm{PCa}$. The percentage of men receiving androgen deprivation therapy (ADT) was $37.4 \%(935 / 2,501)$ and the $\mathrm{T}$ stage ranged from $\mathrm{T} 1$ to $\mathrm{T} 4$. All Gleason scores were included and the pre-RT PSA was below $100 \mathrm{ng} / \mathrm{ml}$. Four different hypofractionated regimens were used with dose per fraction ranging from 3 to $3.4 \mathrm{~Gy}$. When the oncological performances of RT were analyzed, the rate of men dying from tumor-related events at the last follow-up was $3.03 \%$ (38 events) in the HFRT group and $4.25 \%$ (53 events) in the CRT group. HFRT regimens did not perform significantly better than dose-escalated conventional regimens in terms of CSS [relative risk $(\mathrm{RR})=1.011 ; 95 \% \mathrm{CI}=0.985-1.035$; $p=0.413]$. The rate of men dying from any events at the last follow-up was $16.43 \%$ (177 events) in the HFRT group and $17.97 \%$ (190 events) in the CRT group. Even when the OS was used as the oncological endpoint, HFRT regimens did not perform significantly better than dose-escalated conventional regimens $(\mathrm{RR}=1.008 ; 95 \% \mathrm{CI}=0.976-1.049 ; p=0.637)$. When the late toxicity was analyzed in terms of GU and GI rate of G2 or greater toxicity, HFRT was not more toxic than doseescalated CRT (2,194 randomized patients). Late G2 or greater GI toxicity occurred in 14.1\% (155 events) and in $15.5 \%$ (169 events) of men treated with HFRT and doseescalated CRT, respectively. When the late toxicity was compared in terms of RR, HFRT regimens did not perform significantly worse than dose-escalated conventional regimens $(\mathrm{RR}=0.892 ; 95 \% \mathrm{CI}=0.593-1.343 ; p=0.231)$. The TSA we report supported the acceptance that HFRT is an 
alternative to dose-escalated CRT in the treatment of $\mathrm{PCa}$ patients when OS, CCS and BFS were used as outcomes. These findings were clear observing the TSA curves trend since the cumulative $\mathrm{Z}$-curves crossed the trial sequential monitoring boundaries, but did not cross either alphaspending boundaries or the no-difference lines (the futility boundaries). All these findings supported the conclusiveness, from a statistical point of view, of the non-inferiority of HFRT respect to CRT regimens. Conclusion: The treatment of localized PCa with definitive radiotherapy has been one of the longest-duration treatment regimens employed by the radiotherapists. With these regimens, radiation therapy can be inconvenient for patients, costly, as well as a resourceintensive endeavor for radiation departments. Concern for patient safety is one reason for the long treatment. Recent technologic advances such as IMRT and image-guided RT have dramatically improved the accuracy of radiation delivery and decreased cumulative radiation dose to organs at risk surrounding the prostate. These technical advancements have allowed exploration of the safety and the efficacy of hypofractionated fractionations. Moderately hypofractionated radiation treatments are an effective alternative to high dose conventional regimens both in terms of long-term oncological outcomes and toxicity. However, the more effective fractionation still remains an open questions and head to head RCTs are needed.

\section{8}

\section{UTILITY OF SERUM MARKERS IN THE ASSESSMENT OF PERIOPERATIVE AND POSTOPERATIVE MORBIDITY AND MORTALITY AFTER RADICAL CYSTECTOMY FOR MUSCLE INVASIVE BLADDER CANCER}

\author{
Francesco Claps, Matteo Boltri, Francesca Migliozzi, \\ Giacomo Rebez, Nicola Pavan, Michele Rizzo, \\ Giovanni Liguori and Carlo Trombetta \\ Urology Clinic, Department of Medicine, Surgery and \\ Health Science, University of Trieste, Trieste, Italy
}

Background/Aim: Despite significant improvements in surgical techniques, radical cystectomy (RC) remains a highly morbid operation. The ability to predict complications and create prevention strategies is crucial for the surgical decision-making process and for optimizing treatment outcomes. Recently, there is growing interest in the association of preoparetive inflammation and immunonutritional serum markers with post-surgical complications and survival outcomes. The aim of this study was to investigate and compare the ability of preoperative Controlling Nutritional Status (CONUT), Prognostic Nutritional Index (PNI), neutrophil to lymphocyte ratio
(NLR), platlet to lymphocyte ratio (PLR), lymphocyte to monocyte ratio (LMR), systemic immune-inflammation index (SII), albumin, fibrinogen and PCR to predict perioperative and postoperative morbidity and mortality after RC. Materials and Methods: We retrospectively evaluated 164 patients who underwent open RC for muscle-invasive bladder cancer (MIBC) at our Institute between December 2004 and June 2018. We excluded those patients who received neoadjuvant therapy and patients in whom data were incomplete. Covariates were analyzed to determine associations with complication rates (according to the Clavien-Dindo system), mean hospitalization length, 30-days readmission rates and 90-days mortality. A multivariable binomial logistic regression determined associations with post-surgical outcomes taking into account age, sex, urinary diversion, $\mathrm{pT}$ stage and each serum marker, or American Society of Anesthesiologists (ASA) classification and Charlson Comorbity Index (CCI) categorization. Results: Cut-off values to discriminate threshold of these biomarkers were determined calculating the ROC curve and the maximum Youden index. The mean age of patients at surgery was 72.1 years (range $=46-88$ ) and the majority of urinary diversions were ileal conduit (78.1\%). Overall, 44 (26.8\%) patients experienced a major complication (Clavien grade $\geq 3$ ) and there were $9(5.5 \%)$ deaths within 3 months of surgery. ASA, CONUT, NLR, PLR, SII and PCR showed statistically significant differences in distribution of complications (all $p<0.05)$. There were no differences in mean hospitalization length while CONUT, PNI, fibrinogen, PCR, SII and CCI were statistically associated with 30-days readmission. Fibrinogen was the only serum marker associated with 90days mortality $(p=0.01)$. Multivariable binomial logistic regression analysys confirmed the association of CONUT, SII, ASA, NLR, PCR and fibrinogen with surgical complications (all $p<0.05$ ). Conclusion: Preoperative inflammation and immuno-nutritional serum markers based on standard laboratory measurements may be simple and inexpensive potentially effective risk-assessment tools to predict outcomes after RC. Further investigations should be necessary to confirm these results.

\section{9 \\ TREND AND CHARACTERISTICS OF INCIDENTAL RENAL MASSES DISCOVERED AT TIME OF AUTOPSY}

Francesco Claps $^{1}$, Venus Shafiei ${ }^{2}$, Carmelo Morreale ${ }^{1}$, Matteo Boltri ${ }^{1}$, Francesca Migliozzi ${ }^{1}$, Lorena Di Marco ${ }^{1}$, Nicola Pavan ${ }^{1}$, Michele Rizzo ${ }^{1}$, Giovanni Liguori ${ }^{1}$, Rossana Bussani ${ }^{2}$ and Carlo Trombetta ${ }^{1}$

${ }^{1}$ Urology Clinic, Department of Medicine, Surgery and Health Sciences, University of Trieste, Trieste, Italy; 
${ }^{2}$ Institute of Pathological Anatomy and Histology, Department of Medicine, Surgery and Health

Sciences, University of Trieste, Trieste, Italy

Background/Aim: Doubtless, advances in imaging technology such as abdominal ultrasounds, computed tomography (CT) and magnetic resonance imaging (MRI) are playing an important role in the diagnosis of renal masses (RM) and renal cell carcinoma (RCC) before the presence of clinical symtomps. This aspect has prompted interest in active surveillance (AS) as a treatment option for the small masses, especially if supported by an imaging-guided biopsy that can characterize the tumor that most likely will not contribute to cancer specific mortality. To the best of our knowledge, contemporary data evaluating the temporal trend of pathological characteristics of the incidental RM and their relationship with cancer-related death are not available. The aim of this study was to analyze incidence trend, changes in clinical characteristics, pathological features, and cancer-related death of patients with RM incidentally discovered at time of autopsy in a long period of time. Materials and Methods: Data were retrieved from the autopsy register of the Pathology Department of a single tertiary referral center from 15,086 consecutive autopsies performed between January 2004 and December 2017. Patients with previous history of primary RCC and patient with a kidney metastatic involvement from other tumors were excluded from this study. Results: Overall, $184(1.22 \%) \mathrm{RM}$ were found. Benign and malignant lesions were $32(17.4 \%)$ and $152(82.6 \%)$, respectively. The mean age at death was 82 years $(\mathrm{SD}=11.2)$, while the majority of patients were female $(56.5 \%)$. Histologically, RMs were oncocytoma $13(7.1 \%)$, angiomyolipoma $13(7.1 \%)$, papillary adenoma 4 (2.2\%), cystic nephroma $3(1.6 \%)$, metanephric tumor 2 (1.1\%), clear-cell RCC $136(73.9 \%)$ in which occured $2(1.5 \%)$ sarcomatoid variant, papillary RCC $2(1.1 \%)$, chromophobe RCC $5(2.7 \%)$, carcinoma of the collecting ducts of Bellini 5 (2.7\%), nephroblastoma $1(0.5 \%)$. Considering the malignancies, pathological stage was: pT1 $126(80.3 \%)$, pT2 $10(6.4 \%)$, pT3 $16(10.2 \%)$ and pT4 $5(3.2 \%)$; in $16(10.2 \%)$ cases, malignancy was the cause of death. Temporal trend of incidence of RM and RCC decreased significantly over the years ( $p=0.01$ and $p=0.01$ respectively). While the average age at death, sex and the distribution of the different histotypes remained constant over the time, RM found in the last years were increasingly smaller ( $p=0.04)$ and only in one case in the last seven years RM was the cause of the patient's death. Conclusion: The autopsy finding of incidental RM is decreasing. Although the distribution of the different kidney tumor histotypes appears constant, the mean size of the lesions that are incidentally identified at autopsy are increasingly smaller and more harmless. The incidental finding of RM is nowadays more common using the modern imaging techinques.
101

PATHOLOGICAL FINDINGS AT RADICAL PROSTATECTOMY OF BIOPSY NAÏVE MEN SUBMITTED TO MRI-TARGETED BIOPSY ALONE WITHOUT STANDARD SYSTEMATIC SAMPLING

\author{
$\underline{\text { Stefano Luzzago }}^{1}$, Michele Catellani ${ }^{1}$, \\ Francesco Alessandro Mistretta ${ }^{1}$, Andrea Conti ${ }^{1}$, \\ Ettore Di Trapani ${ }^{1}$, Antonio Brescia ${ }^{1}$, Paola Pricolo ${ }^{2}$, \\ Sarah Alessi ${ }^{2}$, Fabrizio Verweij ${ }^{1}$, Matteo Ferro ${ }^{1}$, \\ Deliu-victor Matei ${ }^{1}$, Giuseppe Renne ${ }^{3}$, Giuseppe Petralia ${ }^{2}$, \\ Gennaro Musi ${ }^{1}$ and Ottavio De Cobelli ${ }^{1}$
}

${ }^{1}$ Department of Urology, IEO European

Institute of Oncology, Milan, Italy;

${ }^{2}$ Department of Radiology, IEO, European

Institute of Oncology, Milan, Italy;

${ }^{3}$ Department of Pathology, IEO, European

Institute of Oncology, Milan, Italy

Background/Aim: Two prospective randomized trials have endorsed the use of multiparametric magnetic resonance imaging (mpMRI) as a triage test for prostate cancer (PCa) diagnosis in biopsy-naïve men. Moreover, the PRECISION trial assessed the superiority of targeted biopsy strategy (without standard cores) over systematic sampling for the detection of clinically significant (cs) PCa. We evaluated pathological findings at prostatectomy (RP) of biopsy naïve men submitted to MRI-targeted biopsy alone. Materials and Methods: We respected ethical guidelines of the Declaration of Helsinki. From 2016 to 2018, 138 biopsy naïve men with $\mathrm{PCa}$ suspicion, such as rising prostatespecific antigen (PSA), positive digital rectal examination (DRE), and a positive mpMRI (PI-RADS $\geq 3$ ) were submitted to in-bore MRI-targeted biopsies (2-5 cores per area) of lesions suggestive for $\mathrm{PCa}$, without standard sampling. Overall, 63 patients were then submitted to RP. Logistic regression analyses tested predictors of discordance between bioptic and pathological Grade Group (GG). Cancer location and tumor volume (TV) in the whole specimen were assessed. Finally, we evaluated how many csPCa $(\mathrm{GG} \geq 3)$ were underestimated (not seen at biopsy) and overestimated (not confirmed at RP) by performing only MRI-targeted biopsy. Results: Overall, median age, PSA and PSA density at RP were 63 (IQR=57-69), 6.7 $(\mathrm{IQR}=4.8-9.4)$ and 0.12 (IQR=0.09-0.2), respectively. Reported Index-Lesion (IL) PI-RADS score at in-bore biopsy was 3 in $13(20 \%), 4$ in $35(55.5 \%)$ and 5 in 15 $(24.5 \%)$ men, respectively. Median number of positive and overall cores performed were 2 (1-4) and 4 (3-5). Globally, $25(39.5 \%), 24(38 \%), 10(16 \%), 3(4.5 \%)$ and $1(2 \%)$ patients had a GG 1, 2, 3, 4 and 5 at biopsy, respectively. Median TV at RP was 5.5 (2.6-9) ml. Globally, there was a perfect overlap between IL at mpMRI and tumor location 
at $\mathrm{RP}$ in $54(86 \%)$ cases. In $4(44.5 \%)$ patients, there was a laterality mismatch (i.e. right $v$ s. left), while in $5(55.5 \%)$ men the lesion was on the same lobe but in a different region of the gland (i.e. apex vs. base). Overall, $19(30 \%)$, $16(25 \%), 4(6.5 \%)$ and $2(3 \%)$ men had csPCa, extracapsular extension (ECE), seminal vesicle invasion (SVI) and lymph nodes invasion (LNI) at RP, respectively. The discordance rate between bioptic and pathological GG was $44 \%$ (28/63). Of these patients, $5(18 \%)$ and $23(82 \%)$ had GG downgrading and upgrading, respectively. At logistic regression analysis, only max core length involvement ( $\mathrm{mm}$ of core with greatest involvement) [odds ratio $(\mathrm{OR})=1.2 ; 95 \% \mathrm{CI}=1.05-1.6]$ was associated with $\mathrm{GG}$ discordance. Finally, $3(5 \%)$ and $7(11 \%)$ csPCa were overestimated and underestimated by performing biopsies only in the target zone. At logistic regression analysis there were no predictors of $\mathrm{csPCa}$ under/overestimation. Conclusion: Roughly half of patients submitted to targeted biopsy strategy showed GG discordance between biopsy and RP specimens. Moreover, $11 \%$ of patients were affected by csPCa, which was misdiagnosed when standard biopsy cores were avoided. Future studies with larger cohorts of patients are needed to find predictors of csPCa underestimation.

\section{3}

\section{BLADDER FILLING PROTOCOL FOR RT PROSTATE: HOW FULL?}

Giovanna Mantello ${ }^{1}$, Jessica Andreatini ${ }^{1}$, Marco Valenti ${ }^{2}$, Sara Costantini ${ }^{1}$, Francesca Cucciarelli ${ }^{1}$, Maika Di Benedetto ${ }^{1}$, Letizia Fabbietti ${ }^{1}$, Francesco Fenu ${ }^{1}$, Andrea Maucieri ${ }^{1}$, Maria Montisci ${ }^{1}$, Lisa Vicenzi ${ }^{1}$, Andrea Benedetto Galosi ${ }^{3}$, Stefania Maggi ${ }^{2}$ and Massimo Cardinali ${ }^{1}$

${ }^{1}$ Department of Radiotherapy, Radiotherapy Department, AOU Ospedali Riuniti Ancona, Ancona, Italy; ${ }^{2}$ Department of Physics, Radiotherapy Department, AOU Ospedali Riuniti Ancona, Ancona, Italy; ${ }^{3}$ Department of Urology, Radiotherapy Department, AOU Ospedali Riuniti Ancona, Ancona, Italy

Aim: Bladder filling can determine displacements and deformations of prostate (1-3). For this reason, the patient must follow a preparation before each radiotherapy session. The purpose of this work was (i) to study patients who had records of their preparation, (ii) to analyze filling and maintenance of the bladder volume, (iii) to verify the preparation related to a better reproducibility, and (iv) to

\section{Trend of bladder volume during RT}
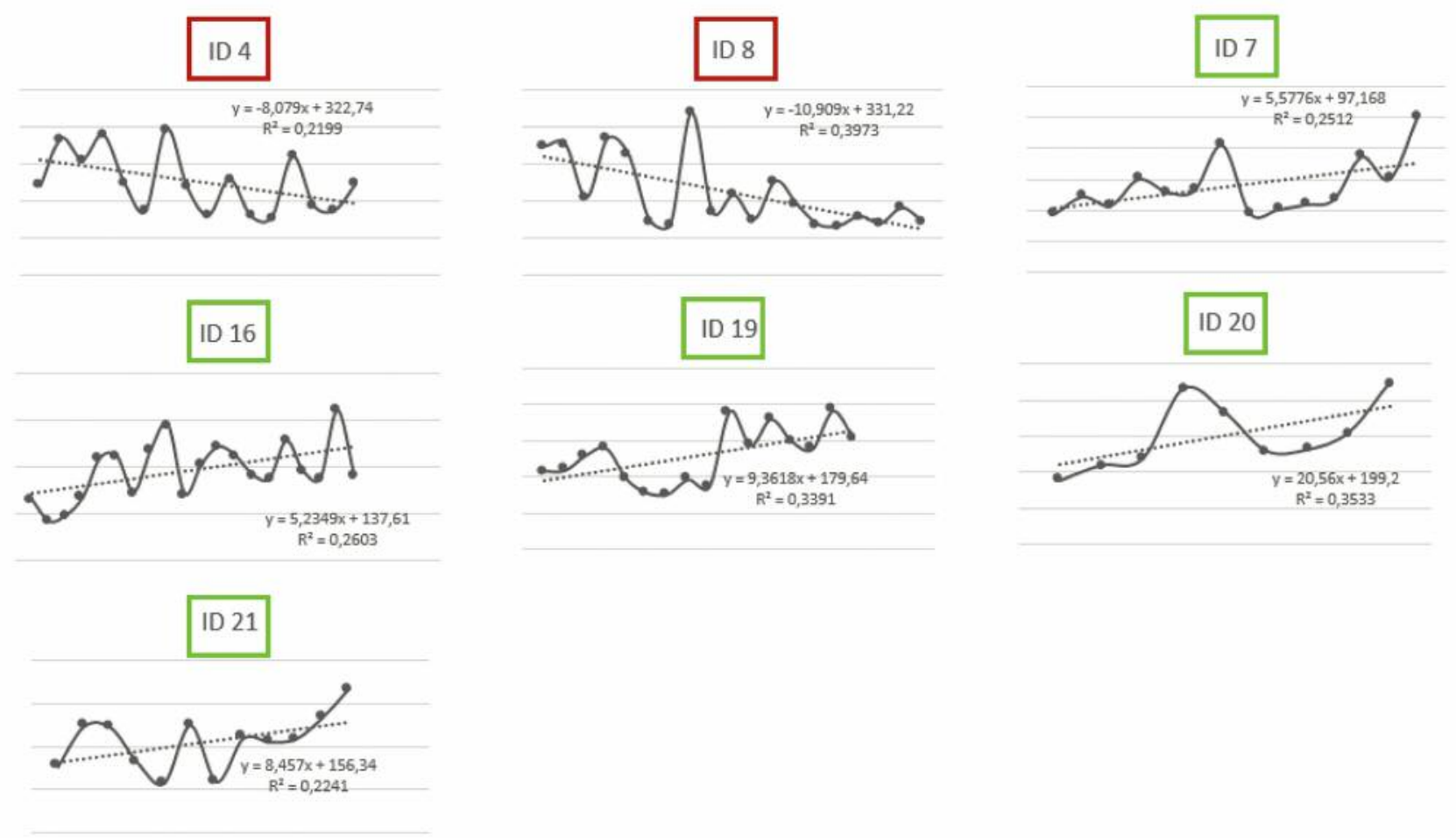

Figure 1. Trend of bladder volumes, increasing (5 cases) or decreasing (2 cases) during treatment. 


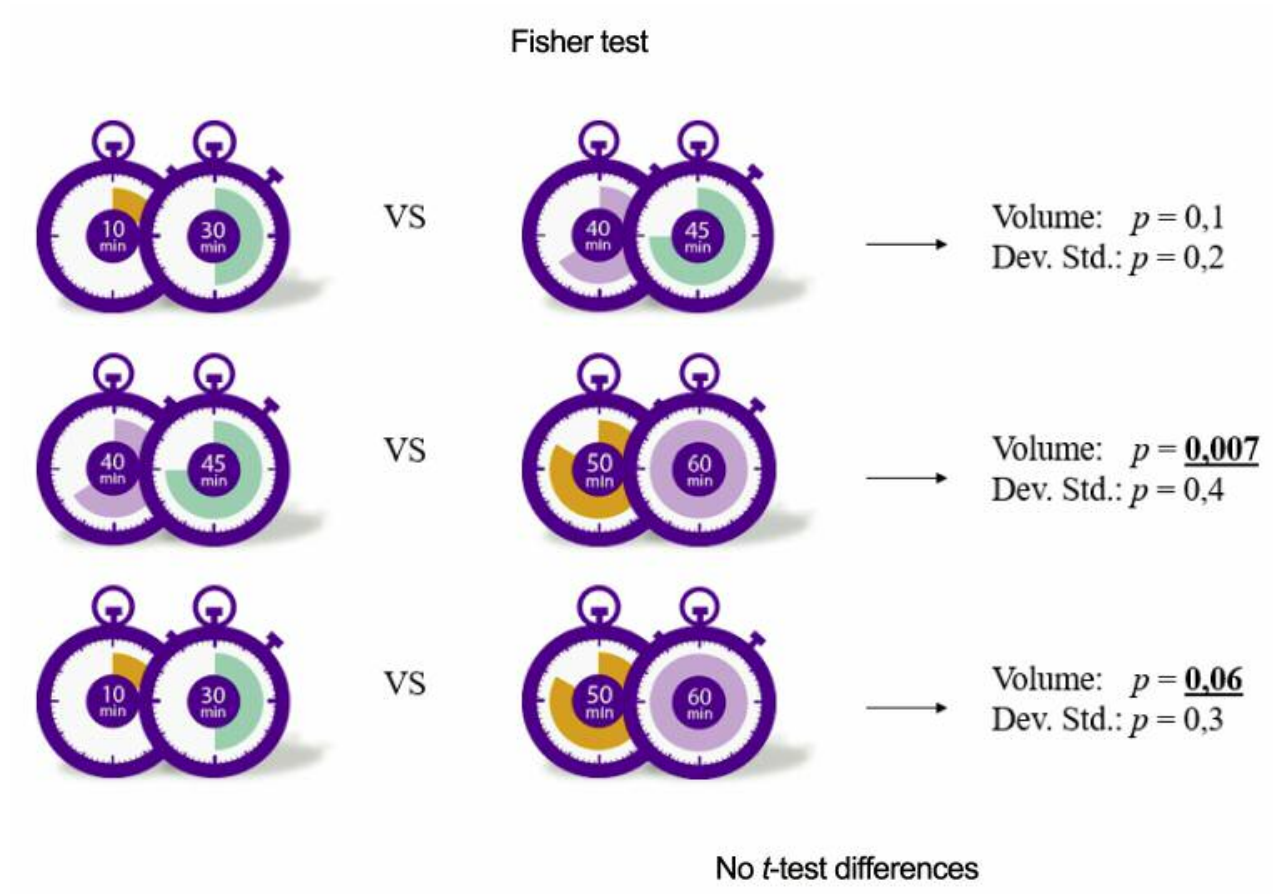

Figure 2. Fisher's and Student's t tests for volumes and for standard deviations (long preparations vs. short-medium preparation).

submit a dedicated protocol. Materials and Methods: A retrospective study was carried out on 26 patients with prostate cancer, who also had a recorded bladder preparation; the amount of water drank by the patient varied between $1 / 2$ liter and $3 / 4$ liter in a period of time between 10 and 60 min before computed tomography (CT) and treatment (Table I). For each patient the bladder was contoured both in the simulation CT (CT sim) and in all the cone-beam CTs (CBCTs) acquired during the treatment (excluding those where the total visualization of the bladder was not possible). The Eclipse treatment planning system (TPS) was used for contouring. The filling volumes and the maximum diameters were recorded in the 3 axes and the mean of the fillings, the standard deviation, the minimum and the maximum filling for each patient were calculated. In total, 423 bladders were contoured and examined. Results: According to the data collected, the patients were divided into seven groups according to preparations. None of the groups showed a better filling reproducibility. The average volume was $200 \pm 70 \mathrm{ml}$. Larger volume of bladders at the $\mathrm{CT}$ sim are not kept during treatment. Eleven from $26(42 \%)$ had a greater filling in the first session of treatment compared to the CT sim; on the contrary, 15/26 $(58 \%)$ presented a decreased bladder volume (up to $25 \%$ for 7 patients and over $50 \%$ for 8 patients). The trend of bladder volumes during the treatment showed no correlations in 19 cases, increasing trends in 5 cases and a decreasing trend in 2 cases (Figure 1). Further tests were
Table I. Amount of water drank by the patient in a period of time between 10 and $60 \mathrm{~min}$ before computed tomography $(\mathrm{CT})$ or treatment.

\begin{tabular}{lc}
\hline Number of patients per different preparation & Preparation \\
\hline 1 & $1 / 2$ lt in $10 \mathrm{~min}$ \\
8 & $1 / 2$ lt in $30 \mathrm{~min}$ \\
4 & $1 / 2$ lt in $40 \mathrm{~min}$ \\
4 & $1 / 2$ lt in $45 \mathrm{~min}$ \\
3 & $1 / 2$ lt in $50 \mathrm{~min}$ \\
4 & $1 / 2$ lt in $60 \mathrm{~min}$ \\
2 & $3 / 4 \mathrm{lt}$ in $40 \mathrm{~min}$ \\
\hline
\end{tabular}

carried out on three groups based on short (10-30 min), medium (40-45 $\mathrm{min}$ ) and long preparations (50-60 $\mathrm{min})$. On these, Fisher's and Student's t tests were performed, both for volumes and for standard deviations, coming to the conclusion that long preparations (50-60 min) lead to more empty bladders than short-medium ones. No differences were seen with the t-test (Figure 2). Conclusion: Average volumes of $200 \mathrm{ml}$ were recorded with a variability of 70 $\mathrm{ml}$. In most cases, the bladder volume decreased between the CT sim and the first session, even by over $50 \%$. Shortmedium preparations $(10 / 45 \mathrm{~min})$ led to larger volume than long preparations (50/60); none of the groups showed a better filling reproducibility; however, each preparation 
protocol leads to a stability of the bladder volume. As a result of our study, a standardized protocol with short preparation was proposed as follow: Refrain from urinating and from drinking, if not absolutely necessary, for $2 \mathrm{~h}$, then drink 0.51 of water in $5 \mathrm{~min}, 30 \mathrm{~min}$ before the CT sim or session. This protocol is introduced to the patient on the first visit; the patient is asked to train the bladder filling before CT sim.

1 Maggio A, Gabriele D, Garibaldi E, Bresciani S, Delmastro E, Di Dia A, Miranti A, Poli M, Varetto T, Stasi MP and Gabriele: Impact of a rectal and bladder preparation protocol on prostate cancer outcome in patients treated with external beam radiotherapy. Strahlenther Onkol 193: 722-732, 2017. PMID: 28620751. DOI: 10.1007/s00066-017-1163-4

$2 \mathrm{Lv}$ W, Yu D, He H and Liu Q: Monte Carlo simulations for dosimetry in prostate radiotherapy with different intravesical volumes and Planning Target Volume margins. PLoS ONE 11(7): e0159497, 2016. DOI: 10.1371/ journal.pone.0159497.

3 Doherty U, McNair H, Norman A, Miles E, Hooper S, Davies M, Lincoln N, Balyckyi J, Childs P, Dearnaley D and Huddart R: Variability of bladder filling in patients receiving radical radiotherapy to the prostate. Radiother Oncol 79(3): 335-340, 2006. PMID: 16781790. DOI: 10.1016/j.radonc.2006.05.007

\section{4 \\ USE OF THE INTRA-PROSTATIC CALCIFICATIONS FOR CORRECTION OF THE ORGAN MOTION}

$\underline{\text { Giovanna Mantello }}^{1}$, Giovanni Pierfederici ${ }^{1}$, Marco Valenti ${ }^{2}$, Sara Costantini ${ }^{1}$, Francesca Cucciarelli ${ }^{1}$, Maika Di Benedetto ${ }^{1}$, Letizia Fabbietti ${ }^{1}$, Francesco Fenu ${ }^{1}$, Andrea Maucieri ${ }^{1}$, Maria Montisci ${ }^{1}$, Lisa Vicenzi ${ }^{1}$, Andrea Benedetto Galosi ${ }^{3}$, Stefania Maggi ${ }^{2}$ and Massimo Cardinali ${ }^{1}$

${ }^{1}$ Radiotherapy Department, AOU Ospedali

Riuniti Ancona, Ancona, Italy;

${ }^{2}$ Department of Physics, AOU Ospedali

Riuniti Ancona, Ancona, Italy;

${ }^{3}$ Department of Urology, AOU Ospedali

Riuniti Ancona, Ancona, Italy

Aim: The treatment of the prostate alone can foresee the use of Fiducial gold markers (FMs) which are opportunely inserted by the urologist into the gland and act as a surrogate for the position of the prostate itself. The protocol of correction of the prostate position in these cases provides the search for the perfect overlap of the markers between simulation CT and imaging in bunkers. In patients suitable for the implant, the protocol is effective both in terms of local disease control and tolerance of healthy tissues. This method, while ensuring excellent results, does not adapt to every patient. As an alternative to the use of gold markers some authors have studied the use of natural markers such intra-prostatic calcifications (ICs), present in $90 \%$ of prostatectomies and visible in the images obtained in bunkers in at least $35 \%$ of the patients cone beam computed tomography (CBCT) $(1,2)$. On the contrary, periprostatic calcifications may undergo movements not related to those of the prostate and cannot serve as reliable indicators. The visibility of the ICs is dependent on the density and size of the calcifications and can have several advantages: they are natural, they do not create artifacts on images, and they cannot migrate because they are integrated into soft tissues. The aim of this work was to study the possibility of using the ICs, identified in the CT of centering and in CBCT, as natural markers to correct the organ motion inter fraction of the prostate during the entire course of radiotherapy. Materials and Methods: A total of 135 patients treated for prostate cancer were included. The study consisted of four steps: (i) selecting patients with intraprostatic calcifications on the CT sim images; (ii) checking their optimal visibility on the CBTC images; (iii) checking optimal match CBTCs/TC simulation images on the calcification; (iv) checking the adequacy of the $5 \mathrm{~mm}$ clinical target volume and planning target volume (CTV-PTV) margin (Figure 1). The $5 \mathrm{~mm}$ margin was chosen based on the experience gained in the last 12 years in the image-guided RT of the prostate with implantation of gold markers at our radiotherapy department. The study of the stability of the gold markers (3) and the calculation of the residual error after correction of the target position, led radiotherapists and physicists to consider a $5 \mathrm{~mm}$ CTV-PTV margin suitable to compensate for the uncertainty of positioning the target. Results: Out of 135 studied patients, 32 (23.7\%) showed calcifications, $20(14.8 \%)$ periprostatic and $12(8.9 \%)$ intraprostatic $87.5 \%$ of cases had clearly visible ICs on CBCT (147/168 images), $11.3 \%$ barely visible (19/168 images) and finally $1.2 \%$ scarcely visible/not visible (2 images) (Table I). In $89.2 \%$ of the images (106/118), the ICs allowed a good match and an optimal compensation of residual organ motion (OM) within $5 \mathrm{~mm}$. In $10.2 \%$ cases (12/118), gas in the rectum conditioned the correct use of ICs. The distension of the rectum in fact causes a shift in the posteroanterior direction and deformation of the prostate. Conclusion: The ICs when clearly visible $(>2 \mathrm{~mm})$ and are at least 3 , can be used as very reliable natural fiducial markers. This method potentially reduces the risks and costs associated with the implementation of conventional fiducial markers. Given the fairly high prevalence of these calcifications in the population of patients with prostate 


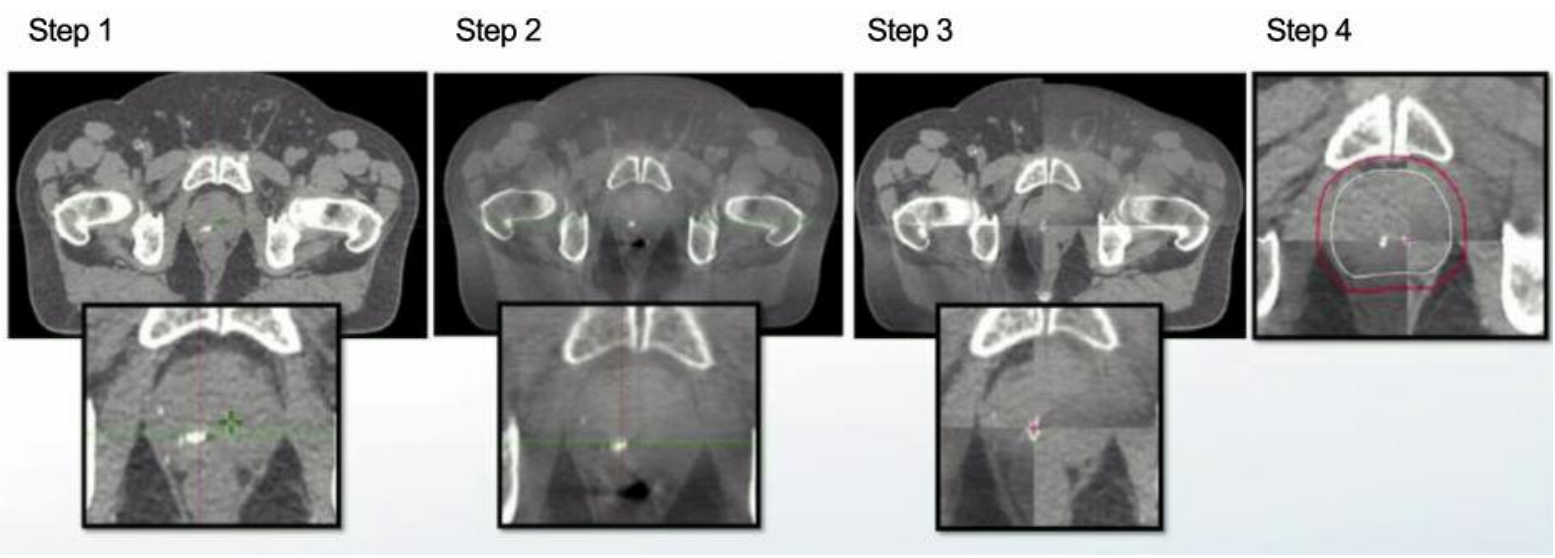

Figure 1. Main steps of the study. Step 1: Selecting patients with intraprostatic calcifications on the CT sim images; step 2: checking their optimal visibility on the CBTC images; step 3: checking optimal match CBTCs/CT sim images on the calcification; step 4: checking the adequacy of the $5 \mathrm{~mm} C T V$-PTV margin.

cancer, this non-invasive method could be more widely applied. To date, there are no indications on the margin to be adopted in a protocol that provides for the use of calcifications to compensate for the motion. However, some authors have suggested their use with margins of at least 6$10 \mathrm{~mm}$, wider than other IGRT strategies Indeed, the literature suggests $5-8 \mathrm{~mm}$ if co-registration is performed on soft tissues or implanted fiducials; $3-4 \mathrm{~mm}$ are foreseen only if a correction of the $\mathrm{OM}$ intrafraction with real-time tracking on fiducials markers is possible. In this study, we have shown, in agreement also with the studies reported in the literature, that in a high percentage of cases it is possible to make a good correction of the organ motion interfraction co-recording on the undertaking calcifications, if any, and that a $5 \mathrm{~mm}$ margin can compensate for the residual error after co-registration.

1 Stucchi P, Pozzi L, Bianchi C, Mordacchini C, Doino DP, Gottardo S, Antognoni $\mathrm{P}$ and Novario R: Intra- or periprostatic calcifications as natural fiducial markers for prostate localization in image-guided radiotherapy. Physica Medica: Eur J Med Phys 32(Suppl 1): 65, 2016. DOI: 10.1016/j.ejmp.2016.01.223

2 Sbai A, Thariatb J, Tachfoutid N, Pane Q and Lagrange JL: Calcifications intraprostatiques: Marqueurs fiduciels naturels dans la radiothérapie guidée par l'image du cancer de la prostate. Cancer - Radiothérapie 18: 740-744, 2014. DOI: $10.1016 /$ j.canrad.2014.07.161

3 Mantello G, Vagnoni B, Fenu F, Cucciarelli F, Costantini S, Scricciolo M, Vicenzi L, Valenti M, Maggi S and Cardinali M: Assessment of migration of intraprostatic fiducial markers during radiotherapy. Radiotherapy Oncology 115(Suppl 1): S819, 2015. DOI: https:// doi.org/ 10.1016/S0167-8140(15)41497-5
Table I. Visibility of intraprostatic calcifications on acquired cone beam computed tomography $(C B C T)$, per patient.

\begin{tabular}{lcccc}
\hline Patients & $\begin{array}{c}\text { Acquired } \\
\text { ID }\end{array}$ & \multicolumn{3}{c}{$\begin{array}{c}\text { Intraprostatic calcifications } \\
\text { visibility }\end{array}$} \\
\cline { 3 - 5 } & patient & $\begin{array}{c}\text { Clearly } \\
\text { visible }\end{array}$ & $\begin{array}{c}\text { Barely } \\
\text { visible }\end{array}$ & $\begin{array}{c}\text { Scarcely } \\
\text { visible }\end{array}$ \\
\hline 1 & 19 & $18 / 19$ & $1 / 19$ & - \\
2 & 18 & $17 / 18$ & $1 / 18$ & - \\
3 & 16 & $16 / 16$ & - & - \\
4 & 14 & $13 / 14$ & $1 / 14$ & - \\
5 & 16 & $16 / 16$ & - & - \\
6 & 16 & $15 / 16$ & $1 / 16$ & - \\
7 & 18 & $16 / 18$ & $2 / 18$ & - \\
8 & 10 & $7 / 10$ & $1 / 10$ & $2 / 10$ \\
9 & 11 & $9 / 11$ & $2 / 11$ & - \\
10 & 11 & $10 / 11$ & $1 / 11$ & - \\
11 & 11 & $10 / 11$ & $1 / 11$ & - \\
12 & 8 & - & $8 / 8$ & - \\
\hline
\end{tabular}

\section{5}

\section{RADIO-RECURRENT CASTRATION-RESISTANT PROSTATE CANCER: RESULTS OF A RECENT SERIES OF SALVAGE RADICAL PROSTATECTOMIES}

Giancarlo Marra $^{1}$, Giorgio Calleris ${ }^{1}$, Paolo Alessio ${ }^{1}$, Marco Oderda ${ }^{1}$, Francesca Pisano ${ }^{1}$, Antonino Battaglia ${ }^{1}$, Stefania Munegato ${ }^{1}$, Fernando Munoz ${ }^{2}$, Juan Palou ${ }^{3}$, Steven Joniau ${ }^{4}$, Salvatore Smelzo ${ }^{5}$, Thierry Piechaud ${ }^{5}$, Alessandro Morlacco ${ }^{6}$, Sharma Vidit ${ }^{6}$, Henk Van Der Poel ${ }^{7}$, Robert Jeffrey Karnes ${ }^{6}$ and Paolo Gontero ${ }^{1}$ 
${ }^{1}$ Department of Surgery, Urology, University of Turin, AOU Città della Salute e della Scienza, Turin, Italy; ${ }^{2}$ Department of Radiotherapy, Pasini Hospital, Aosta, Italy; ${ }^{3}$ Department of Urology, Fundaciò Puigvert,

Barcelona, Spain;

${ }^{4}$ Department of Urology, Leuven University

Hospitals, Leuven, Belgium;

${ }^{5}$ Department of Urology, Clinique Saint

Augustin, Bordeaux, France;

${ }^{6}$ Department of Urology, Mayo Clinic,

Rochester, MN, U.S.A.;

${ }^{7}$ Department of Urology, Netherlands Cancer

Institute, Amsterdam, the Netherlands

Aim: We investigated the oncological and functional outcomes of salvage radical prostatectomy (sRP) in the context of radio-recurrent Castration resistant prostate cancer (CRPC). Materials and Methods: We retrospectively analyzed data of 24 patients affected by radiotherapy (RT)-recurrent CRPC, who underwent $\mathrm{sRP}$ (open $\mathrm{n}=22$; robotic $\mathrm{n}=2$ ) at six tertiary referral centers from 2001 to 2014. We registered oncological, clinical and pathological features at sRP and during follow-up. Complications were graded using the Clavien-Dindo score. Continence was evaluated before sRP and at 6 and/or at 12 months, expressed as number of pads per day. Patients with insufficient data and/or with a followup shorter than 12 months were excluded. Results: Twentyfour men were included. At PCa diagnosis, original median prostate-specific antigen (PSA) was $18(\mathrm{IQR}=8.6-45) \mathrm{ng} / \mathrm{ml}$. At sRP, median age was 69 years $(\mathrm{IQR}=65-72)$ and median ECOG score $0(\mathrm{IQR}=0-0)$. Men underwent salvage surgery a median of $76(\mathrm{IQR}=48-107)$ months after primary treatment. Excluding two patients who were under androgen-deprivation therapy (ADT) at the time of sRP, median pre-sRP PSA was 7.4 (IQR=3.6-13.6). Considering medians, number of removed and positive nodes was $15.5(\mathrm{IQR}=4.5-23.2)$ and 0 $(\mathrm{IQR}=0-2.5)$, respectively; operating time was $220 \mathrm{~min}$ (IQR=189-277), median blood loss $500 \mathrm{ml}(\mathrm{IQR}=450-700)$ ( $\mathrm{n}=3$ needed post-operative transfusions). Median hospital stay was 7 (IQR=4-9) days. Major complications and overall complications were encountered by $40 \%$ and $73 \%$ of the patients, respectively. At final pathology, nearly half of the patients (47.6\%) had Gleason Score (GS) 9, 23\% had GS 8 and $28.5 \%$ had GS 7 ; the proportion of positive surgical margins and of non-organ-confined disease was noticeable (65\% and $64 \%$, respectively). First post-operative PSA was below $0.2 \mathrm{ng} / \mathrm{ml}$ in $37.5 \%(\mathrm{n}=9)$. After 6 and/or 1 -year follow-up, $33 \%$ of patients was continent, whilst $44 \%$ of men had severe incontinence ( $>2$ pads/day). At a median followup of 47.8 months (IQR=25-85): i) $17.4 \%$ were disease-free; ii) $82.6 \%$ had recurrence; iii) $33.3 \%(\mathrm{n}=8)$, and $8.3 \%(\mathrm{n}=2)$ died for cancer specific other causes, respectively. Secondline therapies were started by 4 patients a median of 47.8 months after sRP. Conclusion: sRP for CRPC is an extremely complex surgery, which entails high complication rates and comorbidities and poor curative rates. Nevertheless, the procedure may be curative or post-pone CRPC status relapse and/or initiation of second line therapies in a minority of patients. Larger series are needed to confirm these findings and to identify potential candidates with the greatest expected benefit.

\section{6 \\ ACUTE TOXICITY IN HYPOFRACTIONATED RADIOTHERAPY OF PROSTATE CANCER: VMAT VERSUS 3DCRT WITH DAILY IGRT}

Gianmarco Grimaldi ${ }^{1}$, Francesca Vittorini ${ }^{2}$, Emilia Varrassi $^{3}$, Pietro Franzese ${ }^{3}$, Valeria Ruggieri ${ }^{3}$, Mario Di Staso ${ }^{3}$, Francesco Marampon ${ }^{4}$, Letizia Ferella ${ }^{1}$, Claudia Sorce ${ }^{1}$, Agnieszka Chalaszczyk ${ }^{1}$, Ernesto Di Cesare ${ }^{1}$, Vincenzo Tombolini ${ }^{4}$, Carlo Masciocchi ${ }^{1}$ and Giovanni Luca Gravina ${ }^{1}$

${ }^{1}$ Division of Radiotherapy, Department of Biotechnological and Applied Clinical Sciences, University of L'Aquila, L'Aquila, Italy;

${ }^{2}$ Division of Medical Physics, San Salvatore

Hospital, L'Aquila, L'Aquila, Italy;

${ }^{3}$ Division of Radiotherapy, San Salvatore Hospital, L'Aquila, L'Aquila, Italy;

${ }^{4}$ Division of Radiotherapy, Department of Radiological, Oncological and Pathological Sciences, Policlinico Umberto I, "Sapienza" University of Rome, Rome, Italy

Background/Aim: Radiation therapy (RT) is a curative treatment modality for localized prostate cancer. Randomized trials suggest that in men with pre-RT genitourinary (GU) and gastrointestinal (GI) morbidity, some hypofractionated regimens giving fewer fractions with higher dose may be associated with increased toxicity. Today, intensity-modulated RT (IMRT) is considered standard, where radiation beams of different shapes and intensities can be delivered from a wide range of angles, thus further decreasing doses to normal organs and likely reducing treatment-related toxicity. At the same time, IMRT with or without image-guided RT (IGRT) seems to decrease post-RT GI and GU toxicity. A limited number of clinical data are available about the incidence of GU and GI toxicity in men undergoing hypofractionated RT (HyRT) and treated by volumetric-modulated arc therapy (VMAT) and IGRT. This study aims to compare the acute GI and GU toxicity of men undergoing HyRT and treated by three-dimensional conformal radiation therapy (3DCRT) or VMAT with daily IGRT. Patients and Methods: A retrospective cohort of 91 men with low/intermediate risk prostate cancer (Pca) was 
Table I. Acute gastrointestinal (GI) toxicity in patients treated with three-dimensional conformal radiation therapy (3DCRT) or volumetric-modulated arc therapy (VMAT).

\begin{tabular}{lcccrc}
\hline & \multicolumn{3}{c}{ RTOG GU acute toxicity } & \\
\cline { 2 - 4 } & \multicolumn{2}{c}{3 DCRT } & \multicolumn{2}{c}{ VMAT } & \\
\cline { 2 - 4 } & No. & $\%$ & No. & $\%$ & \\
\cline { 2 - 4 } & & & & & \\
\hline 1 week post RT & $42 / 70$ & 60 & $19 / 21$ & 90.5 & 0.0087 \\
Grade $\leq 1$ & $28 / 70$ & 40 & $2 / 21$ & 9.5 & \\
Grade $\geq 2$ & & & & & \\
4 weeks post RT & $56 / 70$ & 80 & $21 / 21$ & 100 & 0.034 \\
Grade $\leq 1$ & $14 / 70$ & 20 & $0 / 21$ & 0 & \\
Grade $\geq 2$ & & & & & \\
12 weeks post RT & $65 / 70$ & 92.8 & $21 / 21$ & 100 & 0.586 \\
Grade $\leq 1$ & $5 / 70$ & 7.2 & $0 / 21$ & 0 & \\
Grade $\geq 2$ & & & & & \\
\hline
\end{tabular}

RTOG: Radiation Therapy Oncology Group; GI: gastrointestinal; 3DCRT: three-dimensional conformal radiation therapy; VMAT: volumetric-modulated arc therapy; RT: radiation therapy.

Table II. Acute genitourinary $(G U)$ toxicity in patients treated with three-dimensional conformal radiation therapy (3DCRT) or volumetric-modulated arc therapy (VMAT).

\begin{tabular}{|c|c|c|c|c|c|}
\hline & \multicolumn{4}{|c|}{ RTOG GI acute toxicity } & \multirow{3}{*}{$p$-Value } \\
\hline & \multicolumn{2}{|c|}{ 3DCRT } & \multicolumn{2}{|c|}{ VMAT } & \\
\hline & $\mathrm{n}$ & $\%$ & $\mathrm{n}$ & $\%$ & \\
\hline \multicolumn{6}{|c|}{1 week post RT } \\
\hline Grade $\leq 1$ & $41 / 70$ & 58.6 & $20 / 21$ & 95.3 & 0.0012 \\
\hline Grade $\geq 2$ & $29 / 70$ & 41.4 & $1 / 21$ & 4.7 & \\
\hline \multicolumn{6}{|c|}{4 weeks post RT } \\
\hline Grade $\leq 1$ & $51 / 70$ & 72.8 & $21 / 21$ & 100 & 0.005 \\
\hline Grade $\geq 2$ & $19 / 70$ & 27.2 & $0 / 21$ & 0 & \\
\hline \multicolumn{6}{|c|}{12 weeks post RT } \\
\hline Grade $\leq 1$ & $67 / 70$ & 95.7 & $21 / 21$ & 100 & 1.0 \\
\hline Grade $\geq 2$ & $3 / 70$ & 4.3 & $0 / 21$ & 0 & \\
\hline
\end{tabular}

RTOG: Radiation Therapy Oncology Group; GU: genitourinary; 3DCRT: three-dimensional conformal radiation therapy; VMAT: volumetric-modulated arc therapy; RT: radiation therapy.

selected according to the NCCN criteria between July 2015 and July 2018. Patients underwent a moderate HyRT with a cumulative dose of $60 \mathrm{~Gy}$ delivered in 20 daily fractions. An IGRT strategy based on daily cone-beam CT was adopted in all treated patients according to the latest clinical indications (NCCN guidelines). A weekly clinical assessment during radiation treatment was arranged for the detection of any early toxicity. Men were treated with VMAT $(n=21)$ or with 3DCRT $(n=70)$. The post treatment follow-up visits were performed at 1, 4 and 12 weeks after the end of radiotherapy. The acute GI and GU toxicities were rated according to the Radiation Therapy Oncology Group (RTOG) scale for early side effects (1). Differences in the incidence of toxicities between the two groups were evaluated by the Fisher's exact test. A $p$-value lower than 0.05 was considered statistically significant. Results: One week after RT, a decrease in the rate of Grade 2 or greater acute toxicity was recorded in the group of men treated by VMAT. Interestingly, Grade 2 or more GI and GU toxicity were observed in $41.4 \% v s .4 .7 \%(p=0.0012)$ and in $40 \%$ vs. $9.5 \%(p=0.0087)$ in men treated by 3DCRT and VMAT, respectively. At four weeks after RT, the reduced toxicity in the study group treated with VMAT was also confirmed. The incidence of Grade 2 or greater GI and GU toxicity was $27.2 \%$ and $20 \%$ after 3DCRT and $0 \%$ after VMAT. The decreased Grade 2 or more GI and GU toxicity observed between the two treatments achieved the statistical significance (GI toxicity VMAT vs. 3DCRT: $p=0.005$; GU toxicity VMAT vs. 3DCRT: $p=0.034$ ) (Tables I and II). Conclusion: Men undergoing HyRT with image-guided VMAT experienced a significantly lower GI toxicity 1 and 4 weeks after RT with respect to men treated by 3DCRT. An identical trend was found for GU toxicity one week and 4 weeks after RT. This advantage was not maintained 12 weeks after RT. The early results are encouraging and guarantee the continuation of the study. Advanced radiotherapy techniques like VMAT with daily IGRT should therefore be considered to reduce acute toxicity profile in treatment of low or intermediate risk prostate cancer $(2,3)$. Our results must be considered preliminary and more powered studies will be necessary to investigate this understudied topic.

1 Cox JD, Stetz J and Pajak TF: Toxicity criteria of the Radiation Therapy Oncology Group (RTOG) and the European Organization for Research and Treatment of Cancer (EORTC). Int J Radiat Oncol Biol Phys 31: 13411346, 1995. PMID: 7713792. DOI: 10.1016/03603016(95)00060-C

2 Valeriani M, Bonfili P, Reverberi C, Marinelli L, Ferella L, Minniti G, De Sanctis V, Osti MF, Bonome P, Tronnolone L, Varrassi E, Gravina GL, Franzese P, Tombolini V and Di Staso M: Moderate hypofractionation in patients with low-risk prostate cancer: long-term outcomes. Anticancer Res 38(3): 1671-1676, 2018. PMID: 29491101. DOI: 10.21873 /anticanres. 12400

3 Krupa P, Ticha H, Kazda T, Dymackova R, Zitterbartova J, Odlozilikova A, Kominek L, Bobek L, Kudlacek A and Slampa P: Early toxicity of hypofractionated radiotherapy for prostate cancer. Biomed Pap Med Fac Univ Palacky Olomouc Czech Repub 160(3): 435-441, 2016. PMID: 26948031. DOI: $10.5507 / \mathrm{bp} .2016 .008$ 
107

\section{PERCUTANEOUS IMAGE-GUIDED RADIOFREQUENCY ABLATION FOR CT1A-B RENAL MASSES: A COMPARISON BETWEEN PATIENTS YOUNGER VS. OLDER THAN 65 YEARS}

Francesco Alessandro Mistretta ${ }^{1}$, Giovanni Mauri ${ }^{2}$, Nicolò Piacentini ${ }^{1}$, Gianluca Varano ${ }^{3}$, Andrea Conti ${ }^{1}$, Guido Bonomo ${ }^{3}$, Stefano Luzzago ${ }^{1}$, Paolo Della Vigna ${ }^{3}$, Michele Catellani ${ }^{1}$, Ettore Di Trapani ${ }^{1}$, Matteo Ferro ${ }^{1}$, Gennaro Musi ${ }^{1}$, Giuseppe Renne ${ }^{3}$, Franco Orsi ${ }^{3}$ and Ottavio De Cobelli ${ }^{1}$

${ }^{1}$ Division of Urology, European

Institute of Oncology, Milan, Italy;

${ }^{2}$ Department of Interventional Radiology,

European Institute of Oncology, Milan, Italy;

${ }^{3}$ Department of Pathology, European

Institute of Oncology, Milan, Italy

Aim: To compare the safety and efficacy of percutaneous image-guided radiofrequency ablation (RFA) for cT1a-b renal masses between patients younger $v s$. older than 65 years. Patients and Methods: From January 2008 to June 2015 a total of 152 consecutive patients underwent an image-guided percutaneous RFA. Primary outcomes investigated were technical success, complications, retreatment rate, cancer specific mortality (CSM) and other cause mortality (OCM). Kaplan-Meier plots graphically depicted the recurrence-free survival (RFS) rates. Univariable (ULRM) and multivariable (MLRM) logistic regression models were used to identify predictors of persistency/recurrence of the disease. Results: Of all 152 patients, $66(43 \%)$ and $86(57 \%)$ were younger and older than 65 years, respectively. The overall median followup was 40 months (IQR=28-49). Median tumour diameter was higher in older patients (27 vs. $22 \mathrm{~mm} ; p=0.01)$, compared to the younger ones. No significant differences were identified in median number of masses treated per procedure, T-stage, gender, side of lesion, endophytic i exophytic nature and Padua score, between the tow age groups. Of all 152 patients, $63(41.4 \%)$ younger vs. 74 (48.7\%) older than 65 years $(p=0.06)$ were disease-free after RFA. In $1(0.7 \%)$ younger vs. $10(6.7 \%)$ older than 65 years patients a persistence of disease was identified $(p=0.06)$. All patients received secondary RFAs. Two (1.3\%) younger $v s .2$ $(1.3 \%)$ older than 65 years patients underwent multiple RFAs due to multiple synchronous renal masses. No persistence was described at last control in all of these 15 retreated patients. Eight $(5.3 \%)$ younger vs. $9(5.9 \%)$ older than 65 years patients experienced a recurrence $(p=0.9)$. In KaplanMeier analyses, the 3-year RFS rate was $90.9 \%$ in younger $v s .94 .2 \%$ in older than 65 years group, but failed to reach statistical significance (log rank $=0.7)$. Of these 17 patients, 4 (23.5\%) experienced a distant (adrenal glands or contralateral kidney) recurrence $[1(25 \%)$ patient younger vs. $3(75 \%)$ older than 65 years]. In all these patients, the oncologic control was achieved after a second RFA. No progression or cancer-related deaths were identified. One $(0.7 \%)$ younger $v s$. $3(2.1 \%)$ older than 65 years patients died due to other causes $(p=0.8)$. Of all 152 patients, according to the Society of Interventional Radiology complication scale, 7 (4.6\%) experienced a grade A, $6(3.9 \%)$ a grade C and $2(1.3 \%)$ a grade $\mathrm{D}$ complication. No statistical difference was identified between the two age groups $(p=0.9)$. In MLRM, tumour size $>2.5 \mathrm{~cm}$ resulted the only predictor of persistence/recurrence $(\mathrm{OR}=1.05 ; 95 \% \mathrm{CI}=1.008-1.099 ; p=0.02)$, while age younger $v s$. older than 65 years failed to predict this outcome. Conclusion: Image-guided percutaneous RFA resulted oncologically safe, with a low complication rate, in both patients younger or older than 65 years old. Tumour size $>2.5$ $\mathrm{cm}$ resulted the only predictor of persistency/recurrence of disease, while age younger $v s$. older than 65 years failed to predict this outcome.

\section{8 \\ A COMPREHENSIVE EVALUATION OF SEXUAL DISORDERS FOLLOWING ROBOT- ASSISTED RETROPERITONEAL LYMPH NODE DISSECTION FOR NON-SEMINOMATOUS GERM CELL TUMOUR}

Francesco Alessandro Mistretta, Gennaro Musi, Andrea Conti, Nicolò Piacentini, Stefano Luzzago, Gabriele Cozzi, Roberto Bianchi, Giovanni Cordima, Antonio Brescia, Matteo Ferro and Ottavio De Cobelli

Division of Urology, European Institute of Oncology, Milan, Italy

Aim: Sexual disorders following retroperitoneal pelvic lymph node dissection (RPLND) can affect the quality of life of patients with non-seminomatous germ cell tumour (NSGCT) of testis. The aim of the current study was to investigate several of the possible andrological aspects, which may be influenced by robot-assisted (RA) RPLND. Materials and Methods: Between January 2012 and April 2018, 27 patients underwent RA-RPLND for stage I NSGCT or postchemotherapy (PC) residual mass respectively. In all patients, modified unilateral RPLND template was used. A nervesparing procedure was always attempted. Major variable of interest was the occurrence of erectile dysfunction (ED), premature ejaculation (PE), dry ejaculation (DE), or orgasm alteration. Preoperatively, body mass index (BMI), scholar status, presence and type of preoperative sexual dysfunction (ED, PE, dimorphism, orgasmic alteration), and risk factors for sexual disorder (smoking status, alcohol intake, diabetes/metabolic syndrome, neuroleptic/antiepileptic drugs) 
were investigated. The abridged 5-item version of the International Index of Erectile Function (IIEF-5) was administered pre- and post-operatively. The pre-orchiectomy and post-RA-RPLND sperm count analyses, if performed, were retrieved. All patients were asked to evaluate whether their post-operative experiences in sexual desire, orgasmic functioning and sexual satisfaction were 'better, same, or worse' respect to before RA-RPLND. Lastly, research and eventual obtainment of children was investigated, as well as the fecundation process [sexual intercourse or medically assisted procreation (MAP)]. Results: Of all 27 patients, 9 $(33.3 \%)$ presented an andrological disorder of any type after RA-RPLND. The median number of LN removed was similar for both patient groups who experienced post-operative sexual dysfunctions and who did not (17 vs. 16.5). Low semen volume was present in $4(14.8 \%)$ patients, DE (IIEF$5<25)$ in $2(7.4 \%)$ and ED in $3(11.1 \%)$. No PE or orgasmic alterations were described. Similar median age at surgery (29 vs. 31 years) and BMI (23.8vs. 24.6) were reported for both subgroups. Of all 9 patients presenting post-operative andrological disorders, no one presented any pre-operative risk factor for sexual dysfunction or altered pre-operative spermiogram. Of all these 9 patients, $77.8 \%$ was treated at the beginning of our robotic experience (2012-2015). Of all 27 patients, $5(18.5 \%)$ attempted to have a child after RARPLND. All of these 5 patients were successfully fathered children. Of all 5 patients, $2(40 \%)$ required a MAP. Conclusion: A not negligible number of andrological complications occurred after RA-RPLND, mainly represented by ejaculation disorders, but ED occurrence and overall sexual satisfaction deficit should be definitely considered. No negative impact on fertility was described after RA-RPLND in patients with NSGCT.

\section{0}

\section{A COMPARISON BETWEEN TRANS-RECTAL ULTRASOUND-GUIDED MRI-FUSION VERSUS MRI-COGNITIVE VERSUS STANDARD PROSTATE BIOPSIES PERFORMED AT THE SAME TIME, IN THE SAME PATIENT: CONCORDANCE RATE AND CLINICALLY SIGNIFICANT PROSTATE CANCER DETECTION}

Francesco Alessandro Mistretta ${ }^{1}$, Andrea Conti $^{1}$, Giovanni Cordima $^{1}$, Antonio Brescia ${ }^{1}$, Sarah Alessi ${ }^{2}$, Beatrice Costa ${ }^{1}$, Nicolò Piacentini ${ }^{1}$, Michele Catellani ${ }^{1}$, Paola Pricolo ${ }^{2}$, Stefano Luzzago ${ }^{1}$, Gabriele Cozzi ${ }^{1}$, Roberto Bianchi ${ }^{1}$, Giuseppe Petralia ${ }^{2}$ and Ottavio De Cobelli ${ }^{1}$

${ }^{1}$ Division of Urology, European Institute of Oncology, Milan, Italy;

${ }^{2}$ Division of Radiology, European Institute of Oncology, Milan, Italy
Aim: To test the efficacy of three different prostate biopsy strategies, namely magnetic resonance imaging (MRI)-fusion $v s$. MRI-cognitive $v s$. standard, contextually performed in the same patient. Patients and Methods: Between December 2017 and September 2018, 153 patients underwent a combined trans-rectal ultrasound-guided prostate biopsy composed of 35 MRI-fusion core samples, 3-5 MRI-cognitive core samples, and 12 standard core samples. The number of MRI-targeted (fusion or cognitive) core samples varied according to tumour volume. Primary outcomes investigated were: concordance rate of prostate cancer $(\mathrm{PCa})$ detection between the three strategies and rate of diagnosis of clinically significant $\mathrm{PCa}$, defined as Gleason Score $(\mathrm{GS}) \geq 7$. Complete concordance between the three strategies was defined as: $\mathrm{PCa}$ simultaneously diagnosed with all the three-biopsy strategies or PCa not detected in any strategy. Multivariable logistic regression models (MLRMs) were used to identify preoperative predictors of complete concordance. In MLRMs, covariates consisted of: age, prostate-specific antigen (PSA), PI-RADS score (version 2), prostate volume, surgeon expertise. Results: Of all 153 patients, PCa was diagnosed in 87 (56.9\%) MRI-fusion, 77 (50.3\%) MRI-cognitive, and 74 $(48.3 \%)$ standard biopsies. In 104 (68\%) patients, PCa was diagnosed in at least one biopsy strategy (MRI-fusion and/or MRI-cognitive and/or standard). In 28 (18.3\%) patients, PCa was simultaneously diagnosed in two different biopsy strategies. Complete concordance between the three-biopsy strategies was achieve in $102(66.7 \%)$ patients, $53(34.6 \%)$ simultaneously diagnosed for $\mathrm{PCa}$ in all the three strategies, $49(32.0 \%)$ not diagnosed for PCa in any strategy. No predictors of complete concordance were identified in MLRMs. When only MRI-fusion and standard biopsy strategies were compared, a complete concordance was identified in $116(75.8 \%)$ patients. In MLRMs predicting complete concordance between MRI-fusion and standard biopsy, PI-RADS 4 was an independent predictor of lower rate of complete concordance $(\mathrm{OR}=0.3 ; 95 \% \mathrm{CI}=0.1-0.9 ; p=0.04)$. Of all $104 \mathrm{PCa}$-diagnosed patients, clinically significant $\mathrm{PCa}$ was detected in $61 \mathrm{MRI}$-fusion (58.7\%; 71\% out of $87 \mathrm{PCa}$ positive MRI-fusion biopsies), 55 MRI-cognitive (52.9\%; 78\% out of $77 \mathrm{PCa}$ positive MRI-cognitive biopsies) and 52 standard biopsies $(50.0 \% ; 70 \%$ out of $74 \mathrm{PCa}$ positive standard biopsies). Of all 153 patients, 15 (11\%) were treated with radical prostatectomy (RP). In all of them, clinically significant PCa was diagnosed at final pathology. Among these 15 patients, MRI-fusion failed to detect PCa in $1(6.7 \%)$, MRI-cognitive in $4(26.7 \%)$, and standard biopsy in $3(20 \%)$. Conclusion: Low rate of complete concordance $(66.7 \%)$ was described between the three-biopsy strategies. Higher rates of $\mathrm{PCa}$ detection and clinically significant $\mathrm{PCa}$ were recorded in MRI-fusion patients. In patients with clinically significant PCa at final pathology after RP, MRI-fusion biopsy had higher rate of PCa detection. 
111

\section{DO NUMBER OF BIOPSIES AND PSA DOUBLING TIME ASSOCIATE WITH UPGRADING RECLASSIFICATION IN ACTIVE SURVEILLANCE?}

Fabio Badenchini ${ }^{1}$, Cristina Marenghi ${ }^{1}$, Nicol Nicolai ${ }^{2}$, Barbara Avuzzi ${ }^{3}$, Lara Bellardita ${ }^{1}$, Alessandra Casale ${ }^{4}$, Mario Catanzaro ${ }^{2}$, Maurizio Colecchia ${ }^{5}$, Letizia De Luca ${ }^{1}$, Teresa Di Florio ${ }^{1}$, Simona Donegani ${ }^{1}$, Paola Dordoni ${ }^{1}$, Alberto Macchi ${ }^{2}$, Tiziana Magnani ${ }^{1}$, Simona Massa ${ }^{5}$, Antonella Messina ${ }^{4}$, Sara Morlino ${ }^{3}$, Barbara Noris Chiorda ${ }^{3}$, Tiziana Rancati ${ }^{1}$, Silvia Stagni ${ }^{2}$, Antonio Tesone ${ }^{2}$, Tullio Torelli ${ }^{2}$, Sergio Villa ${ }^{3}$,

Fabiana Zollo ${ }^{1}$, Roberto Salvioni ${ }^{2}$ and Riccardo Valdagni ${ }^{6}$

${ }^{1}$ Prostate Program, Fondazione IRCCS Istituto

Nazionale dei Tumori, Milan, Italy;

${ }^{2}$ Division of Urology, Fondazione IRCCS Istituto

Nazionale dei Tumori, Milan, Italy;

${ }^{3}$ Division of Radiation Oncology 1, Fondazione IRCCS

Istituto Nazionale dei Tumori, Milan, Italy;

${ }^{4}$ Division of Radiology, Fondazione IRCCS Istituto

Nazionale dei Tumori, Milan, Italy;

${ }^{5}$ Division of Pathology, Fondazione IRCCS

Istituto Nazionale dei Tumori, Milan, Italy;

${ }^{6}$ Department of Oncology And Hemato-oncology,

Fondazione IRCCS Istituto Nazionale dei

Tumori, Milan, Italy

Background/Aim: Active Surveillance (AS) is one of the possible choices for patients with very low or low risk prostate cancer. During AS repeated biopsies are performed to promptly detect possible tumor reclassifications and switch to radical treatment within the curability window. We evaluated whether prostate-specific antigen doubling time (PSA-DT) and number of biopsies carried out per patient impact on the chance of upgrading reclassification in twotime windows of observation ( 3 and 5 years) among patients in AS. Patients and Methods: Since 2005, 1,036 patients were enrolled in 2 different protocols of AS (SAINT and PRIAS). In both protocols, eligibility criteria included clinical stage $\leq \mathrm{T} 2 \mathrm{a}$, initial PSA (iPSA) $\leq 10 \mathrm{ng} / \mathrm{ml}$ and Gleason Pattern Score (GPS) $\leq 3+3$; number of admitted positive cores was $\leq 25 \%$ of total cores and a maximum core length containing cancer $\leq 50 \%$ in SAINT protocol, $\leq 2$ positive cores and PSA density $<0.2 \mathrm{ng} / \mathrm{ml} / \mathrm{cm}^{3}$ in PRIAS protocol. Starting from 2016, multiparametric MRI (mpMRI) allowed the inclusion of patients with GPS $3+3$ in PRIAS, regardless of the number of positive cores if target biopsies excluded Gleason $>3$ in mpMRI PIRADS $>2$ lesions. Four PSA determinations and 2 visits were provided per year. Repeat biopsies were scheduled at year 1, 4, 7, and every 5 years in PRIAS protocol and at year 1,2 then every 2 years up to the 8th year and then every 5 years in SAINT protocol.
Extra-biopsies might be ruled by PSA-DT, absolute PSA value, clinical re-staging. Switching to active treatment was advised in case of grade re-classification to GPS $3+4$ or greater, in case of number of positive cores containing GPS $3+3$ exceeding those included in entry criteria (upsizing) and, in the early years of PRIAS study, also advised in case of very unfavorable PSA-DT ( $<3$ years). Since 2016, only patients harboring GPS $3+4$ or greater at repeat biopsy were advised to undergo active treatment. For this analysis twotime windows at the $3 \mathrm{rd}$ and 5 th year ( \pm 6 months) were considered to evaluate the proportion of biopsies with and without upgrading according to PSA-DT and number of performed biopsies. PSA-DT was categorized as very unfavorable ( $<3$ years, VU-PSADT), unfavorable ( $3-10$ years U-PSADT), favorable ( $>10$ years or negative, F-PSADT). Association with upgrading was evaluated with chi-square test. Results: In the 3rd year, 113 patients have been identified undergoing biopsy: 43 (38.05\%) having F-PSADT, $64(56.64 \%)$ having U-PSADT and $6(5.31 \%)$ having VUPSADT; 5 (4.42\%) underwent one biopsy, 79 (69.92\%) underwent 2 biopsies, 28 (24.78\%) underwent 3 biopsies and $1(0.88 \%)$ underwent 4 biopsies. According to PSA-DT, upgrading was detected in 6/43 (13.95\%) with F-PSADT, in $12 / 64(18.75 \%)$ with U-PSADT and in 3/6 (50\%) with VUPSADT. According to number of biopsies, upgrading was detected in 0/5 undergoing one biopsy, 17/79 (21.52\%) 2 biopsies, $4 / 28$ (14.29\%) 3 biopsies and 0/1 4 biopsies. In a 5-year window, 43 patients have been identified undergoing biopsy: $22(51.16 \%)$ having F-PSADT and $21(48.84 \%)$ having U-PSADT while none had VU-PSADT; 1 (2.33\%) underwent one biopsy, 5 (11.62\%) underwent 2 biopsies, 23 (53.49\%) underwent 3 biopsies, 13 (30.23\%) underwent 4 biopsies and $1(2.33 \%) 5$ biopsies. According to PSA-DT, upgrading was detected in $1 / 22(4.55 \%)$ with F-PSADT, and in $6 / 21(28.57 \%)$ with U-PSADT. According to number of biopsies, upgrading was detected in $0 / 1$ undergoing 1 biopsy, 1/5 (20\%) 2 biopsies, 6/23 (26.0\%) 3 biopsies, 0/13 4 biopsies and 0/1 5 biopsies. Neither PSA-DT [chi-squared value 4.52 with 2 degrees of freedom (DF), $p=0.10]$ nor number of biopsies (chi-squared value 2.16 with $3 \mathrm{DF}$, $p=0.54$ ) were significantly associated with upgrading during 3rd year. During 5th year, PSADT showed a slight association with upgrading (chi-squared value 4.45 with 1 DF, $p=0.035$ ), while number of biopsies did not (chi-squared value 4.59 with $4 \mathrm{DF}, p=0.3319)$. Conclusion: Proportion of upgrading did not change between 3rd (18\%) and 5th (16\%) year. During the 5th year, VU-PSA-DT did not occur, but UPSA-DT showed an association with upgrading occurrence. Number of biopsies did not show any association with upgrading in both windows. PSA-DT did not associate with up-grading during 3rd year. Possibly, PSA-DT may be useful in the middle term (5 years) period of observation to suggest extra-biopsy among patients in AS. 
112

\section{LONG-TERM RECLASSIFICATION \\ DURING ACTIVE SURVEILLANCE \\ FOR LOW RISK PROSTATE CANCER: NUMBER OF REPEATED BIOPSIES EFFECT}

Nicola Nicolai ${ }^{1}$, Cristina Marenghi ${ }^{2}$, Fabio Badenchini ${ }^{2}$, Tiziana Magnani ${ }^{2}$, Sergio Villa ${ }^{3}$, Mario Achille Catanzaro ${ }^{1}$, Barbara Avuzzi ${ }^{3}$, Alberto Macchi ${ }^{1}$, Sara Morlino ${ }^{3}$, Silvia Stagni ${ }^{1}$, Barbara Noris Chiorda ${ }^{3}$, Antonio Tesone ${ }^{1}$, Tullio Torelli ${ }^{1}$, Maurizio Colecchia ${ }^{4}$, Alessandra Casale ${ }^{5}$, Antonella Messina ${ }^{5}$, Lara Bellardita ${ }^{2}$, Fabiana Zollo ${ }^{2}$, Letizia De Luca ${ }^{2}$, Paola Dordoni ${ }^{2}$,

Roberto Salvioni ${ }^{6}$ and Riccardo Valdagni ${ }^{3}$

${ }^{1}$ Division of Urology, Fondazione IRCCS

Istituto Nazionale dei Tumori, Milan, Italy;

${ }^{2}$ Prostate Program, Fondazione IRCCS Istituto

Nazionale dei Tumori, Milan, Italy;

${ }^{3}$ Radiation oncology 1, Fondazione IRCCS

Istituto Nazionale dei Tumori, Milan, Italy;

${ }^{4}$ Division of Pathology, Fondazione IRCCS

Istituto Nazionale dei Tumori, Milan, Italy;

${ }^{5}$ Division of Radiology, Fondazione IRCCS

Istituto Nazionale dei Tumori, Milan, Italy;

${ }^{6}$ Division of Urology, Fondazione IRCCS Istituto

Nazionale dei Tumori, Milan, Italy

Background/Aim: Active Surveillance (AS) is a standard option for patients with very low and low risk prostate cancer. During AS repeated biopsies are performed at planned check-point to detect possible tumor reclassification and switch to radical treatment (prostatectomy or radiation). Extra-biopsies could be required if progression is suspected, on the basis of prostate-specific antigen doubling-time (PSA-DT) or digital rectal examination (DRE). We evaluated proportion of reclassification during time, in order to investigate the impact of extra-biopsies (not regularly scheduled by protocol) in detecting upgrading within the window between 7 th and 8 th years. Patients and Methods: Since 2005, 1,036 patients were enrolled in 2 different protocols of AS (SAINT and PRIAS). Eligibility criteria included clinical stage $\leq \mathrm{T} 2 \mathrm{a}$, initial PSA (iPSA) $\leq 10 \mathrm{ng} / \mathrm{ml}$ and Gleason pattern score (GPS) $\leq 3+3$ (both protocols); less than $25 \%$ positive cores with a maximum core length containing cancer $\leq 50 \%$ in SAINT, $\leq 2$ positive cores and PSA density $<0.2 \mathrm{ng} / \mathrm{ml} / \mathrm{cm}^{3}$ in PRIAS. Since 2016, multiparametric magnetic resonance imaging (mpMRI) permitted to have no limits in the number of positive cores if it was negative or target biopsies excluded Gleason 4 whitin PIRADS >2 lesions. Four PSA determinations and 2 visits were provided per year. Scheduled biopsies were repeated at year $1,4,7$, and every 5 years in PRIAS protocol and at year 1,2 then every 2 years up to 8th year

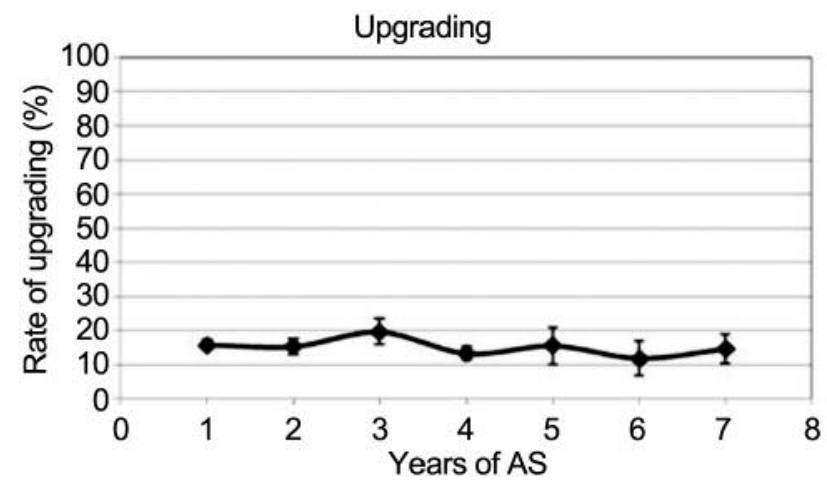

Figure 1. Rate of upgrading during active surveillance (AS).

years and then every 5 years in SAINT protocol. Extrabiopsies might be ruled by PSA-DT $<10$ years, absolute PSA values, clinical re-staging. Switching to active treatment was advised in case of grade re-classification to GPS $3+4$ or greater, in case of number of positive cores containing GPS $3+3$ exceeding those included in entry criteria (upsizing) and, in the early years of PRIAS study, also advised in case of very unfavorable PSA-DT $(<3$ years). Since 2016, when mpMRI was included in AS workup, only patients harbouring GPS $3+4$ or greater at repeat random or targeted biopsies were advised to undergo active treatment. Results: Since 2005, 1,688 biopsies have been performed in 13 years: 258 (15.3\%) underwent reclassification due to upgrading. In the window between 7 th and 8 th year, 80 patients have been identified, and 10 (12\%) of them were upgraded. As many as 140/258 (54.3\%) biopsies recorded upgrading following first year. Nonetheless, proportions of reclassification during the years remained stable, as reported in Figure 1. Notably, no reclassification was recorded beyond 8 years, but 22 patients only reached that follow-up time. Considering the window between 7 th and 8th year, 47/82 (57.3\%) had not extrabiopsies, while $35 / 82(42.7 \%)$ had at least one extra-biopsy. Upgrading occurred in 8/47 (17\%) patients with no extrabiopsies vs. $2 / 35(5.7 \%)$ of those who had an extra-biopsy at least. This difference was not statistically significant at chi-square test [chi-squared value 2.366, degree of freedom (DF) $1, p=0.124]$. Conclusion: Most of the patients $(54.3 \%)$ enrolled in AS had a reclassification due to upgrading just after the first year. Nonetheless, proportion of upgrading reclassifications did not reduce up to the 8th year (ranging between 12 and 16\%). These data need to be confirmed with a greater number of patients on AS with a long-term follow-up. Currently, surveillance pressure is not able to reduce the chance of discovering high-grade disease during observation in this series. The biological and clinical consequences remain uncertain, as no evidence is available 
in illustrating differences between 'early' $v s$. 'late' reclassified diseases. The greater proportion of 'late' reclassification at 7-8 years among patients not undergoing extra-biopsies when compared with those undergoing extrabiopsies was not statistically significant, possibly due to the small number of patients and extra-biopsies. Nonetheless, although the tools in use to suggest extra-biopsy are not reliable, extra-biopsies may anticipate reclassification, select more cases with favourable disease and reduce upgrading in the long term.

\section{3}

\section{SALVAGE RADICAL PROSTATECTOMY FOR RECURRENT PROSTATE CANCER: ONCOLOGICAL OUTCOMES OF A LARGE MULTICENTRE SERIES}

Giancarlo Marra $^{1}$, Paolo Gontero ${ }^{1}$, Paolo Alessio ${ }^{1}$, Giorgio Calleris $^{1}$, Antonino Battaglia ${ }^{1}$, Claudia Filippini ${ }^{2}$, Stefania Munegato ${ }^{1}$, Fernando Munoz ${ }^{3}$, Marco Oderda ${ }^{1}$, Anna Palazzetti ${ }^{1}$, Francesca Pisano ${ }^{1}$, Umberto Ricardi ${ }^{4}$, Estefania Linares ${ }^{5}$, Rafael Sanchez-salas ${ }^{5}$, Ben Challacombe ${ }^{6}$, Paul Cathcart ${ }^{6}$, Prokar Dasgupta ${ }^{6}$, Sanchia Goonewadene ${ }^{6}$, Rick Popert ${ }^{6}$, Declan Cahill ${ }^{7}$, David Gillatt $^{8}$, Raj Persad ${ }^{8}$, Juan Palou ${ }^{9}$, Steven Joniau ${ }^{10}$, Thierry Piechaud ${ }^{11}$, Salvatore Smelzo ${ }^{11}$, Alexandre De La Taille ${ }^{12}$, Morgan Roupret ${ }^{13}$, Simone Albisinni ${ }^{14}$, Roland Van Velthoven ${ }^{14}$, Alessandro Morlacco ${ }^{15}$, Sharma Vidit ${ }^{15}$, Alexander Mottrie ${ }^{16}$, Joseph Smith ${ }^{17}$, Shreyas Joshi ${ }^{17}$, Gabriel Fiscus ${ }^{17}$, Andre Berger18, Monish Aron ${ }^{18}$, Andre Abreu ${ }^{18}$, Inderbir S. Gill ${ }^{18}$, Henk Van Der Poel ${ }^{19}$, Derya Tilki ${ }^{20}$, Nathan Lawrentschuk ${ }^{21}$, Declan G. Murphy ${ }^{21}$, Gordon Leung ${ }^{22}$, John Davis ${ }^{22}$ and Robert Jeffrey Karnes ${ }^{15}$

${ }^{1}$ Department of Surgery, Urology, University of Turin, AOU Città della Salute e della Scienza, Turin, Italy;

${ }^{2}$ Department of Statistics, University of Studies of Turin, Turin, Italy;

${ }^{3}$ Department of Urology, Parini Hospital, Aosta, Italy; ${ }^{4}$ Department of Radiotherapy, University of Turin, AOU Città della Salute e della Scienza, Turin, Italy; ${ }^{5}$ Department of Urology, Institut Mutualiste Montsouris, Paris, France;

${ }^{6}$ Department of Urology, Urology Centre, Guy's Hospital, London, UK;

${ }^{7}$ Department of Urology, Royal Marsden

Hospital, London, UK;

${ }^{8}$ Department of Urology, Bristol NHS

Foundation Trust, Bristol, UK;
${ }^{9}$ Department of Urology, Fundaciò Puigvert, Barcelona, Spain;

${ }^{10}$ Department of Urology, Leuven University Hospitals, Leuven, Belgium;

${ }^{11}$ Department of Urology, Clinique Saint

Augustin, Bordeaux, France;

${ }^{12}$ Department of Urology, CHU Mondor, Créteil, France;

${ }^{13}$ Department of Urology, Sorbonne Université, GRC N ${ }^{\circ}$, ONCOTYPE-URO, AP-HP, Hôpital

Pitié-Salpêtrière, Paris, France;

${ }^{14}$ Department of Urology, Institut Jules Bordet, Université

Libre de Bruxelles, Bruxelles, Belgium;

${ }^{15}$ Department of Urology, Mayo Clinic,

Rochester, MN, U.S.A.;

${ }^{16}$ Department of Urology, OLV Hospital, Aalst, Belgium;

${ }^{17}$ Department of Urology, Vanderbilt University, Medical Center North, Nashville, TN, U.S.A.;

${ }^{18}$ Department of Urology, USC Norris Comprehensive Cancer Center and Hospital, University of Southern California, Los Angeles, CA, U.S.A.;

${ }^{19}$ Department of Urology, Netherlands Cancer Institute, Amsterdam, The Netherlands; ${ }^{20}$ Department of Urology, University Hospital Hamburg-Eppendorf, Hamburg, Germany;

${ }^{21}$ Department of Urology, Peter MacCallum Cancer Centre, Melbourne, Victoria, Australia; ${ }^{22}$ Department of Urology, Division Of Surgery, The University of Texas MD Anderson Cancer Center, Houston, TX, U.S.A.

Background/Aim: Salvage radical prostatectomy (sRP) is a curative option in prostate cancer (PCa) patients with biochemical recurrence (BCR) after primary treatment. High complication rates and poor outcomes were reported by older series. Contemporary oncological outcomes of sRP were investigated in large recent series. Materials and Methods: Six hunded and fifteen patients with BCR underwent sRP at 18 tertiary referral centres, between 2000 and 2016. Pre-, intra-, and post-surgery clinical and histological data were retrospectively registered. We excluded patients with a followup shorter than 6 months or insufficient data. Continuous variables were compared using Wilcoxon-Mann-Whitney test; differences in categorical variables were assessed by Chisquare or Fisher's exact tests. Results: Inclusion criteria were reached by 349 men. At first diagnosis, PCa had been treated by radiotherapy, brachytherapy, cryotherapy, high-intensity focused ultrasound (HIFU) or other primary treatments (in $3.3 \%$ of the cases). Age at sRP was 66.2 years $(\mathrm{IQ}=62.0-70.3)$, whilst PSA before surgery was $4.3 \mathrm{ng} / \mathrm{ml}$ (IQ=2.5-7.3). No patient had radiological evidence of extra-nodal metastases before salvage treatment and $6.87 \%(n=24)$ had castration resistant prostate cancer (CRPC). More than half of the patients $(64 \%)$ had an ASA score $\leq 2$. Extended or super- 
extended lymphadenectomy patterns (the latter including retroperitoneal nodes) were performed in 53 (15.18\%) patients. A nerve-sparing (full or partial) approach was attempted in $27(7.7 \%)$ patients. Median operating time was $187 \mathrm{~min}(\mathrm{IQR}=147-247)$. Median blood loss was $300 \mathrm{ml}$ $(\mathrm{IQR}=150-500)$. At final pathology, Gleason score (GS) $\leq 7$ was present in $56.6 \%$, and organ-confined disease (T stage $=2$ ) was diagnosed in $40.9 \%$. One out of 6 patients had N1 disease ( $15.75 \%$ nodal involvement). Positive surgical margins were observed in $33.8 \%(\mathrm{n}=118)$. After a 3.24-year follow-up $(\mathrm{IQ}=1.88-5.34), 48.3 \%$ of the men were BCR-free, and $14.9 \%$ had CRPC. Five-year BCR-free, cancer-specific and overall survival were $35.0 \%, 94.8 \%$ and $91.7 \%$, respectively. Conclusion: Salvage surgery may entail promising oncological results for recurrent $\mathrm{PCa}$, in the short-to-medium term. However, rates of positive surgical margins and recurrence are considerable. To investigate the benefits of sRP and to improve criteria of patient selection long-term prospective studies are needed.

\section{4 \\ CHOOSING BEST CANDIDATES FOR SALVAGE RADICAL PROSTATECTOMY: EAU GUIDELINES-COMPLIANT VS. NON- COMPLIANT PATIENTS OUTCOMES}

Giancarlo Marra $^{1}$, Paolo Gontero ${ }^{1}$, Paolo Alessio ${ }^{1}$, Giorgio Calleris $^{1}$, Antonino Battaglia ${ }^{1}$, Claudia Filippini ${ }^{2}$, Stefania Munegato ${ }^{1}$, Fernando Munoz $^{3}$, Marco Oderda ${ }^{1}$, Anna Palazzetti ${ }^{1}$, Francesca Pisano ${ }^{1}$, Umberto Ricardi ${ }^{4}$, Estefania Linares ${ }^{5}$, Rafael Sanchez-salas ${ }^{5}$, Ben Challacombe ${ }^{6}$, Paul Cathcart ${ }^{6}$, Prokar Dasgupta ${ }^{6}$, Sanchia Goonewadene ${ }^{6}$, Rick Popert ${ }^{6}$, Declan Cahill ${ }^{7}$, David Gillatt $^{8}$, Raj Persad ${ }^{8}$, Juan Palou ${ }^{9}$, Steven Joniau ${ }^{10}$, Thierry Piechaud ${ }^{11}$, Salvatore Smelzo ${ }^{11}$,

Alexandre De La Taille ${ }^{12}$, Giorgio Gandaglia ${ }^{13}$, Morgan Roupret ${ }^{14}$, Simone Albisinni ${ }^{15}$, Roland Van Velthoven ${ }^{15}$, Alessandro Morlacco ${ }^{16}$, Sharma Vidit ${ }^{16}$, Alexander Mottrie ${ }^{17}$, Joseph Smith ${ }^{18}$, Shreyas Joshi ${ }^{18}$, Gabriel Fiscus ${ }^{18}$, Andre Berger ${ }^{19}$, Monish Aron ${ }^{19}$, Andre Abreu ${ }^{19}$, Inderbir S. Gill ${ }^{19}$, Henk Van Der Poel ${ }^{20}$, Derya Tilki ${ }^{21}$, Nathan Lawrentschuk $^{22}$, Declan G. Murphy ${ }^{22}$, Gordon Leung ${ }^{23}$, John Davis ${ }^{23}$ and Robert Jeffrey Karnes ${ }^{16}$

${ }^{1}$ Department of Surgery, Urology, University of Turin, AOU Città della Salute e della

Scienza, Turin, Italy;

${ }^{2}$ Department of Statistics, University

of Studies of Turin, Turin, Italy;

${ }^{3}$ Department of Urology, Parini Hospital, Aosta, Italy; ${ }^{4}$ Department of Radiotherapy, University of Turin, AOU Città della Salute e della Scienza, Turin, Italy;
${ }^{5}$ Department of Urology, Institut

Mutualiste Montsouris, Paris, France;

${ }^{6}$ Department of Urology, Urology Centre,

Guy's Hospital, London, U.K.;

${ }^{7}$ Department of Urology, Royal Marsden

Hospital, London, U.K.;

${ }^{8}$ Department of Urology, Bristol NHS

Foundation Trust, Bristol, U.K.;

${ }^{9}$ Department of Urology, Fundaciò

Puigvert, Barcelona, Spain;

${ }^{10}$ Department of Urology, Leuven University

Hospitals, Leuven, Belgium;

${ }^{11}$ Department of Urology, Clinique

Saint Augustin, Bordeaux, France;

${ }^{12}$ Department of Urology, CHU Mondor, Créteil, France;

${ }^{13}$ Department of Urology, OLV Hospital, Aalst, Belgium;

${ }^{14}$ Department of Urology, Sorbonne Université, GRC N ${ }^{\circ}$, ONCOTYPE-URO, AP-HP, Hôpital

Pitié-Salpêtrière, Paris, France;

${ }^{15}$ Department of Urology, Institut Jules Bordet,

Université Libre de Bruxelles, Bruxelles, Belgium;

${ }^{16}$ Department of Urology, Mayo Clinic,

Rochester, MN, U.S.A.;

${ }^{17}$ Department of Urology, OLV Hospital, Aalst, Belgium;

${ }^{18}$ Department of Urology, Vanderbilt University, Medical

Center North, Nashville, TN, U.S.A.;

${ }^{19}$ Department of Urology, USC Norris Comprehensive

Cancer Center and Hospital, University of

Southern California, CA, U.S.A.;

${ }^{20}$ Department of Urology, Netherlands

Cancer Institute, Amsterdam, The Netherlands;

${ }^{21}$ Department of Urology, University Hospital

Hamburg-Eppendorf, Hamburg, Germany;

${ }^{22}$ Department of Urology, Peter MacCallum

Cancer Centre, Melbourne, Victoria, Australia;

${ }^{23}$ Department of Urology, Division of Surgery, The

University of Texas MD Anderson Cancer

Center, Houston, TX, U.S.A.

Background/Aim: Salvage radical prostatectomy (sRP) can represent a curative treatment for patients experiencing biochemical recurrence (BCR) after primary therapy. Accurate patient selection is paramount to maximize expected benefits of this procedure. EAU guidelines state that sRP candidates should have low comorbidities, pre-sRP prostate-specific antigen (PSA) $<10 \mathrm{ng} / \mathrm{ml}$, pre-sRP biopsy Gleason score (GS) $\leq 8$, no evidence of lymph-node or extra-nodal metastases and previous organ-confined disease. Histological and oncological results between patients compliant and non-compliant with these requirements were compared in this study. Materials and Methods: We retrospectively selected 73 fully EAU-compliant (lower risk, group A) and 236 non-EAU-compliant patients (higher risk, group B: missing at least one of the above- 
mentioned characteristics) from a dataset of 615 sRP performed between 2000 and 2016 at 18 tertiary referral centres. Clinical and histological data were registered before, during, and after sRP. A follow-up $<6$ months or unavailability of the data were exclusion criteria. Continuous variables were compared using Wilcoxon-Mann-Whitney test; differences in categorical variables were assessed by Chi-square or Fisher's exact tests. Results: The two groups were similar considering median age at sRP (65.57 vs. 66.91 years, $p=0.11)$ and followup duration (3.43vs. 3.12 years, $p=0.16$ ). As expected, presalvage surgery PSA was significantly higher among higher risk patients $[5.0 \mathrm{ng} / \mathrm{ml}(\mathrm{IQR}=2.5-5.4) v s .3 .8 \mathrm{ng} / \mathrm{ml}(\mathrm{IQR}=2.6-$ 9.0) $p=0.01]$, as well as ASA score and GS distribution at confirmatory biopsy. In group A vs. group B, organ-confined disease at sRP (pT2) was encountered in $68.5 \% \mathrm{vs} .35 .9 \%$ $(p<0.01)$, lymph-node metastases in $7.8 \% v s .23 .5 \%(p<0.01)$, and GS $\geq 8$ disease in $8.8 \%$ vs. $56.1 \%(p<0.01)$, respectively. Positive surgical margins were more frequent in higher risk patients $(43 \%$ vs. $27 \%, p=0.02)$. group A showed a nearly doubled BCR-free survival at last follow-up (64.4\% vs. $37.9 \%, p>0.01)$. Besides, no differences in survival were demonstrated; $94.5 \%$ vs. $93.6 \%$ patients alive for group A vs. B, respectively. Conclusion: Three years after sRP, $64.4 \%$ of men fully-compliant to EAU selection criteria (relatively lowrisk disease) are still disease-free. On the grounds of these results, potentially-curative surgical salvage treatment should probably not be precluded upfront for accurately selected patients, in whom the expected oncological benefit should be weighed against non-negligible complication rates and potential functional problems. Large long-term series are needed to confirm sRP benefits and to enhance patient selection.

\section{5 \\ ROLE OF MULTI-PARAMETRIC MRI IN ACTIVE SURVEILLANCE PROTOCOL RECLASSIFICATION RISK}

\author{
Mario Achille Catanzaro ${ }^{1}$, Badenchini Fabio ${ }^{2}$, \\ Cristina Marenghi ${ }^{2}$, Barbara Avuzzi ${ }^{3}$, Lara Bellardita ${ }^{2}$, \\ Alessandra Casale ${ }^{4}$, Maurizio Colecchia ${ }^{5}$, \\ Letizia De Luca ${ }^{2}$, Teresa Di Florio ${ }^{2}$, Simona Donegani ${ }^{2}$, \\ Paola Dordoni ${ }^{2}$, Rodolfo Lanocita ${ }^{4}$, Alberto Macchi ${ }^{1}$, \\ Tiziana Magnani ${ }^{2}$, Simona Massa $^{5}$, Antonella Messina ${ }^{4}$, \\ Sara Morlino $^{3}$, Nicola Nicolai ${ }^{1}$, Barbara Noris Chiorda ${ }^{3}$, \\ Tiziana Rancati ${ }^{2}$, Silvia Stagni ${ }^{1}$, Antonio Tesone ${ }^{1}$, \\ Tullio Torelli ${ }^{1}$, Sergio Villa ${ }^{3}$, Fabiana Zollo ${ }^{2}$, \\ Roberto Salvioni ${ }^{1}$ and Riccardo Valdagni ${ }^{6}$ \\ ${ }^{1}$ Division of Urology, Fondazione IRCCS, Istituto \\ Nazionale dei Tumori, Milan, Italy; \\ ${ }^{2}$ Prostate Program, Fondazione IRCCS, Istituto \\ Nazionale dei Tumori, Milan, Italy;
}

${ }^{3}$ Division of Radiation Oncology 1, Fondazione IRCCS, Istituto Nazionale dei Tumori, Milan, Italy; ${ }^{4}$ Division of Radiology, Fondazione IRCCS, Istituto Nazionale dei Tumori, Milan, Italy; ${ }^{5}$ Division of Pathology, Fondazione IRCCS, Istituto Nazionale dei Tumori, Milan, Italy; ${ }^{6}$ Division of Oncology and Haemato-oncology, Fondazione IRCCS, Istituto Nazionale dei Tumori, Milan, Italy

Background/Aim: Active surveillance (AS) for prostate cancer (PCa) is offered to patients with low risk prostate cancer to avoid or defer radical treatment side effects. Since 2005 our institution followed patients who choose AS and prospetic data were collected. In 2016 prostatic multi parametric magnetic resonance imaging (mpMRI) and fusion biopsies were introduced in AS work-up to identify clinically significant foci at inclusion and during surveillance. In this preliminary work we evaluated the association between upgrading reclassification at repeated biopsies and mpMRI parameters. Materials and Methods: Since 2005 AS was proposed to patients with very low or low risk tumor and patients were enrolled in the most suitable protocol (institutional protocol SAINT or International protocol PRIAS). In 2016, a new protocol was introduced (SPRINT) and $\mathrm{mpMRI}$ were performed to improve inclusion criteria and follow-up monitoring. Eligibility criteria included clinical stage $\leq \mathrm{T} 2 \mathrm{a}$, initial prostate-specific antigen (iPSA) $\leq 10 \mathrm{ng} / \mathrm{ml}$ and Gleason pattern score (GPS) $\leq 3+3, \leq 25 \%$ positive cores with a maximum core length containing cancer $\leq 50 \%$ (SAINT) or $\leq 2$ positive cores and PSA density $<0.2$ $\mathrm{ng} / \mathrm{ml} / \mathrm{cm} 3$ (PRIAS). The introduction of mpMRI and fusion biopsies permits the inclusion of patients with more than 2 positive cores if GPS was $3+3$. AS scheduled PSA every 3 months, digital rectal examination every 6 month, repeat biopsies at year 1, 4, 7, and every 5 years in PRIAS protocol and at year 1, 2 then every 2 years up to 8 th year and then every 5 years in SAINT protocol. Unplanned mpMRI were performed in SAINT and PRIAS protocols if progression was suspected. Differently, SPRINT protocol introduced mpMRI at inclusion (+/-6 months) and during follow-up with a modulated schedule based on the result of the previous examination. mpMRIs were evaluated using PiRads V2 system. Patients who scored a PiRads $\geq 3$ at mpMRI performed a fusion target biopsy on suspected lesions. Standard mapping for biopsies consisted in 2-4 target and 12 random samples. Patients who performed mpMRI and/or fusion biopsy in different institute were also included in the analysis. Associations between upgrading at repeated biopsy and mpMRI parameters were evaluated using Chi-square test. Logistical regression was performed to identify possible predictors of reclassification. Results: A total of 96 cases were considered for the analysis. Median volume at mpMRI was $50 \mathrm{ml}$ (range=17-155), patients had a median number of 
2 lesions (range $=1-4$ ), median higher ADC was 0.67 (range $=0.45-0.9)$, median diameter of lesion was $0.9 \mathrm{~cm}$ (range $=0.5-2.8)$. Seven patients scored a PiRads 5, 40 resulted to have a PiRads 4 and 44 had a Pirads 3. Seventeen patients had at least 1 lesion in transitional zone, 36 in peripheral area and 24 in both areas. Forty-nine patients scored a GPS $3+3$ at biopsy, 12 had GPS $3+4,5$ scored a GPS 4+3, and 6 patients scored a GPS 4+4 (34, 9, 3 and 5 considering only target cores). Association with upgrading was detected considering maximum lesions diameter $(p=0.0228)$ with a significant trend that suggests increased drop out in patients with greater lesions $(p=0.0044)$. Association with PiRads 5 was also significant (PiRads 5 vs. PiRads $<5, p=0.0311$ ). Considering upgrading exclusively in target cores association with the maximum diameter of lesion resulted to be borderline $(p=0.0586)$ with a confirmed trend $(p=0.019)$, while association with PiRads was confirmed $(p=0.0311)$. No other variables resulted to be associated to upgrading at rebiopsy after mpMRI. Univariate logistic regression confirmed PiRads 5 and maximum diameter of the lesion ( $p=0.0455$ and 0.0069 respectively; $p=0.0142$ and $p=0.0093$ if only target cores were considered). Conclusion: This preliminary work suggests fusion PiRads 5 and maximum diameter of MRI lesions as the only two parameters predicting Gleason upgrading to pattern 4 in our AS protocol.

\section{6 \\ A 13 YEAR EXPERIENCE OF PROSTATE HDR BRACHYTHERAPY: ANALYSIS OF OUTCOME AND TOXICITY}

Carlo Pietro Soatti ${ }^{1}$, Durim Delishaj ${ }^{1}$, Cristina Frigerio ${ }^{2}$, Romerai D'amico ${ }^{1}$, Francesco Bonsignore ${ }^{2}$, Ilaria Costanza Fumagalli ${ }^{3}$, Giuseppe De Nobili ${ }^{1}$, Alessandra Cocchi ${ }^{1}$, Alessandra Vola ${ }^{1}$, Giulia Sangalli ${ }^{2}$, Fausto Declich ${ }^{2}$ and Alessandro Colombo ${ }^{1}$

${ }^{1}$ Department of Radiation Oncology, ASST

LECCO, Lecco, Italy;

${ }^{2}$ Medical Physics Unit, ASST LECCO, Lecco, Italy;

${ }^{3}$ Department ofRadiation Oncology, University

of Milano-Bicocca, Milan, Italy

Aim: To evaluate clinical outcomes, toxicity and dosimetric aspects in patients affected by localized prostate cancer treated with 3D conformal high dose rate (HDR) brachytherapy (BRT) as monotherapy (1-3). Materials and Methods: From March 2004 to October 2017, 277 patients with prostate cancer (T1c-T2cN0M0) were treated in our institute using 3D conformal HDR brachytherapy as monotherapy with a temporary implant. The mean age was 67 years with a range of 47-81 years. Of them, 116 patients were low risk, 145 at intermediate risk, and 15 at high risk. Overall, 154 patients received $38 \mathrm{~Gy}$ in 4 fractions (2 fractions/day in 2 days), 36 patients received $27 \mathrm{~Gy}$ in 2 fractions ( 1 fraction/day), and 87 patients received 19 Gy in 1 fraction. The treatment plan was elaborated using CT based software to perform 3D conformal dose planning aided by an inverse planning algorithm using these dosimetric constraints for organ at risk (OAR): dose received by $2 \mathrm{cc}$ of rectum (D2cc) $<75 \%$ of prescription dose (PD); D2cc of bladder $<80 \%$ PD. For the urethra: the dose received by $1 \%$ of volume $(\mathrm{D} 1 \%)<115 \% \mathrm{PD}$ and $\mathrm{D} 10 \%$ $<110 \%$ PD. The prescription for the target was D90\% $>95 \%$ PD. Results: After a median follow-up of 6 years (range=6160 months) overall survival and cancer-specific survival rates were $90 \%$ and $97 \%$ respectively. Biochemical disease-free rate resulted $78 \%$; for low and intermediate risk biochemical free disease rate was $85 \%$, whereas for high risk disease was $62 \%$. Regarding dosimetric aspects, we obtained satisfactory dose distributions in terms of planning target volume (PTV) coverage (D90\% $>100 \%$ PD), with a strict respect of OAR constraints. Genitourinary (GU) and gastrointestinal (GI) acute toxicity $>\mathrm{G} 2$ toxicity appeared in $28 \%$ of patients. Late toxicity $>\mathrm{G} 2$ was very low $(2.2 \%)$, while only 3 patients reported G3 late toxicity $(0.8 \%)$, which consisted in GU toxicity. Conclusions: With a median follow-up of 6 years, HDR BRT was shown as a valid treatment modality for patients with localized prostate cancer in terms of both biochemical and local control, as well as toxicity. Future studies can be addressed to evaluate the quality of life in this subset of patients.

1 Bachand F, Martin AG, Beaulieu L, Harel F and Vigneault E: An eight-year experience of HDR brachytherapy boost for localized prostate cancer: biopsy and PSA outcome. Int J Radiat Oncol Biol Phys 73: 679-684, 2009. PMID: 18963537. DOI: 10.1016/j.ijrobp.2008.05.003

2 Chin J, Rumble RB, Kollmeier M, Heath E, Efstathiou J, Dorff T, Berman B, Feifer A, Jacques A and Loblaw DA: Brachytherapy for patients with prostate cancer: American Society of Clinical Oncology/Cancer Care Ontario Joint Guideline Update. J Clin Oncol 35: 1737-1743, 2017. PMID: 28346805. DOI: 10.1200/JCO.2016.72.0466

3 Yaxley JW, Lah K, Yaxley JP, Gardiner RA, Samaratunga $\mathrm{H}$ and MacKean J: Long-term outcomes of high-dose-rate brachytherapy for intermediate- and high-risk prostate cancer with a median follow-up of 10 years. BJU Int 120 : 56-60, 2017. PMID: 27628127. DOI: 10.1111/bju.13659
117
CLINICAL OUTCOMES AND PROGNOSTIC FACTORS IN PATIENTS AFFECTED BY LOCALIZED PROSTATE CANCER TREATED HDR BRACHYTHERAPY 
Carlo Pietro Soatti ${ }^{1}$, Durim Delishaj ${ }^{1}$, Cristina Frigerio ${ }^{2}$, Romerai D'amico ${ }^{1}$, Francesco Bonsignore ${ }^{2}$, Ilaria Costanza Fumagalli ${ }^{1}$, Giuseppe De Nobili ${ }^{1}$, Alessandra Cocchi ${ }^{1}$, Alessandra Vola ${ }^{1}$, Giulia Sangalli2, Fausto Declich ${ }^{2}$ and Alessandro Colombo ${ }^{1}$

${ }^{1}$ Radiation Oncology Department, ASST LECCO, Lecco, Italy;

${ }^{2}$ Medical Physics Unit, ASST LECCO, Lecco, Italy

Aim: To evaluate clinical outcomes and prognostic factors in patients affected by localized prostate cancer treated with 3D Conformal high dose rate (HDR) brachytherapy (BT) as monotherapy (1-3). Materials and Methods: Between March 2004 and October 2017, 277 patients with localized prostate cancer (T1c-T2cNOM0) were treated in our institute using 3D conformal HDR BT with a temporary implant. The mean age was 67 years (range $=47-81$ ). Of them, 166 patients were low risk, 145 intermediate risk, and 15 high risk. Overall, 154 patients received $38 \mathrm{~Gy}$ in 4 fractions ( 2 fractions/day in 2 days), 36 patients received $27 \mathrm{~Gy}$ in two fractions (1 fraction/day) and 87 patients received $19 \mathrm{~Gy}$ in one fraction. The treatment plan was elaborated using computed tomography (CT) based software to perform 3D conformal dose planning aided by an inverse planning algorithm using these dosimetric constraints for organ at risk (OAR): dose received by $2 \mathrm{cc}$ of rectum (D2cc) $<75 \%$ of prescription dose (PD); D2cc of bladder $<80 \% \mathrm{PD}$. For the urethra: the dose received by $1 \%$ of volume $(\mathrm{D} 1 \%)<115 \% \mathrm{PD}$ and D10\%<110\%PD. The prescription for the target was D $90 \%>95 \%$ PD. Results: Overall survival and cancer specific survival rates were $90 \%$ and $97 \%$ respectively. The median follow-up was 6 years (range=6-160 months) and biochemical-free disease (BFD) rate was $78 \%$. Patients with low and intermediate risk disease had one advantage in terms of BFD compared to patients with high risk disease ( $p=0.04$, $\mathrm{HR}=2.453,95 \% \mathrm{CI}=1.3113-4.543)$. Also, in patients with initial prostate-specific antigen (iPSA) $<9.5 \mathrm{ng} / \mathrm{ml}$ there was one advantage in terms of BFD compared to patients with $\mathrm{iPSA} \geq 9.5 \quad(p=0.022, \quad \mathrm{HR}=2.042, \quad 95 \% \mathrm{CI}=1.123-4.081)$. Moreover, patients who reached a nadir of PSA $<0.2 \mathrm{ng} / \mathrm{ml}$ and had a PSA value $<0.5 \mathrm{ng} / \mathrm{ml} 3$ months after BT treatment had a benefit in terms BFD $(p=0.003$ and $p=0.001$, respectively). In the same way, patients who reached the nadir within 12 months after BT treatment reported a statistically significant advantage in terms of biochemical recurrence $(p=0.01)$. Patients treated with $38 \mathrm{~Gy}$ in 4 fractions or 27 Gy in 2 fractions showed a benefit in terms of BFD compared to patients treated with a total dose of 19 Gy in one fraction $(p=0.0001, \mathrm{HR}=6.813,95 \% \mathrm{CI}=3.833$ 11.981). Finally, patients with low-intermediate risk disease had an advantage in terms of OS compared to patients with high risk $(p=0.034)$. There were not statistically significant differences regarding the analyzed risk factors and overall survival. Conclusion: High risk disease, iPSA $<9.5 \mathrm{ng} / \mathrm{ml}$, nadir of PSA $<0.2 \mathrm{ng} / \mathrm{ml}, \mathrm{PSA}<0.5 \mathrm{ng} / \mathrm{ml}$ three months after BT, NADIR reached within 12 months after BT, and total prescribed doses were prognostic factors regarding bochemical recurrences. High risk disease was the only prognostic factor for overall survival.

1 Bachand F, Martin AG, Beaulieu L, Harel F and Vigneault E: An eight-year experience of HDR brachytherapy boost for localized prostate cancer: biopsy and PSA outcome. Int J Radiat Oncol Biol Phys 73: 679-684, 2009. PMID: 18963537. DOI: 10.1016/j.ijrobp.2008.05.003

2 Chin J, Rumble RB, Kollmeier M, Heath E, Efstathiou J, Dorff T, Berman B, Feifer A, Jacques A and Loblaw DA: Brachytherapy for patients with prostate cancer: American Society of Clinical Oncology/Cancer Care Ontario Joint Guideline Update. J Clin Oncol 35: 1737-1743, 2017. PMID: 28346805. DOI: 10.1200/JCO.2016.72.0466

3 Yaxley JW, Lah K, Yaxley JP, Gardiner RA, Samaratunga $\mathrm{H}$ and MacKean J: Long-term outcomes of high-dose-rate brachytherapy for intermediate- and high-risk prostate cancer with a median follow-up of 10 years. BJU Int 120: 56-60, 2017. PMID: 27628127. DOI: 10.1111/bju.13659

\section{8 \\ IMMUNHISTOCHEMICAL EXPRESSION OF HER-2 IN BLADDER CANCER DOES NOT ALWAYS MATCH WITH HER2 GENE AMPLIFICATION BY IN SITU HYBRIDIZATION}

Tania Franceschini, Francesca Giunchi, Elisa Capizzi, Stefano Zagnoni and Michelangelo Fiorentino

Laboratory of Oncologic Molecular Pathology, Department of Pathology, St. Orsola Teaching Hospital, University of Bologna, Bologna, Italy

Background/Aim: Overexpression of the HER-2 oncogene has been described in invasive bladder cancer and represents a potential therapeutic target for patients with advanced disease. Overexpression, amplification and mutations of HER2 protein have been assessed so far by immunohistochemistry (IHC), chromogenic in situ hybridization (ISH) and sequencing, but only in bladder cancer with micro-papillary histology. Here we evaluated the IHC overexpression together with the amplification of HER2 in a cohort of patients covering the whole spectrum of bladder cancer histotypes, using a tissue microarray (TMA) strategy. Materials and Methods: We selected 66 patients who underwent to radical cystectomy for muscle-invasive bladder cancer with usual and special types urothelial carcinoma. A TMA was built containing 3 tumor cores for 
each case. IHC was performed on TMA slides using the 4B5 anti-HER-2 antibody and ISH was performed using the INFORM HER2 Dual ISH DNA probe cocktail assay both on a Ventana Bench Mark Ultra automatic instrument. Scoring was performed according to the revised ASCO/CAP guidelines for HER2 amplification in breast cancer. Results: The histological review incorporated the whole spectrum of histological bladder cancer histotypes including 9 cases with micropapillary histology. IHC for HER2 was scored as 3+ in $3 / 66$ cases $(5 \%), 2+$ in 1 case $(2 \%)$ and $1+$ in 3 cases $(5 \%)$. The ISH analysis revealed an amplification (HER2/CEP17 ratio $\geq 2$ ) in $8 / 66$ cases $(12 \%)$. In particular the 3 cases with $3+$ IHC and in the 3 cases with $1+$ IHC also showed amplification of HER2. In addition, we found the amplification of HER2 in 2 cases with IHC score 0 . Conclusion: HER2 was overexpressed in at least $12 \%$ of invasive bladder cancer with various histotypes. IHC and ISH analyses were discrepant with 5 cases with clear HER2 amplification and weak or absent IHC immunostaining. Hence, HER2 is a potential target of therapy in advanced bladder cancer of all histotypes and ISH should be performed besides IHC to assess gene amplification.

\section{9 \\ RADIATION TREATMENT IN PROSTATE CANCER: COMPARISON BETWEEN DIFFERENT DELIVERY TECHNIQUES AND FRACTIONATION SCHEDULES}

Jacopo De Robbio, Mara Caroprese, Esmeralda Scipilliti, Ida Rosalia Scognamiglio, Caterina Oliviero, Stefania Clemente, Antonio Farella, Immacolata Lanzini, Manuel Conson and Roberto Pacelli

Department of Advanced Biomedical Sciences, University "Federico II" School of Medicine, Naples, Italy

Background/Aim: Prostate cancer (PC) is one of the most commonly diagnosed malignancies in men worldwide. Due to wider prostate specific antigen (PSA) use there is an increase of patients diagnosed at an early stage of disease. Currently, radical radiation therapy (RT), radical RT coupled to androgen deprivation therapy (ADT), and post-radical prostatectomy adjuvant RT are the main treatment modalities for most patients with localized or locally advanced PC.

To decrease the risk of toxicity to healthy tissues, several distinct techniques, such as 3D-conformal radiation therapy (3D-CRT), intensity modulated radiation therapy (IMRT) or volumetric modulated arc therapy (VMAT), have been developed, that increase the possibility of conformity of the radiation beam and reduce the margins of planning target volume, ameliorate the therapeutic index. The objective of this study was to compare the toxicity profile of different RT technique and fractionation in patients treated for prostate cancer. Acute gastrointestinal (GIT), urologic (UT), and hematologic toxicities (HT) of irradiation in patients treated with 3D-CRT with conventional fractionation, and VMAT either with hypo-fractionation for radical treatment, or with conventional fractionation for adjuvant treatment for PC, were evaluated. Materials and Methods: Fifty-seven consecutive patients with nonmetastatic prostate cancer treated at the Radiotherapy Section of Advanced Biomedical Sciences Department of the University "Federico II" (Naples, Italia) between January 2012 and May 2018 were retrospectively evaluated. Radiation treatment was delivered alone or after surgery with doses ranging between 66 and 76 Gy. Conventional fractionation was used in adjuvant setting (both 3DCRT and VMAT) and radical setting with 3D-CRT, while hypofractionation, $2.7 \mathrm{~Gy}$ fractions given 5 times per week with a total dose of $67.5 \mathrm{~Gy}$, was used only when VMAT technique was employed to irradiate radically the prostate. A high energy linear accelerator was used for treatment. Six static fields were used for 3D-CRT, while two coplanar dynamic arcs moving clockwise and counterclockwise were used for VMAT. The patients were checked weekly during the treatment and toxicity was recorded and graded according to Radiation Therapy Oncology Group (RTOG); blood test was taken before and during the treatment. Results: Patients median age was 71 years (range=47-82); median PSA level as measured before the beginning of radiation treatment was $3.6 \mathrm{ng} / \mathrm{ml}$. Thirty-six (63.1\%) patients had T-stage 2, $18(31.6 \%)$ had T-stage 3, while for $3(5.3 \%)$ patients this information was not available. Regarding the PC risk, 22 (38.6\%) patients resulted with intermediate risk disease, 17 (29.8\%) resulted with high risk disease, $16(28.1 \%)$ had a very high-risk disease; while for $2(3.5 \%)$ the information was not available. With a median follow-up of 34 months (range=6112), $4(7.0 \%)$ deaths were registered, two (3.5\%) of which directly related to the tumor. Twenty patients $(35.1 \%)$ had undergone surgery and twenty-seven patients (47.4\%) received hormone therapy in combination with RT. Acute GIT, UT and HT was observed in $42.1 \%, 80.7 \%$, and $24.6 \%$ of patients, respectively. With regard to fractionation scheme, significant differences in toxicity profile were not observed in the no surgery/no hormone therapy subgroup. Lymphopenia grade 1, grade 2 and grade 3 was observed in $1(1.7 \%), 2(3.5 \%)$ and $1(1.7 \%)$ patients, respectively. Grade 1 thrombocytopenia was found in $2(3.5 \%)$ patients; grade 1 anemia was observed in $10(17.5 \%)$ patients. No patients presented neutropenia. It was found late GIT in 2 (3.5\%) patients; $21(36.8 \%)$ presented irritative lower urinary tract symptoms. Conclusion: External beam radiation therapy is a favorable treatment option in nonmetastatic PC cancer. In our study, with the limit of a low number of patients, we found that different fractionation 
schedule (2.7 fraction/67.5 Gy vs. 2 Gy fraction/76 Gy) and treatment technique (VMAT vs. 3D-CRT) did not show statistically significant differences in acute GIT, UT, and HT. These results need to be validated with larger studies.

\section{0 \\ THE ROLE OF PREOPERATIVE CONTROLLING NUTRTIONAL STATUS (CONUT) SCORE IN THE ASSESSMENT OF PATHOLOGICAL FEATURES AND SURVIVAL OUTCOMES IN CLEAR-CELL RENAL CELL CARCINOMA (CCRCC): A POPULATION-BASED STUDY}

Matteo Boltri, Luca Ongaro, Francesco Claps, Francesca Migliozzi, Nicola Pavan, Michele Rizzo, Giovanni Liguori and Carlo Trombetta

Department of Urology, Azienda Sanitaria Universitaria Integrata di Trieste, Cattinara Hospital, Trieste, Italy Aim: To assess the correlation between pre-operative assessment of CONUT score and pathological features, overall survival (OS) and recurrence-free survival (RFS) in patients with primary diagnosis of clear-cell renal cell carcinoma (ccRCC) and no evidence of clinical nodal/distant metastatic disease before surgery. Materials and Methods: We retrospectively reviewed clinical data of patients treated with radical nephrectomy from 2006 to 2017. Lymph node dissection was performed for cases with intraoperative evidence of lymphadenopathy or at surgeons' discretion for patients deemed to be at high risk for occult nodal metastases at the time of surgery. A total of 110 patients was enrolled. For each patient CONUT score was determined considering the values of pre-operative albumin, total cholesterol and lymphocyte count. ROC curve was calculated and an optimal cut-off point was set at 1 . Patients were divided into 2 categories: low $(\leq 1)$ and high $(\geq)$ CONUT score. Univariate and multivariate analyses were performed. RFS and OS rates were calculated and compared between the two groups. Results: High-CONUT patients were more frequently males $(p=0.002)$ with pre-operative high mean fibrinogen level $(p=0.001)$, longer mean hospitalization $(p=0.012)$, even with no differences in Clavien-Dindo score $(p=0.32)$ and more severe pathological features ( $\mathrm{pT}$ stage $\geq 3, p=0.002$; $\mathrm{pN}+$, $p=0.005$; venous thrombus, $p=0.036$ ) than low-CONUT patients. No differences were observed in mean age and follow-up between the two cohorts. On multivariate analysis, high-CONUT was significantly associated with worse OS (HR=10.96, 95\% CI=2.31-52.15; $p=0.003)$, even if it did not result as an independent factor, comparing to age $(\mathrm{HR}=0.06$, $95 \% \mathrm{CI}=0.02-0.29 ; p<0.0001)$ and $\mathrm{pT}$ stage $\geq 3(\mathrm{HR}=2.63$, $95 \% \mathrm{CI}=1.41-4.91 ; \quad p=0.002)$. High-CONUT was not significantly associated with worse RFS (HR=1.41, $95 \% \mathrm{CI}=0.47-4.21 ; p=0.54)$. Conclusion: $\mathrm{CONUT}$ score is a low time-consuming and a cost-effective tool, which can be easily derived from blood values that are routinely checked before surgery. In this population-based study we found that in patients diagnosed with primary ccRCC with a clinical organ-confined disease, a pre-operative high-CONUT score was a strong predictor of worse pathological stage, lymphonode status and presence of renal vein thrombosis. Pre-operative evaluation of CONUT score could have clinical usefulness in patients with known histology (e.g. patients who underwent biopsy before surgery).

\section{2 \\ EFFECTS OF INTRAVESICAL INSTILLATIONS WITH HYALURONIC ACID ON IATROGENIC CYSTITIS}

Tommaso Di Vico ${ }^{1}$, Luca Brizzi ${ }^{1}$, Alessio Tognarelli ${ }^{1}$, Marco Lencioni ${ }^{1}$, Jacopo Durante ${ }^{1}$, Simone Malloggi ${ }^{1}$, Alessandro Perott ${ }^{\mathrm{i}}$, Lorenzo Tesi $^{1}$, Chiara Polito ${ }^{1}$, Iacopo Meneghetti ${ }^{1}$, Luca Mosillo ${ }^{1}$, Damiano Bracchitta ${ }^{1}$ and Filippo Maria Turri ${ }^{2}$

\author{
${ }^{1}$ Department of Translational Science and New \\ Technologies, Urology Unit - University \\ of Pisa, Pisa, Italy; \\ ${ }^{2}$ Department of Translational Science and \\ New Technologies, Urology Unit 2- AOUP, \\ University of Pisa, Pisa, Italy
}

Background/Aim: Iatrogenic cystitis is a common complication of pelvic radiotherapy for prostate cancer and intravesical chemo-immunotherapy for non-muscle invasive bladder cancer. Common signs and symptoms of this condition are pelvic pain, pollakiuria, urinary urgency, dysuria, haematuria and deterioration of quality of life. This clinical picture is due to the epithelial damage and is very similar to interstitial cystitis, which is the principal indication of hyaluronic acid instillations. The purpose of our study was to evaluate the impact of instillation therapy with hyaluronic acid on lower urinary tract symptoms (LUTS), quality of life and morphological urothelial aspects in patients who had developed cystitis after pelvic radiotherapy or intravesical chemo-immunotherapy with Bacillus Calmette-Guérine (BCG) or Mytomicin. Materials and Methods: We have evaluated 19 male patients undergone to bladder instillation therapy with hyaluronic acid for iatrogenic cystitis during the period 2012-2017. Of these, $12(67 \%)$ had developed cystitis after intravesical chemo-immunotherapy (7 with Mytomicin and 5 with BCG) and 7 (33\%) had developed cystitis after pelvic radiotherapy for prostate cancer. Mean number of instillations was 19.6 in post-BCG/Mytomicin cystitis group and 14.3 in post-radiotherapy cystitis group. Mean age of 
patients was 74.8 years in the first group and 68.7 years in the second group. We have investigated the intensity of urinary discomfort with a Visual Analogue Scale (VAS) and quality of life with I-PSS quality of life scale, before and after intravesical instillation. We have also performed cystoscopy before and after the treatment to estimate variations in bladder capacity and urothelial aspect. Results: The observed mean VAS improvement was 5.7 points (range=7.4-1.7) in the group of patients who had developed cystitis after intravesical immuno-chemotherapy, and 1.5 (range=3.5-2) in the group of patients undergone to pelvic radiotherapy. Quality of life improved in both groups (2.8 points and 1.3 respectively). Furthermore, we have qualitatively observed a significant reduction of visible hyperaemic and erythematous areas at cystoscopy. No side effects due to hyaluronic acid have been witnessed. Conclusion: Hyaluronic acid bladder instillations significantly reduce urinary discomfort in patients with iatrogenic cystitis, thus improving patients' quality of life. The impact of this treatment is more significant in postBCG/Mytomic cystitis than in post-radiotherapy cystitis. In patients with iatrogenic cystitis, acid hyaluronic instillations can be considered a reliable and safe therapy to reduce irritative symptoms and improve quality of life, especially after intravesical chemoimmunotherapy. The adoption of a score to objectively estimate variations in bladder capacity and urothelial aspects should be highly recommended.

\section{3}

\section{POLYPHENOL COMPOUNDS EXTRACTED FROM VACCINUM MYRTILLUS BILBERRY HAS CONCENTRATION-DEPENDENT ANTIPROLIFERATIVE EFFECTS ON HUMAN PROSTATE CANCER CELL LINES}

Riccardo Bartoletti $^{1}$, Claudia Di Serio ${ }^{2}$, Francesca Tarantini ${ }^{3}$, Andrea Ungar ${ }^{3}$, Tommaso Di Vico ${ }^{1}$, Jacopo Durante ${ }^{1}$, Alessio Tognarelli ${ }^{1}$,

Luca Brizzi ${ }^{1}$ and Massimo Del Bubba ${ }^{4}$

${ }^{1}$ Urology Unit, Department of Translational Research and New Technologies, University of Pisa, Pisa, Italy; ${ }^{2}$ Department of Medical and Surgical Critical Area, University of Florence, Florence, Italy;

${ }^{3}$ Geriatric Unit, Department of Medical and Surgical Critical Area, University of Florence, Florence, Italy; ${ }^{4}$ Department of Chemistry, University of Florence, Florence, Italy

Background/Aim: Recently it has been demonstrated that spontaneous bilberry Vaccinium myrtillus is an excellent source of dietary polyphenols. Polyphenolic compounds exhibit several properties such antioxidant, antimutagenic, antiinflammatory and anticancer activities. The anticancer activity of polyphenols is shown in a variety of human cancer cell lines both in vitro and in vivo, also in prostate cancer ( $\mathrm{PCa}$ ), one of the major medical problems in the male population. However, scientific evidences about polyphenols are still insufficient and wellconducted clinical studies are needed to clarify the efficacy of these molecules on PCa prevention and progression. The aim of this study was to investigate the effects of total polyphenols extracted from Vaccinium myrtillus on proliferation and apoptosis of three PCa cell lines with different degree of malignancy compared with normal epithelial prostate cells. Materials and Methods: The human PCa LNCaP, DU145, and PC3 cell lines and normal human prostate epithelial cells (PrEC) were used to perform proliferation experiment and apoptosis analysis. Cell growth was assessed in condition of anchorage dependency and independency (Anchorageindependent soft agar) adding increasing amounts of bilberry extract in culture medium. The Muse Annexin V \& Dead Cell Assay was performed utilizing Muse ${ }^{\mathrm{TM}}$ Cell Analyzer (Millipore) for apoptosis detection. Results: With increasing concentration of Vaccinium myrtillus extract, increasing inhibition of cell proliferation in all tumor cell lines was observed, with different degrees of potency between cell lines and having a low effect of inhibition on PrEC cells. In particular, higher concentrations of bilberry extract were necessary to obtain the same inhibitory effect on cell proliferation of androgen-independent DU145 and PC3 cells compared to androgen-dependent LNCap cells. Treatment of LNCaP cells growing in soft agar with Vaccinium myrtillus evidenced a reduction of growth capacity anchorage-dependent. Further, the bilberry extracts were evaluated for their ability to stimulate apoptosis. Flow cytometry analysis revealed that Vaccinium myrtillus extract increased the total number of apoptotic cells in all carcinoma cell lines. Conclusion: Our study provided data on the ability of bilberry extract to inhibit growth of PCa cells in a dose-dependent manner. Androgensensitive cell line LNCaP responded to treatment more than androgen insensitive DU145 and PC3, while normal epithelial prostate cells were resistant. Antiproliferative activity appeared to be mediated by pro-apoptotic mechanisms. These findings suggest Vaccinium myrtillus polyphenolic compounds as possible therapeutic agents against PCa. Other investigations should further focus on the bioavailable metabolites that are formed and their concentration in plasma and in prostate tissue.

\section{4}

\section{CORRELATION BETWEEN TUMOUR CHARACTERISTICS AND PERIOPERATIVE OUTCOMES IN NEPHRON SPARING SURGERY}

Simone Scarcella, Edoardo Agostini, Giulia Sbrollini, Carlo Giuliani, Giulio Milanese, Marco Tiroli, Lucio Dell'Atti and Andrea Benedetto Galosi 
Table I. Descriptive characteristics of the patient population at the time of renal tumour enucleation. All numbers are medians (interquartile range) and frequencies (proportions).

\begin{tabular}{|c|c|c|c|c|c|}
\hline Variables & $\begin{array}{l}\text { Overall population } \\
\quad(n=208 ; 100 \%)\end{array}$ & $\begin{array}{c}\text { Multiple masses } \\
\quad(\mathrm{n}=10 ; 5 \%)\end{array}$ & $\begin{array}{l}\text { Single renal unit } \\
\quad(\mathrm{n}=12 ; 6 \%)\end{array}$ & $\begin{array}{c}\text { Complex lesions } \\
(\mathrm{n}=92 ; 44 \%)\end{array}$ & $p$-Value \\
\hline Age at RP (years) & $67(57,74)$ & $64(54,69)$ & $72(58,78)$ & $67(55,75)$ & 0.7 \\
\hline BMI & $26(23,29)$ & $28(25,33)$ & $26(22,27)$ & $26(24,29)$ & 0.2 \\
\hline \multicolumn{6}{|l|}{ Gender } \\
\hline Male & $107(51 \%)$ & $6(60 \%)$ & $8(66.7 \%)$ & $49(53.3 \%)$ & \multirow[t]{2}{*}{0.4} \\
\hline Female & $101(49 \%)$ & $4(40 \%)$ & $4(33.3 \%)$ & $43(46.7 \%)$ & \\
\hline \multicolumn{6}{|l|}{ Side } \\
\hline Dx & $122(59 \%)$ & $6(60 \%)$ & $6(50 \%)$ & $50(54.3 \%)$ & \multirow[t]{2}{*}{0.5} \\
\hline $\mathrm{Sx}$ & $86(41 \%)$ & $4(40 \%)$ & $6(50 \%)$ & $42(45.7 \%)$ & \\
\hline \multicolumn{6}{|l|}{ ASA } \\
\hline I & $40(19 \%)$ & $2(20 \%)$ & $3(25 \%)$ & $17(18.5 \%)$ & \multirow[t]{3}{*}{0.4} \\
\hline II & $135(65 \%)$ & $4(40 \%)$ & $8(66.7 \%)$ & $58(63.0 \%)$ & \\
\hline III & $33(16 \%)$ & $4(40 \%)$ & $1(8.3 \%)$ & $17(18.5 \%)$ & \\
\hline Pre-Op GFR, $\mathrm{ml} / \mathrm{min} / 1,73 \mathrm{~m}^{2}$ & $86(67,102)$ & $73(52,89)$ & $51.4(44,73)$ & $82(61,101)$ & 0.01 \\
\hline \multicolumn{6}{|l|}{ CKD grade Pre-OP } \\
\hline I & $96(46.1 \%)$ & $2(20 \%)$ & $2(16.7 \%)$ & $41(44.6 \%)$ & \multirow[t]{5}{*}{0.001} \\
\hline II & $74(35.5 \%)$ & $5(50 \%)$ & $2(16.7 \%)$ & $29(31.5 \%)$ & \\
\hline III & $34(16.3 \%)$ & $20(20 \%)$ & $8(66.7 \%)$ & $20(21.7 \%)$ & \\
\hline IV & $2(1 \%)$ & $1(10 \%)$ & l & $1(1.1 \%)$ & \\
\hline $\mathrm{V}$ & $2(1 \%)$ & 1 & 1 & $1(1.1 \%)$ & \\
\hline Renal score & $7(6,8)$ & $6(4,7)$ & $8(6,10)$ & $9(8,11)$ & 0.02 \\
\hline
\end{tabular}

BMI, Body mass index; ASA, American Society of Anesthesiologists Classification; GFR, glomerular filtration rate; CKD, chronic kidney disease.

Division of Urology, Ancona University Hospital, Bologna, Italy

Background/Aim: Partial nephrectomy (PN) is considered the reference treatment for patients with $\mathrm{T} 1 \mathrm{a}$ renal cell carcinoma (RCC) because it provides equivalent oncological, functional, and overall survival outcomes compared to radical nephrectomy ( $\mathrm{RN})$. Furthermore, it is associated with lower risk of developing chronic kidney disease (CKD) and long-term cardiovascular diseases, even if it is a more technically complex procedure with higher rates of complications (1). With these principles in mind, and according to EAU guidelines the majority of patients with clinical stage I RCC is surgically treated with PN in order to preserve the largest amount of the healthy renal parenchyma without compromising the oncologic radicality. On the other hand, the use of such a conservative approach in patients with large or anatomically complex masses could potentially increase the oncological risk in comparison to a more radical technique (2). The aim of our study was to evaluate the correlation between characteristics of renal masses and functional/oncological outcomes in a cohort of patients treated with nephron sparing surgery (NSS). Patients and Methods: We evaluated 208 patients admitted for the treatment of kidney parenchymal cancer. All patients underwent NSS with either a laparoscopic or an open approach. Among these, 92 (44\%) patients were submitted to conservative surgery for anatomically complex renal masses defined as: $10(5 \%)$ patients with multifocal lesions, $12(6 \%)$ with solid tumours in solitary kidney, and $70(34 \%)$ for lesions $>4 \mathrm{~cm}$ and a renal nephrometry score $>9$ points. Baseline tumour stage was: 38 (41.3\%) cT1a, 43 (46.7\%) cT1b, $11(12 \%)$ cT2. Median RENAL score was: 6 (IQR=47) for the multiple masses (MM) group, 8 (IQR=6-10) for the single renal unit (SU) group and $9(\mathrm{IQR}=8-11)$ for the complex masses (CM) group. Pre-operative renal function impairment was staged according to estimated glomerular filtration rate (eGFR): median (IQR) values were lower in the single kidney unit $51.4(\mathrm{IQR}=44-73)$ and in the multiple renal masses groups 73 (IQR=52-89) compared to the the complex masses group $82(\mathrm{IQR}=61-101)(p=0.01)$. Chronic renal failure (CRF) was defined as an eGFR value lower than $60 \mathrm{ml} / \mathrm{min} / 1.73 \mathrm{~m}^{2}$ and present in $22(23.9 \%)$ patients with complex renal masses (Table I). In descriptive analysis, all continuous variables were summarized with median and IQR, while categorical variables with absolute and relative frequencies. Clinical and post-operative variables were compared using logistic regression, T-test and Chi squared test. Statistical analyses have been performed with IBM SPSS Statistics v.20. Results: All procedures were performed 
Table II. Descriptive characteristics of the patient population after renal tumour enucleation. All numbers are medians (interquartile range) and frequencies (proportions).

\begin{tabular}{|c|c|c|c|c|c|}
\hline Variables & $\begin{array}{l}\text { Overall population } \\
(\mathrm{n}=208 ; 100 \%)\end{array}$ & $\begin{array}{c}\text { Multiple masses } \\
(\mathrm{n}=10 ; 5 \%)\end{array}$ & $\begin{array}{l}\text { Single renal unit } \\
(\mathrm{n}=12 ; 6 \%)\end{array}$ & $\begin{array}{l}\text { Complexlesions } \\
(\mathrm{n}=92 ; 44 \%)\end{array}$ & $p$-Value \\
\hline Post-op GFR, $\mathrm{ml} / \mathrm{min} / 1,73 \mathrm{~m}^{2}$ & $80(62,99)$ & $68(46,89)$ & $41.5(33,55)$ & $78(56,96)$ & 0.002 \\
\hline \multicolumn{6}{|l|}{ CKD grade Post-OP } \\
\hline I & $77(37 \%)$ & $2(20 \%)$ & $1(8.3 \%)$ & $33(35.9 \%)$ & \multirow[t]{5}{*}{0.001} \\
\hline II & $83(39.9 \%)$ & $4(40 \%)$ & 1 & $35(38 \%)$ & \\
\hline III & $35(16.8 \%)$ & $3(30 \%)$ & $10(83.3 \%)$ & $18(19.6 \%)$ & \\
\hline IV & $7(3.4 \%)$ & $1(10 \%)$ & $1(8.3 \%)$ & $5(5.4 \%)$ & \\
\hline $\mathrm{V}$ & $2(1 \%)$ & 1 & 1 & $1(1.1 \%)$ & \\
\hline \multicolumn{6}{|l|}{ CKD impairment } \\
\hline Yes & $42(20.2 \%)$ & $2(20 \%)$ & $4(33.3 \%)$ & $20(21.8 \%)$ & \multirow[t]{2}{*}{0.9} \\
\hline No & $166(79.8 \%)$ & $8(80 \%)$ & $8(66.7 \%)$ & $72(78.2 \%)$ & \\
\hline $\mathrm{EBL}, \mathrm{ml}$ & $200(100,328)$ & $265(188,375)$ & $200(100,300)$ & $250(103,398)$ & 0.5 \\
\hline \multicolumn{6}{|l|}{ Ischemia } \\
\hline Yes & $69(33.2 \%)$ & $5(50 \%)$ & $1(8.3 \%)$ & $38(41.3 \%)$ & \multirow[t]{2}{*}{0.02} \\
\hline No & $139(66.8 \%)$ & $5(50 \%)$ & $11(91.7 \%)$ & $54(58.7 \%)$ & \\
\hline WIT, min & $8(0,14)$ & $8(4,12)$ & $5(0, \mathrm{NA})$ & $9(0,14)$ & 0.4 \\
\hline $\mathrm{OT}, \mathrm{min}$ & $125(95,150)$ & $150(139,225)$ & $176(125,208)$ & $130(103,165)$ & 0.02 \\
\hline \multicolumn{6}{|l|}{ Pathologic $\mathrm{T}$ stage } \\
\hline pT1a & $133(64.0 \%)$ & $8(80 \%)$ & $10(83.3 \%)$ & $17(18.5 \%)$ & \multirow[t]{5}{*}{0.001} \\
\hline pT1b & $59(28.3 \%)$ & $2(20 \%)$ & $1(8.3 \%)$ & $59(64.1 \%)$ & \\
\hline pT2a & $10(4.8 \%)$ & 1 & $1(8.3 \%)$ & $10(10.9 \%)$ & \\
\hline pT2b & $2(1.0 \%)$ & l & 1 & $2(2.2 \%)$ & \\
\hline pT3a & $4(1.9 \%)$ & l & l & $4(4.3 \%)$ & \\
\hline \multicolumn{5}{|l|}{ Surgical margins } & \multirow[t]{3}{*}{0.8} \\
\hline Negative & $188(90.4 \%)$ & $10(100 \%)$ & $11(91.7 \%)$ & $85(92.4 \%)$ & \\
\hline Positive & $20(9.6 \%)$ & $0(0 \%)$ & $1(8.3 \%)$ & $7(7.6 \%)$ & \\
\hline \multicolumn{5}{|l|}{ MIC score } & \multirow[t]{3}{*}{0.5} \\
\hline Yes & $156(75.5 \%)$ & $9(90 \%)$ & $11(91.7 \%)$ & $73(79.3 \%)$ & \\
\hline No & $51(24.5 \%)$ & $1(10 \%)$ & $1(8.3 \%)$ & $19(20.7 \%)$ & \\
\hline LOS, days & $5(4,6)$ & $5(4,7)$ & $5(4,7)$ & $5(4,7)$ & 0.2 \\
\hline \multicolumn{6}{|l|}{ Post-Op Clavien Dindo } \\
\hline No & $174(83.7 \%)$ & $8(80 \%)$ & $11(91.7 \%)$ & $75(81.5 \%)$ & \multirow[t]{5}{*}{0.7} \\
\hline I & $14(6.7 \%)$ & $1(10 \%)$ & $1(8.3 \%)$ & $4(4.3 \%)$ & \\
\hline II & $12(5.8 \%)$ & $1(10 \%)$ & 1 & $9(9.8 \%)$ & \\
\hline III & $4(1.9 \%)$ & 1 & I & $2(2.2 \%)$ & \\
\hline IV & $4(1.9 \%)$ & I & I & $2(2.2 \%)$ & \\
\hline
\end{tabular}

GFR, Glomerular filtration rate; CKD, chronic kidney disease; EBL, estimated blood loss; WIT, warm ischemia time; OT, operative time; MIC, margin, ischemia and complication score.

with open technique and retroperitoneal approach, using artery clamping as needed during the procedure in 38 $(41.3 \%)$ patients in the complex masses group, in $1(8.3 \%)$ patients in the single renal unit group, in $5(50 \%)$ patients of the multiple renal masses group $(p=0.02)$. Median warm ischemia time was $8 \mathrm{~min}(\mathrm{IQR}=4-12)$ in the $\mathrm{MM}, 5 \mathrm{~min}$ (IQR=0-NA) in the $\mathrm{SU}$, and $9 \mathrm{~min}(\mathrm{IQR}=0-14)$ in the $\mathrm{CM}$ group ( $p=0.4)$; when ischemia was not necessary, a clampless procedure was performed with controlled hypotension. Median estimated blood loss was in the MM group $265 \mathrm{ml}$ $(\mathrm{IQR}=188-375), 200 \mathrm{ml}(\mathrm{IQR}=100-300)$ in the SU, $250 \mathrm{ml}$ $(\mathrm{IQR}=103-398)$ in the CM $(p=0.5)$ and post-operative blood transfusion were necessary in 1 patient $(10 \%), 2$ patients
$(16.7 \%)$ and $15(15.3 \%)(p=0.02)$, respectively. Median operative time was $130 \mathrm{~min}(\mathrm{IQR}=103-165)$ in the $\mathrm{CM}$ group and the analysis of the MM and SU subgroups revealed a higher time, $150 \mathrm{~min}(\mathrm{IQR}=139=225)$ and $176 \mathrm{~min}$ (IQR=125-208), respectively $(p=0.02)$. Margin Ischemia and Complication (MIC) score was achieved in 9 patients $(90 \%)$ of the MM group, 11 patients $(91.7 \%)$ of the SU and 73 (79.3\%) of the CM (Table II) $(p=0.5)$. Conversion to radical nephrectomy was necessary in 4 patients $(4.3 \%)$ of the CM group. Intra-operative complications are reported in greater detail in Table III. Discussion and Conclusion: Patients undergoing NSS for complex masses, multifocal masses or in single renal unit could be exposed to higher rates of 
Table III. Descriptive characteristics of the patient population after renal tumour enucleation. All numbers are medians (interquartile range) and frequencies (proportions).

\begin{tabular}{|c|c|c|c|c|c|}
\hline Variables & $\begin{array}{l}\text { Overall population } \\
(\mathrm{n}=208 ; 100 \%)\end{array}$ & $\begin{array}{l}\text { Multiple masses } \\
\quad(\mathrm{n}=10 ; 5 \%)\end{array}$ & $\begin{array}{l}\text { Single renal unit } \\
\quad(\mathrm{n}=12 ; 6 \%)\end{array}$ & $\begin{array}{l}\text { Complex lesions } \\
(\mathrm{n}=92 ; 44 \%)\end{array}$ & $p$-Value \\
\hline \multicolumn{6}{|l|}{$\begin{array}{l}\text { Perioperative blood } \\
\text { transfusion, units }\end{array}$} \\
\hline No & $184(88.5 \%)$ & $9(90 \%)$ & $10(83.3 \%)$ & $77(83.7 \%)$ & 0.02 \\
\hline I & $17(8.2 \%)$ & I & $2(16.7 \%)$ & $12(13.0 \%)$ & \\
\hline II & $5(2.4 \%)$ & I & 1 & $2(2.2 \%)$ & \\
\hline III & $1(0.5 \%)$ & I & I & l & \\
\hline IV & $1(0.5 \%)$ & $1(10 \%)$ & I & $1(1.1 \%)$ & \\
\hline \multicolumn{6}{|c|}{ Intra-Op Complications } \\
\hline No & $191(91.8 \%)$ & $9(90 \%)$ & $7(58.3 \%)$ & $84(91.3 \%)$ & 0.001 \\
\hline Urinary tract lesion & $6(2.9 \%)$ & / & $4(33.3 \%)$ & $4(4.3 \%)$ & \\
\hline Pleural lesion & $3(1.4 \%)$ & / & $1(8.3 \%)$ & $1(1.1 \%)$ & \\
\hline Intestinal lesion & $1(0.5 \%)$ & I & I & l & \\
\hline Liver lesion & $1(0.5 \%)$ & / & / & I & \\
\hline Spleen lesion & $6(2.9 \%)$ & $1(10 \%)$ & I & $3(3.3 \%)$ & \\
\hline \multicolumn{6}{|l|}{ Haemostatic agents } \\
\hline No & $89(42.8 \%)$ & $4(40 \%)$ & $9(75 \%)$ & $37(40.2 \%)$ & 0.3 \\
\hline Floseal & $84(40.4 \%)$ & $6(60 \%)$ & $3(25 \%)$ & $39(42.4 \%)$ & \\
\hline Surgiflow & $6(2.9 \%)$ & 1 & 1 & $2(2.2 \%)$ & \\
\hline Tachosil & $29(13.9 \%)$ & / & / & $14(15.2 \%)$ & \\
\hline \multicolumn{6}{|l|}{ Uretheral catheter } \\
\hline Yes & $34(16.3 \%)$ & $2(20 \%)$ & $3(25 \%)$ & $17(18.5 \%)$ & 0.8 \\
\hline No & $174(83.7 \%)$ & $8(80 \%)$ & $9(75 \%)$ & $75(81.5 \%)$ & \\
\hline \multicolumn{6}{|l|}{ Conversion RN } \\
\hline Yes & $4(1,9 \%)$ & $0(0 \%)$ & $0(0 \%)$ & $4(4.3 \%)$ & 0.001 \\
\hline No & $204(98.1 \%)$ & $10(100 \%)$ & $12(100 \%)$ & $88(95.7 \%)$ & \\
\hline
\end{tabular}

RN, Radical nephrectomy.

perioperative complications and oncological risks that could be avoided by a radical approach. Nevertheless, in patients with chronic renal function impairment is mandatory to maximize the preservation of healthy renal parenchyma to lower the risk of renal unit loss. The MIC score is a tool that can be used to identify those patients who achieved an optimal outcome after PN. In order to meet the MIC criteria, surgical margins must be negative, warm ischemia time must be lower than $20 \mathrm{~min}$ and no major complications (grade 34 according to Clavien classification) must be observed (3). NSS was technically feasible in $88 / 92(95.7 \%)$ patients with anatomically complex renal masses and MIC score was achieved in $73 / 92(79 \%)$ of surgeries, with no significant difference noted among the subgroups. Vascular clamping was avoided in 54/92 (58.7\%) of cases, especially in patients with preoperative renal impairment. When necessary, the median WIT was limited to 9 min (0-14) using delayed clamping and early unclamping, with restricted blood losses. The need for transfusions was significantly higher in the complex masses group when compared to the non-complex one. The median operative time was significantly longer in the multiple masses and single unit subgroups than in the complex masses group. These findings confirm that, in our experience, stratifying patients selected for PN according to tumour's characteristics allows a better prediction of the surgical outcome.

1 Kim SP, Thompson RH, Boorjian SA, Weight CJ, Han LC, Murad MH, Shippee ND, Erwin PJ, Costello BA, Chow GK, Leibovich BC: Comparative effective- ness for survival and renal function of partial and radical nephrectomy for localized renal tumors: a systematic review and meta-analysis. J Urol 188: 51-57, 2012. PMID: 22591957. DOI: 10.1016/j.juro.2012.03.006

2 Joniau S, Vander Eeckt K, Srirangam SJ and Van Poppel $\mathrm{H}$ : Outcome of nephron-sparing surgery for $\mathrm{T} 1 \mathrm{~b}$ renal cell carcinoma. BJU Int 103: 1344-1348, 2009. PMID: 19040528. DOI: 10.1111/j.1464-410X.2008.08230.x

3 Buffi N, Lista G, Larcher A, Lughezzani G, Ficarra V, Cestari A, Lazzeri M and Guazzoni G: Margin, ischemia, and complications (MIC) score in partial nephrectomy: A new system for evaluating achievement of optimal outcomes in nephron-sparing surgery. Eur Urol 62: 617-618, 2012. PMID: 22704367. DOI: 10.1016/j.eururo. 2012.06.001 
125

\section{USE OF RENAL SCORING SYSTEMS IN NEPHRON SPARING SURGERY: APPLICABILITY AND REPRODUCIBILITY FROM RESIDENTS PERSPECTIVE}

$\underline{\text { Simone Scarcella }}^{1}$, Edoardo Agostini $^{1}$, Giulia Sbrollini ${ }^{1}$, Andrea Rochira ${ }^{2}$, Lucio Dell'Atti ${ }^{1}$ and Andrea Benedetto Galosi ${ }^{1}$

${ }^{1}$ Division of Urology, Ancona University

Hospital, Bologna, Italy;

${ }^{2}$ Department of Biomedicine and Prevention, Alma Mater Studiorum Bologna, Bologna, Italy

Background/Aim: The incidence of small renal masses has steadily increased in the last decade (1). Advances both in imaging and surgical techniques led to a shift from radical treatment to a conservative one with expanding indications for nephron sparing surgery (NSS). Furthermore, the use of minimally invasive techniques, such as robotassisted/laparoscopic partial nephrectomy, cryoablation, and experimental trial of active surveillance, are widely accepted (2). In this setting preoperative imaging studies are of paramount importance in treatment decision making and in assisting physicians to determine the best surgical approach. Several scoring systems (SS) have been developed to stratify patients and to help in predicting surgical complexity and potential post-surgical outcomes in NSS (3). However, there is not a reference standard and the variety of different SS, which are not interchangeable one to another, thus it could be misleading when reporting preoperative assessment between different institutions. To date, it is not known how these SS vary between readers with different backgrounds and levels of expertise. The aim of this study was to evaluate the applicability of four different nephrometry SSs in a reallife scenario, and to analyze their reproducibility by comparing the scores assigned by radiology and urology residents. Patients and Methods: We enrolled 36 patients with a suspected diagnosis of renal tumor. All patients were candidate to NSS with a laparoscopic or open approach between November 2017 and June 2018. All diagnostic computed tomography (CT) scans were retrospectively evaluated and images were consulted both in the axial and coronal planes. Six residents in total, 3 urologists and 3 radiologists, respectively at the first, second and last year of residency analyzed diagnostic CT scans and assigned independently the nephrometry scores for each patient. We included the RENAL (radius, exophytic/endophytic properties, nearness of tumor to the collecting system or sinus in millimeters, anterior/posterior location relative to polar lines), the PADUA (preoperative aspects and dimensions used for anatomic classification), the C-Index (centrality-index) and the ABC (arterial based complexity) SSs. In descriptive analysis, all continuous variables were summarized with median and interquartile range, while categorical variables with absolute and relative frequencies. The scores given by the "Urologists group" and the "Radiologists group" were compared using the MannWhitney test and graphically with the Box Plot. Intra-class correlation coefficient (ICC) was used for continuous scores reliability, while for categorical scores Cohen's kappa $(\mathrm{k})$ was used. Inter-observer reliability was calculated, first comparing the scores among all readers, then among subgroups of urologists and radiologists only, and finally

Table I. Intraclass correlation and Cohen's Kappa [95\% confidence interval (CI)] amongst readers.

\begin{tabular}{lcccc}
\hline & $\begin{array}{c}\text { RENAL } \\
\text { ICC }(95 \% \mathrm{CI})\end{array}$ & $\begin{array}{c}\text { PADUA } \\
\text { ICC (95\%CI) }\end{array}$ & $\begin{array}{c}\text { C-Index } \\
\text { ICC (95\%CI) }\end{array}$ & Kappa (95\%CI) \\
\hline All readers & $0.80(0.45-0.78)$ & $0.82(0.74-0.90)$ & $0.40(0.24-0.55)$ & $0.52(0.42-0.62)$ \\
Urologists only & $0.91(0.86-0.96)$ & $0.86(0.78-0.93)$ & $0.81(0.71-0.90)$ & $0.81(0.71-0.91)$ \\
Radiologists only & $0.80(0.70-0.90)$ & $0.83(0.74-0.91)$ & $0.46(0.26-0.66)$ & $0.32(0.21-0.43)$ \\
\hline
\end{tabular}

ICC, Intra-class correlation coefficient.

Table II. Median and interquartile range (IQR): Comparison between urologists and radiologists.

\begin{tabular}{lcccc}
\hline & RENAL & PADUA & C-Index & ABC \\
Median (IQR) & Median (IQR) & Median (IQR) & $1.8(1.3-2.6)$ & $2(2-3 \mathrm{~s})$ \\
Urologists & $8(7-9)$ & $8(7-10)$ & $2.4(1.8-3.3)$ & $2(2-3 \mathrm{~s})$ \\
Radiologists & $7(6-9)$ & $8(7-10)$ & $<0.001$ & 0.612 \\
$p$-Value (Mann-Withney test) & 0.012 & 0.560 & & \\
\hline
\end{tabular}


between different specialist groups. Moreover, we compared the mean differences in scores assigned by the two groups of urologists and radiologists. Statistical analyses have been performed with IBM SPSS Statistics 20. Results: ICC among all readers was higher for the RENAL and PADUA scores while was low for the C-Index and ABC score. When considering the specific professional background, all urologist SS where characterized by an intra-class correlation index higher than 0.8 , while in the radiologist group only the RENAL and the PADUA presented similar results. On the contrary the C-Index and ABC scores had lower ICC (Table I). The differences in scores assigned between the two groups of specialists resulted respectively in 1 point and 0.6 point mean for the RENAL score $(p=0.012)$ and the C-Index $(p<0.001)$ while no mean differences were observed in PADUA and ABC scores $(p>0.05)$ (Table II). Discussion and Conclusion: Preoperative planning in patients with renal cortical neoplasms relies on anatomical tumor characteristics and the use of SS allows including important variables, such as tumor size and locations, in a standardized manner. The use of these SS should not be complex in its application and has to be based on objective measurements in order to represent the technical complexity of the planned surgery. All scores represent a standardized method for quantitatively reporting and categorizing renal tumors and can guide clinicians in choosing among different surgical approaches. Our results showed that SS based on anatomical characteristics are more reproducible: PADUA and RENAL score had the higher reproducibility among all groups. Moreover, in the urologist group only there was a significantly higher reproducibility of all the evaluated scores. This finding could be related to the daily-practice use of these SSs by the urologist group compared to the radiologist group. On the other hand, C-Index score tended to differ significantly between urologists and radiologists, even inside the same specialty. Our study presents some limitations; first, the size of the cohort was relatively small and larger number of patients is needed to verify our results. Second, all scores were assigned by residents and these results should be further compared with a reference set of scores given by Consultant Urologist or Radiologist. Moreover, our work focused only on the assessment of nephrometry score and did not explore the correlation between different scores and peri-postoperative outcomes which is fundamental for their clinical and surgical utility. Despite these limits, our results suggest that SSs based on anatomical characteristics tend to show a higher reproducibility even among residents with different professional backgrounds. Finally, considering that RENAL and PADUA scores showed the best accordance between groups, these SSs should be adopted and incorporate also in the preoperative imaging report from radiologists.

1 Bray F, Ferlay J, Soerjomataram I, Siegel RL, Torre LA and Jemal A: Global cancer statistics 2018: GLOBOCAN estimates of incidence and mortality worldwide for 36 cancers in 185 countries. CA Cancer J Clin 68(6): 394-424, 2018. PMID: 30207593. DOI: $10.3322 /$ caac. 21492

2 Hora M, Eret V, Trávníček I, Procházková K, Pitra T, Dolejšová $\mathrm{O}$, Hes $\mathrm{O}$ and Petersson F: Surgical treatment of kidney tumors - Contemporary trends in clinical practice. Cent Eur J Urol 69(4): 341-346, 2016. PMID: 28127448. DOI: $10.5173 /$ ceju.2016.845

3 Okhunov Z, Rais-Bahrami S, George AK, Waingankar N, Duty B, Montag S, Rosen L, Sunday S, Vira MA and Kavoussi LR: The comparison of three renal tumor scoring systems: C-Index, P.A.D.U.A., and R.E.N.A.L. Nephrometry Scores. J Endourol 25(12): 1921-1924, 2011. PMID: 21905850. DOI: $10.1089 / \mathrm{end} .2011 .0301$

\section{6}

\section{URETHRAL SUSPENSION DURING OPEN RETROPUBIC RADICAL PROSTATECTOMY: A NOVEL METHOD TO IMPROVE EARLY POSTOPERATIVE RECOVERY OF URINARY CONTINENCE}

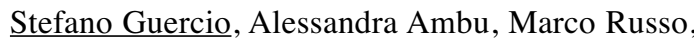 \\ Mariateresa Carchedi, Antonino Battaglia, \\ Giulio Bonvissuto and Maurizio Bellina \\ Department of Urology, Ospedale degli Infermi \\ di Rivoli, Turin, Italy
}

Background/Aim: Postoperative urinary incontinence after radical prostatectomy (RP) may greatly affect patients' quality of life and may require a long time and further treatments to be addressed (1). We show our results with a novel technique for urethral suspension during RP, which is a modification of the technique originally described by JW Thuroff et al. (2); this latter considered the use of a single sling, harvested from the rectus muscle fascia, while we created one fascial limb on each side of the linea alba, then suturing both limbs to the vesico-urethral anastomosis, in order to suspend it and to avoid its downward dislocation. Materials and Methods: From June 2017 to October 2017, 40 patients with localized prostate cancer underwent to open $\mathrm{RP}$ at our institution, with nerve-, bladder neck sparing technique. Our standard technique for the anastomosis includes an interrupted suture with 3 stitches on both sides (towards 11, 9, 7 on the left, and toward 1,3,5 on the right), and a running suture on the posterior urethral plate. For the modified technique, once the anastomosis is sutured, 2 limbs of rectus muscle fascia on both sides of the linea alba are prepared, each $8 \mathrm{~cm}$ long and $1 \mathrm{~cm}$ wide, with a distal attachment; the free extremity is brought to the anastomosis and sutured to the stitch towards 7 on the left and to the stitch towards 5 on the right, under a mild perineal pressure 
to enhance the urethral suspension. In 20 patients (group 1) the standard vesico-urethral anastomosis was performed without additional procedures, while in 20 patients (group 2) a suspension of the anastomosis was added to the standard vesico-urethral anastomosis. Continence results were evaluated in terms of number of pads per day and according to the International Consultation on Incontinence Questionnaire (ICIQ) score at 24 and $48 \mathrm{~h}$ and at 4 weeks postoperatively. Continence was defined as the need of 0-1 pad per day. Results: Continence rate (CR) for group 1 and group 2 was $15 \%$ vs. $40 \%$ at $24 \mathrm{~h} 20 \%$ vs. $50 \%$ at $48 \mathrm{~h}$, and $30 \%$ vs. $70 \%$ at 4 weeks, respectively. No urinary obstructive complications were recorded in patients who received a modified anastomosis suspension technique. Conclusion: Although on a small series of patients, our results show better early continence results for the patients who received a urethral suspension according to our technique, compared to patients who underwent RP according to a standard anastomosis technique without vesico-urethral anastomosis suspension. The mechanism on which early recovery of urinary continece following urethral suspension may be related, is basically unknown. Our hypothesis is that vesicourethral anastomosis suspension created with 2 limbs of rectus muscle fascia may provide an additional support to the urethral striated sphincter, and a further stabilisation of posterior urethra within the pelvic diaphragm, while avoiding the potential obstructive complications on urinary flow related to sling compression.

1 Nelson JB: The ongoing challenge of urinary incontinence after radical prostatectomy. J Urol 198: 1223-1225, 2017. PMID: 28919315. DOI: 10.1016/j.juro.2017.09.053

2 Fokaefs ED, Lampel A, Hohenfellner M, Lazica M and Thuroff JW: Experimental evaluation of free versus pedicled fascial flaps for sling surgery of urinary stress incontinence. J Urol 157: 1039-1043, 1997. PMID: 9072539. 


\section{Authors Index*}

(Figures indicate abstract number. *Missing abstracts were withdrawn.)

Aguiari G, 12

Alongi F, 13, 14

Badenchini F, 91, 111

Baiamonte D, 16

Bartoletti R, 123

Bertolo R, 40

Bianchi L, 44, 49

Boltri M, 120

Bortolato B, 3

Capretti C, 52

Caputo A, 84

Catanzaro MA, 115

Cazzaniga W, 41

Claps F, 77, 98, 99

Corrao G, 37

Cuccia F, 80, 81

D'elia C, 35, 38

De Luca L, 92

De Luca S, 24, 58

De Robbio J, 119

De Zorzi L, 21

Dell'Atti L, 10

Di Florio T, 94

Di Vico T, 122

Dordoni P, 83

Ennas M, 18

Falavolti C, 8

Ferella L, 97

Fionda B, 76

Fiorello N, 5, 15

Franceschini T, 118

Franzese C, 67

Frassinelli L, 22

Grimaldi G, 106

Guercio S, 126
La Croce G, 45, 54, 56, 57

Lorenzo Bonù M, 43

Luzzago S, 101

Magli A, 19

Maiolino G, 20, 63

Manfredi M, 48

Mantello G, 103, 104

Marra G, 65, 105, 113, 114

Mistretta FA, 107, 108, 110

Montesi L, 62

Napodano G, 30, 31

Nicolai N, 112

Oderda M, 69

Olivieri V, 1, 2, 4, 86

Pastore F, 85

Porpiglia F, 73

Prayer Galetti T, 70, 72

Rancati T, 75

Rese A, 93

Rigo M, 27

Riva G, 23

Roscigno M, 50

Sanseverino R, 33

Scalici Gesolfo, C, 9

Scarcella S, 124, 125

Schiavina R, 53

Serretta V, 28, 39

Silecchia G, 7

Soatti CP, 116, 117

Soligo M, 47, 55, 64, 66

Sorce C, 96

Soria F, 87, 88, 89, 90

Trenti E, 71

Volpe S, 36

Vukcaj S, 17 